\title{
High Mountain Areas
}

\author{
Coordinating Lead Authors: \\ Regine Hock (USA), Golam Rasul (Nepal)
}

\section{Lead Authors:}

Carolina Adler (Switzerland/Australia), Bolívar Cáceres (Ecuador), Stephan Gruber (Canada/ Germany), Yukiko Hirabayashi (Japan), Miriam Jackson (Norway), Andreas Kääb (Norway), Shichang Kang (China), Stanislav Kutuzov (Russian Federation), Alexander Milner (UK), Ulf Molau (Sweden), Samuel Morin (France), Ben Orlove (USA), Heidi Steltzer (USA)

\section{Contributing Authors:}

Simon Allen (Switzerland), Lukas Arenson (Canada), Soumyadeep Banerjee (India), lestyn Barr (UK), Roxana Bórquez (Chile), Lee Brown (UK), Bin Cao (China), Mark Carey (USA), Graham Cogley (Canada), Andreas Fischlin (Switzerland), Alex de Sherbinin (USA), Nicolas Eckert (France), Marten Geertsema (Canada), Marca Hagenstad (USA), Martin Honsberg (Germany), Eran Hood (USA), Matthias Huss (Switzerland), Elizabeth Jimenez Zamora (Bolivia), Sven Kotlarski (Switzerland), Pierre-Marie Lefeuvre (Norway/France), Juan Ignacio López Moreno (Spain), Jessica Lundquist (USA), Graham McDowell (Canada), Scott Mills (USA), Cuicui Mou (China), Santosh Nepal (Nepal), Jeannette Noetzli (Switzerland), Elisa Palazzi (Italy), Nick Pepin (UK), Christian Rixen (Switzerland), Maria Shahgedanova (UK), S. McKenzie Skiles (USA), Christian Vincent (France), Daniel Viviroli (Switzerland), Gesa Weyhenmeyer (Sweden), Pasang Yangjee Sherpa (Nepal/USA), Nora M. Weyer (Germany), Bert Wouters (Netherlands), Teppei J. Yasunari (Japan), Qinglong You (China), Yangjiang Zhang (China)

\section{Review Editors:}

Georg Kaser (Austria), Aditi Mukherji (Nepal/India)

\section{Chapter Scientist:}

Pierre-Marie Lefeuvre (Norway/France), Santosh Nepal (Nepal)

This chapter should be cited as:

Hock, R., G. Rasul, C. Adler, B. Cáceres, S. Gruber, Y. Hirabayashi, M. Jackson, A. Kääb, S. Kang, S. Kutuzov, Al. Milner, U. Molau, S. Morin, B. Orlove, and H. Steltzer, 2019: High Mountain Areas. In: IPCC Special Report on the Ocean and Cryosphere in a Changing Climate [H.-O. Pörtner, D.C. Roberts, V. Masson-Delmotte, P. Zhai, M. Tignor, E. Poloczanska, K. Mintenbeck, A. Alegría, M. Nicolai, A. Okem, J. Petzold, B. Rama, N.M. Weyer (eds.)]. Cambridge University Press, Cambridge, UK and New York, NY, USA, pp. 131-202. https://doi.org/10.1017/9781009157964.004. 


\section{Table of contents}

Executive Summary

2.1 Introduction 136

2.2 Changes in the Mountain Cryosphere 137

2.2.1 Atmospheric Drivers of Changes in the Mountain Cryosphere

Box 2.1: Does Atmospheric Warming in the Mountains Depend on Elevation?

2.2.2 Snow Cover

2.2.3 Glaciers

Cross-Chapter Box 6: Glacier Projections in Polar and High Mountain Regions

2.2.4 Permafrost 145

2.2.5 Lake and River Ice 147

Box 2.2: Local, Regional and Global Climate Feedbacks Involving the Mountain Cryosphere. 148

2.3 Mountain Social-Ecological Systems: Impacts, Risks and Human Responses 148

2.3.1 Water Resources. 148

Box 2.3: Local Responses to Water Shortage in northwest India 156

2.3.2 Landslide, Avalanche and Flood Hazards 158

Box 2.4: Challenges to Farmers and Local Population Related to Shrinkages in the Cryosphere: Cordillera Blanca, Peru 164

2.3.3 Ecosystems 165

2.3.4 Infrastructure and Mining 168

2.3.5 Tourism and Recreation 168

2.3.6 Cultural Values and Human Well-being 171

2.3.7 Migration, Habitability and Livelihoods 172
2.4 International Policy Frameworks and Pathways to Sustainable Development 173

2.5 Key Gaps in Knowledge and Prospects 174

References 176

Appendix 2.A: Additional Information on Global and Regional Glacier Mass Change Estimates for 2006-2015

Frequently Asked Questions 151

FAQ 2.1 How does glacier shrinkage affect river runoff further downhill? 151 


\section{Executive Summary}

The cryosphere (including, snow, glaciers, permafrost, lake and river ice) is an integral element of high mountain regions, which are home to roughly $10 \%$ of the global population. Widespread cryosphere changes affect physical, biological and human systems in the mountains and surrounding lowlands, with impacts evident even in the ocean. Building on the IPCC's 5th Assessment Report (AR5), this chapter assesses new evidence on observed recent and projected changes in the mountain cryosphere as well as associated impacts, risks and adaptation measures related to natural and human systems. Impacts in response to climate changes independently of changes in the cryosphere are not assessed in this chapter. Polar mountains are included in Chapter 3, except those in Alaska and adjacent Yukon, Iceland and Scandinavia, which are included in this chapter.

Observations of cryospheric changes, impacts, and adaptation in high mountain areas

Observations show general decline in low-elevation snow cover (high confidence ${ }^{1}$ ), glaciers (very high confidence) and permafrost (high confidence) due to climate change in recent decades. Snow cover duration has declined in nearly all regions, especially at lower elevations, on average by 5 days per decade, with a likely ${ }^{2}$ range from $0-10$ days per decade. Low elevation snow depth and extent have declined, although year-to-year variation is high. Mass change of glaciers in all mountain regions (excluding the Canadian and Russian Arctic, Svalbard, Greenland and Antarctica) was very likely $-490 \pm 100 \mathrm{~kg} \mathrm{~m}^{-2} \mathrm{yr}^{-1}\left(-123 \pm 24 \mathrm{Gt} \mathrm{yr}^{-1}\right)$ in 20062015. Regionally averaged mass budgets were likely most negative (less than $-850 \mathrm{~kg} \mathrm{~m}^{-2} \mathrm{yr}^{-1}$ ) in the southern Andes, Caucasus and the European Alps/Pyrenees, and least negative in High Mountain Asia $\left(-150 \pm 110 \mathrm{~kg} \mathrm{~m}^{-2} \mathrm{yr}^{-1}\right)$ but variations within regions are strong. Between 3.6-5.2 million $\mathrm{km}^{2}$ are underlain by permafrost in the eleven high mountain regions covered in this chapter corresponding to $27-29 \%$ of the global permafrost area (medium confidence). Sparse and unevenly distributed measurements show an increase in permafrost temperature (high confidence), for example, by $0.19^{\circ} \mathrm{C} \pm 0.05^{\circ} \mathrm{C}$ on average for about 28 locations in the European Alps, Scandinavia, Canada and Asia during the past decade. Other observations reveal decreasing permafrost thickness and loss of ice in the ground. $\{2.2 .2,2.2 .3,2.2 .4\}$

Glacier, snow and permafrost decline has altered the frequency, magnitude and location of most related natural hazards (high confidence). Exposure of people and infrastructure to natural hazards has increased due to growing population, tourism and socioeconomic development (high confidence). Glacier retreat and permafrost thaw have decreased the stability of mountain slopes and the integrity of infrastructure (high confidence). The number and area of glacier lakes has increased in most regions in recent decades (high confidence), but there is only limited evidence that the frequency of glacier lake outburst floods (GLOF) has changed. In some regions, snow avalanches involving wet snow have increased (medium confidence), and rain-on-snow floods have decreased at low elevations in spring and increased at high elevations in winter (medium confidence). The number and extent of wildfires have increased in the Western USA partly due to early snowmelt (medium confidence). $\{2.3 .2,2.3 .3\}$

Changes in snow and glaciers have changed the amount and seasonality of runoff in snow-dominated and glacier-fed river basins (very high confidence) with local impacts on water resources and agriculture (medium confidence). Winter runoff has increased in recent decades due to more precipitation falling as rain (high confidence). In some glacier-fed rivers, summer and annual runoff have increased due to intensified glacier melt, but decreased where glacier melt water has lessened as glacier area shrinks. Decreases were observed especially in regions dominated by small glaciers, such as the European Alps (medium confidence). Glacier retreat and snow cover changes have contributed to localized declines in agricultural yields in some high mountain regions, including the Hindu Kush Himalaya and the tropical Andes (medium confidence). There is limited evidence of impacts on operation and productivity of hydropower facilities resulting from changes in seasonality and both increases and decreases in water input, for example, in the European Alps, Iceland, Western Canada and USA, and the tropical Andes. $\{2.3 .1\}$

Species composition and abundance have markedly changed in high mountain ecosystems in recent decades (very high confidence), partly due to changes in the cryosphere (high confidence). Habitats for establishment by formerly absent species have opened up or been altered by reduced snow cover (high confidence), retreating glaciers (very high confidence), and thawing of permafrost (medium confidence). Reductions in glacier and snow cover have directly altered the structure of many freshwater communities (high confidence). Reduced snow cover has negatively impacted the reproductive fitness of some snow-dependent plant and animal species, including foraging and predator-prey relationships of mammals (high confidence). Upslope migration of individual species, mostly due to warming and to a lesser extent due to cryosphere-related changes, has often increased local species richness (very high confidence). Some cold-adapted species, including endemics, in terrestrial and freshwater communities have declined

1 In this report, the following summary terms are used to describe the available evidence: limited, medium, or robust; and for the degree of agreement: low, medium or high. A level of confidence is expressed using five qualifiers: very low, low, medium, high and very high, and typeset in italics, for example, medium confidence. For a given evidence and agreement statement, different confidence levels can be assigned, but increasing levels of evidence and degrees of agreement are correlated with increasing confidence (see Section 1.9.2 and Figure 1.4 for more details).

2 In this report, the following terms have been used to indicate the assessed likelihood of an outcome or a result: Virtually certain 99-100\% probability, Very likely 90-100\%, Likely $66-100 \%$, About as likely as not 33-66\%, Unlikely 0-33\%, Very unlikely 0-10\% and Exceptionally unlikely 0-1\%. Additional terms (Extremely likely: $95-100 \%$, More likely than not $>50-100 \%$ and Extremely unlikely 0-5\%) may also be used when appropriate. Assessed likelihood is typeset in italics, for example, very likely (see Section 1.9.2 and Figure 1.4 for more details). This Report also uses the term 'likely range' to indicate that the assessed likelihood of an outcome lies within the 17-83\% probability range. 
in abundance (high confidence). While the plant productivity has generally increased, the actual impact on provisioning, regulating and cultural ecosystem services varies greatly (high confidence). $\{2.3 .3\}$

Tourism and recreation activities such as skiing, glacier tourism and mountaineering have been negatively impacted by declining snow cover, glaciers and permafrost (medium confidence). In several regions, worsening route safety has reduced mountaineering opportunities (medium confidence). Variability and decline in natural snow cover have compromised the operation of low-elevation ski resorts (high confidence). Glacier and snow decline have impacted aesthetic, spiritual and other cultural aspects of mountain landscapes (medium confidence), reducing the well-being of people (e.g., in the Himalaya, eastern Africa, and the tropical Andes). $\{2.3 .5,2.3 .6\}$

Adaptation in agriculture, tourism and drinking water supply has aimed to reduce the impacts of cryosphere change (medium confidence), though there is limited evidence on their effectiveness owing to a lack of formal evaluations, or technical, financial and institutional barriers to implementation. In some places, artificial snowmaking has reduced the negative impacts on ski tourism (medium confidence). Release and storage of water from reservoirs according to sectoral needs (agriculture, drinking water, ecosystems) has reduced the impact of seasonal variability on runoff (medium confidence). $\{2.3 .1,2.3 .5\}$

Future projections of cryospheric changes, their impacts and risks, and adaptation in high mountain areas

Snow cover, glaciers and permafrost are projected to continue to decline in almost all regions throughout the 21st century (high confidence). Compared to 1986-2005, low elevation snow depth will likely decrease by $10-40 \%$ for $2031-2050$, regardless of Representative Concentration Pathway (RCP) and for 2081-2100, likely by $10-40 \%$ for RCP2.6 and by $50-90 \%$ for RCP8.5. Projected glacier mass reductions between 2015-2100 are likely 22-44\% for RCP2.6 and $37-57 \%$ for RCP8.5. In regions with mostly smaller glaciers and relatively little ice cover (e.g., European Alps, Pyrenees, Caucasus, North Asia, Scandinavia, tropical Andes, Mexico, eastern Africa and Indonesia), glaciers will lose more than $80 \%$ of their current mass by 2100 under RCP8.5 (medium confidence), and many glaciers will disappear regardless emission scenario (very high confidence). Permafrost thaw and degradation will increase during the 21st century (very high confidence) but quantitative projections are scarce. $\{2.2 .2,2.2 .3,2.2 .4\}$

Most types of natural hazards are projected to change in frequency, magnitude and areas affected as the cryosphere continues to decline (high confidence). Glacier retreat and permafrost thaw are projected to decrease the stability of mountain slopes and increase the number and area of glacier lakes (high confidence). Resulting landslides and floods, and cascading events, will also emerge where there is no record of previous events (high confidence). Snow avalanches are projected to decline in number and runout distance at lower elevation, and avalanches involving wet snow even in winter will occur more frequently (medium confidence). Rain-on-snow floods will occur earlier in spring and later in autumn, and be more frequent at higher elevations and less frequent at lower elevations (high confidence). $\{2.3 .2,2.3 .3\}$

River runoff in snow dominated and glacier-fed river basins will change further in amount and seasonality in response to projected snow cover and glacier decline (very high confidence) with negative impacts on agriculture, hydropower and water quality in some regions (medium confidence). The average winter snowmelt runoff is projected to increase (high confidence), and spring peaks to occur earlier (very high confidence). Projected trends in annual runoff vary substantially among regions, and can even be opposite in direction, but there is high confidence that in all regions average annual runoff from glaciers will have reached a peak that will be followed by declining runoff at the latest by the end of the 21st century. Declining runoff is expected to reduce the productivity of irrigated agriculture in some regions (medium confidence). Hydropower operations will increasingly be impacted by altered amount and seasonality of water supply from snow and glacier melt (high confidence). The release of heavy metals, particularly mercury, and other legacy contaminants currently stored in glaciers and permafrost, is projected to reduce water quality for freshwater biota, household use and irrigation (medium confidence). $\{2.3 .1\}$

Current trends in cryosphere-related changes in high mountain ecosystems are expected to continue and impacts to intensify (very high confidence). While high mountains will provide new and greater habitat area, including refugia for lowland species, both range expansion and shrinkage are projected, and at high elevations this will lead to population declines (high confidence). The latter increases the risk of local extinctions, in particular for freshwater cold-adapted species (medium confidence). Without genetic plasticity and/or behavioural shifts, cryospheric changes will continue to negatively impact endemic and native species, such as some coldwater fish (e.g., trout) and species whose traits directly depend on snow (e.g., snowshoe hares) or many large mammals (medium confidence). The survival of such species will depend on appropriate conservation and adaptation measures (medium confidence). Many projected ecological changes will alter ecosystem services (high confidence), affecting ecological disturbances (e.g., fire, rock fall, slope erosion) with considerable impacts on people (medium confidence). $\{2.3 .3\}$

Cultural assets, such as snow- and ice-covered peaks in many UNESCO World Heritage sites, and tourism and recreation activities, are expected to be negatively affected by future cryospheric change in many regions (high confidence). Current snowmaking technologies are projected to be less effective in a warmer climate in reducing risks to ski tourism in most parts of Europe, North America and Japan, in particular at $2^{\circ} \mathrm{C}$ global warming and beyond (high confidence). Diversification through year-round activities supports adaptation of tourism under future climate change (medium confidence). $\{2.3 .5,2.3 .6\}$ 
Enablers and response options to promote adaptation and sustainable development in high mountain areas

The already committed and unavoidable climate change affecting all cryosphere elements, irrespective of the emission scenario, points to integrated adaptation planning to support and enhance water availability, access, and management (medium confidence). Integrated management approaches for water across all scales, in particular for energy, agriculture, ecosystems and drinking water supply, can be effective at dealing with impacts from changes in the cryosphere. These approaches also offer opportunities to support social-ecological systems, through the development and optimisation of storage and the release of water from reservoirs (medium confidence), while being cognisant of potential negative implications for some ecosystems. Success in implementing such management options depends on the participation of relevant stakeholders, including affected communities, diverse knowledge and adequate tools for monitoring and projecting future conditions, and financial and institutional resources to support planning and implementation (medium confidence). $\{2.3 .1,2.3 .3,2.4\}$

Effective governance is a key enabler for reducing disaster risk, considering relevant exposure factors such as planning, zoning, and urbanisation pressures, as well as vulnerability factors such as poverty, which can challenge efforts towards resilience and sustainable development for communities (medium confidence). Reducing losses to disasters depend on integrated and coordinated approaches to account for the hazards concerned, the degree of exposure, and existing vulnerabilities. Diverse knowledge that includes community and multi-stakeholder experience with past impacts complements scientific knowledge to anticipate future risks. \{CCB-1, 2.3.2, 2.4\}

International cooperation, treaties and conventions exist for some mountain regions and transboundary river basins with potential to support adaptation action. However, there is limited evidence on the extent to which impacts and losses arising from changes in the cryosphere are specifically monitored and addressed in these frameworks. A wide range of institutional arrangements and practices have emerged over the past three decades that respond to a shared global mountain agenda and specific regional priorities. There is potential to strengthen them to also respond to climate-related cryosphere risks and open opportunities for development through adaptation (limited evidence, high agreement). The Sustainable Development Goals (SDGs), Sendai Framework and Paris Agreement have directed some attention in mountain-specific research and practice towards the monitoring and reporting on targets and indicators specified therein. $\{2.3 .1,2.4\}$ 


\section{$2.1 \quad$ Introduction}

High mountain regions share common features, including rugged terrain, a low-temperature climate regime, steep slopes and institutional and spatial remoteness. These features are often linked to physical and social-ecological processes that, although not unique to mountain regions, typify many of the special aspects of these regions. Due to their higher elevation compared with the surrounding landscape, mountains often feature cryosphere components, such as glaciers, snow cover and permafrost, with a significant influence on surrounding lowland areas even far from the mountains (Huggel et al., 2015a). Hence the mountain cryosphere plays a major role in large parts of the world. Considering the close relationship between mountains and the cryosphere, high mountain areas are addressed
(671 million people) of the global population lived in high mountain regions in 2010, based on gridded population data (Jones and O'Neill, 2016) and a distance of less than $100 \mathrm{~km}$ from glaciers or permafrost located in mountains areas as defined in Figure 2.1. This population is expected to grow to 736-844 million across the shared socioeconomic pathways by 2050 (Gao, 2019). Many people living outside of mountain areas and not included in these numbers are also affected by changes in the mountain cryosphere.

This chapter assesses recent and projected changes in glaciers, snow cover, permafrost and lake and river ice in high mountain areas, their drivers, as well as their impact on the different services provided by the cryosphere and related adaptation, with a focus on literature published after AR5. The assessment of cryospheric change is focused on recent decades rather than a perspective over a longer period,

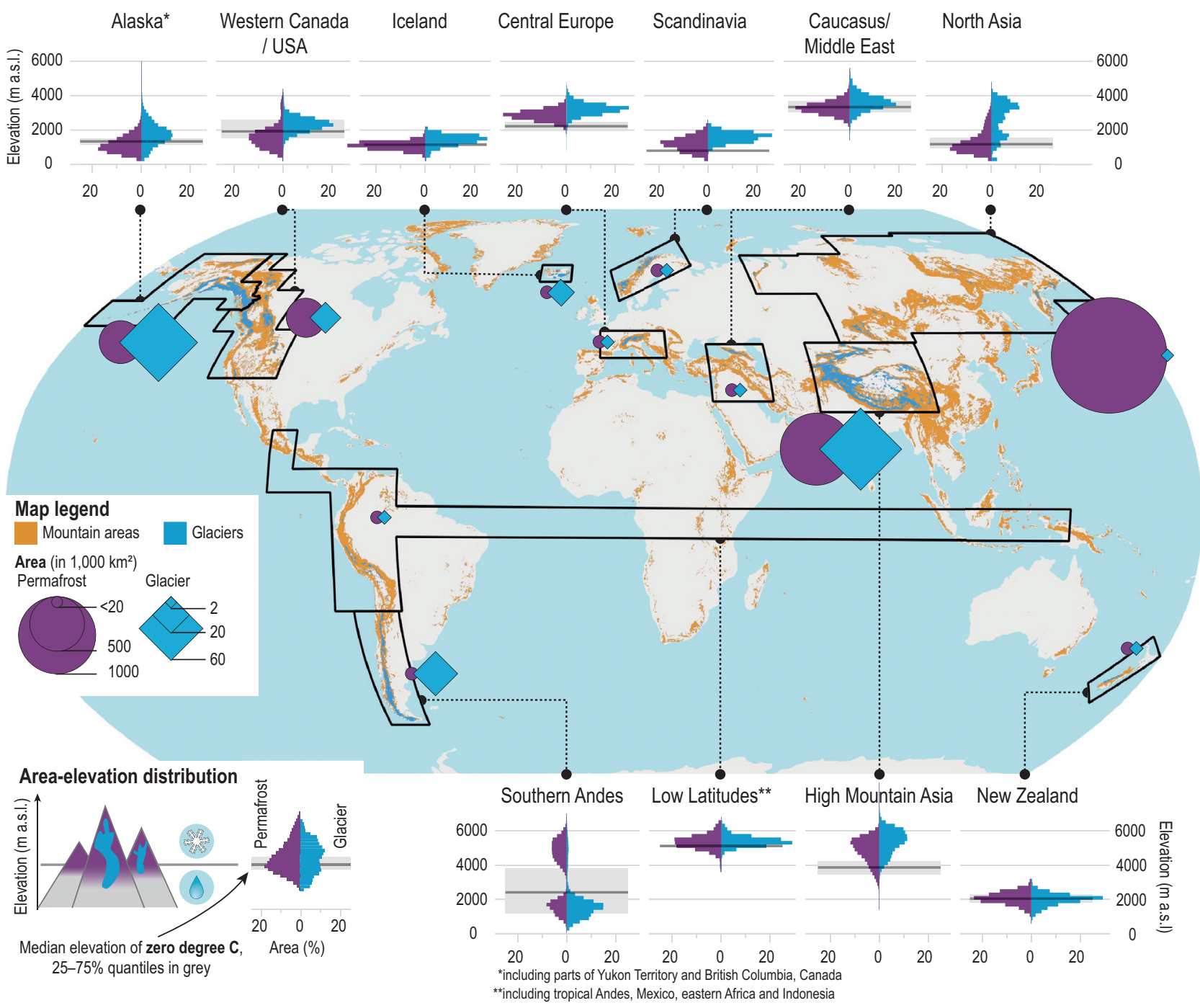

Figure 2.1 Distribution of mountain areas (orange shading) and glaciers (blue) as well as regional summary statistics for glaciers and permafrost in mountains. Mountains are distinguished based on a ruggedness index (>3.5), a logarithmically scaled measure of relative relief (Gruber, 2012). Eleven distinct regions with glaciers, generally corresponding to the primary regions in the Randolph Glacier Inventory, RGI v6.0 (RGI Consortium, 2017) are outlined, although some cryosphere related impacts presented in this chapter may go beyond these regions. Region names correspond to those in the RGI. Diamonds represent regional glacier area (RGI 6.0) and circles the permafrost area in all mountains within each region boundary (Obu et al., 2019). Histograms for each region show glacier and permafrost area in $200 \mathrm{~m}$ elevation bins as a percentage of total regional glacier and permafrost area, respectively. Also shown is the median elevation of the annual mean $0^{\circ} \mathrm{C}$ free-atmosphere isotherm calculated from the ERA-5 re-analysis of the European Centre for Medium Range Weather Forecasts over each region's mountain area for the period $2006-2015$, with $25-75 \%$ quantiles in grey. The annual $0^{\circ} \mathrm{C}$ isotherm elevation roughly separates the areas where precipitation predominantly falls as snow and rain. Areas above and below this elevation are loosely referred to as high and low elevations, respectively, in this chapter. 
and future changes spanning the 21st century. A palaeo-perspective is covered in IPCC Sixth Assessment Report (AR6) Working Group I contribution on 'The Physical Science Basis'. High mountain areas, as discussed here, include all mountain regions where glaciers, snow or permafrost are prominent features of the landscape, without a strict and quantitative demarcation, but with a focus on distinct regions (Figure 2.1). Mountain regions located in the polar regions are considered in Chapter 3 except those in Iceland, Scandinavia and Alaska and parts of adjacent Yukon Territory and British Columbia, which are included in this chapter. Many changes in the mountain environment are not solely or directly related to climate change induced changes in the cryosphere, but to other direct or indirect effects of climate change, or to other consequences of socioeconomic development. Consistent with the scope of this report with a focus on the ocean and the cryosphere, this section deals primarily with the impacts that can at least partially be attributed to cryosphere changes. Even though other drivers may be the dominant driver of change in many cases, they are not considered explicitly in this chapter, although unambiguous attribution to cryosphere changes is often difficult.

\subsection{Changes in the Mountain Cryosphere}

\subsubsection{Atmospheric Drivers of Changes in the Mountain Cryosphere}

Past changes of surface air temperature and precipitation in high mountain areas have been documented by in situ observations and regional reanalyses (Table SM2.2 and Table SM2.4). However, mountain observation networks do not always follow standard measurement procedures (Oyler et al., 2015; Nitu et al., 2018) and are often insufficiently dense to capture fine-scale changes (Lawrimore et al., 2011) and the underlying larger scale patterns. Future changes are projected using General Circulation Models (GCMs) or Regional Climate Models (RCMs) or simplified versions thereof (e.g., Gutmann et al., 2016), used to represent processes at play in a dynamically consistent manner, and to relate mountain changes to larger-scale atmospheric forcing based on physical principles. Existing mountain-specific model studies typically cover individual mountain ranges, and there is currently no initiative found, such as model intercomparisons or coordinated model experiments, which specifically and comprehensively addresses high mountain meteorology and climate globally. This makes it difficult to provide a globally uniform assessment.

\subsubsection{Surface Air Temperature}

Mountain surface air temperature observations in Western North America, European Alps and High Mountain Asia show warming over recent decades at an average rate of $0.3^{\circ} \mathrm{C}$ per decade, with a likely range of $\pm 0.2^{\circ} \mathrm{C}$, thereby outpacing the global warming rate $0.2 \pm 0.1^{\circ} \mathrm{C}$ per decade (IPCC, 2018). Underlying data from global and regional studies are compiled in Table SM2.2, and Figure 2.2 provides a synthesis on mountain warming trends, mostly based on studies using in situ observations. Local warming rates depend on the season (high confidence). For example, in the European Alps,

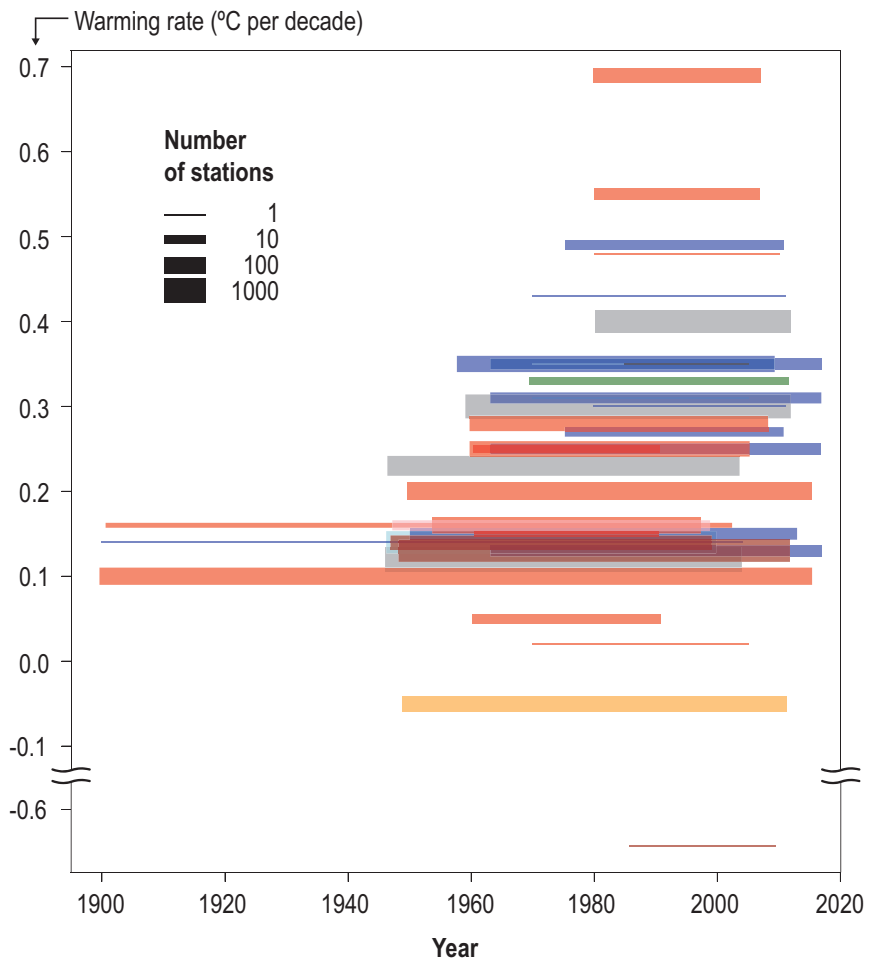

Figure 2.2 Synthesis of trends in mean annual surface air temperature in mountain regions, based on 4672 observation stations (partly overlapping) aggregated in 38 datasets reported in 19 studies. Each line refers to a warming rate from one dataset, calculated over the time period indicated by the extent of the line. Colours indicate mountain region (Figure 2.1), and line thickness the number of observation stations used. Detailed references are found in Table SM2.2, which also provides additional information on trends for individual seasons and other temperature indicators (daily minimum or maximum temperature).

warming has been found to be more pronounced in summer and spring (Auer et al., 2007; Ceppi et al., 2012), while on the Tibetan Plateau warming is stronger in winter (Liu et al., 2009; You et al., 2010). Studies comparing observations at lower and higher elevation at the global scale indicate that warming is generally enhanced above $500 \mathrm{~m}$ above sea level (a.s.I.) (e.g., Wang et al., 2016a; Qixiang et al., 2018, Table SM2.2). At the local and regional scale, evidence for elevation dependent warming, i.e., that the warming rate is different across elevation bands, is scattered and sometimes contradictory (Box 2.1). On the Tibetan Plateau, evidence based on combining in situ observations (often scarce at high elevation) with remote sensing and modelling approaches, indicates that warming is amplified around 4,000 $\mathrm{m}$ a.s.l., but not above 5,000 $\mathrm{m}$ a.s.l. (Qin et al., 2009; Gao et al., 2018). Studies in the Italian Alps (Tudoroiu et al., 2016) and Southern Himalaya (Nepal, 2016) have shown higher warming at lower elevation. Evidence from Western North and South America is conflicting (Table SM2.2). In other regions, evidence to assess whether warming varies with elevation is insufficient. In summary, there is medium evidence (medium agreement) that surface warming is different across elevation bands. Observed changes also depend on the type of temperature indicator: changes in daily mean, minimum 


\section{Box 2.1 Does Atmospheric Warming in the Mountains Depend on Elevation?}

In mountain regions, surface air temperature generally tends to decrease with increasing elevation thus directly impacting how much of the precipitation falls as snow as opposed to rain. Therefore, changes in air temperature have different consequences for snow cover, permafrost and glaciers at different elevations. A number of studies have reported that trends in air temperature vary with elevation, a phenomenon referred to as elevation dependent warming (EDW; Pepin et al., 2015, and references therein), with potential consequences beyond those of uniform warming. EDW does not imply that warming is larger at higher elevation, and smaller at lower elevation, but it means that the warming rate (e.g., in ${ }^{\circ} \mathrm{C}$ per decade) is not the same across all elevation bands. Although this concept has received wide attention in recent years, the manifestation of EDW varies by region, season and temperature indicator (e.g., daily mean, minimum or maximum temperature), meaning that a uniform pattern does not exist. The identification of the underlying driving mechanisms for EDW and how they combine is complex.

Several physical processes contribute to EDW, and quantifying their relative contributions has remained largely elusive (Minder et al., 2018; Palazzi et al., 2019). Some of the processes identified are similar to those explaining the amplified warming in the polar regions (Chapter 3). For example, the sensitivity of temperature to radiative forcing is increased at low temperatures common in both polar and mountain environments (Ohmura, 2012). Because the relationship between specific humidity and downwelling radiation is nonlinear, in a dry and cold atmosphere found at high elevation, any increase in atmospheric humidity due to temperature increase drives disproportionately large warming (Rangwala et al., 2013; Chen et al., 2014). Snow-albedo feedback plays an important role where the snow cover is in decline (Pepin and Lundquist, 2008; Scherrer et al., 2012), increasing the absorption of solar radiation which in turn leads to increased surface air temperature and further snowmelt. Other processes are specific to the mountain environment. Especially in the tropics, warming can be enhanced at higher elevation by a reduction of the vertical temperature gradient, due to increased latent heat release above the condensation level, favored in a warmer and moister atmosphere (Held and Soden, 2006). The cooling effect of aerosols, which also cause solar dimming, is more pronounced at low elevation and reduced at high elevation (Zeng et al., 2015). While many mechanisms suggest that warming should be enhanced at high elevation, observed and simulated EDW patterns are usually more complex (Pepin et al., 2015, and references therein). Numerical simulations by global and regional climate models, which show EDW, need to be considered carefully because of intrinsic limitations due to potentially incomplete understanding and implementation of relevant physical processes, in addition to coarse grid spacing with respect to mountainous topography (Ménégoz et al., 2014; Winter et al., 2017).

and maximum temperature can display contrasting patterns depending on region, season and elevation (Table SM2.2).

Attribution studies for changes in surface air temperature specifically in mountain regions are rare. Bonfils et al. (2008) and Dileepkumar et al. (2018) demonstrated that anthropogenic greenhouse gas emissions are the dominant factor in the recent temperature increases, partially compensated by other anthropogenic factors (land use change and aerosol emissions for Western USA and Western Himalaya, respectively). These findings are consistent with conclusions of AR5 regarding anthropogenic effects (Bindoff et al., 2013). It is thus likely that anthropogenic influence is the main contributor to surface temperature increases in high mountain regions since the mid-20th century, amplified by regional feedbacks.

Until the mid-21st century, regardless of the climate scenario (Cross-Chapter Box 1 in Chapter 1), surface air temperature is projected to continue increasing (very high confidence) at an average rate of $0.3^{\circ} \mathrm{C}$ per decade, with a likely range of $\pm 0.2^{\circ} \mathrm{C}$ per decade, locally even more in some regions, generally outpacing global warming rates $\left(0.2 \pm 0.1^{\circ} \mathrm{C}\right.$ per decade; IPCC, 2018) (high confidence). Beyond mid-21st century, atmospheric warming in mountains will be stronger under a high greenhouse gas emission scenario (RCP8.5) and will stabilise at mid-21st levels under a low greenhouse gas emission scenario (RCP2.6), similar to global change patterns (very high confidence). The warming rate will result from the combination of regional (high confidence) and elevation-dependent (medium confidence) enhancement factors. Underlying evidence of future projections from global and regional studies is provided in Table SM2.3. Figure 2.3 provides examples of regional climate projections of surface air temperature, as a function of elevation and season (winter and summer) in North America (Rocky Mountains), South America (Subtropical Central Andes), Europe (European Alps) and High Mountain Asia (Hindu Kush, Karakoram, Himalaya), based on global and regional climate projections.

\subsubsection{Rainfall and Snowfall}

Past precipitation changes are less well quantified than temperature changes and are often more heterogeneous, even within mountain regions (Hartmann and Andresky, 2013). Regional patterns are characterised by decadal variability (Mankin and Diffenbaugh, 2015) and influenced by shifts in large-scale atmospheric circulation (e.g., in Alaska; Winski et al., 2017). While mountain regions do not exhibit clear direction of trends in annual precipitation over the past decades (medium confidence that there is no trend), snowfall has decreased, at least in part due to higher temperatures, especially at lower elevation (Table SM2.4, high confidence).

Future projections of annual precipitation indicate increases of the order of 5 to $20 \%$ over the 21 st century in many mountain regions, including the Hindu Kush and Himalaya, East Asia, eastern Africa, 
the European Alps and the Carpathian region, and decreases in the Mediterranean and the Southern Andes (medium confidence, Table SM2.5). Changes in the frequency and intensity of extreme precipitation events vary according to season and region. For example, across the Himalayan-Tibetan Plateau mountains, the frequency and intensity of extreme rainfall events are projected to increase throughout the 21st century, particularly during the summer monsoon (Panday et al., 2015; Sanjay et al., 2017). This suggests a transition toward more episodic and intense monsoonal precipitation, especially in the easternmost part of the Himalayan chain (Palazzi et al., 2013). Increases in winter precipitation extremes are projected in the European Alps (Rajczak and Schär, 2017). At lower elevation, near term (2031-2050) and end of century (2081-2100) projections of snowfall all indicate a decrease, for all greenhouse gas emission scenarios (very high confidence). At higher elevation, where temperature increase is insufficient to affect rain/ snow partitioning, total winter precipitation increases can lead to increased snowfall (e.g., Kapnick and Delworth, 2013; 0'Gorman, 2014) (medium confidence).

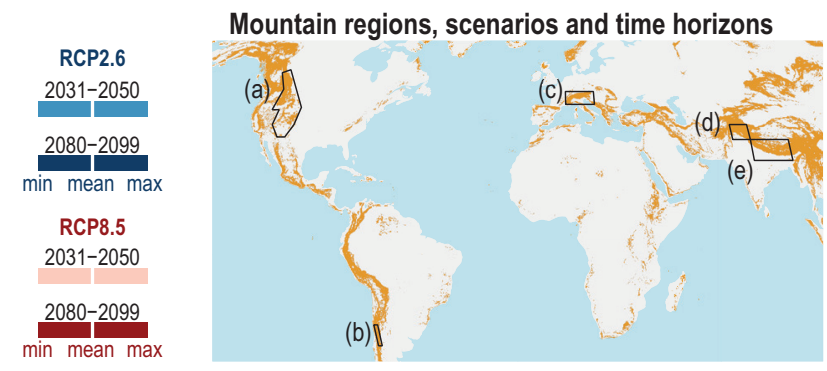

(a) Rocky Mountains

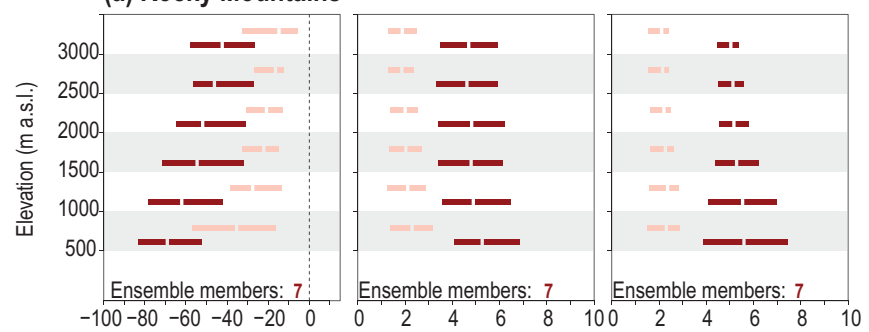

(b) Subtropical Central Andes

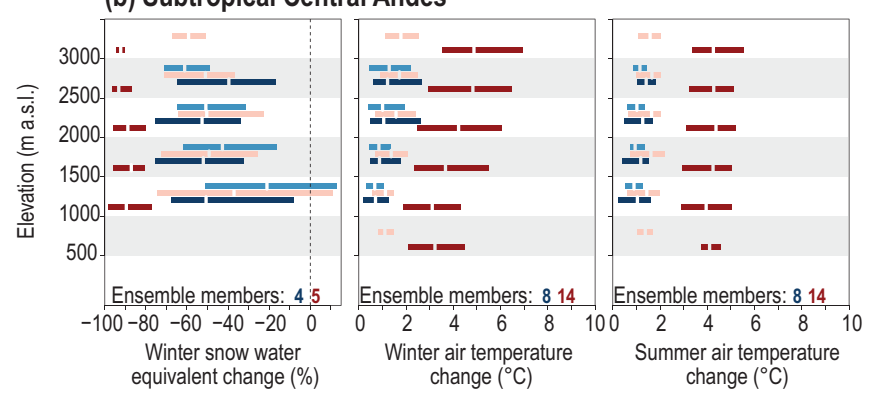

\subsubsection{Other Meteorological Variables}

Atmospheric humidity, incoming shortwave and longwave radiation, and near-surface wind speed and direction also influence the high mountain cryosphere. Detecting their changes and associated effects on the cryosphere is even more challenging than for surface air temperature and precipitation, both from an observation and modelling standpoint. Therefore, most simulation studies of cryosphere changes are mainly driven by temperature and precipitation (see, e.g., Beniston et al., 2018, and references therein).

Atmospheric moisture content, which is generally increasing in a warming atmosphere (Stocker et al., 2013), affects latent and longwave heat fluxes (Armstrong and Brun, 2008) with implications for the timing and rate of snow and ice ablation, and in some areas changes in atmospheric moisture content could be a significant driver of cryosphere change (Harpold and Brooks, 2018). Short-lived climate forcers, such as sulphur and black carbon aerosols (You et al., 2013), reduce the amount of solar radiation reaching the surface, with potential impacts on snow and ice ablation. Solar brightening caused by declining anthropogenic aerosols in Europe since the 1980s was

\section{(c) European Alps}

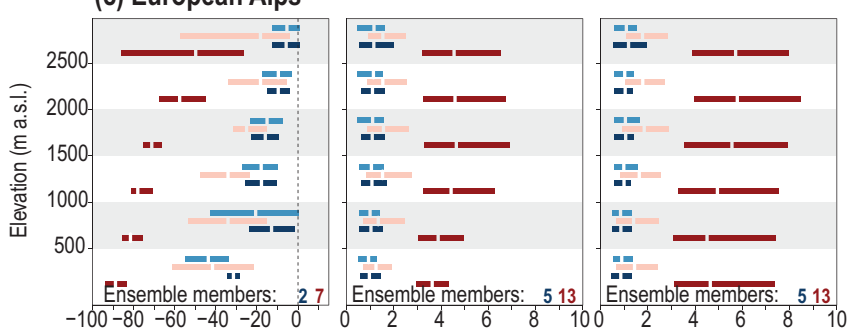

(d) Hindu Kush and Karakoram

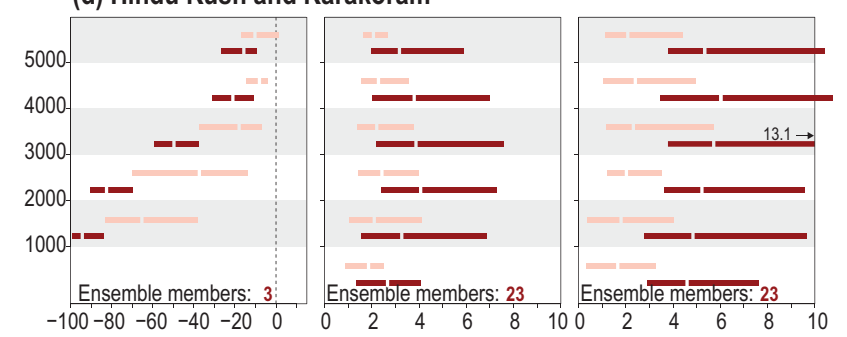

(e) Himalaya

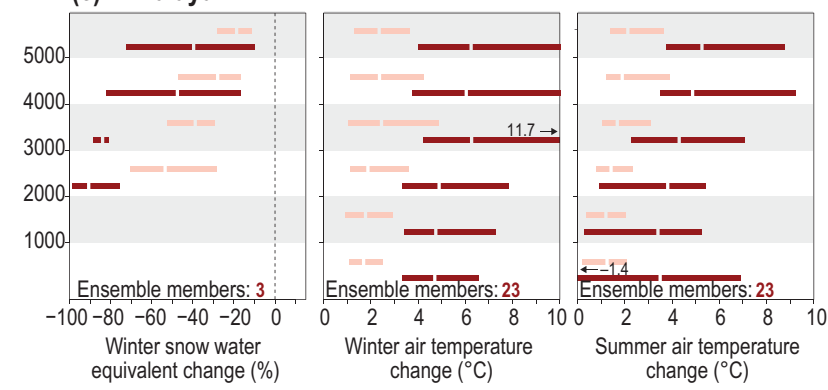

Figure 2.3 Projected change (1986-2005 to 2031-2050 and 2080-2099) of mean winter (December to May; June to August in Subtropical Central Andes) snow water equivalent, winter air temperature and summer air temperature (June to August; December to February in Subtropical Central Andes) in five high mountain regions for RCP8.5 (all regions) and RCP2.6 (European Alps and Subtropical Central Andes). Changes are averaged over $500 \mathrm{~m}(\mathrm{a}, \mathrm{b}, \mathrm{c})$ and 1,000 m (d,e) elevation bands. The numbers in the lower right of each panel reflect the number of simulations (note that not all models provide snow water equivalent). For the Rocky Mountains, data from NA-CORDEX RCMs (25 km grid spacing) driven by Coupled Model Intercomparison Project Phase 5 (CMIP5) General Circulation Models (GCMs) were used (Mearns et al., 2017). For the European Alps, data from EURO-CORDEX RCMs (12 km grid spacing) driven by CMIP5 GCMs were used (Jacob et al., 2014). For the other regions, CMIP5 GCMs were used: Zazulie (2016) and Zazulie et al. (2018) for the Subtropical Central Andes, and Terzago et al. (2014) and Palazzi et al. (2017) for the Hindu Kush and Karakoram and Himalaya. The list of models used is provided in Table SM2.8. 
shown to have only a minor effect on atmospheric warming at high elevation (Philipona, 2013), and effects on the cryosphere were not specifically discussed.

Wind controls preferential deposition of precipitation, postdepositional snow drift and affects ablation of snow and glaciers through turbulent fluxes. Near-surface wind speed has decreased on the Tibetan Plateau between the 1970s and early 2000s, and stabilised or increased slightly thereafter (Yang et al., 2014a; Kuang and Jiao, 2016). This is consistent with existing evidence for a decrease in near-surface wind speed on mid-latitude continental areas since the mid-20th century (Hartmann et al., 2013). In general, the literature on past and future changes of near-surface wind patterns in mountain areas is very limited.

\subsubsection{Snow Cover}

Snow on the ground is an essential and widespread component of the mountain cryosphere. It affects mountain ecosystems and plays a major role for mass movement and floods in the mountains. It plays a key role in nourishing glaciers and provides an insulating and reflective cover at their surface. It influences the thermal regime of the underlying ground, including permafrost, with implications for ecosystems. Climate change modifies key variables driving the onset and development of the snow cover (e.g., solid precipitation), and those responsible for its ablation (e.g., air temperature, radiation). The snow cover, especially in low-lying and mid-elevation areas of mountain regions, has long been identified to be particularly sensitive to climate change.

The mountain snow cover is characterised by a very strong interannual and decadal variability, similar to its main driving force solid precipitation (Lafaysse et al., 2014; Mankin and Diffenbaugh, 2015). Observations spanning several decades are required to quantify trends. Long-term in situ records are scarce in some regions of the world, particularly in High Mountain Asia, Northern Asia and South America (Rohrer et al., 2013). Satellite remote sensing provides new capabilities for monitoring mountain snow cover on regional scales. The satellite record length is often insufficient to assess trends (Bormann et al., 2018). Evidence of past changes from regional studies is provided in Table SM2.6. At lower elevation, there is high confidence that the mountain snow cover has generally declined in duration (on average by 5 snow cover days per decade, with a likely range from 0 to 10 days per decade), mean snow depth and accumulated mass (snow water equivalent) since the middle of the 20 th century, with regional variations. At higher elevation, snow cover trends are generally insignificant (medium confidence) or unknown.

Most of the snow cover changes can be attributed, at lower elevation, to more precipitation falling as liquid precipitation (rain) and to increases in melt at all elevations, mostly due to changes in atmospheric forcings, especially increased air temperature (Kapnick and Hall, 2012; Marty et al., 2017) which in turn are attributed to anthropogenic forcings at a larger scale (Section 2.2.1). Formal anthropogenic attribution studies provide similar conclusions in Western North America (Pierce et al., 2008; Najafi et al., 2017).
Assessing the impact of the deposition of short-lived climate forcers on snow cover changes is an emerging issue (Skiles et al., 2018 and references therein). This concerns light absorbing particles, in particular, which include deposited aerosols such as black carbon, organic carbon and mineral dust, or microbial growth (Qian et al., 2015), although the role of the latter has not been specifically quantified. Due to their seasonally variable deposition flux and impact, and mostly episodic nature in case of dust deposition (Kaspari et al., 2014; Di Mauro et al., 2015), light absorbing particles contribute to interannual fluctuations of seasonal snowmelt rate (Painter et al., 2018) (medium evidence, high agreement). There is limited evidence (medium agreement) that increases in black carbon deposition from anthropogenic and biomass burning sources have contributed to snow cover decline in High Mountain Asia (Li et al., 2016; Zhang et al., 2018) and South America (Molina et al., 2015).

Projected changes of mountain snow cover are studied based on climate model experiments, either directly from GCM or RCM output, or following downscaling and the use of snowpack models. These projections generally do not specifically account for future changes in the deposition rate of light absorbing particles on snow (or, if so, simple approaches have been used hitherto; e.g., Deems et al., 2013), so that future changes in snow conditions are mostly driven by changes in meteorological drivers assessed in Section 2.2.1. Evidence from regional studies is provided in Table SM2.7. Although existing studies in mountain regions do not use homogenous reference periods and model configurations, common future trends can be summarised as follows. At lower elevation in many regions such as the European Alps, Western North America, Himalaya and subtropical Andes, the snow depth or mass is projected to decline by 25\% (likely range between 10 and $40 \%$ ), between the recent past period (1986-2005) and the near future (2031-2050), regardless of the greenhouse gas emission scenario (Cross-Chapter Box 1 in Chapter 1). This corresponds to a continuation of the ongoing decrease in annual snow cover duration (on average 5 days per decade, with a likely range from 0 to 10). By the end of the century (2081-2100), reductions of up to $80 \%$ (likely range from 50 to $90 \%$ ) are expected under RCP8.5, 50\% (likely range from 30 to $70 \%$ ) under RCP4.5 and 30\% (likely range from 10 to $40 \%$ ) under RCP2.6. At higher elevations, projected reductions are smaller (high confidence), as temperature increases at higher elevations affect the ablation component of snow mass evolution, rather than both the onset and accumulation components. The projected increase in winter snow accumulation may result in a net increase in winter snow mass (medium confidence). All elevation levels and mountain regions are projected to exhibit sustained interannual variability of snow conditions throughout the 21 st century (high confidence). Figure 2.3 provides projections of temperature and snow cover in mountain areas in Europe, High Mountain Asia (Hindu Kush, Karakoram and Himalaya), North America (Rocky Mountains) and South America (sub-tropical Central Andes), illustrating how changes vary with elevation, season, region, future time period and climate scenario. 


\subsubsection{Glaciers}

The high mountain areas considered in this chapter (Figure 2.1), including all glacier regions in the world except those in Antarctica, Greenland, the Canadian and Russian Arctic, and Svalbard (which are covered in Chapter 3) include $\sim 170,000$ glaciers covering an area of $\sim 250,000 \mathrm{~km}^{2}$ (RGI Consortium, 2017) with a total ice volume of $87 \pm 15 \mathrm{~mm}$ sea level equivalent (Farinotti et al., 2019). These glaciers span an elevation range from sea level, for example in south-east Alaska, to $>8,000 \mathrm{~m}$ a.s.l. in the Himalaya and Karakoram, and occupy diverse climatic regions. Their mass budget is determined largely by the balance between snow accumulation and melt at the glacier surface, driven primarily by atmospheric conditions. Rapid changes in mountain glaciers have multiple impacts for social-ecological systems, affecting not only biophysical properties such as runoff volume and sediment fluxes in glacier-fed rivers, glacier related hazards, and global sea level (Chapter 4) but also ecosystems and human livelihoods, socioeconomic activities and sectors such as agriculture and tourism, as well as other intrinsic assets such as cultural values. While glaciers worldwide have experienced considerable fluctuations throughout the Holocene driven by multidecadal variations of solar and volcanic activity, and changes in atmospheric circulation
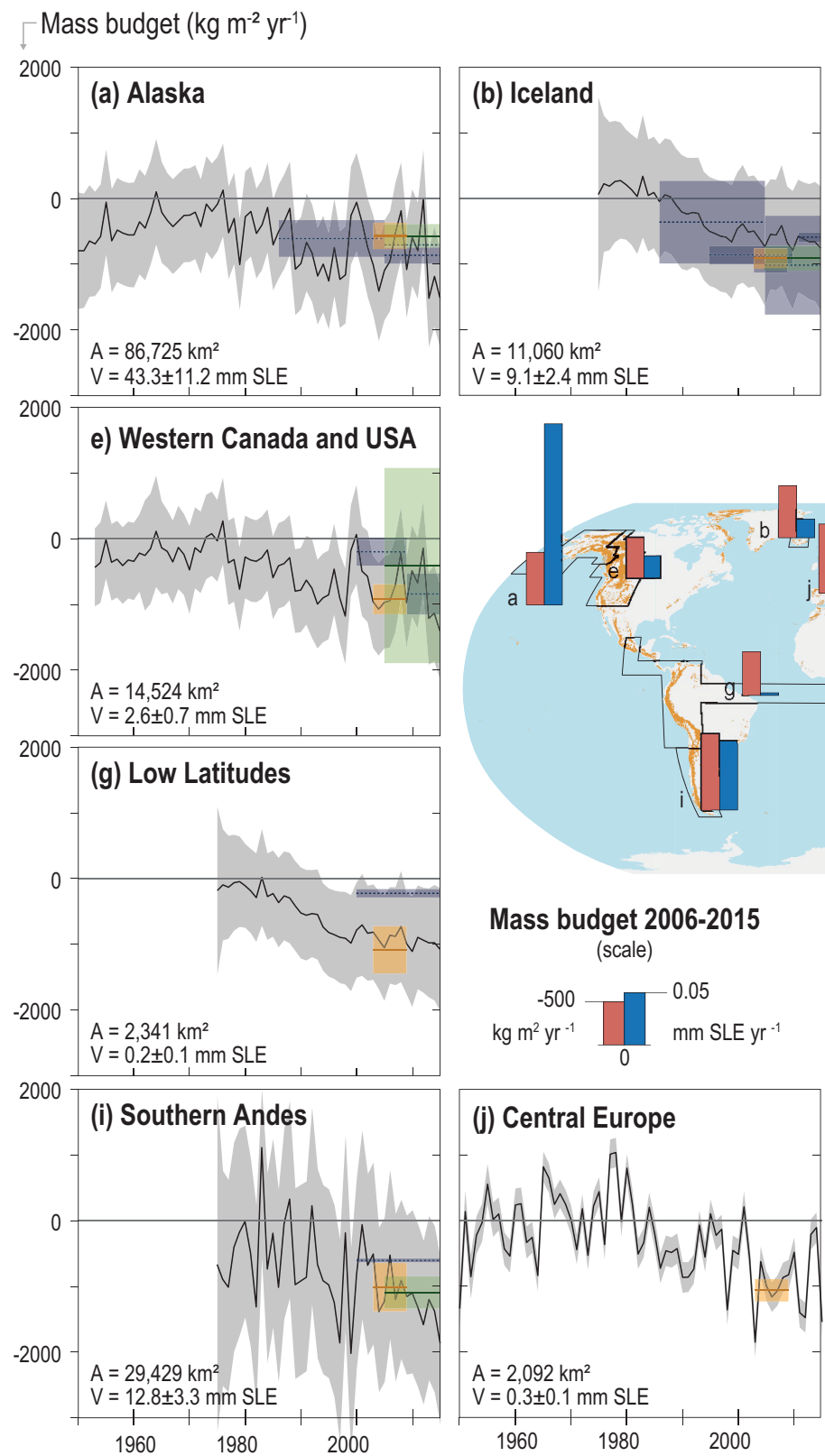

$\mathrm{V}=9.1 \pm 2.4 \mathrm{~mm}$ SLE
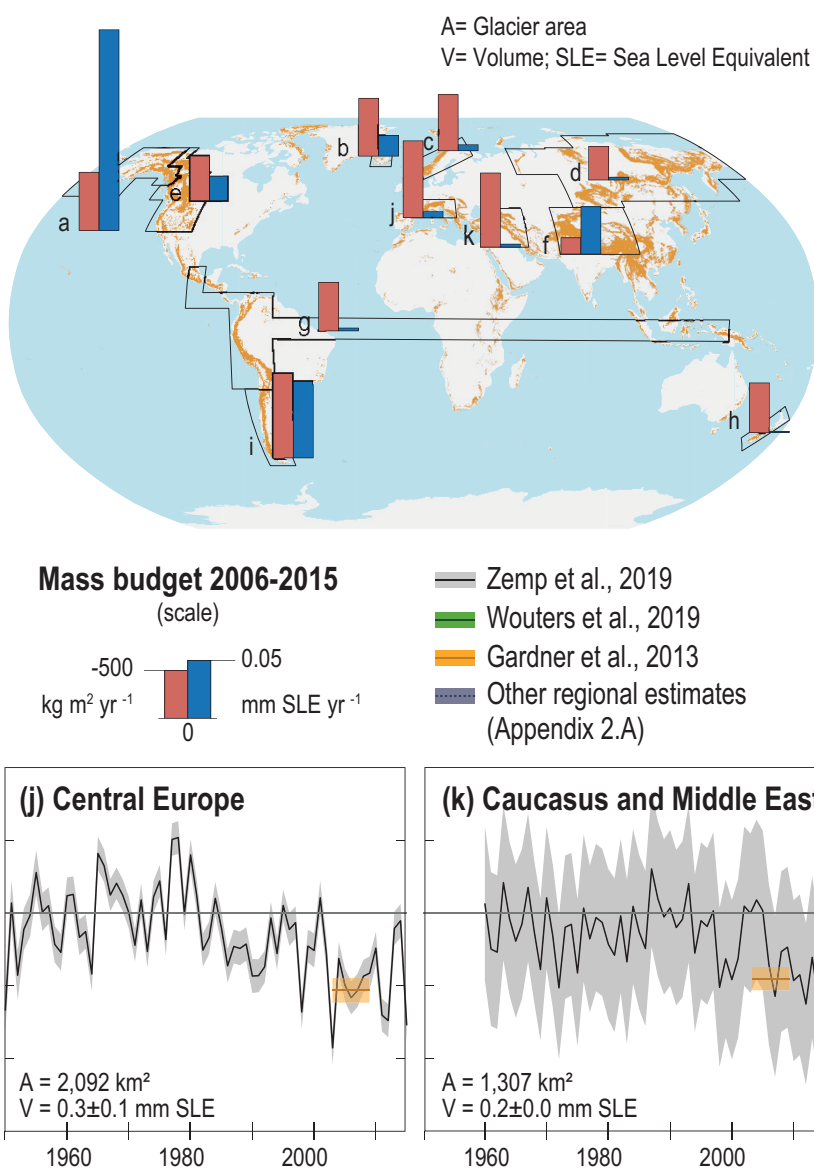

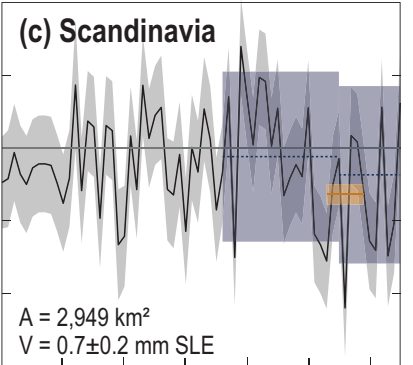

$\mathrm{A}=$ Glacier area
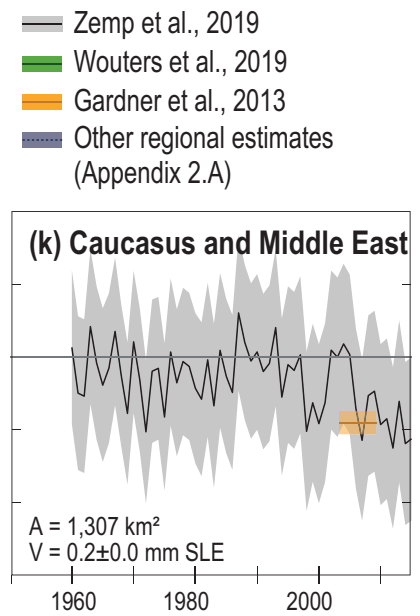

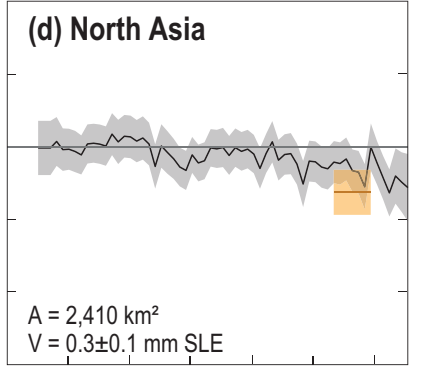

$\mathrm{V}=0.3 \pm 0.1 \mathrm{~mm} \mathrm{SLE}$
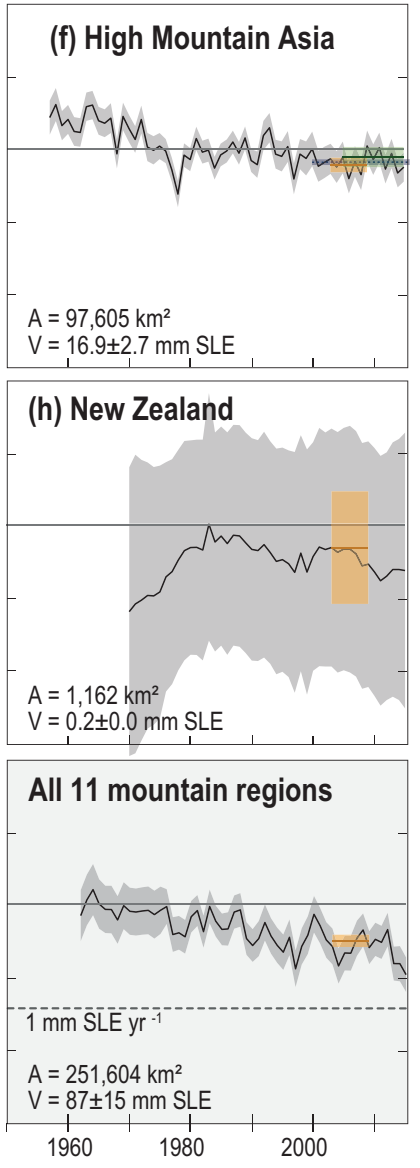

Figure 2.4 | Glacier mass budgets for the eleven mountain regions assessed in this Chapter (Figure 2.1) and these regions combined. Mass budgets for the remaining polar regions are shown in Chapter 3, Figure 3.8. Regional time series of annual mass change are based on glaciological and geodetic balances (Zemp et al., 2019). Superimposed are multi-year averages by Wouters et al. (2019) based on the Gravity Recovery and Climate Experiment (GRACE), only shown for the regions with glacier area $>3,000$ km². Estimates by Gardner et al. (2013) were used in the IPCC 5th Assessment Report (AR5). Additional regional estimates available in some regions and shown here are listed in Table 2.A.1. Annual and time-averaged mass-budget estimates include the errors reported in each study. Glacier areas (A) and volumes (V) are based on RGI Consortium (2017)

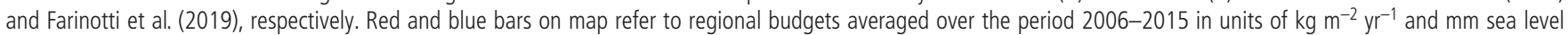
equivalent (SLE) $\mathrm{yr}^{-1}$, respectively, and are derived from each region's available mass-balance estimates (Appendix 2.A). 
(Solomina et al., 2016), this section focuses on observed glacier changes during recent decades and changes projected for the $21 \mathrm{st}$ century (Cross-Chapter Box 6 in Chapter 2).

Satellite and in situ observations of changes in glacier area, length and mass show a globally largely coherent picture of mountain glacier recession in the last decades (Zemp et al., 2015), although annual variability and regional differences are large (Figure 2.4; very high confidence). The global trend is statistically significant despite considerable interannual and regional variations (Medwedeff and Roe, 2017). Since AR5's global 2003-2009 estimate based on Gardner et al. (2013), several new estimates of global-scale glacier mass budgets have emerged using largely improved data coverage and methods (Bamber et al., 2018; Wouters et al., 2019; Zemp et al., 2019).

These estimates combined with available regional estimates (Table 2.A.1) indicate that the glacier mass budget of all mountain regions (excluding Antarctica, Greenland, the Canadian and Russian Arctic, and Svalbard) was very likely $-490 \pm 100 \mathrm{~kg} \mathrm{~m}^{-2} \mathrm{yr}^{-1}$ $\left(-123 \pm 24 \mathrm{Gt} \mathrm{yr}^{-1}\right)$ during the period 2006-2015 with most negative averages (less than $-850 \mathrm{~kg} \mathrm{~m}^{-2} \mathrm{yr}^{-1}$ ) in the Southern Andes, Caucasus/ Middle East, European Alps and Pyrenees. High Mountain Asia shows the least negative mass budget $\left(-150 \pm 110 \mathrm{~kg} \mathrm{~m}^{-2} \mathrm{yr}^{-1}\right.$, Figure 2.4), but variations within the region are large with most negative regional balance estimates in Nyainqentanglha, Tibet $\left(-620 \pm 230 \mathrm{~kg} \mathrm{~m}^{-2} \mathrm{yr}^{-1}\right)$ and slightly positive balances in the Kunlun Mountains for the period 2000-2016 (Brun et al., 2017). Due to large ice extent, the total mass loss and corresponding contribution to sea level 2006-2015 is largest in Alaska, followed by the Southern Andes and High Mountain Asia (Table 2.A.1). Zemp et al. (2019) estimated an increase in mean global-scale glacier mass loss by $\sim 30 \%$ between 1986-2005 and 2006-2015.

It is very likely that atmospheric warming is the primary driver for the global glacier recession (Marzeion et al., 2014; Vuille et al., 2018). There is limited evidence (high agreement) that human-induced increases in greenhouse gases have contributed to the observed mass changes (Hirabayashi et al., 2016). It was estimated that the anthropogenic fraction of mass loss of all glaciers outside Greenland and Antarctica increased from $25 \pm 35 \%$ during 1851-2010 to $69 \pm 24 \%$ during 1991-2010 (Marzeion et al., 2014).

Other factors, such as changes in meteorological variables other than air temperature or internal glacier dynamics, have modified the temperature-induced glacier response in some regions (high confidence). For example, glacier mass loss over the last seven decades on a glacier in the European Alps was intensified by higher air moisture leading to increased longwave irradiance and reduced sublimation (Thibert et al., 2018). Changes in air moisture have also been found to play a significant role in past glacier mass changes in eastern Africa (Prinz et al., 2016), while an increase in shortwave radiation due to reduced cloud cover contributed to an acceleration in glacier recession in the Caucasus (Toropov et al., 2019). In the Tien Shan mountains changes in atmospheric circulation in the North Atlantic and North Pacific in the 1970s resulted in an abrupt reduction in precipitation and thus snow accumulation, amplifying temperature-induced glacier mass loss (Duethmann et al., 2015).
Deposition of light absorbing particles, growth of algae and bacteria and local amplification phenomena such as the enhancement of particles concentration due to surface snow and ice melt, and cryoconite holes, have been shown to enhance ice melt (e.g., Ginot et al., 2014; Zhang et al., 2017; Williamson et al., 2019) but there is limited evidence and low agreement that long-term changes in glacier mass are linked to light absorbing particles (Painter et al., 2013; Sigl et al., 2018). Debris cover can modulate glacier melt but there is limited evidence on its role in recent glacier changes (Gardelle et al., 2012; Pellicciotti et al., 2015). Rapid retreat of calving outlet glaciers in Patagonia was attributed to changes in glacier dynamics (Sakakibara and Sugiyama, 2014).

Departing from this global trend of glacier recession, a small fraction of glaciers have gained mass or advanced in some regions mostly due to internal glacier dynamics or, in some cases, locally restricted climatic causes. For example, in Alaska 36 marine-terminating glaciers exhibited a complex pattern of periods of significant retreat and advance during 1948-2012, highly variable in time and lacking coherent regional behaviour (McNabb and Hock, 2014). These fluctuations can be explained by internal retreat-advance cycles typical of tidewater glaciers that are largely independent of climate (Brinkerhoff et al., 2017). Irregular and spatially inconsistent glacier advances, for example, in Alaska, Iceland and Karakoram, have been associated with surge-type flow instabilities largely independent of changes in climate (Sevestre and Benn, 2015; Bhambri et al., 2017; Section 2.3.2). Regional scale glacier mass gain and advances in Norway in the 1990s and in New Zealand between 1983-2008 have been linked to local increases in snow precipitation (Andreassen et al., 2005) and lower air temperatures (Mackintosh et al., 2017), respectively, caused by changes in atmospheric circulation. Advances of some glaciers in Alaska, the Andes, Kamchatka and the Caucasus were attributed to volcanic activity causing flow acceleration through enhanced melt water at the ice-bed interface (Barr et al., 2018).

Region averaged glacier mass budgets have been nearly balanced in the Karakoram since at least the 1970s (Bolch et al., 2017; Zhou et al., 2017; Azam et al., 2018), while slightly positive balances since 2000 have been reported in the western Kunlun Shan, eastern Pamir, and the central and northern Karakoram mountains (Gardelle et al., 2013; Brun et al., 2017; Lin et al., 2017; Berthier and Brun, 2019). This anomalous behavior has been related to specific mechanisms countering the effects of atmospheric warming, for example, an increase in cloudiness (Bashir et al., 2017) and snowfall (Kapnick et al., 2014) spatially heterogeneous glacier mass balance sensitivity (Sakai and Fujita, 2017), feedbacks due to intensified lowland irrigation (de Kok et al., 2018), and changes in summer atmospheric circulation (Forsythe et al., 2017).

There is medium evidence (high agreement) that recent glacier mass changes have modified glacier flow. A study covering all glaciers in High Mountain Asia showed glacier slowdown for regions with negative mass budgets since the 1970s and slightly accelerated glacier flow for Karakoram and West Kunlun regions where mass budgets were close to balance (Dehecq et al., 2019). Waechter et al. (2015) report reduced flow velocities in the St. Elias Mountains in North America, especially in areas of rapid ice thinning near glacier 
termini. In contrast Mouginot and Rignot (2015) found complex ice flow patterns with simultaneous acceleration and deceleration for glaciers of the Patagonian Icefield as well as large interannual variability during the last three decades concurrent with general thinning of the ice field.

\title{
Cross-Chapter Box 6 | Glacier Projections in Polar and High Mountain Regions
}

\author{
Authors: Regine Hock (USA), Andrew Mackintosh (Australia/New Zealand), Ben Marzeion (Germany)
}

Century-scale projections for all glaciers on Earth including those around the periphery of Greenland and Antarctica are presented here. Projections of the Greenland and Antarctic ice sheets are presented in Chapter 4. Future changes in glacier mass have global implications through their contribution to sea level change (Chapter 4) and local implication, for example, by affecting freshwater resources (Section 2.3.1). Glacier decline can also lead to loss of palaeoclimate information contained in glacier ice (Thompson et al., 2017).

AR5 included projections of 21st century glacier evolution from four process-based global-scale glacier models (Slangen and Van De Wal, 2011; Marzeion et al., 2012; Giesen and Oerlemans, 2013; Radić et al., 2014). Results have since been updated (Bliss et al., 2014; Slangen et al., 2017; Hock et al., 2019) using new glacier inventory data and/or climate projections, and projections from two additional models have been presented (Hirabayashi et al., 2013; Huss and Hock, 2015). These six models were driven by climate projections from 8-21 General Circulation Models (GCMs) from the CMIP5 (Taylor et al., 2012) forced by various RCPs, and results are systematically compared in Hock et al. (2019).

Based on these studies there is high confidence that glaciers in polar and high mountain regions will lose substantial mass by the end of the century. Results indicate global glacier mass losses by 2100 relative to 2015 of 18\% (likely range 11-25\%) (mean of all projections with range referring to \pm one standard deviation) for scenario RCP2.6 and 36\% (likely range 26-47\%) for RCP8.5, but relative mass reductions vary greatly between regions (Figure CB6.1). Projected end-of-century mean mass losses relative to 2015 tend to be largest in mountain regions dominated by smaller glaciers and relatively little ice cover, exceeding on average 80\%, for example, the European Alps, Pyrenees, Caucasus/Middle East, Low Latitudes and North Asia for RCP8.5 (see Figure 2.1 for region definitions). While these glaciers' contribution to sea level is negligible their large relative mass losses have implications for streamflow (Section 2.3.1, FAQ 2.1).

The magnitude and timing of these projected mass losses is assigned medium confidence because the projections have been carried out using relatively simple models calibrated with limited observations in some regions and diverging initial glacier volumes. For example, mass loss by iceberg calving and subaqueous melt processes that can be particularly important components of glacier mass budgets in polar regions (McNabb et al., 2015) have only been included in one global-scale study (Huss and Hock, 2015). In addition instability mechanisms that can cause rapid glacier retreat and mass loss are not considered (Dunse et al., 2015; Sevestre et al., 2018; Willis et al., 2018).

The projected global-scale relative mass losses 2015-2100 correspond to a sea level contribution of 94 (likely range 69-119) $\mathrm{mm}$ sea level equivalent (SLE) corresponding to an average rate of 1.1 (likely range 0.8-1.4) mm SLE yr ${ }^{-1}$ for RCP2.6, and 200 (likely range

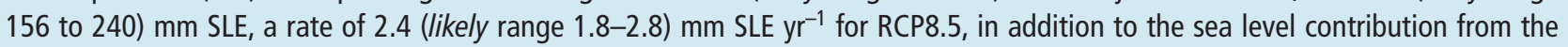
Greenland and Antarctic ice sheets (Chapter 4). Averages refer to the mean and ranges to \pm one standard deviation of all simulations. For RCP2.6, rates increase only slightly until approximately year 2040 with a steady decline thereafter, as glaciers retreat to higher elevations and reach new equilibrium. In contrast, for RCP8.5, the sea level contribution from glaciers increases steadily for most of the century, reaching an average maximum rate exceeding $3 \mathrm{~mm} \mathrm{SLE} \mathrm{yr}{ }^{-1}$ (Hock et al., 2019). For both RCPs the polar regions are the largest contributors with projected mass reductions by 2100 relative to 2015 combined for the Antarctic periphery, Arctic Canada, the Greenland periphery, Iceland, Russian Arctic, Scandinavia and Svalbard ranging from 16\% (likely range 9 to 23\%) for RCP2.6 to 33\% (likely range 22 to $44 \%$ ) for RCP8.5. Due to extensive ice cover, these regions make up roughly $80 \%$ of the global sea level contribution from glaciers by 2100. The global projections are similar to those reported in AR5 for the period 2081-2100 relative to 1986-2005, if differences in period length and domain are accounted for (AR5's glacier estimates excluded the Antarctic periphery). The eleven mountain regions covered in Chapter 2 are likely to lose 22-44\% of their glacier mass by 2100 relative to 2015 for RCP2.6 and $37-57 \%$ for RCP8.5. Worldwide many glaciers are expected to disappear by 2100 regardless emission scenario, especially in regions with smaller glaciers (very high confidence) (Cullen et al. 2013; Rabatel et al., 2013; Huss and Fischer, 2016; Rabatel et al., 2017). 
Cross-Chapter Box 6 (continued)

The global-scale projections (Figure CB6.1) are consistent with results from regional-scale studies using more sophisticated models. Kraaijenbrink et al. (2017) projected mass losses for all glaciers in High Mountain Asia of $64 \pm 5 \%$ (RCP8.5) by the end of the century (2071-2100) compared to 1996-2015. A high-resolution regional glaciation model including ice dynamics indicated that by 2100 glacier volume in western Canada will shrink by 70\% (RCP2.6) to 90\% (RCP8.5) relative to 2005 (Clarke et al., 2015). Zekollari et al. (2019) projected that the glaciers in the European Alps will largely disappear by 2100 (94 \pm 4\% mass loss relative to 2017) for RCP8.5, while projected mass losses are $63 \pm 11 \%$ for RCP2.6.

AR5 concluded with high confidence that due to a pronounced imbalance between current glacier mass and climate, glaciers are expected to further recede even in the absence of further climate change. Studies since AR5 agree and provide further evidence (Mernild et al., 2013; Marzeion et al., 2018).

$$
\text { Mass (\%) }
$$

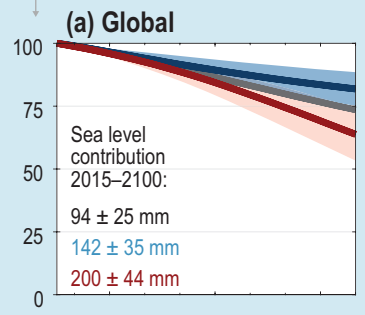

(b) Global excl. A+G

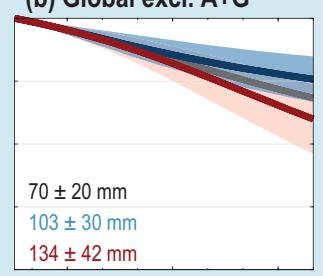

(g) Russian Arctic
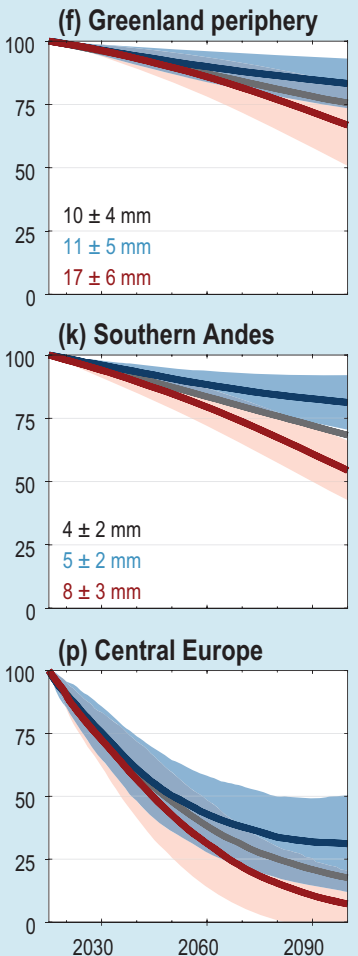

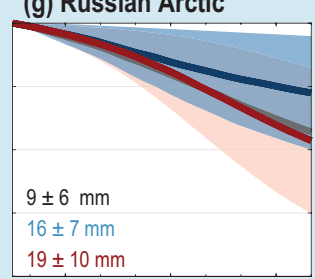

\section{(I) Iceland}

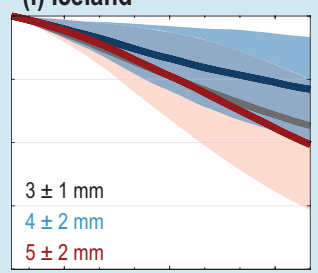

(q) Low Latitudes

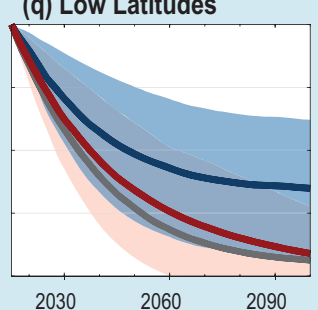

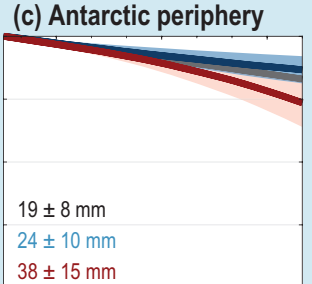

(h) Arctic Canada $\mathrm{S}$

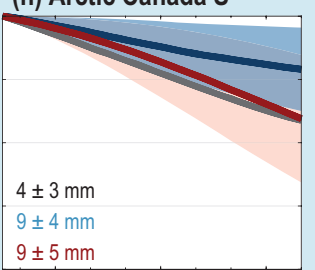

(m) W Canada \& USA

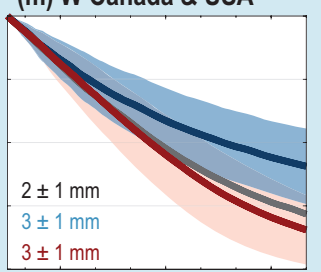

(r) New Zealand

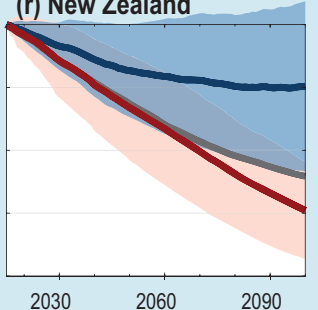

(d) Arctic Canada N
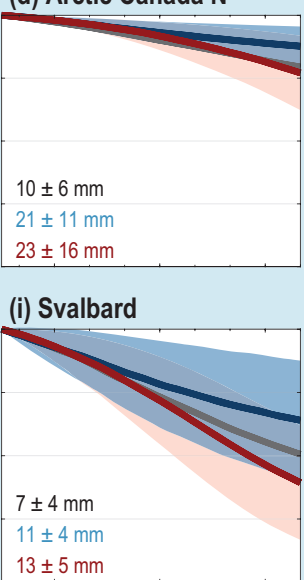

(n) Scandinavia

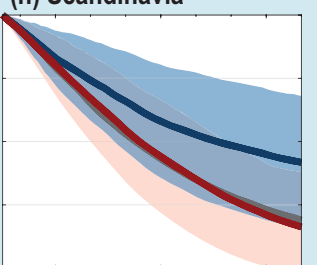

(s) Caucasus/Middle East

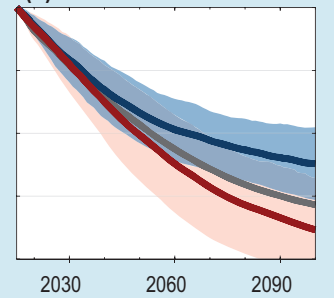

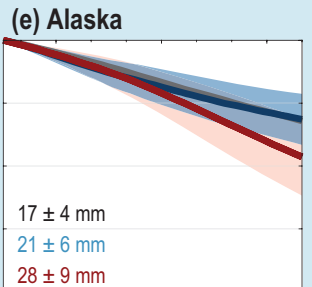

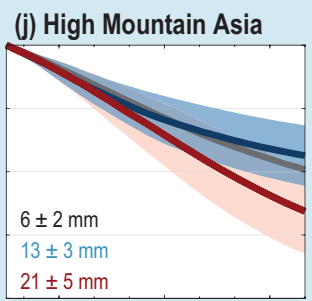

(o) North Asia

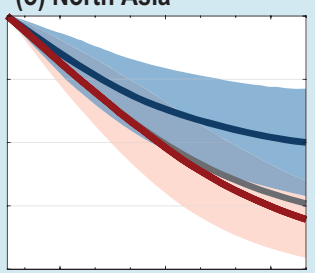

Scenario

(Number of runs, number of models)

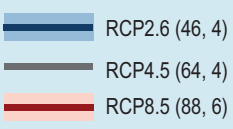

Figure CB6.1 | Projected glacier mass evolution between 2015 and 2100 relative to each region's glacier mass in 2015 (100\%) based on three Representative Concentration Pathways (RCP) emission scenarios (Cross-Chapter Box 1 in Chapter 1). Thick lines show the averages of 46 to 88 model projections based on four to six glacier models for the same RCP, and the shading marks \pm 1 standard deviation (not shown for RCP4.5 for better readability). Global projections are shown excluding and including the Antarctic (A) and Greenland (G) periphery. Regional sea level contributions are given for three RCPs for all regions with $>0.5 \mathrm{~mm}$ sea level equivalent (SLE) between 2015-2100. The Low Latitudes region includes the glaciers in the tropical Andes, Mexico, eastern Africa and Indonesia. Region Alaska includes adjacent glaciers in the Yukon and British Columbia, Canada. Regions are sorted by glacier volume according to Farinotti et al. (2019). Data based on Marzeion et al. (2012); Giesen and Oerlemans (2013); Hirabayashi et al. (2013); Bliss et al. (2014); Huss and Hock (2015); Slangen et al. (2017). Modified from Hock et al. (2019). 


\subsubsection{Permafrost}

This section assesses permafrost, but not seasonally frozen ground, in high mountain areas. As mountains also exist in polar areas, some overlap exists between this section and Chapter 3. Observations of permafrost are scarce (Tables 2.1 and 2.2, PERMOS, 2016; Bolch et al., 2018) and unevenly distributed among and within mountain regions. Unlike glaciers and snow, permafrost is a subsurface phenomenon that cannot easily be observed remotely. As a consequence, its distribution and change are less understood than for glaciers or snow, and in many mountain regions it can only be inferred (Gruber et al., 2017). Permafrost thaw and degradation impact people via runoff and water quality (Section 2.3.1), hazards and infrastructure (Section 2.3.2) and greenhouse gas emissions (Box 2.2).

AR5 and IPCC's Special Report on 'Managing the Risks of Extreme Events and Disasters to Advance Climate Change Adaptation' (SREX) assessed permafrost change globally, but not separately for mountains. AR5 concluded that permafrost temperatures had increased in most regions since the early 1980s (high confidence), although warming rates varied regionally, and attributed this warming to increased air temperature and changes in snow cover (high confidence). The temperature increase for colder permafrost was generally greater than for warmer permafrost (high confidence). SREX found a likely warming of permafrost in recent decades and expressed high confidence that its temperatures will continue to increase. AR5 found decreases of northern high-latitude near surface permafrost for 2016-2035 to be very likely and a general retreat of permafrost extent for the end of the 21st century and beyond to be virtually certain. While some permafrost phenomena, methods of observation and scale issues in scenario simulations are specific to mountainous terrain, the basic mechanisms connecting climate and permafrost are the same in mountains and polar regions.

Between 3.6-5.2 million $\mathrm{km}^{2}$ are underlain by permafrost in the eleven high mountain regions outlined in Figure 2.1 (medium confidence) based on data from two modelling studies (Gruber, 2012; Obu et al., 2019). For comparison, this is 14-21 times the area of glaciers (Section 2.2.3) in these regions (Figure 2.1) or $27-29 \%$ of the global permafrost area. The distribution of permafrost in mountains is spatially highly heterogeneous, as shown in detailed regional modelling studies (Boeckli et al., 2012; Bonnaventure et al., 2012; Westermann et al., 2015; Azócar et al., 2017; Zou et al., 2017).

Permafrost in the European Alps, Scandinavia, Canada, Mongolia, the Tien Shan and the Tibetan Plateau has warmed during recent decades and some observations reveal ground-ice loss and permafrost degradation (high confidence). The heterogeneity of mountain environments and scarcity of long-term observations challenge the quantification of representative regional or global warming rates. A recent analysis finds that permafrost at 28 mountain locations in the European Alps, Scandinavia, Canada as well as High Mountain Asia and North Asia, warmed on average by $0.19 \pm 0.05^{\circ} \mathrm{C}$ per decade between 2007-2016 (Biskaborn et al., 2019). Over longer periods, observations in the European Alps, Scandinavia, Mongolia, the Tien Shan and the Tibetan Plateau (see also Cao et al., 2018) show general warming (Table 2.1, Figure 2.5) and degradation of permafrost at individual sites (e.g., Phillips et al., 2009). Permafrost close to $0^{\circ} \mathrm{C}$ warms at a lower rate than colder permafrost because ground-ice melt slows warming. Similarly, bedrock warms faster than debris or soil because of low ice content. For example, several European bedrock sites (Table 2.1) have warmed rapidly, by up to $1^{\circ} \mathrm{C}$ per decade, during the past two decades. By contrast, total warming of $0.5^{\circ} \mathrm{C}-0.8^{\circ} \mathrm{C}$ has been inferred for the second half of the 20th century based on thermal gradients at depth in an ensemble of European bedrock sites (Isaksen et al., 2001; Harris et al., 2003). Warming has been shown to accelerate at sites in Scandinavia (Isaksen et al., 2007) and in mountains globally within the past decade (Biskaborn et al., 2019). During recent decades, rates of permafrost warming in the European Alps and Scandinavia exceeded values of the late 20th century (limited evidence, high agreement).

The observed thickness of the active layer (see Annex I: Glossary), the layer of ground above permafrost subject to annual thawing and freezing, increased in the European Alps, Scandinavia (Christiansen et al., 2010), and on the Tibetan Plateau during the past few decades (Table 2.2), indicating permafrost degradation. Geophysical monitoring in the European Alps during approximately the past 15 years revealed increasing subsurface liquid water content (Hilbich et al., 2008; Bodin et al., 2009; PERMOS, 2016), indicating gradual ground-ice loss.

During recent decades, the velocity of rock glaciers in the European Alps exceeded values of the late 20th century (limited evidence, high agreement). Some rock glaciers, that is, masses of ice-rich debris that show evidence of past or present movement, show increasing velocity as a transient response to warming and water input, although continued permafrost degradation would eventually inactivate them (Ikeda and Matsuoka, 2002). Rock glacier velocities observed in the European Alps in the 1990s were on the order of a few decimetres per year and during approximately the past 15 years they often were about 2-10 times higher (Bodin et al., 2009; Lugon and Stoffel, 2010; PERMOS, 2016). Destabilisation, including collapse and rapid acceleration, has been documented (Delaloye et al., 2010; Buchli et al., 2013; Bodin et al., 2016). One particularly long time series shows velocities around 1960 just slightly lower than during recent years (Hartl et al., 2016). In contrast to nearby glaciers, no clear change in rock glacier velocity or elevation was detected at a site in the Andes between 1955-1996 (Bodin et al., 2010). The majority of similar landforms investigated in the Alaska Brooks Range increased their velocity since the 1950s, while few others slowed down (Darrow et al., 2016).

Decadal-scale permafrost warming and degradation are driven by air temperature increase and additionally affected by changes in snow cover, vegetation and soil moisture. Bedrock locations, especially when steep and free of snow, produce the most direct signal of climate change on the ground thermal regime (Smith and Riseborough, 1996), increasing the confidence in attribution. Periods of cooling, one or few years long, have been observed and attributed to extraordinary low-snow conditions (PERMOS, 2016). Extreme increases of active-layer thickness often correspond with summer heat waves (PERMOS, 2016) and permafrost degradation can be accelerated by water percolation (Luethi et al., 2017). Similarity and 
synchronicity of interannual to decadal velocity changes of rock glaciers within the European Alps (Bodin et al., 2009; Delaloye et al., 2010) and the Tien Shan (Sorg et al., 2015), suggest common regional forcing such as summer air temperature or snow cover.

Because air temperature is the major driver of permafrost change, permafrost in high mountain regions is expected to undergo increasing thaw and degradation during the 21st century, with stronger consequences expected for higher greenhouse gas emission scenarios (very high confidence). Scenario simulations for the Tibetan Plateau until 2100 estimate permafrost area to be strongly reduced, for example by 22-64\% for RCP2.6 and RCP8.5 and a spatial resolution of $0.5^{\circ}$ (Lu et al., 2017). Such coarse-scale studies (Guo et al., 2012; Slater and Lawrence, 2013; Guo and Wang, 2016), however, are of limited use in quantifying changes and informing impact studies in steep terrain due to inadequate representation of topography (Fiddes and Gruber, 2012). Fine-scale simulations, on the other hand, are local or regional, limited in areal extent and differ widely in their representation of climate change and permafrost. They reveal regional and elevational differences of warming and degradation (Bonnaventure and Lewkowicz, 2011; Hipp et al., 2012; Farbrot et al., 2013) as well as warming rates that differ between locations (Marmy et al., 2016) and seasons (Marmy et al., 2013). While structural differences in simulations preclude a quantitative summary, these studies agree on increasing warming and thaw of permafrost for the 21st century and reveal increased loss of permafrost under stronger atmospheric warming (Chadburn et al., 2017). Permafrost thaw at depth is slow but can be accelerated by mountain peaks warming from multiple sides (Noetzli and Gruber, 2009) and deep percolation of water (Hasler et al., 2011). Near Mont Blanc in the European Alps, narrow peaks below 3,850 m a.s.l. may lose permafrost entirely under RCP8.5 by the end of the 21st century (Magnin et al., 2017). As ground-ice from permafrost usually melts slower than glacier ice, some mountain regions will transition from having abundant glaciers to having few and small glaciers but large areas of permafrost that is thawing (Haeberli et al., 2017).

Table 2.1 Observed changes in permafrost mean annual ground temperature (MAGT) in mountain regions. Values are based on individual boreholes or ensembles of several boreholes. The MAGT refers to the last year in a period and is taken from a depth of 10-20 m unless the borehole is shallower. Region names refer to Figure 2.1. Numbers in brackets indicate how many sites are summarised for a particular surface type and area; the underscored value is an average. Elevation is metres above sea level (m a.s.l.).

\begin{tabular}{|c|c|c|c|c|c|c|}
\hline Region & $\begin{array}{l}\text { Elevation } \\
\text { [m a.s.l.] }\end{array}$ & Surface Type & Period & $\begin{array}{l}\mathrm{MAGT} \\
{\left[{ }^{\circ} \mathrm{C}\right]}\end{array}$ & $\begin{array}{l}\text { MAGT trend } \\
{\left[{ }^{\circ} \mathrm{C} \text { per decade] }\right.}\end{array}$ & Reference \\
\hline Global & $>1,000$ & various (28) & $2006-2017$ & not specified & $0.2 \pm 0.05$ & Biskaborn et al. (2019) \\
\hline \multirow[b]{2}{*}{ European Alps } & $2,500-3,000$ & $\begin{array}{l}\text { debris or coarse blocks } \\
(>10)\end{array}$ & $\begin{array}{l}1987-2005 \\
2006-2017\end{array}$ & $\begin{array}{l}>-3 \\
>-3\end{array}$ & $\begin{array}{l}0.0-0.2 \\
0.0-0.6\end{array}$ & $\begin{array}{l}\text { PERMOS (2016) } \\
\text { Noetzli et al. (2018) }\end{array}$ \\
\hline & $3,500-4,000$ & bedrock (4) & 2008-2017 & $>-5.5$ & $0.0-1.0$ & $\begin{array}{l}\text { Pogliotti et al. (2015) } \\
\text { Magnin et al. (2015) } \\
\text { Noetzli et al. (2018) }\end{array}$ \\
\hline \multirow[b]{2}{*}{ Scandinavia } & $1,402-1,505$ & moraine (3) & 1999-2009 & 0 to -0.5 & $0.0-0.2$ & Isaksen et al. (2011) \\
\hline & $1,500-1,894$ & bedrock (2) & 1999-2009 & -2.7 & 0.5 & $\begin{array}{l}\text { Christiansen et al. } \\
\text { (2010) }\end{array}$ \\
\hline \multirow{2}{*}{$\begin{array}{l}\text { High Mountain Asia } \\
\text { (Tien Shan) }\end{array}$} & $\sim 3,330$ & bare soil (2) & $1974-2009$ & -0.5 to -0.1 & $0.3-0.6$ & Zhao et al. (2010) \\
\hline & 3,500 & meadow (1) & $1992-2011$ & -1.1 & 0.4 & Liu et al. (2017) \\
\hline \multirow{4}{*}{$\begin{array}{l}\text { High Mountain Asia } \\
\text { (Tibetan Plateau) }\end{array}$} & $\sim 4,650$ & meadow (6) & $2002-2012$ & -1.52 to -0.41 & $0.08-0.24$ & Wu et al. (2015) \\
\hline & $\sim 4,650$ & steppe (3) & $2002-2012$ & -0.79 to -0.17 & & Wu et al. (2015) \\
\hline & $\sim 4,650$ & bare soil (1) & 2003-2012 & -0.22 & 0.15 & Wu et al. (2015) \\
\hline & $4,500-5,000$ & unknown (6) & 2002-2011 & -1.5 to -0.16 & $0.08-0.24$ & Peng et al. (2015) \\
\hline North Asia (Mongolia) & $1,350-2,050$ & steppe (6) & $2000-2009$ & -0.06 to -1.54 & $0.2-0.3$ & Zhao et al. (2010) \\
\hline
\end{tabular}

Table 2.2 Observed changes of active-layer thickness (ALT) in mountain regions. Numbers in brackets indicate how many sites are summarised for a particular surface type and area. Region names refer to Figure 2.1. Elevation is metres above sea level ( $m$ a.s.l.).

\begin{tabular}{|c|c|c|c|c|c|c|}
\hline Region & $\begin{array}{l}\text { Elevation } \\
\text { [m a.s.l.] }\end{array}$ & $\begin{array}{l}\text { Surface } \\
\text { Type }\end{array}$ & Period & $\begin{array}{c}\text { ALT in last year } \\
{[\mathrm{m}]}\end{array}$ & $\begin{array}{c}\text { ALT trend } \\
\text { [cm per decade] }\end{array}$ & Reference \\
\hline Scandinavia & $353-507$ & peatland (9) & $\begin{array}{l}1978-2006 \\
1997-2006\end{array}$ & $\sim 0.65-0.85$ & $\begin{array}{r}7-13 \\
13-20 \\
\end{array}$ & $\begin{array}{l}\text { Åkerman and } \\
\text { Johansson (2008) }\end{array}$ \\
\hline European Alps & $2,500-2,910$ & bedrock (4) & 2000-2014 & $4.2-5.2$ & $10-100$ & PERMOS (2016) \\
\hline $\begin{array}{l}\text { High Mountain Asia } \\
\text { (Tien Shan) }\end{array}$ & 3,500 & meadow (1) & $1992-2011$ & 1.70 & 19 & Liu et al. (2017) \\
\hline \multirow{4}{*}{$\begin{array}{l}\text { High Mountain Asia } \\
\text { (Tibetan Plateau) }\end{array}$} & $4,629-4,665$ & meadow (6) & $2002-2012$ & $2.11-2.3$ & $34.8-45.7$ & Wu et al. (2015 \\
\hline & $4,638-4,645$ & steppe (3) & $2002-2012$ & $2.54-3.03$ & $39.6-67.2$ & Wu et al. (2015) \\
\hline & 4,635 & bare soil (1) & $2002-2012$ & 3.38 & 18.9 & Wu et al. (2015) \\
\hline & 4,848 & meadow & 2006-2014 & $1.92-2.72$ & $15.2-54$ & Lin et al. (2016) \\
\hline
\end{tabular}




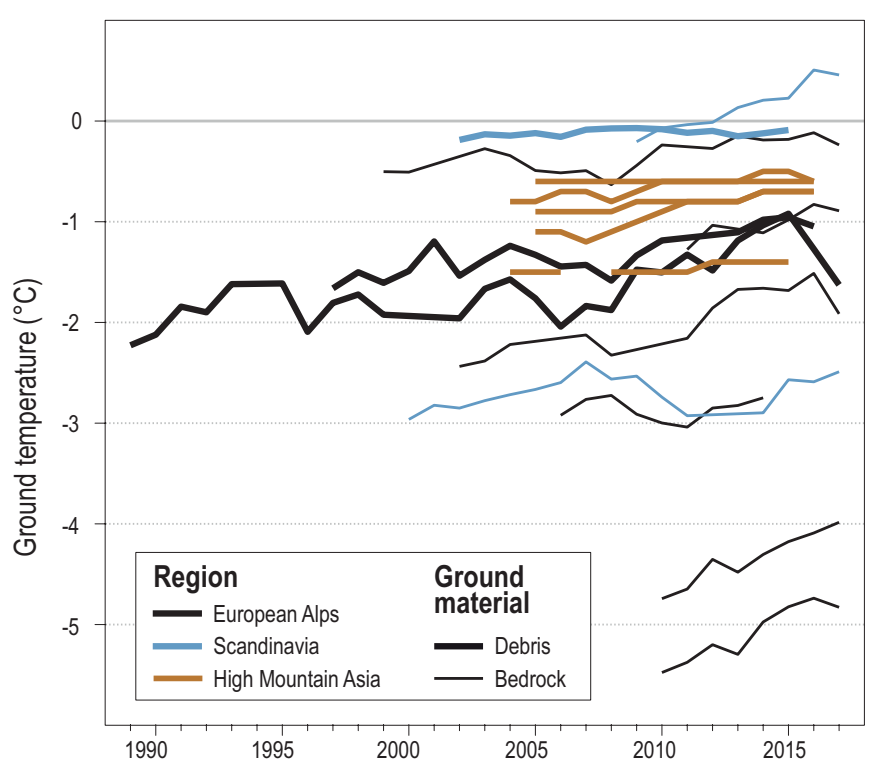

Figure 2.5 Mean annual ground temperature from boreholes in debris and bedrock in the European Alps, Scandinavia and High Mountain Asia. Temperatures differ between locations and warming trends can be interspersed by short periods of cooling. One location shows degrading of permafrost. Overall, the number of observed boreholes is small and most records are short. The depth of measurements is approximately $10 \mathrm{~m}$, and years without sufficient data are omitted (Noetzli et al., 2018).

\subsubsection{Lake and River Ice}

Based on limited evidence, AR5 reported shorter seasonal ice cover duration during the past decades (low confidence), however, did not specifically address changes in mountain lakes and rivers. Observations of extent, timing, duration and thickness of lake and river ice rely mostly on in situ measurements (e.g., Sharma et al., 2019) and, increasingly on remote sensing (Duguay et al., 2014). Lake and river ice studies focusing specifically on mountain regions are rare but observations from lakes in the European Alps, Scandinavia and the Tibetan Plateau show highly variable trends in ice cover duration during the past decades.

For example, Cai et al. (2019) reported shorter ice cover duration for 40 lakes and longer duration for 18 lakes on the Tibetan Plateau during the period 2000-2017. Similarly, using microwave remote sensing, Du et al. (2017) found shorter ice cover duration for 43 out of 71 lakes $>50 \mathrm{~km}^{2}$ including lakes on the Tibetan Plateau during $2002-2015$, but only five of these had statistically significant trends $(p<0.05)$, due to large interannual variability. The variable trends in the duration of lake ice cover on the Tibetan Plateau between 20022015 corresponded to variable trends in surface water temperatures. of 52 study lakes in this region, 31 lakes showed a mean warming rate of $0.055 \pm 0.033^{\circ} \mathrm{C} \mathrm{yr}^{-1}$, and 21 lakes showed a mean cooling rate of $-0.053 \pm 0.038^{\circ} \mathrm{C} \mathrm{yr}^{-1}$ during 2001-2012 (Zhang et al., 2014). Kainz et al. (2017) reported a significant $(p<0.05)$ increase in the interannual variability in ice cover duration for a subalpine lake in Austria during 1921-2015 in addition to a significant trend in later freeze on, earlier ice break up and shorter ice cover duration.
A significant $(p<0.05)$ trend towards shorter ice cover duration was found for another Austrian alpine lake during 1972-2015 (Niedrist et al., 2018).

Highly variable trends were also found in the timing and magnitude of river ice jams during 1903-2015, as reported by Rokaya et al. (2018) for Canadian rivers, including rivers in the mountains. Most of the variability in river ice trends could be explained by variable water flow, in particular due to flow regulation.

There is high confidence that air temperature and solar radiation are the most important drivers to explain observed changes of lake ice dynamics (Sharma et al., 2019). In mountainous regions where the interannual variability in ice cover duration is high, additional drivers become important, for example, morphometry, wind exposure, salinity, and hydrology, in particular hydrological processes driven by glaciers (Kropácek et al., 2013; Song et al., 2014; Yao et al., 2016; Gou et al., 2017). Despite high spatial and temporal variability in lake and river ice cover dynamics in mountain regions there is limited evidence (high agreement) that further air temperature increases will result in a general trend towards later freezing, earlier break-up, and shorter ice cover duration in the future (Gebre et al., 2014; Du et al., 2017).

Overall, there is only limited evidence on changes in lake and river ice specifically in the mountains, indicating a trend, but not universally, towards shorter lake ice cover duration consistent with increased water temperature. 


\section{Box 2.2 Local, Regional and Global Climate Feedbacks Involving the Mountain Cryosphere}

The cryosphere interacts with the environment and contributes to several climate feedbacks, most notably ones involving the snow cover, referred to as the snow albedo feedback. The presence or absence of snow on the ground drives profound changes in the energy budget of land surfaces, hence influencing the physical state of the overlying atmosphere (Armstrong and Brun, 2008). The reduction of snow on the ground, potentially amplified by aerosol deposition and modulated by interactions with the vegetation, increases the absorption of incoming solar radiation and leads to atmospheric warming. In mountain regions, this positive feedback loop mostly operates at the local scale and is seasonally variable, with most visible effects at the beginning and end of the snow season (Scherrer et al., 2012). Examples of other mechanisms contributing to local feedbacks are introduced in Box 2.1. At the regional scale, feedbacks associated with deposition of light absorbing particles and enhanced snow albedo feedback were shown to induce surface air warming (locally up to $2^{\circ} \mathrm{C}$ ) (Ménégoz et al., 2014) with accelerated snow cover reduction (Ji, 2016; Xu et al., 2016), and may also influence the Asian monsoon system (Yasunari et al., 2015). However, many of these studies have considered so-called rapid adjustments, without changes in large-scale atmospheric circulation patterns, because they used regional or global models constrained by large-scale synoptic fields. In summary, regional climate feedbacks involving the high mountain cryosphere, particularly the snow albedo feedback, have only been detected in large mountain regions such as the Himalaya, using global and regional climate models (medium confidence).

Global-scale climate feedbacks from the cryosphere remain largely unexplored with respect to the proportion originating from high mountains. Although mountain topography affects global climate (e.g., Naiman et al., 2017), there is little evidence for mountain-cryosphere specific feedbacks, largely because of the limited spatial extent of the mountain cryosphere. The most relevant feedback probably relates to permafrost in mountains, which contain about $28 \%$ of the global permafrost area (Section 2.2.4). Organic carbon stored in permafrost can be decayed following thaw and transferred to the atmosphere as carbon dioxide or methane (Schuur et al., 2015). This self-reinforcing effect accelerates the pace of climate change and operates in polar (Section 3.4.1.2.3) and mountain areas alike (Mu et al., 2017; Sun et al., 2018a). In contrast to polar areas, however, there is limited evidence and low agreement on the total amount of permafrost carbon in mountains because of differences in upscaling and difficulties to distinguish permafrost and seasonally frozen soils due to the lack of data. For example, on the Tibetan Plateau, the top $3 \mathrm{~m}$ of permafrost are estimated to contain about 15 petagrams (Ding et al., 2016) and mountain soils with permafrost globally are estimated to contain approximately 66 petagrams of organic carbon (Bockheim and Munroe, 2014). At the same time, there is limited evidence and high agreement that the average density $\left(\mathrm{kg} \mathrm{C} \mathrm{m}^{-2}\right)$ of permafrost carbon in mountains is lower than in other areas. For example, densities of soil organic carbon are low in the sub-arctic Ural (Dymov et al., 2015) and 1-2 orders of magnitude lower in subarctic Sweden (Fuchs et al., 2015) in comparison to lowland permafrost, and 50\% lower in mountains than in steppe-tundra in Siberia and Alaska (Zimov et al., 2006). Some mechanisms of soil carbon decay and transfer to the atmosphere in mountains are similar to those in lowland areas, for example collapse following thaw in peatlands (Mu et al., 2016; Mamet et al., 2017), and some are specific to areas with steep slopes, for example drainage of water from thawing permafrost leading to soil aeration (Dymov et al., 2015). There is no global-scale analysis of the climate feedback from permafrost in mountains. Given that projections indicate increasing thaw and degradation of permafrost in mountains during the 21 st century (very high confidence) (Section 2.2.4), a corresponding increase in greenhouse gas emissions can be anticipated but is not quantified.

\subsection{Mountain Social-Ecological Systems: Impacts, Risks and Human Responses}

\subsubsection{Water Resources}

The mountain cryosphere is an important source of freshwater in the mountains themselves and in downstream regions. The runoff per unit area generated in mountains is on average approximately twice as high as in lowlands (Viviroli et al., 2011) making mountains a significant source of fresh water in sustaining ecosystem and supporting livelihoods in and far beyond the mountain ranges themselves. The presence of snow, glaciers, and permafrost generally exert a strong control on the amount, timing and biogeochemical properties of runoff (FAQ 2.1). Changes to the cryosphere due to climate change can alter freshwater availability with direct consequences for human populations and ecosystems.

\subsubsection{Changes in River Runoff}

AR5 reported increased winter flows and a shift in timing towards earlier spring snowmelt runoff peaks during previous decades (robust evidence, high agreement). In glacier-fed river basins, it was projected that melt water yields from glaciers will increase for decades in many regions but then decline (very high confidence). These findings have been further supported and refined by a wealth of new studies since AR5.

Recent studies indicate considerable changes in the seasonality of runoff in snow and glacier dominated river basins (very high confidence; Table SM2.9). Several studies have reported an increase in average winter runoff over the past decades, for example in Western Canada (Moyer et al., 2016), the European Alps (Bocchiola, 2014; Bard et al., 2015) and Norway (Fleming and Dahlke, 2014), 
due to more precipitation falling as rain under warmer conditions. Summer runoff has been observed to decrease in basins, for example in Western Canada (Brahney et al., 2017) and the European Alps (Bocchiola, 2014), but to increase in several basins in High Mountain Asia (Mukhopadhyay and Khan, 2014; Duethmann et al., 2015; Reggiani and Rientjes, 2015; Engelhardt et al., 2017). Both increases, for example, in Alaska (Beamer et al., 2016) and the Tien Shan (Wang et al., 2015; Chen et al., 2016), and decreases, for example, in Western Canada (Brahney et al., 2017) have also been found for average annual runoff. In Western Austria, Kormann et al. (2015) detected an increase in annual flow at high elevations and a decrease at low elevations between 1980-2010.

These contrasting trends for summer and annual runoff often result from spatially variable changes in the contribution of glacier and snow melt. As glaciers shrink, annual glacier runoff typically first increases, until a turning point, often called 'peak water' is reached, upon which runoff declines (FAQ 2.1). There is robust evidence and high agreement that peak water in glacier-fed rivers has already passed with annual runoff declining especially in mountain regions with predominantly smaller glaciers, for example, in the tropical Andes (Frans et al., 2015; Polk et al., 2017), Western Canada (Fleming and Dahlke, 2014; Brahney et al., 2017) and the Swiss Alps (Huss and Fischer, 2016). A global modelling study (Huss and Hock, 2018) suggests that peak water has been reached before 2019 for $82-95 \%$ of the glacier area in the tropical Andes, $40-49 \%$ in Western Canada and USA, and $55-67 \%$ in Central Europe (including European Alps and Pyrenees) and the Caucasus (Figure 2.6).

Projections indicate a continued increase in winter runoff in many snow and/or glacier-fed rivers over the 21 st century (high confidence) regardless of the climate scenario, for example, in North America (Schnorbus et al., 2014; Sultana and Choi, 2018), the European Alps (Addor et al., 2014; Bosshard et al., 2014), Scotland (Capell et al., 2014) and High Mountain Asia (Kriegel et al., 2013) due to increased winter snowmelt and more precipitation falling as rain in addition to increases in precipitation in some basins (Table SM2.9). There is robust evidence (high agreement) that summer runoff will decline over the 21st century in many basins for all emission scenarios, for example, in Western Canada and USA (Shrestha et al., 2017), the European Alps (Jenicek et al., 2018), High Mountain Asia (Prasch et al., 2013; Engelhardt et al., 2017) and the tropical Andes (Baraer et al., 2012), due to less snowfall and decreases in glacier melt after peak water. A global-scale projection suggests that decline in glacier runoff by 2100 (RCP8.5) may reduce basin runoff by $10 \%$ or more in at least one month of the melt season in several large river basins, especially in High Mountain Asia during dry seasons, despite glacier cover of less than a few percent (Huss and Hock, 2018).

Projected changes in annual runoff in glacier dominated basins are complex including increases and decreases over the 21st century for all scenarios depending on the time period and the timing of peak water (high confidence) (Figure 2.6). Local and regional-scale projections in High Mountain Asia, the European Alps, and Western Canada and USA suggest that peak water will generally be reached before or around the middle of the century. These finding are consistent with results from global-scale modelling of glacier runoff
(Bliss et al., 2014; Huss and Hock, 2018) indicating generally earlier peak water in regions with little ice cover and smaller glaciers (e.g., Low Latitudes, European Alps and Pyrenees, and the Caucasus) and later peak water in regions with extensive ice cover and large glaciers (e.g., Alaska, Southern Andes). In some regions (e.g., Iceland) peak water from most glacier area is projected to occur earlier for RCP2.6 than RCP8.5, caused by decreasing glacier runoff as glaciers find a new equilibrium. In contrast melt-driven glacier runoff continues to rise for the higher emission scenario. There is very high confidence that spring peak runoff in many snow-dominated basins around the world will occur earlier in the year, up to several weeks, by the end of the century caused by earlier snowmelt (e.g., Coppola et al., 2014; Bard et al., 2015; Yucel et al., 2015; Islam et al., 2017; Sultana and Choi, 2018).

In addition to changes in ice and snow melt, changes in other variables such as precipitation and evapotranspiration due to atmospheric warming or vegetation change affect runoff amounts and timing (e.g., Bocchiola, 2014; Lutz et al., 2016). Changes in melt water from ice and snow often dominates the runoff response to climate change at higher elevations, while changes in precipitation and evapotranspiration become increasingly important at lower elevations (Kormann et al., 2015). Permafrost thaw may affect runoff by releasing water from ground ice (Jones et al., 2018) and indirectly by changing hydrological pathways or ground water recharge as permafrost degrades (Lamontagne-Hallé et al., 2018). The relative importance of runoff from thawing permafrost compared to runoff from melting glaciers is expected to be greatest in arid areas where permafrost tends to be more abundant (Gruber et al., 2017). Because glaciers react more rapidly to climate change than permafrost, runoff in some mountain landscapes may become increasingly affected by permafrost thaw in the future (Jones et al., 2018).

In summary, there is very high confidence that glacier and snow cover decline have affected and will continue to change the amounts and seasonality of river runoff in many snow-dominated and/or glacier-fed river basins. The average winter runoff is expected to increase (high confidence), and spring peak maxima will occur earlier (very high confidence). Although observed and projected trends in annual runoff vary substantially among regions and can even be opposite in sign, there is high confidence that average annual runoff from glaciers will have reached a peak, with declining runoff thereafter, at the latest by the end of the 21st century in all regions regardless emission scenario. The projected changes in runoff are expected to affect downstream water management, related hazards and ecosystems (Section 2.3.2, 2.3.3). 
RCP2.6

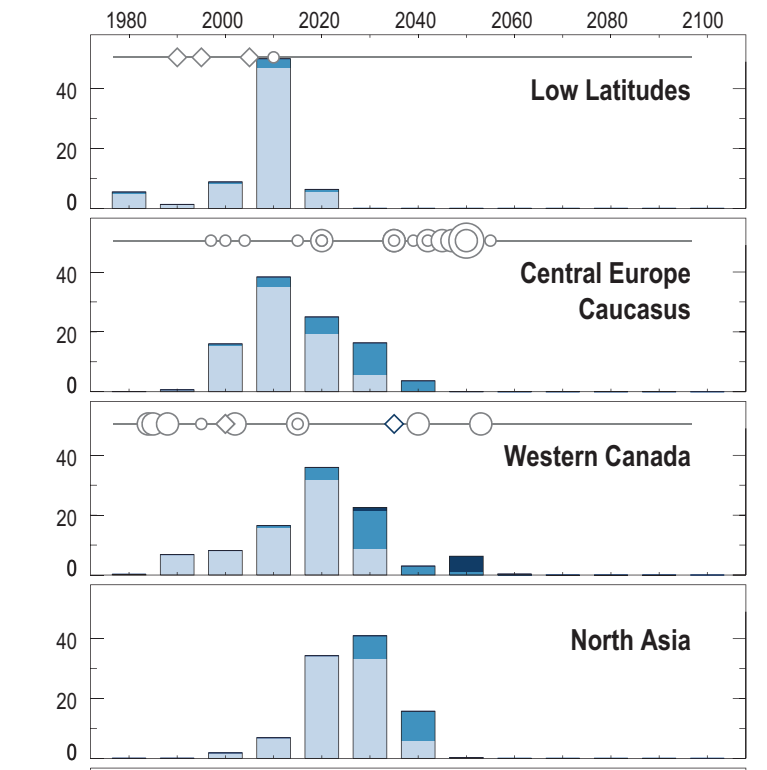

ত্য

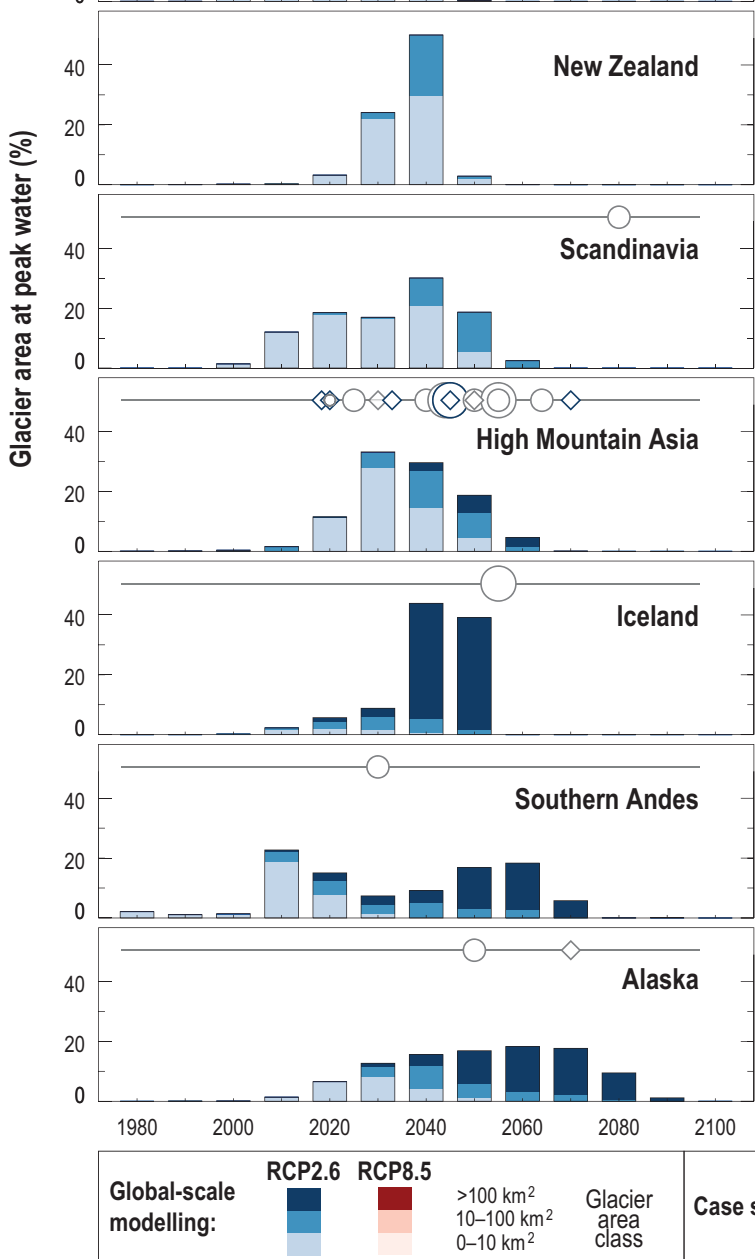

RCP8.5
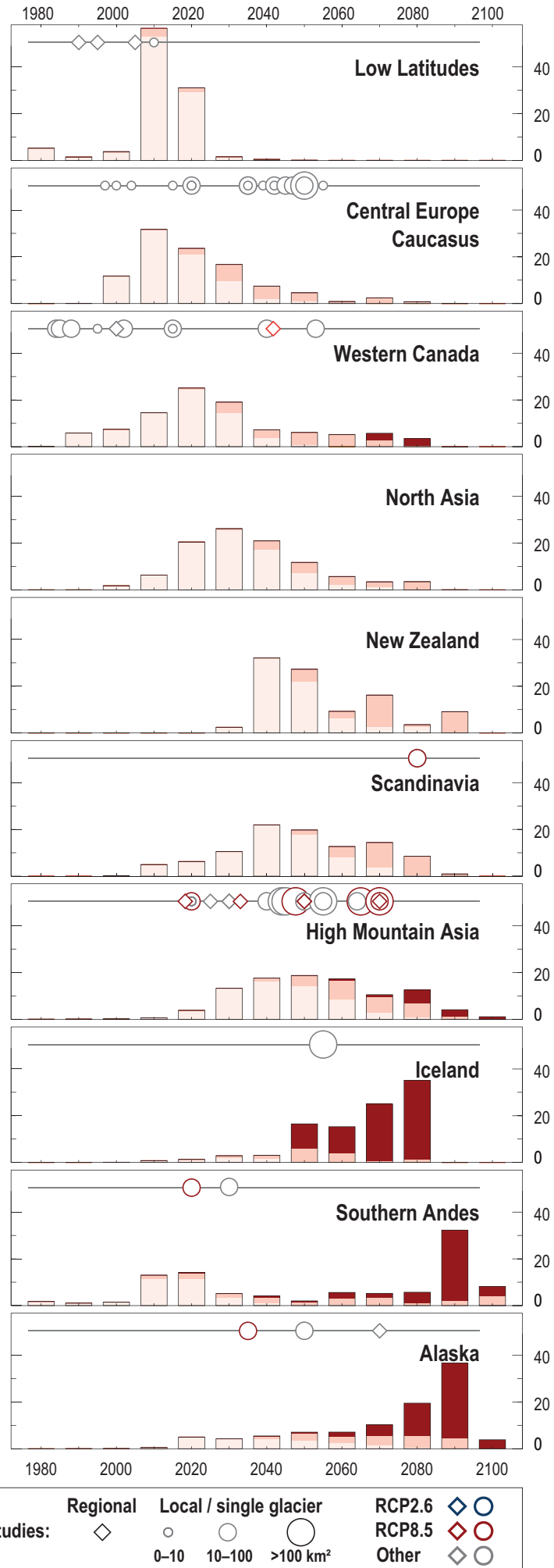

Figure 2.6 Timing of peak water from glaciers in different regions (Figure 2.1) under two emission scenarios for Representative Concentration Pathways RCP2.6 and RCP8.5. Peak water refers to the year when annual runoff from the initially glacier-covered area will start to decrease due to glacier shrinkage after a period of melt induced increase. The bars are based on Huss and Hock (2018) who used a global glacier model to compute the runoff of all individual glaciers in a region until year 2100 based on 14 General Circulation Models (GCMs). Depicted is the area of all glaciers that fall into the same 10-year peak water interval expressed as a percentage of each region's total glacier area, i.e., all bars for the same RCP sum up to $100 \%$ glacier area. Shadings of the bars distinguish different glacier sizes indicating a tendency for peak water to occur later for larger glaciers. Circles/diamonds mark timing of peak water from individual case studies based on observations or modelling (Table SM2.10). Circles refer to results from individual glaciers regardless of size or a collection of glaciers covering $<150 \mathrm{~km}^{2}$ in total, while diamonds refer to regional-scale results from a collection of glaciers with $>150 \mathrm{~km}^{2}$ glacier coverage. Case studies based on observations or scenarios other than RCP2.6 and RCP8.5 are shown in both the left and right set of panels. 
Frequently Asked Questions

\section{FAQ 2.1 How does glacier shrinkage affect river runoff further downhill?}

Glaciers supply water that supports human communities both close to the glacier and far away from the glacier, for example for agriculture or drinking water. Rising temperatures cause mountain glaciers to melt and change the water availability. At first, as the glacier melts, more water runs downhill away from the glacier. However, as the glacier shrinks, the water supply will diminish and farms, villages and cities might lose a valuable water source.

Melting glaciers can affect river runoff, and thus freshwater resources available to human communities, not only close to the glacier but also far from mountain areas. As glaciers shrink in response to a warmer climate, water is released from long-term glacial storage. At first, glacier runoff increases because the glacier melts faster and more water flows downhill from the glacier. However, there will be a turning point after several years or decades, often called 'peak water', after which glacier runoff and hence its contribution to river flow downstream will decline (FAQ 2.1; Figure 1). Peak water runoff from glaciers can exceed the amount of initial yearly runoff by $50 \%$ or more. This excess water can be used in different ways, such as for hydropower or irrigation. After the turning point, this additional water decreases steadily as the glacier continues to shrink, and eventually stops when the glacier has disappeared, or retreated to higher elevations where it is still cold enough for the glacier to survive. As a result, communities downstream lose this valuable additional source of water. Total amounts of river runoff will then depend mainly on rainfall, snowmelt, ground water and evaporation.

Furthermore, glacier decline can change the timing in the year and day when the most water is available in rivers that collect water from glaciers. In mid- or high latitudes, glacier runoff is greatest in the summer, when the glacier ice continues to melt after the winter snow has disappeared, and greatest during the day when air temperature and solar radiation are at their highest (FAQ 2.1, Figure 1). As peak water occurs, more intense glacier melt rates also increase these daily runoff maxima significantly. In tropical areas, such as parts of the Andes, seasonal air temperature variations are small, and alternating wet and dry seasons are the main control on the amount and timing of glacier runoff throughout the year.

The effects of glaciers on river runoff further downhill depend on the distance from the glacier. Close to the glaciers (e.g., within several kilometres), initial increases in yearly glacier runoff until peak water followed by decreases can affect water supply considerably, and larger peaks in daily runoff from the glaciers can cause floods. Further away from the glaciers the impact of glacier shrinkage on total river runoff tends to become small or negligible. However, the melt water from glaciers in the mountains can be an important source of water in hot and dry years or seasons when river runoff would otherwise be low, and thereby also reducing variability in total river runoff from year to year, even hundreds of kilometres away from the glaciers. Other components of the water cycle such as rainfall, evaporation, groundwater and snowmelt can compensate or strengthen the effects of changes in glacier runoff as the climate changes. 

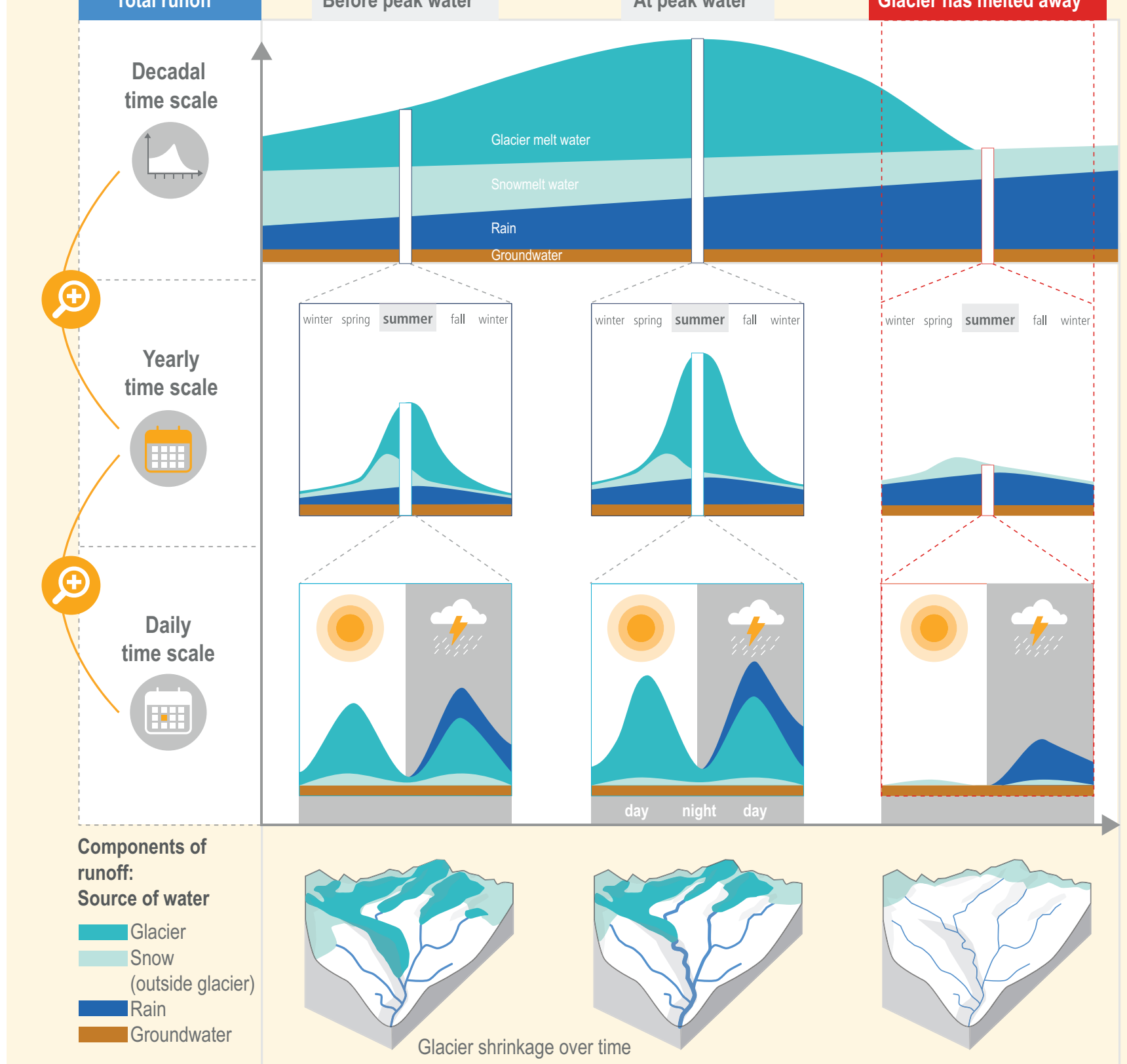

FAQ 2.1, Figure 1 | A simplified overview of changes in runoff from a river basin with large (e.g., $>50 \%$ ) glacier cover as the glaciers shrink, showing the relative amounts of water from different sources - glaciers, snow (outside the glacier), rain and groundwater. Three different time scales are shown: annual runoff from the entire basin (upper panel); runoff variations over one year (middle panel) and variations during a sunny then a rainy summer day (lower panel). Note that seasonal and daily runoff variations are different before, during and after peak flow. The glacier's initial negative annual mass budget becomes more negative over time until eventually the glacier has melted away. This is a simplified figure so permafrost is not addressed specifically and the exact partitioning between the different sources of water will vary between river basins.

\subsubsection{Water Quality}

Glacier decline can influence water quality by accelerating the release of stored anthropogenic legacy pollutants, with impacts to downstream ecosystem services. These legacy pollutants notably include persistent organic pollutants (POPs), particularly polychlorinated biphenyls (PCBs) and dichlorodiphenyl-trichloroethane (DDT), polycyclic aromatic hydrocarbons, and heavy metals (Hodson, 2014) and are associated with the deposition and release of black carbon. There is limited evidence that some of these pollutants found in surface waters in the Gangetic Plain during the dry season originate from Himalayan glaciers (Sharma et al., 2015), and glaciers in the European Alps store the largest known quantity of POPs in the Northern Hemisphere (Milner et al., 2017). Although their use has declined or ceased 
worldwide, PCBs have been detected in runoff from glacier melt due to the lag time of release from glaciers (Li et al., 2017). Glaciers also represent the most unstable stores of DDT in European and other mountain areas flanking large urban centres and glacier derived DDT is still accumulating in lake sediments downstream from glaciers (Bogdal et al., 2010). However, bioflocculation (the aggregation of dispersed organic particles by the action of organisms) can increase the residence time of these contaminants stored in glaciers thereby reducing their overall toxicity to freshwater ecosystems (Langford et al., 2010). Overall the effect on freshwater ecosystems of these contaminants is estimated to be low (medium confidence) (Milner et al., 2017).

Of the heavy metals, mercury is of particular concern and an estimated 2.5 tonnes has been released by glaciers to downstream ecosystems across the Tibetan Plateau over the last 40 years (Zhang et al., 2012). Mercury in glacial silt, originating from grinding of rocks as the glacier flows over them, can be as large or larger than the mercury flux from melting ice due to anthropogenic sources deposited on the glacier (Zdanowicz et al., 2013). Both glacier erosion and atmospheric deposition contributed to the high rates of total mercury export found in a glacierised watershed in coastal Alaska (Vermilyea et al., 2017) and mercury output is predicted to increase in glacierised mountain catchments (Sun et al., 2017; Sun et al., 2018b) (medium confidence). However, a key issue is how much of this glacier-derived mercury, largely in the particulate form, is converted to toxic methyl mercury downstream. Methyl mercury can be incorporated into aquatic food webs in glacier streams (Nagorski et al., 2014) and bio-magnify up the food chain (Lavoie et al., 2013). Water originating from rock glaciers can also contribute other heavy metals that exceed guideline values for drinking water quality (Thies et al., 2013). In addition, permafrost degradation can enhance the release of other trace elements (e.g., aluminium, manganese and nickel) (Colombo et al., 2018). Indeed, projections indicate that all scenarios of future climate change will enhance the mobilisation of metals in metamorphic mountain catchments (Zaharescu et al., 2016). The release of toxic contaminants, particularly where glacial melt waters are used for irrigation and drinking water in the Himalayas and the Andes, is potentially harmful to human health both now and in the future (Hodson, 2014) (medium confidence).

Soluble reactive phosphorus concentrations in rivers downstream of glaciers are predicted to decrease with declining glacier coverage (Hood et al., 2009) as a large percentage is associated with glacier-derived suspended sediment (Hawkings et al., 2016). In contrast, dissolved organic carbon (DOC), dissolved inorganic nitrogen and dissolved organic nitrogen concentrations in pro-glacial rivers is projected to increase this century due to glacier shrinkage (Hood et al., 2015; Milner et al., 2017) (robust evidence, medium agreement). Globally, mountain glaciers are estimated to release about $0.8 \mathrm{Tera}_{\mathrm{g} \mathrm{C} \mathrm{yr}^{-1}}$ (Li et al., 2018) of highly bioavailable DOC that may be incorporated into downstream food webs (Fellman et al., 2015; Hood et al., 2015). Loss rates of DOC from glaciers in the high mountains of the Tibetan Plateau were estimated to be $\sim 0.19$ Tera $\mathrm{g} \mathrm{C} \mathrm{yr}^{-1}$, (Li et al., 2018) higher than other regions suggesting that DOC is released more efficiently from Asian mountain glaciers (Liu et al., 2016). Glacier DOC losses are expected to accelerate as they shrink, leading to a cumulative annual loss of roughly 15 Tera $\mathrm{C} \mathrm{yr}^{-1}$ of glacial DOC by 2050 from melting glaciers and ice sheets (Hood et al., 2015). Permafrost degradation is also a major and increasing source of bioavailable DOC (Abbott et al., 2014; Aiken et al., 2014). Major ions calcium, magnesium, sulphate and nitrate (Colombo et al., 2018) are also released by permafrost degradation as well as acid drainage leaching into alpine lakes (llyashuk et al., 2018).

Increasing water temperature has been reported in some high mountain streams (e.g., Groll et al., 2015; Isaak et al., 2016) due to decreases in glacial runoff, producing changes in water quality and species richness (Section 2.3.3). In contrast, water temperature in regions with extensive glacier cover are expected to show a transient decline, due to an enhanced cooling effect from increased glacial melt water (Fellman et al., 2014).

In summary, changes in the mountain cryosphere will cause significant shifts in downstream nutrients (DOC, nitrogen, phosphorus) and influence water quality through increases in heavy metals, particularly mercury, and other legacy contaminants (medium evidence, high agreement) posing a potential threat to human health. These threats are more focused where glaciers are subject to substantial pollutant loads such as High Mountain Asia and Europe, rather than areas like Alaska and Canada.

\subsubsection{Key Impacts and Vulnerability}

\subsection{Hydropower}

Hydropower comprises about $16 \%$ of electricity generation globally but close to $100 \%$, in many mountainous countries (Hamududu and Killingtveit, 2012; IHA, 2018). It represents a significant source of revenue for mountainous regions (Gaudard et al., 2016). Due to the dependence on water resources as key input, hydropower operations are expected to be affected by changes in runoff from glaciers and snow cover (Section 2.3.1.1, FAQ 2.1). Both increases and decreases in annual and/or seasonal water input to hydropower facilities have been recorded in several high mountain regions, for example, in Switzerland (Hänggi and Weingartner, 2012; Schaefli et al., 2019), Canada (Jost et al., 2012; Jost and Weber, 2013), Iceland (Einarsson and Jónsson, 2010) and High Mountain Asia (Ali et al., 2018). However, there is only limited evidence (medium agreement) that changes in runoff have led to changes in hydropower plant operation. For example, in Iceland, the National Power Company observed in 2005 that flows into their energy system were greater than historical flows. By incorporating the most recent runoff data into strategies for reservoir management it was possible to increase production capacity (Braun and Fournier, 2016).

There is robust evidence (medium agreement) that water input to hydropower facilities will change in the future due to cryosphere-related impacts on runoff (Section 2.3.1.1). For example, in the Skagit river basin in British Columbia and Northern Washington (Lee et al., 2016) and in California (Madani and Lund, 2010) projections (SRESA1B) show more runoff in winter and less in summer. In India, snow and glacier runoff to hydropower plants is projected to decline in several basins (Ali et al., 2018). In some cases, catchments that are close together 
are projected to evolve in contrasting directions in terms of runoff, for example in the European Alps (Gaudard et al., 2013; Gaudard et al., 2014). Increased runoff due to changes in the cryosphere will increase the risk of overflows (non-productive discharge), particularly during winter and spring melt, with the greatest impacts on run-ofriver power plants (e.g., in Canada; Minville et al., 2010; Warren and Lemmen, 2014) (medium confidence).

There is medium evidence (high agreement) that changes in glacierand moraine-dammed lakes, and changes in sediment supply will affect hydropower generation (Colonia et al., 2017; Hauer et al., 2018). Many glacier lakes have increased in volume, and can damage hydropower infrastructure when they empty suddenly (Engeset et al., 2005; Jackson and Ragulina, 2014; Carrivick and Tweed, 2016) (Section 2.3.2; Figure 2.7). If large enough, hydropower reservoirs can reduce the downstream negative impacts of changes in the cryosphere by storing and providing freshwater during hot, dry periods or by alleviating the effects of glacier floods (Jackson and Ragulina, 2014; Colonia et al., 2017). In mountain rivers, sediment volume and type depend on connectivity between hillslopes and the valley floor (Carrivick et al., 2013), glacier activity (Lane et al., 2017) and on water runoff regime feedbacks with river channel dynamics (Schmidt and Morche, 2006). An increase in suspended sediment loading under current reservoir operating policies is projected for some hydropower facilities, for example, in British Columbia and Northern Washington (Lee et al., 2016).

Only a few studies have addressed the economic effects on hydropower due directly to changes in the cryosphere. For example in Peru, Vergara et al. (2007) studied the effect of both reduced glacier runoff and runoff with no glacier input once the glaciers have completely melted for the Cañón del Pato hydropower plant in Peru, and found an economic cost of between 5-20 million USD $\mathrm{yr}^{-1}$, with the lower figure for the cost of energy paid to the producer and the higher figure the society cost. Costs calculated for all of Peru, where $\sim 80 \%$ of electricity comes from hydropower range from 60-212 million USD $\mathrm{yr}^{-1}$. If the cost of rationing energy is considered, the national cost is estimated as 1,500 million USD $\mathrm{yr}^{-1}$.

Other factors than changes in the cryosphere, such as market policies and regulation, may have greater significance for socioeconomic development of hydropower in the future (Section 2.3.1.4, Gaudard et al., 2016). Hence, despite the efforts of hydropower agencies and regulatory bodies to quantify changes or to develop possible adaptation strategies (IHA, 2018), only a few organisations are incorporating current knowledge of climate change into their investment planning. The World Bank uses a decision tree approach to identify potential vulnerabilities in a hydropower project incurred from key uncertain factors and their combinations (Bonzanigo et al., 2015).

\subsection{Agriculture}

High mountains have supported agricultural livelihoods for centuries. Rural communities are dependent on adequate levels of soil moisture at planting time, derived in part in many cases from irrigation water which includes glacier and snowmelt water; as a result, they are exposed to risk which stems from cryosphere changes (high confidence) (Figure 2.8). The relative poverty of many mountain communities contributes to their vulnerability to the impacts of these cryosphere changes (McDowell et al., 2014; Carey et al., 2017; Rasul and Molden, 2019) (medium evidence, high agreement). Glacier and snowmelt water contribute irrigation water to adjacent lowlands as well. Pastoralism, an important livelihood strategy in mountain regions, is also impacted by cryosphere changes, but described in Section 2.3.7.

There is medium evidence (medium agreement) that reduction in streamflow due to glacier retreat or reduced snow cover has led to reduced water availability for irrigation of crops and declining agricultural yields in several mountain areas (Table SM2.11), for example in the tropical Andes (e.g., Bury et al., 2011) and High Mountain Asia (e.g., Nüsser and Schmidt, 2017). In the Southern Andes, increased streamflow in the Elqui River in Chile, due to glacier retreat or changing snow cover, has led to increased water availability for irrigation and increased agricultural yields (Young et al., 2010).

In addition to the effects on agriculture of changing availability of irrigation water, reductions in snow cover can also impact agriculture through its direct effects on soil moisture, as reported for Nepal, where lesser snow cover has led to the drying of soils and lower yields of potatoes and fodder (Smadja et al., 2015). Agriculture in high mountain areas is sensitive to other climatic drivers as well. Rising air temperatures increase crop evapotranspiration, thus increasing water demand for crop production to maintain optimal yield (Beniston and Stoffel, 2014). They are also associated with upslope movement of cropping zones, which favours some farmers in high mountain areas, who are increasingly able to cultivate new crops, such as onions, garlic and apples in Nepal (Huntington et al., 2017; Hussain et al., 2018), and maize in Ecuador (Skarbø and VanderMolen, 2014). Dry spells and unseasonal frosts have also impacted agriculture in Peru (Bury et al., 2011).

Adaptation activities in mountain agriculture related at least partially to cryospheric changes are detailed in Table SM2.12 and their geographic spread shown in Figure 2.9. Agriculture in these areas is sensitive to non-climate drivers as well, such as market forces and political pressures (Montana et al., 2016; Sietz and Feola, 2016; Figueroa-Armijos and Valdivia, 2017) and shifts in water governance (Rasmussen, 2016). The majority of the adaptation activities are autonomous, though some are planned or carried out with support from national governments, non-governmental organisations (NGOs), or international aid organisations. Though many studies report on benefits from these activities which accrue to community members as increased harvests and income, systematic evaluations of these adaptation strategies are generally lacking. A range of factors, discussed below, place barriers which limit the scale and scope of these activities in the mountain agricultural sector, including a lack of finance and technical knowledge, low adaptive capacity within communities, ill-equipped state organisations, ambiguous property rights and inadequate institutional and market support (medium evidence, high agreement). Section 2.3.7 examines two other responses to decreasing irrigation water: wage labour migration, which often serves as an adaptation strategy, and displacement of 
entire communities, an indication of the limits to adaptation - this displacement is also due in some cases to natural hazards.

To cope with the reduced water supplies, planted areas have been reduced in a number of different places in Nepal (Gentle and Maraseni, 2012; Sujakhu et al., 2016). Adaptation responses within irrigation systems include the adoption of new irrigation technologies or upgrading existing technologies, adopting water conservation measures, water rationing, constructing water storage infrastructure, and change in cropping patterns (Rasul et al., 2019; Figure 2.9). Water delivery technologies which reduce loss are adopted in Chile (Young et al., 2010) and Peru (Orlove et al., 2019). Similarly, greenhouses have been adopted in Nepal (Konchar et al., 2015) to reduce evapotranspiration and frost damage, though limited access to finance is a barrier to these activities. Box 2.3 describes innovative irrigation practices in India. Local pastoral communities have responded to these challenges with techniques broadly similar to those in agricultural settings by expanding irrigation facilities, for example, in Switzerland (Fuhrer et al., 2014). In addition to adopting new technologies, some water users make investments to tap more distant sources of irrigation water. Cross-Chapter Box 3 in Chapter 1 discusses such efforts in Northern Pakistan, where landslides, associated with cryosphere change, have also damaged irrigation systems.

The adoption of new crops and varieties is an adaptation response found in several regions. Farmers in northwest India have increased production of lentils and vegetables, which provide important nutrients to the local diet, with support from government watershed improvement programs which help address decreased availability of irrigation water, though stringent requirements for participation in the programs have limited access by poor households to this assistance (Dame and Nüsser, 2011). Farmers who rely on irrigation in the Naryn River basin in Kyrgyzstan have shifted from the water intensive fruits and vegetables to fodder crops such as barley and alfalfa, which are more profitable. Upstream communities, with greater access to water and more active local institutions, are more willing to experiment with new crops than those further downstream (Hill et al., 2017). In other areas, crop choices also reflect responses to rising temperatures along with new market opportunities such as the demand for fresh vegetables by tourists in Nepal (Konchar et al., 2015; Dangi et al., 2018) and the demand for roses in urban areas in Peru (SENASA, 2017). Indigenous knowledge and local knowledge (Cross-Chapter Box 4 in Chapter 1), access to local and regional seed supply networks, proximity to agricultural extension and support services also facilitate the adoption of new crops (Skarbø and VanderMolen, 2014).
Local institutions and embedded social relations play a vital role in enabling mountain communities to respond to the impacts of climate driven cryosphere change. Indigenous pastoral communities who have tapped into new water sources to irrigate new areas have also strengthened the control of access to existing irrigated pastures in Peru (Postigo, 2014) and Bolivia (Yager, 2015). In an example of indigenous populations in the USA, two tribes who share a large reservation in the Northern Rockies rely on rivers which receive glacier melt water to irrigate pasture, and maintain fisheries, domestic water supplies, and traditional ceremonial practices. Tribal water managers have sought to install infrastructure to promote more efficient water use and protect fisheries, but these efforts have been impeded by land and water governance institutions in the region and by a history of social marginalisation (McNeeley, 2017).

High mountain communities have sought new financial resources from wage labour (Section 2.3.7), tourism (Mukherji et al., 2019) and government sources to support adaptation activities. Local water user associations in Kyrgyzstan and Tajikistan have adopted less water intensive crops and reorganised the use and maintenance of irrigation systems, investing government relief payments after floods (Stucker et al., 2012). Similar measures are reported from India and Pakistan (Dame and Mankelow, 2010; Clouse, 2016; Nüsser and Schmidt, 2017), Nepal (McDowell et al., 2013) and Peru (Postigo, 2014). In contrast, fewer adaptation measures have been adopted in Uzbekistan, due to low levels of capital availability and to agricultural policies, including centralised water management, crop production quotas and weak agricultural extension, which limit the response capacity of farmers (Aleksandrova et al., 2014).

Lowland agricultural areas which receive irrigation water from rivers fed by glacier melt and snowmelt are projected to face negative impacts in some regions (limited evidence, high agreement). In the Rhone basin in Switzerland, many irrigated pasture areas are projected to face water deficits by 2050, under the A1B scenario (Fuhrer et al., 2014; Cross Chapter Box 1 in Chapter 1). For California and the southwestern USA, a shift to peak snowmelt earlier in the year would create more frequent floods, and a reduced ability of existing reservoirs to store water by 2050 under RCP8.5 (Pagán et al., 2016) and by 2100 under RCP2.6, RCP4.5 and RCP8.5 (Pathak et al., 2018). The economic values of these losses have been estimated at 10.8-48.6 billion USD by around 2050 (Sturm et al., 2017). A similar transition to runoff peaks earlier in the year by 2100 under RCP2.6, RCP4.5 and RCP8.5, creating challenges for management of irrigation water, has been reported for the countries in central Asia which are dependent on snow cover and glaciers of the Tien Shan (Xenarios et al., 2018). In India and Pakistan, where over 100 million farmers receive irrigation from the Indus and Ganges Rivers, which also have significant inputs from glaciers and snowmelt, also face risks of decreasing water supplies from cryosphere change by 2100 (Biemans et al., 2019; Rasul and Molden, 2019). 


\section{Box 2.3 | Local Responses to Water Shortage in northwest India}

Agriculture in Ladakh, a cold arid mountain region $\left(\sim 100,000 \mathrm{~km}^{2}\right)$ in the western Himalaya of India with median elevation of 3,350 $\mathrm{m}$ a.s.l. and mean annual precipitation of less than $100 \mathrm{~mm}$, is highly dependent on streams for irrigation in the agricultural season in the spring and summer (Nüsser et al., 2012; Barrett and Bosak, 2018). Glaciers in Ladakh, largely located at 5,000-6,000 m a.s.l. and small in size have retreated at least since the late 1960s although less pronounced than in many other Himalayan regions (Chudley et al., 2017; Schmidt and Nüsser, 2017). However, the effect of glaciers on streamflow in Ladakh is poorly constrained, and measurements on changes in runoff and snow cover are lacking (Nüsser et al., 2018).

To cope with seasonal water scarcity at critical times for irrigation, villagers in the region have developed four types of artificial ice reservoirs: basins, cascades, diversions and a form known locally as ice stupas. All these types of ice reservoirs capture water in the autumn and winter, allowing it to freeze, and hold it until spring, when it melts and flows down to fields (Clouse et al., 2017; Nüsser et al., 2018). In this way, they retain a previously unused portion of the annual flow and facilitate its use to supplement the decreased flow in the following spring (Vince, 2009; Shaheen, 2016). Frozen basins are formed from water which is conveyed across a slope through channels and check dams to shaded surface depressions near the villages. Cascades and diversions direct water to pass over stone walls, slowing its movement and allowing it to freeze. Ice stupas direct water through pipes into fountains, where it freezes into conical shapes (Box 2.3 Figure 1). These techniques use local materials and draw on local knowledge (Nüsser and Baghel, 2016).

A study examined 14 ice reservoirs, including ice stupas, and concluded that they serve as 'site-specific water conservation strategies; and that they can be regarded as appropriate local technologies to reduce seasonal water scarcity at critical times (Nüsser et al., 2018). It listed the benefits of ice reservoirs as improved water availability in spring, reduction of seasonal water scarcity and resulting crop failure risks, and the possibility of growing cash crops. However, the study questioned their usefulness as a long-term adaptation strategy, because their operation depends on winter runoff and freeze-thaw cycles, both of which are sensitive to interannual variability, and often deviate from the optimum range required for effective functioning of the reservoirs. It also raised questions about the financial costs and labour requirements, which vary across the four types of ice reservoirs.

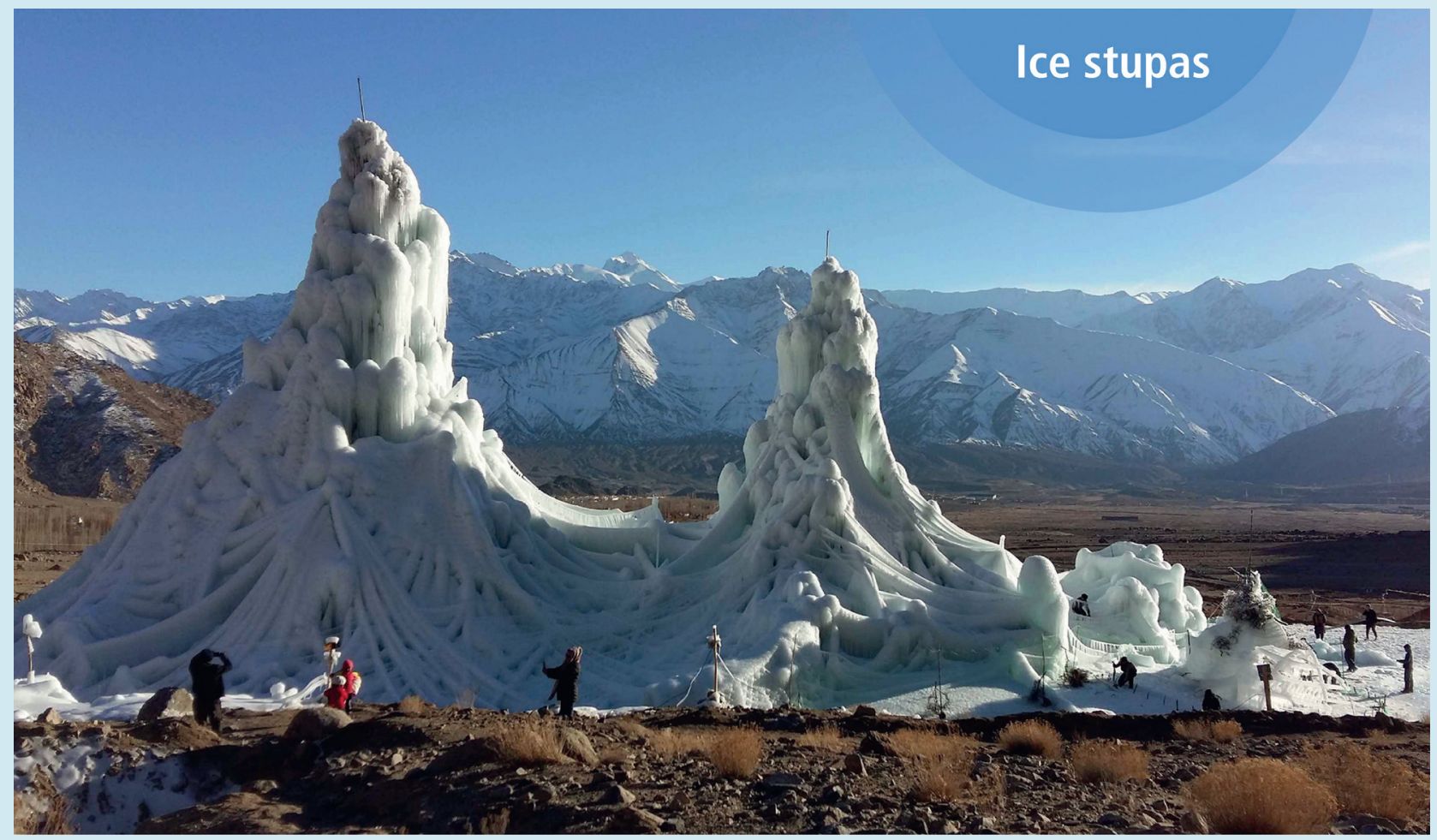

Box 2.3, Figure 1 | Ice stupas in Ladakh, India (Photo: Padma Rigzin) 


\subsection{Drinking water supply}

Only a few studies provide detailed empirical assessments of the effects of cryosphere change on the amounts of drinking water supply. Decreases in drinking water supplies due to reduced glacier and snowmelt water have been reported for rural areas in the Nepal Himalaya (McDowell et al., 2013; Dangi et al., 2018), but the tropical Andes have received the most attention, including both urban conglomerates and some rural areas, where water resources are especially vulnerable to climate change due to water scarcity and increased demands (Chevallier et al., 2011; Somers et al., 2018), amidst rapidly retreating glaciers (Burns and Nolin, 2014).

The contribution of glacier water to the water supply of La Paz, Bolivia, between 1963-2006 was assessed at 15\% annually and 27\% during the dry season (Soruco et al., 2015), though rising as high as $86 \%$ during extreme drought months (Buytaert and De Bièvre, 2012). Despite a $50 \%$ area loss, the glacier retreat has not contributed to reduced water supplies for the city, because increased melt rates have compensated for reductions in glacier volume. However, for a complete disappearance of the glaciers, assuming no change in precipitation, a reduction in annual runoff by $12 \%$ and $24 \%$ in the dry season was projected (Soruco et al., 2015) similar to reductions projected by 2050 under a RCP8.5 scenario for a basin in southern Peru (Drenkhan et al., 2019). Huaraz and Huancayo in Peru are other cities with high average contribution of melt water to surface water resources (up to 20\%; Buytaert et al., 2017) and rapid glacier retreat in their headwaters (Rabatel et al., 2013).

Overall, risks to water security and related vulnerabilities are highly heterogeneous varying even at small spatial scales with populations closer to the glaciers being more vulnerable, especially during dry months and droughts (Buytaert et al., 2017; Mark et al., 2017). A regional-scale modelling study including all of Bolivia, Ecuador and Peru (Buytaert et al., 2017) estimated that roughly 390,000 domestic water users, mostly in Peru, rely on a high ( $>25 \%$ ) long-term average contribution from glacier melt, with this number rising to almost 4 million in the driest month of a drought year. Despite high confidence in declining longer-term melt water contributions from glaciers in the tropical Andes (Figure CB6.1), major uncertainties remain how these will affect future human water use. Regional-scale water balance simulations forced by multi-model climate projections (Buytaert and De Bièvre, 2012), suggest a relatively limited effect of glacier retreat on water supply in four major cities (Bogota, La Paz, Lima, Quito) due to the dominance of human factors influencing water supply (Carey et al., 2014; Mark et al., 2017; Vuille et al., 2018), though uncertainties are large. Population growth and limited funding for infrastructure maintenance exacerbate water scarcity, though water managers have established programs in Quito and in Huancayo and the Santa and Vilcanota basins (Peru) to improve water management through innovations in grey infrastructure and ecosystem-based adaptations (Buytaert and De Bièvre, 2012; Buytaert et al., 2017; Somers et al., 2018).

In summary, there is limited evidence (medium agreement) that glacier decline places increased risks to drinking water supply. In the Andes, future increases in water demand due to population growth and other socioeconomic stressors are expected to outpace the impact of climate change induced changes on water availability regardless the emission scenario.

\subsubsection{Water Governance and Response Measures}

Cryospheric changes induced by climate change, and their effects on hydrological regime and water availability, bear relevance for the management and governance of water as a resource for communities and ecosystems (Hill, 2013; Beniston and Stoffel, 2014; Carey et al., 2017), particularly in areas where snow and ice contribute significantly to river runoff (medium confidence) (Section 2.3.1.1). However, water availability is one aspect relevant for water management and governance, given that multiple and diverse decision making contexts and governance approaches and strategies can influence how the water resource is accessed and distributed (medium confidence) (De Stefano et al., 2010; Beniston and Stoffel, 2014).

A key risk factor that influences how water is managed and governed, rests on existing and unresolved conflicts that may or may not necessarily arise exclusively from demands over shared water resources, raising tensions within and across borders in river basins influenced by snow and glacier melt (Valdés-Pineda et al., 2014; Bocchiola et al., 2017). For example, in Central Asia, competing demand for water for hydropower and irrigation between upstream and downstream countries has raised tensions (Bernauer and Siegfried, 2012; Bocchiola et al., 2017). Similarly, competing demand for water is also reported in Chile (Valdés-Pineda et al., 2014) and in Peru (Vuille, 2013; Drenkhan et al., 2015). Since AR5, some studies have examined the impacts and risks related to projections of cryosphere-related changes in streamflow in transboundary basins in the 21st century, and suggest that these changes create barriers in effectively managing water in some settings (medium confidence). For instance, within the transnational Indus River basin, climate change impacts may reduce streamflow by the end of this century, thus putting pressure on established water sharing arrangements between nations (Jamir, 2016) and subnational administrative units (Yang et al., 2014b). In this basin, management efforts may be hampered by current legal and regulatory frameworks for evaluating new dams, which do not take into account changes in streamflow that may result from climate change (Raman, 2018). Within the transnational Syr Darya and Amu Darya basins in Central Asia, competition for water between multiple uses, exacerbated by reductions in flow later in this century, may hamper future coordination (Reyer et al., 2017; Yu et al., 2019). However, other evidence from Central Asia suggests that relative water scarcity may not be the only factor to exacerbate conflict in this region (Hummel, 2017). Overall, there is medium confidence in the ability to meet future water demands in some mountain regions, given the combined uncertainties associated with accurate projections of water supply in terms of availability and the diverse sociocultural and political contexts in which decisions on water access and distribution are taken.

Since AR5, several studies highlight that integrated water management approaches, focused on the multipurpose use of water that includes water released from the cryosphere, are important as adaptation measures, particularly for sectors reliant on this water 
source to sustain energy production, agriculture, ecosystems and drinking water supply (Figure 2.9). These measures, backed by effective governance arrangements to support them, demonstrate an ability to address increasing challenges to water availability arising from climate change in the mountain cryosphere, providing co-benefits through the optimisation of storage and the release of water from high mountain reservoirs (medium confidence). Studies in Switzerland (e.g., Haeberli et al., 2016; Brunner et al., 2019), Peru (e.g., Barriga Delgado et al., 2018; Drenkhan et al., 2019), Central Asia (Jalilov et al., 2018) and Himalaya (Molden et al., 2014; Biemans et al., 2019) highlight the potential of water reservoirs in high mountains, including new reservoirs located in former glacier beds, alleviating seasonal water scarcity for multiple water usages. However, concerns are also raised in the environmental literature about their actual and potential negative impacts on local ecosystems and biodiversity hotspots, such as wetlands and peat bogs, which have been reported for small high mountain reservoirs, for example, in the European Alps (Evette et al., 2011) and for large dam construction projects in High Mountain Asia (e.g., Dharmadhikary, 2008).

Transboundary cooperation at regional scales are reported to further support efforts that address the potential risks to water resources in terms of its availability and its access and distribution governance (Dinar et al., 2016). Furthermore, the UN 2030 Agenda and its Sustainable Development Goals (SDGs) (UN, 2015) may offer additional prospects to strengthen water governance under a changing cryosphere, given that monitoring and reporting on key water-related targets and indicators, and their interaction across other SDGs, direct attention to the provision of water as a key condition for development (Section 2.4). However, there is limited evidence to date to assess their effectiveness on an evidentiary basis.

\subsubsection{Landslide, Avalanche and Flood Hazards}

High mountains are particularly prone to hazards related to snow, ice and permafrost as these elements exert key controls on mountain slope stability (Haeberli and Whiteman, 2015). This section assesses knowledge gained since previous IPCC reports, in particular SREX (e.g., Seneviratne et al., 2012), and AR5 Working Group II (Cramer et al., 2014). In this section, observed and projected changes in hazards are covered first, followed by exposure, vulnerability and resulting impacts and risks, and finally disaster risk reduction and adaptation. Cryospheric hazards that constitute tipping points are also listed in Table 6.1 in Chapter 6.

Hazards assessed in this section range from localised effects on mountain slopes and adjacent valley floors (distance reach of up to several kilometres) to events reaching far into major valleys and even surrounding lowlands (reach of tens to hundreds of kilometres), and include cascading events. Changes in the cryosphere due to climate change influence the frequency and magnitude of hazards, the processes involved, and the locations exposed to the hazards (Figure 2.7). Natural hazards and associated disasters are sporadic by nature, and vulnerability and exposure exhibit strong geographic variations. Assessments of change are based not only on direct evidence, but also on laboratory experiments, theoretical considerations and calculations, and numerical modelling.

\subsubsection{Observed and Projected Changes}

\subsection{Unstable slopes, landslides and glacier instabilities}

Permafrost degradation and thaw as well as increased water flow into frozen slopes can increase the rate of movement of frozen debris bodies and lower their surface due to loss of ground ice (subsidence). Such processes affected engineered structures such as buildings, hazard protection structures, roads, or rail lines in all high mountains during recent decades (Section 2.3.4). Movement of frozen slopes and ground subsidence/heave are strongly related to ground temperature, ice content, and water input (Wirz et al., 2016; Kenner et al., 2017). Where massive ground ice gets exposed, retrogressive thaw erosion develops (Niu et al., 2012). The creep of rock glaciers (frozen debris tongues that slowly deform under gravity) is in principle expected to accelerate in response to rising ground temperatures, until substantial volumetric ice contents have melted out (Kääb et al., 2007; Arenson et al., 2015a). As documented for instance for sites in the European Alps and Scandinavia for recent years to decades, rock glaciers replenished debris flow starting zones at their fronts, so that the intensified material supply associated with accelerated movement (Section 2.2.4) contributed to increased debris flow activity (higher frequency, larger magnitudes) or slope destabilisation (Stoffel and Graf, 2015; Wirz et al., 2016; Kummert et al., 2017; Eriksen et al., 2018).

There is high confidence that the frequency of rocks detaching and falling from steep slopes (rock fall) has increased within zones of degrading permafrost over the past half-century, for instance in high mountains in North America, New Zealand, and Europe (Allen et al., 2011; Ravanel and Deline, 2011; Fischer et al., 2012; Coe et al., 2017). Compared to the SREX and AR5 reports, the confidence in this finding increased. Available field evidence agrees with theoretical considerations and calculations that permafrost thaw increases the likelihood of rock fall (and also rock avalanches, which have larger volumes compared to rock falls) (Gruber and Haeberli, 2007; Krautblatter et al., 2013). These conclusions are also supported by observed ice in the detachment zone of previous events in North America, Iceland and Europe (Geertsema et al., 2006; Phillips et al., 2017; Sæmundsson et al., 2018). Summer heat waves have in recent years triggered rock instability with delays of only a few days or weeks in the European Alps (Allen and Huggel, 2013; Ravanel et al., 2017). This is in line with theoretical considerations about fast thaw of ice filled frozen fractures in bedrock (Hasler et al., 2011) and other climate impacts on rock stability, such as from large temperature variations (Luethi et al., 2015). Similarly, permafrost thaw increased the frequency and volumes of landslides from frozen sediments in many mountain regions in recent decades (Wei et al., 2006; Ravanel et al., 2010; Lacelle et al., 2015). At lower elevations in the French Alps, though, climate driven changes such as a reduction in number of freezing days are projected to lead to a reduction in debris flows (Jomelli et al., 2009). 
A range of slope instability types was found to be connected to glacier retreat (Allen et al., 2011; Evans and Delaney, 2015). Debris left behind by retreating glaciers (moraines) slid or collapsed, or formed fast flowing water-debris mixtures (debris flows) in recent decades, for instance in the European and New Zealand Alps (Zimmermann and Haeberli, 1992; Blair, 1994; Curry et al., 2006; Eichel et al., 2018). Over decades to millennia, or even longer, rock slopes adjacent to or formerly covered by glaciers, became unstable and in some cases, eventually collapsed. Related landslide activity increased in recently deglacierised zones in most high mountains (Korup et al., 2012; McColl, 2012; Deline et al., 2015; Kos et al., 2016; Serrano et al., 2018). For example, according to Cloutier et al. (2017) more than two-thirds of the large landslides that occurred in Northern British Columbia between 1973-2003, occurred on cirque walls that have been exposed after glacier retreat from the mid-19th century on. Ice-rich permafrost environments following glacial retreat enhanced slope mass movements (Oliva and Ruiz-Fernández, 2015). At lower elevations, re-vegetation and rise of tree limit are able to stabilise shallow slope instabilities (Curry et al., 2006). Overall, there is high confidence that glacier retreat in general has in most high mountains destabilised adjacent debris and rock slopes over time scales from years to millennia, but robust statistics about current trends in this development are lacking. This finding reconfirms, and for some processes increases confidence in related findings from the SREX and AR5 reports.

Ice break-off and subsequent ice avalanches are natural processes at steep glacier fronts. How climate driven changes in geometry and thermal regime of such glaciers influenced ice avalanche hazards over years to decades depended strongly on local conditions, as shown for the European Alps (Fischer et al., 2013; Faillettaz et al., 2015). The few available observations are insufficient to detect trends. Where steep glaciers are frozen to bedrock, there is, however, medium evidence and high agreement from observations in the European Alps and from numerical simulations that failures of large parts of these glaciers were and will be facilitated in the future due to an increase in basal ice temperature (Fischer et al., 2013; Faillettaz et al., 2015; Gilbert et al., 2015).

In some regions, glacier surges constitute a recurring hazard, due to widespread, quasi-periodic and substantial increases in glacier speed over a period of a few months to years, often accompanied by glacier advance (Harrison et al., 2015; Sevestre and Benn, 2015). In a number of cases, mostly in North America and High Mountain Asia (Bevington and Copland, 2014; Round et al., 2017; Steiner et al., 2018), surge-related glacier advances dammed rivers, causing major floods. In rare cases, glacier surges directly inundated agricultural land and damaged infrastructure (Shangguan et al., 2016). Sevestre and Benn (2015) suggest that surging operates within a climatic envelope of temperature and precipitation conditions, and that shifts in these conditions can modify surge frequencies and magnitudes. Some glaciers have reduced or stopped surge activity, or are projected to do so within decades, as a consequence of negative glacier mass balances (Eisen et al., 2001; Kienholz et al., 2017). For such cases, related hazards can also be expected to decrease. In contrast, intensive or increased surge activity (Hewitt, 2007; Gardelle et al., 2012; Yasuda and Furuya, 2015) occurred in a region on and around the Western Tibetan plateau which exhibited balanced or even positive glacier mass budgets in recent decades (Brun et al., 2017). Enhanced melt water production was suggested to be able to trigger or enhance surge-type instability, in particular for glaciers that contain ice both at the melting point and considerably below (Dunse et al., 2015; Yasuda and Furuya, 2015; Nuth et al., 2019).

A rare type of glacier instability with large volumes (in the order of $10-100$ million $\mathrm{m}^{3}$ ) and high mobility (up to $200-300 \mathrm{~km} / \mathrm{h}$ ) results from the complete collapse of large sections of low-angle valley glaciers and subsequent combined ice/rock/debris avalanches. The largest of such glacier collapses have been reported in the Caucasus Mountains in 2002 (Kolka Glacier, 130 fatalities) (Huggel et al., 2005; Evans et al., 2009), and in the Aru Range in Tibet in 2016 (twin glacier collapses with 9 fatalities) (Kääb et al., 2018). Although there is no evidence that climate change has played a direct role in the 2002 event, changes in glacier mass balance, water input into the glaciers, and the frozen regime of the glacier beds were involved in the 2016 collapses and at least partly linked with climate change (Gilbert et al., 2018). Besides the 2016 Tibet cases, it is unknown if such massive and rare collapse-like glacier instabilities can be attributed to climate change.

\subsection{Snow avalanches}

Snow avalanches can occur either spontaneously due to meteorological factors such as loading by snowfall or liquid water infiltration following, for example, surface melt or rain-on-snow, or can be triggered by the passage of people in avalanche terrain, the impact of falling ice or rocks, or by explosives used for avalanche control (Schweizer et al., 2003). There is no published evidence found that addresses the links between climate change and accidental avalanches triggered by recreationists or workers. Changes in snow cover characteristics are expected to induce changes in spontaneous avalanche activity including changes in friction and flow regime (Naaim et al., 2013; Steinkogler et al., 2014).

Ballesteros-Cánovas et al. (2018) reported increased avalanche activity in some slopes of the Western Indian Himalaya over the past decades related to increased frequency of wet-snow conditions. In the European Alps, avalanche numbers and runout distance have decreased where snow depth decreased and air temperature increased (Teich et al., 2012; Eckert et al., 2013). In the European Alps and Tatras mountains, over past decades, there has been a decrease in avalanche mass and run-out distance and a decrease in avalanches with a powder part; avalanche numbers decreased below 2,000 m a.s.l., and increased above (Eckert et al., 2013; Lavigne et al., 2015; Gadek et al., 2017). A positive trend in the proportion of avalanches involving wet snow in December through February was shown for the last decades (Pielmeier et al., 2013; Naaim et al., 2016). Land use and land cover changes also contributed to changes in avalanches (García-Hernández et al., 2017; Giacona et al., 2018). Correlations between avalanche activity and the El Niño-Southern Oscillation (ENSO) were identified from 1950-2011 in North and South America but there was no significant temporal trend reported for avalanche activity (McClung, 2013). Mostly inconclusive results were reported by Sinickas et al. (2015) and Bellaire et al. (2016) regarding the 


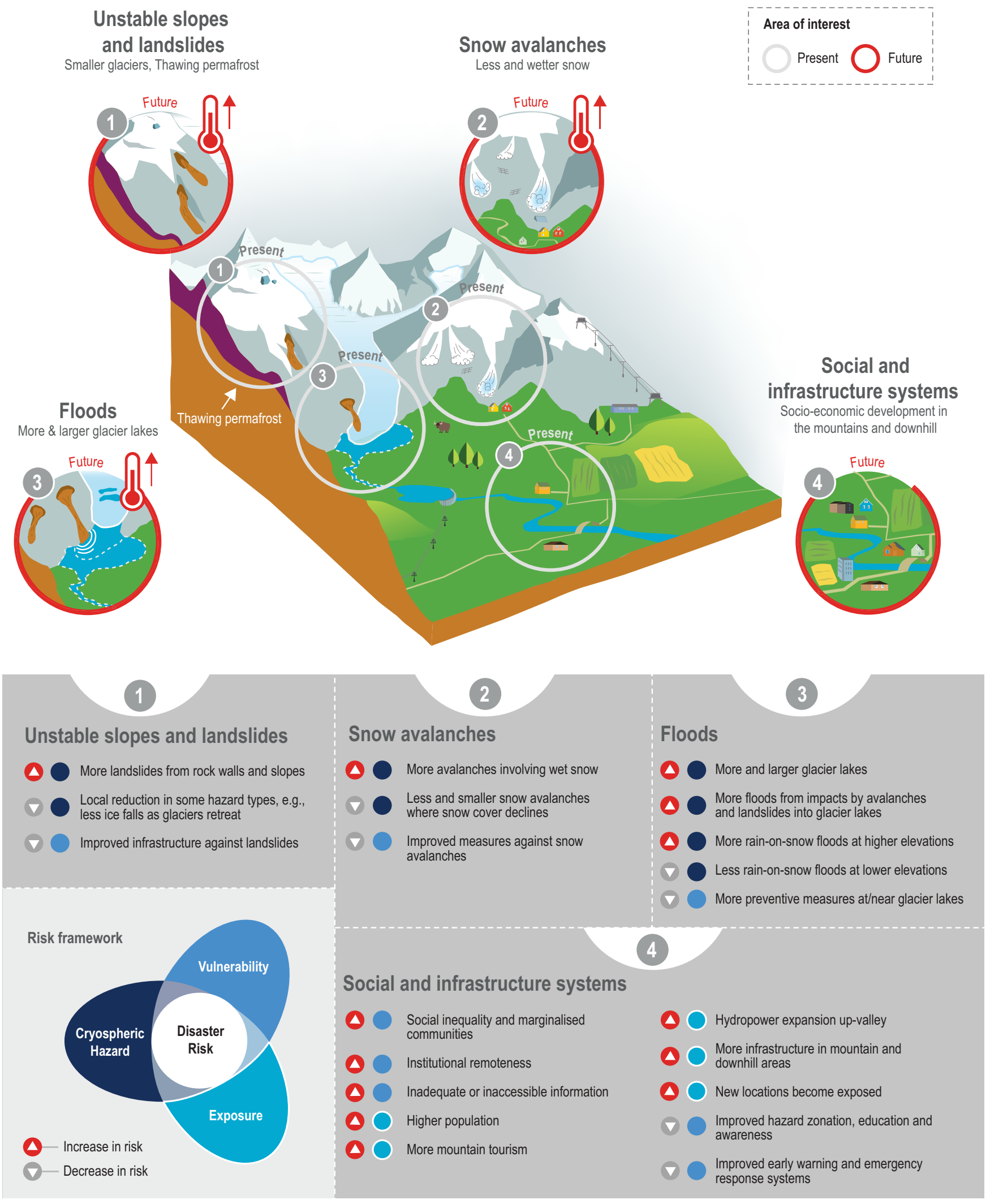

Figure 2.7 Anticipated changes in high mountain hazards under climate change, driven by changes in snow cover, glaciers and permafrost, overlay changes in the exposure and vulnerability of individuals, communities, and mountain infrastructure. 
relationship between avalanche activity, climate change and disaster risk reduction activities in North America. In summary, in particular in Europe, there is medium confidence in an increase in avalanche activity involving wet snow, and a decrease in the size and run-out distance of snow avalanches over the past decades.

Future projections mostly indicate an overall decrease in snow depth and snow cover duration at lower elevation (Section 2.2.2), but the probability of occurrence of occasionally large snow precipitation events is projected to remain possible throughout most of the $21 \mathrm{st}$ century (Section 2.2.1). Castebrunet et al. (2014) estimated an overall 20 and $30 \%$ decrease of natural avalanche activity in the French Alps for the mid and end of the 21st century, respectively, under A1B scenario, compared to the reference period 1960-1990. Katsuyama et al. (2017) reached similar conclusions for Northern Japan, and Lazar and Williams (2008) for North America. Avalanches involving wet snow are projected to occur more frequently during the winter at all elevations due to surface melt or rain-on-snow (e.g., Castebrunet et al., 2014, for the French Alps), and the overall number and runout distance of snow avalanches is projected to decrease in regions and elevations experiencing significant reduction in snow cover (Mock et al., 2017). In summary, there is medium evidence and high agreement that observed changes in avalanches in mountain regions will be exacerbated in the future, with generally a decrease in hazard at lower elevation, and mixed changes at higher elevation (increase in avalanches involving wet snow, no clear direction of trend for overall avalanche activity).

\subsection{Floods}

Glacier-related floods, including floods from lake outbursts (GLOFs), are documented for most glacierised mountain ranges and are among the most far-reaching glacier hazards. Past events affected areas tens to hundreds of kilometres downstream (Carrivick and Tweed, 2016). Retreating glaciers produced lakes at their fronts in many high mountain regions in recent decades (Frey et al., 2010; Gardelle et al., 2011; Loriaux and Casassa, 2013). Lake systems in High Mountain Asia also often developed on the surface of downwasting, low-slope glaciers where they coalesced from temporally variable supraglacial lakes (Benn et al., 2012; Narama et al., 2017). Corroborating SREX and AR5 findings, there is high confidence that current global glacier shrinkage caused new lakes to form and existing lakes to grow in most regions, for instance in South America, High mountain Asia and Europe (Loriaux and Casassa, 2013; Paul and Mölg, 2014; Zhang et al., 2015; Buckel et al., 2018). Exceptions occurred and are expected to occur in the future for few lakes where evaporation, runoff and reduced melt water influx in total led to a negative water balance (Sun et al., 2018a). Also, advancing glaciers temporarily dammed rivers, lake sections, or fjords (Stearns et al., 2015), for instance through surging (Round et al., 2017), causing particularly large floods once the ice dams breached. Outbursts from water bodies in and under glaciers are able to cause floods similar to those from surface lakes but little is known about the processes involved and any trends under climate change. In some cases, the glacier thermal regime played a role so that climate driven changes in thermal regime are expected to alter the hazard potential, depending on local conditions (Gilbert et al., 2012). Another source of large water bodies under glaciers and subsequent floods has been subglacial volcanic activity (Section 2.3.2.1.4). There is also high confidence that the number and area of glacier lakes will continue to increase in most regions in the coming decades, and new lakes will develop closer to steep and potentially unstable mountain walls where lake outbursts can be more easily triggered by the impact of landslides (Frey et al., 2010; ICIMOD, 2011; Allen et al., 2016a; Linsbauer et al., 2016; Colonia et al., 2017; Haeberli et al., 2017).

In contrast to the number and size of glacier lakes, trends in the number of glacier-related floods are not well known for recent decades (Carrivick and Tweed, 2016; Harrison et al., 2018), although a number of periods of increased and decreased flood activity have been documented for individual glaciers in North America and Greenland, spanning decades (Geertsema and Clague, 2005; Russell et al., 2011). A decrease in moraine-dammed glacier lake outburst floods in recent decades suggests a response of lake outburst activity being delayed by some decades with respect to glacier retreat (Harrison et al., 2018) but inventories might significantly underestimate the number of events (Veh et al., 2018). For the Himalaya, Veh et al. (2019)161 found no increase in the number of glacier lake outburst floods since the late 1980s. The degradation of permafrost and the melting of ice buried in lake dams have been shown to lower dam stability and contribute to outburst floods in many high mountain regions (Fujita et al., 2013; Erokhin et al., 2017; Narama et al., 2017).

Floods originating from the combination of rapidly melting snow and intense rainfall, referred to as rain-on-snow events, are some of the most damaging floods in mountain areas (Pomeroy et al., 2016; II Jeong and Sushama, 2018). The hydrological response of a catchment to a rain-on-snow event depends on the characteristics of the precipitation event, but also on turbulent fluxes driven by wind and humidity, which typically provide most of the melting energy during such events (Pomeroy et al., 2016), and the state of the snowpack, in particular the liquid water content (Würzer et al., 2016). An increase in the occurrence of rain-on-snow events in high-elevation zones, and a decrease at the lowest elevations have been reported (Western USA, 1949-2003, McCabe et al. (2007); Oregon, 1986-2010, Surfleet and Tullos (2013); Switzerland, 1972-2016, Moran-Tejéda et al. (2016), central Europe, 1950-2010, Freudiger et al. (2014)). These trends are consistent with studies carried out at the scale of the Northern Hemisphere (Putkonen and Roe, 2003; Ye et al., 2008; Cohen et al., 2015). There are no studies found on this topic in Africa and South America. In summary, evidence since AR5 suggests that rain-onsnow events have increased over the last decades at high elevations, particularly during transition periods from autumn to winter and winter to spring (medium confidence). The occurrence of rain-onsnow events has decreased over the last decade in low-elevation or low-latitude areas due to a decreasing duration of the snowpack, except for the coldest months of the year (medium confidence).

II Jeong and Sushama (2018) projected an increase in rain-onsnow events in winter and a decrease in spring, for the period 2041-2070 (RCP4.5 and RCP8.5) in North America, corroborated by Musselman et al. (2018). Their frequency in the Swiss Alps is projected to increase at elevations higher than 2,000 $\mathrm{m}$ a.s.l. (SRES A1B, 2025, 2055, and 2085) (Beniston and Stoffel, 2016). This study 
showed that the number of rain-on-snow events may increase by $50 \%$ with a regional temperature increase of $2^{\circ} \mathrm{C}$ to $4^{\circ} \mathrm{C}$, and decrease with a temperature rise exceeding $4^{\circ} \mathrm{C}$. In Alaska, an overall increase of rain-on-snow events is projected, however with a projected decline in the southwestern/southern region (Bieniek et al., 2018). In summary, evidence since AR5 suggests that the frequency of rain-on-snow events is projected to increase and occur earlier in spring and later in autumn at higher elevation and to decrease at lower elevation (high confidence).

\subsection{Combined hazards and cascading events}

The largest mountain disasters in terms of reach, damage and lives lost that involve ice, snow and permafrost occurred through a combination or chain of processes. New evidence since SREX and AR5 has strengthened these findings (Anacona et al., 2015a; Evans and Delaney, 2015). Some process chains occur frequently, while others are rare, specific to local circumstances and difficult to anticipate. Glacier lake outbursts were in many mountain regions and over recent decades documented to have been triggered by impact waves from snow-, ice- or rock-avalanches, landslides, iceberg calving events, or by temporary blockage of surface or subsurface drainage channels (Benn et al., 2012; Narama et al., 2017). Rock-slope instability and catastrophic failure along fjords caused tsunamis (Hermanns et al., 2014; Roberts et al., 2014). For instance, a landslide generated wave in 2015 at Taan Fjord, Alaska, ran up $193 \mathrm{~m}$ on the opposite slope and then travelled more than $20 \mathrm{~km}$ down the fjord (Higman et al., 2018). Earthquakes have been a starting point for different types of cascading events, for instance by causing snow-, ice- or rock-avalanches, and landslides (van der Woerd et al., 2004; Podolskiy et al., 2010; Cook and Butz, 2013; Sæmundsson et al., 2018). Glaciers and their moraines, including morainic lake dams, seem however, not particularly prone to earthquake triggered failure (Kargel et al., 2016).

Landslides and rock avalanches in glacier environments were often documented to entrain snow and ice that fluidise, and incorporate additional loose glacial sediments or water bodies, thereby multiplying their mobility, volume and reach (Schneider et al., 2011; Evans and Delaney, 2015). Rock avalanches onto glaciers triggered glacier advances in recent decades, for instance in North America, New Zealand and Europe, mainly through reducing surface melt (Deline, 2009; Reznichenko et al., 2011; Menounos et al., 2013). In glacier covered frozen rock walls, particularly complex thermal, mechanical, hydraulic and hydrologic interactions between steep glaciers, frozen rock and its ice content, and unfrozen rock sections lead to combined rock/ice instabilities that are difficult to observe and anticipate (Harris et al., 2009; Fischer et al., 2013; Ravanel et al., 2017). There is limited evidence of observed direct event chains to project future trends. However, from the observed and projected degradation of permafrost, shrinkage of glaciers and increase in glacier lakes it is reasonable to assume that event chains involving these could increase in frequency or magnitude, and that accordingly hazard zones could expand.
Volcanoes covered by snow and ice often produce substantial melt water during eruptions. This typically results in floods and/or lahars (mixtures of melt water and volcanic debris) which can be exceptionally violent and cause large-scale loss of life and destruction to infrastructure (Barr et al., 2018). The most devastating example from recent history occurred in 1985, when the medium-sized eruption of Nevado del Ruiz volcano, Colombia, produced lahars that killed more than 23,000 people some $70 \mathrm{~km}$ downstream (Pierson et al., 1990). Hazards associated with ice and snow-clad volcanoes have been reported mostly from the Cordilleras of the Americas, but also from the Aleutian arc (USA), Mexico, Kamchatka (Russia), Japan, New Zealand and Iceland (Seynova et al., 2017). In particular, under Icelandic glaciers, volcanic activity and eruptions melted large amounts of ice and caused especially large floods if water accumulated underneath the glacier (Björnsson, 2003; Seneviratne et al., 2012). There is medium confidence that the overall hazard related to floods and lahars from ice- and snow-clad volcanoes will gradually diminish over years-to-decades as glaciers and seasonal snow cover continue to decrease under climate change (Aguilera et al., 2004; Barr et al., 2018). On the other hand, shrinkage of glaciers may uncover steep slopes of unconsolidated volcanic sediments, thus decreasing in the future the resistance of these volcano flanks to heavy rain fall and increasing the hazard from related debris flows (Vallance, 2005). In summary, future changes in snow and ice are expected to modify the impacts of volcanic activity of snow- and ice-clad volcanoes (high confidence) although in complex and locally variable ways and at a variety of time scales (Barr et al., 2018; Swindles et al., 2018).

\subsubsection{Exposure, Vulnerability and Impacts}

\subsection{Changes in exposure}

Confirming findings from SREX, there is high confidence that the exposure of people and infrastructure to cryosphere hazards in high mountain regions has increased over recent decades, and this trend is expected to continue in the future (Figure 2.7). In some regions, tourism development has increased exposure, where often weakly regulated expansion of infrastructure such as roads, trails, and overnight lodging brought more visitors into remote valleys and exposed sites (Gardner et al., 2002; Uniyal, 2013). As an example for the consequences of increased exposure, many of the more than 350 fatalities resulting from the 2015 earthquake triggered snow-ice avalanche in Langtang, Nepal, were foreign trekkers and their local guides (Kargel et al., 2016). Further, several thousand religious pilgrims were killed during the 2013 Kedarnath glacier flood disaster (State of Uttarakhand, Northern India) (Kala, 2014). The expansion of hydropower (Section 2.3.1) is another key factor, and in the Himalaya alone, up to two-thirds of the current and planned hydropower projects are located in the path of potential glacier floods (Schwanghart et al., 2016). Changes in exposure of local communities, for instance, through emigration driven by climate change related threats (Grau and Aide, 2007; Gosai and Sulewski, 2014), or increased connectivity and quality of life in urban centres (Tiwari and Joshi, 2015), are complex and vary regionally. The effects of changes in exposure on labour migration and relocation of entire communities are discussed in Section 2.3.7. 


\subsection{Changes in vulnerability}

Considering the wide ranging social, economic, and institutional factors that enable communities to adequately prepare for, respond to and recover from climate change impacts (Cutter and Morath, 2013), there is limited evidence and high agreement that mountain communities, particularly within developing countries, are highly vulnerable to the adverse effects of enhanced cryosphere hazards. There are few studies that have systematically investigated the vulnerability of mountain communities to natural hazards (Carey et al., 2017). Coping capacities to withstand impacts from natural hazards in mountain communities are constrained due to a number of reasons. Fundamental weather and climate information is lacking to support both short-term early warning for imminent disasters, and long-term adaptation planning (Rohrer et al., 2013; Xenarios et al., 2018). Communities may be politically and socially marginalised (Marston, 2008). Incomes are typically lower and opportunities for livelihood diversification restricted (McDowell et al., 2013). Emergency responders can have difficulties accessing remote mountain valleys after disasters strike (Sati and Gahalaut, 2013). Cultural or social ties to the land can limit freedom of movement (Oliver-Smith, 1996). Conversely, there is evidence that some mountain communities exhibit enhanced levels of resilience, drawing on long-standing experience, and Indigenous knowledge and local knowledge (Cross-Chapter Box 4 in Chapter 1) gained over many centuries of living with extremes of climate and related disasters (Gardner and Dekens, 2006). In the absence of sufficient data, few studies have considered temporal trends in vulnerability (Huggel et al., 2015a).

\subsection{Impacts on livelihoods}

Empirical evidence from past events shows that cryosphere related landslides and floods can have severe impacts on lives and livelihoods, often extending far beyond the directly affected region, and persisting for several years. Glacier lake outburst floods alone have over the past two centuries directly caused at least 400 deaths in Europe, 5,745 deaths in South America, and 6,300 deaths in Asia (Carrivick and Tweed, 2016), although these numbers are heavily skewed by individual large events occurring in Huaraz and Yungay, Peru (Carey, 2005) and Kedarnath, India (Allen et al., 2016b).

Economic losses associated with these events are incurred through two pathways. The first consists of direct losses due to the disasters, and the second includes indirect costs from the additional risk and loss of potential opportunities, or from additional investment that would be necessary to manage or adapt to the challenges brought about by the cryosphere changes. Nationwide economic impacts from glacier floods have been greatest in Nepal and Bhutan (Carrivick and Tweed, 2016). The disruption of vital transportation corridors that can impact trading of goods and services (Gupta and Sah, 2008; Khanal et al., 2015), and the loss of earnings from tourism can represent significant far-reaching and long-lasting impacts (Nothiger and Elsasser, 2004; IHCAP, 2017). The Dig Tsho flood in the Khumbu Himal of Nepal in 1985 damaged a hydropower plant and other properties, with estimated economic losses of 500 million USD (Shrestha et al., 2010). Less tangible, but equally important impacts concern the cultural and social disruption resulting from temporary or permanent evacuation (Oliver-Smith, 1979). According to the International Disaster - Emergency Events Database (EM-DAT), over the period 1985-2014, absolute economic losses in mountain regions from all flood and mass movements (including non-cryosphere origins) were highest in the Hindu Kush Himalaya region (45 billion USD), followed by the European Alps (7 billion USD), and the Andes (3 billion USD) (Stäubli et al., 2018). For example, a project to dig a channel in Tsho Rolpa glacier in Nepal that lowered a glacial lake cost 3 million USD in 2000 (Bajracharya, 2010), and similar measures have been taken at Imja Tsho Lake in Nepal in 2016 (Cuellar and McKinney, 2017). Other impacts are related to drinking and irrigation water and livelihoods (Section 2.3.1). In summary, there is high confidence that in the context of mountain flood and landslide hazards, exposure, and vulnerability growing in the coming century, significant risk reduction and adaptation strategies will be required to avoid increased impacts.

\subsubsection{Disaster Risk Reduction and Adaptation}

There is medium confidence that applying an integrative socioecological risk perspective to flood, avalanche and landslide hazards in high mountain regions paves the way for adaptation strategies that can best address the underlying components of hazard, exposure and vulnerability (Carey et al., 2014; McDowell and Koppes, 2017; Allen et al., 2018; Vaidya et al., 2019). Some degree of adaptation action has been identified in a number of countries with glacier covered mountain ranges, mostly in the form of reactive responses (rather than formal anticipatory plans) to high mountain hazards (Xenarios et al., 2018; McDowell et al., 2019) (Figure 2.9). However, scientific literature reflecting on lessons learned from adaptation efforts generally remains scarce. Specifically for flood and landslide hazards, adaptation strategies that were applied include: hard engineering solutions such as lowering of glacier lake levels, channel engineering, or slope stabilisation that reduce the hazard potential; nature-based solutions such as revegetation efforts to stabilise hazard prone slopes or channels; hazard and risk mapping as a basis for land zoning and early warning systems that reduce potential exposure; various community level interventions to develop disaster response programmes, build local capacities and reduce vulnerability. For example, there is a long tradition of engineered responses to reduce glacier flood risk, most notably beginning in the mid-20th century in Peru (Box 2.4), Italian and Swiss Alps (Haeberli et al., 2001), and more recently in the Himalaya (Ives et al., 2010). There is no published evidence that avalanche risk management, through defence structures design and norms, control measures and warning systems, has been modified as an adaptation to climate change, over the past decades. Projected changes in avalanche character bear potential reductions of the effectiveness of current approaches for infrastructure design and avalanche risk management (Ancey and Bain, 2015).

Early warning systems necessitate strong local engagement and capacity building to ensure communities know how to prepare for and respond to emergencies, and to ensure the long-term sustainability of any such project. In Pakistan and Chile, for instance, glacier flood warnings, evacuation and post-disaster relief have largely been community led (Ashraf et al., 2012; Anacona et al., 2015b). 
Cutter et al. (2012) highlight the post-recovery and reconstruction period as an opportunity to build new resilience and adaptive capacities. Ziegler et al. (2014) exemplify consequences when such process is rushed or poorly supported by appropriate long-term planning, as illustrated following the 2013 Kedarnath glacier flood disaster, where guest houses and even schools were being rebuilt in the same exposed locations, driven by short-term perspectives. As changes in the mountain cryosphere, together with socioeconomic, cultural and political developments are producing conditions beyond historical precedent, related responses are suggested to include forward-thinking planning and anticipation of emerging risks and opportunities (Haeberli et al., 2016).

Researchers, policymakers, international donors and local communities do not always agree on the timing of disaster risk reduction projects and programs, impeding full coordination (Huggel et al., 2015b; Allen et al., 2018). Several authors highlight the value of improved evidential basis to underpin adaptation planning. Thereby, transdisciplinary and cross-regional collaboration that places human societies at the centre of studies provides a basis for more effective and sustainable adaptation strategies (McDowell et al., 2014; Carey et al., 2017; McDowell et al., 2019; Vaidya et al., 2019).

In summary, the evidence from regions affected by cryospheric floods, avalanches and landslides generally confirms the findings from the SREX report (Chapter 3), including the requirement for multi-pronged approaches customised to local circumstances, integration of Indigenous knowledge and local knowledge (Cross-Chapter Box 4 in Chapter 1) together with improved scientific understanding and technical capacities, strong local participation and early engagement in the process, and high-level communication and exchange between all actors. Particularly for mountain regions, there is high confidence that integration of knowledge and practices across natural and social sciences, and the humanities, is most efficient in addressing complex hazards and risks related to glaciers, snow, and permafrost.

\section{Box 2.4 | Challenges to Farmers and Local Population Related to Shrinkages in the Cryosphere: Cordillera Blanca, Peru}

The Cordillera Blanca of Peru contains most of the glaciers in the tropics, and its glacier coverage declined significantly in the recent past (Burns and Nolin, 2014; Mark et al., 2017). Since the 1940s, glacier hazards have killed thousands (Carey, 2005) and remain threatening. Glacier wastage has also reduced river runoff in most of its basins in recent decades, particularly in the dry season (Baraer et al., 2012; Vuille et al., 2018). Residents living adjacent to the Cordillera Blanca have long recognised this glacier shrinkage, including rural populations living near glaciers and urban residents worried about glacier lake floods and glacier landslides (Jurt et al., 2015; Walter, 2017). Glacier hazards and the glacier runoff variability increase exposure and uncertainty while diminishing adaptive capacity (Rasmussen, 2016).

Cordillera Blanca residents' risk of glacier-related disasters is amplified by intersecting physical and societal factors. Cryosphere hazards include expanding or newly forming glacial lakes, slope instability, and other consequences of rising temperatures, and precipitation changes (Emmer et al., 2016; Colonia et al., 2017; Haeberli et al., 2017). Human vulnerability to these hazards is conditioned by factors such as poverty, limited political influence and resources, minimal access to education and healthcare, and weak government institutions (Hegglin and Huggel, 2008; Carey et al., 2012; Lynch, 2012; Carey et al., 2014; Heikkinen, 2017). Early warning systems have been, or are being, installed at glacial lakes Laguna 513 and Palcacocha to protect populations (Muñoz et al., 2016). Laguna 513 was lowered by $20 \mathrm{~m}$ for outburst prevention in the early 1990s but nonetheless caused a destructive flood in 2010, though much smaller and less destructive than a flood that would have been expected without previous lake mitigation works (Carey et al., 2012; Schneider et al., 2014). An early warning system was subsequently installed, but some local residents destroyed it in 2017 due to political, social and cultural conflicts (Fraser, 2017). The nearby Lake Palcacocha also threatens populations (Wegner, 2014; Somos-Valenzuela et al., 2016). The usefulness for ground-level education and communication regarding advanced early warning systems has been demonstrated in Peru (Muñoz et al., 2016).

Vulnerability to hydrologic variability and declining glacier runoff is also shaped by intertwining human and biophysical drivers playing out in dynamic hydro-social systems (Bury et al., 2013; Rasmussen 2016; Drenkhan et al., 2015; Carey et al., 2017). Water security is influenced by both water availability (supply from glaciers) as well as by water distribution, which is affected by factors such as water laws and policies, global demand for agricultural products grown in the lower Santa River basin, energy demands and hydroelectricity production, potable water usage, and livelihood transformations over time (Carey et al., 2014; Vuille et al., 2018). In some cases, the formation of new glacial lakes can create opportunities as well as hazards, such as new tourist attractions and reservoirs of water, thereby showing how socioeconomic and geophysical forces intersect in complex ways (Colonia et al., 2017). 


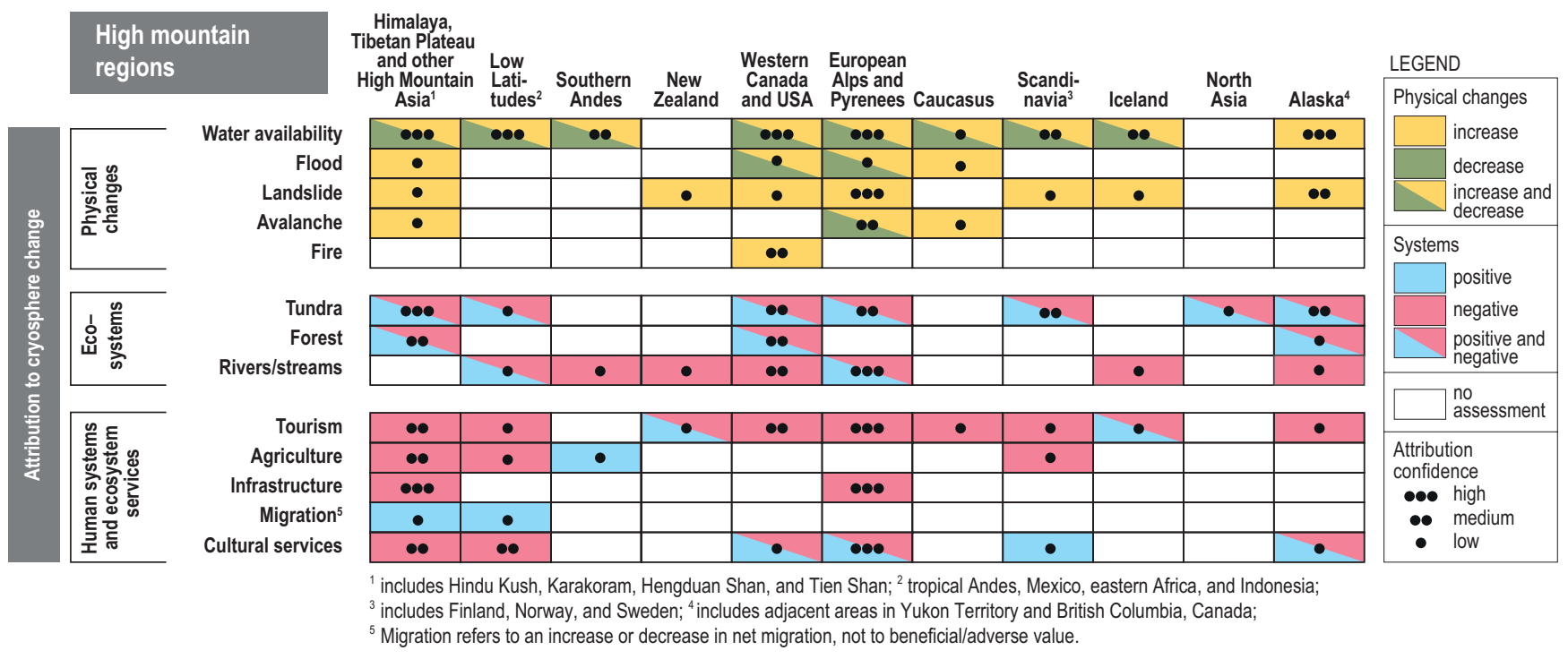

Figure 2.8 Synthesis of observed physical changes and impacts on ecosystems and human systems and ecosystems services in eleven high mountain regions over past decades that can at least partly be attributed to changes in the cryosphere. Only observations documented in the scientific literature are shown, but impacts may also be experienced elsewhere. For physical changes yellow/green refers to an increase/decrease, respectively, in amount or frequency of the measured variable. For impacts on ecosystems and human systems and ecosystems services blue or red depicts whether an observed impact is positive (beneficial) or negative (adverse). Cells assigned 'increase and decrease' indicate that within that region both increase and decrease of physical changes are found, but are not necessarily equal; the same holds for cells showing 'positive and negative' impacts. Confidence levels refer to confidence in attribution to cryospheric changes. No assessment means: not applicable, not assessed at regional scale, or the evidence is insufficient for assessment. Tundra refers to tundra and alpine meadows. Migration refers to an increase and decrease in net migration, not beneficial/adverse value. Impacts on tourism refer to the operating conditions for the tourism sector. Cultural services include cultural identity, sense of home, intrinsic and aesthetic values, as well as contributions from glacier archaeology. Figure is based on observed impacts listed in Table SM2.11.

\subsubsection{Ecosystems}

Widespread climate driven ecological changes have occurred in high mountain ecosystems over the past century. Those impacts were assessed in a dedicated manner only in earlier IPCC assessments (Beniston and Fox, 1996; Gitay et al., 2001; Fischlin et al., 2007) but not in AR5 (Settele et al., 2014). Two of the most evident changes include range shifts of plants and animals in Central Europe and the Himalaya but also for other mountain regions (e.g., Morueta-Holme et al., 2015; Evangelista et al., 2016; Freeman et al., 2018; Liang et al., 2018; You et al., 2018; He et al., 2019), and increases in species richness on mountain summits (Khamis et al., 2016; Fell et al., 2017; Steinbauer et al., 2018) of which some have accelerated during recent decades (e.g., Steinbauer et al., 2018), though slowing over the past ten years in Austria (e.g., Lamprecht et al., 2018). While many changes in freshwater communities have been directly attributed to changes in the cryosphere (Jacobsen et al., 2012; Milner et al., 2017), separating the direct influence of atmospheric warming from the influence of concomitant cryospheric change and independent biotic processes has been often challenging for terrestrial ecosystems (Grytnes et al., 2014; Lesica and Crone, 2016; Frei et al., 2018; Lamprecht et al., 2018). Changing climate in high mountains places further stress on biota, which are already impacted by land use and its change, direct exploitation, and pollutants (Díaz et al., 2019; Wester et al., 2019). Species are required to shift their behaviours, including seasonal aspects, and distributional ranges to track suitable climate conditions (Settele et al., 2014). In the Special Report on Global Warming of $1.5^{\circ} \mathrm{C}$ (SR15), climate change scenarios exceeding mean global warming of $1.5^{\circ} \mathrm{C}$ relative to preindustrial levels have been estimated to lead to major impacts on species abundances, community structure, and ecosystem functioning in high mountain areas (Hoegh-Guldberg et al., 2018). The size and isolation of mountain habitats (Steinbauer et al., 2016; Cotto et al., 2017), which may vary strongly with the topography of mountain ridges (Elsen and Tingley, 2015; Graae et al., 2018), affects critically the survival of species as they migrate across mountain ranges, increasing in general the risks for many species from climate change (Settele et al., 2014; Dobrowski and Parks, 2016).

\subsubsection{Terrestrial Biota}

The cryosphere can play a critical role in moderating and driving how species respond to climate change in high mountains (high confidence). Many mountain plant and animal species have changed abundances and migrated upslope while expanding or contracting their ranges over the past decades to century, whereas others show no change (Morueta-Holme et al., 2015; Suding et al., 2015; Lesica and Crone, 2016; Fadrique et al., 2018; Freeman et al., 2018; Rumpf et al., 2018; Johnston et al., 2019; Rumpf et al., 2019) (medium agreement, robust evidence). These responses are often linked directly to warming, yet a changing cryosphere, for example, in the form of decreasing snow thickness or altered seasonality of snow (e.g., Matteodo et al., 2016; Kirkpatrick et al., 2017; Amagai et al., 2018; Wu et al., 2018) or indirectly leading to changes in soil moisture (Harpold and Molotch, 2015), can play a significant role for growth, fitness and survival of many species (e.g., Grytnes et al., 2014; Winkler et al., 2016) (medium evidence, high agreement).

Cryospheric changes were found to be beneficial for some plant species and for ecosystems in some regions, improving a number of 
ecosystem services, such as by provisioning new habitat for endemic plant species and increasing plant productivity (high confidence). Decreasing snow cover duration, glacier retreat and permafrost thaw have already and will over coming decades allow plant species, including some endemic species, to increase their abundance and extend their range in many mountain ranges (Yang et al., 2010a; Grytnes et al., 2014; Elsen and Tingley, 2015; Dolezal et al., 2016; Wang et al., 2016b; D'Amico et al., 2017; Liang et al., 2018; Yang et al., 2018; You et al., 2018; He et al., 2019). Over recent decades, plant colonisation after glacier retreat has been swift, for example, at many sites with favourable soils in the European Alps (Matthews and Vater, 2015; Fickert and Grüninger, 2018) or has even accelerated compared to 100 years ago (Fickert et al., 2016). At other sites of the European Alps (D'Amico et al., 2017) and in other mountain ranges (e.g., Andes and Alaska; Darcy et al., 2018; Zimmer et al., 2018) the rate of colonisation remains slow due to soil type, soil formation and phosphorous limitation (Darcy et al., 2018). In Bhutan, snowlines have ascended and new plant species have established themselves in these areas, yet despite range expansion and increased productivity, yak herders describe impacts on the ecosystem services as mostly negative (Wangchuk and Wangdi, 2018). Earlier snowmelt often leads to earlier plant growth and, provided there is sufficient water, including from underlying permafrost, plant productivity has increased in many alpine regions (e.g., Williams et al., 2015; Yang et al., 2018). Decreased snow cover duration has led to colonisation of snowbed communities by wide-ranging species in several regions, for example, in the Australian Alps (Pickering et al., 2014), though this can lead to declines in the abundance of resident species, for example, in the Swiss Alps (Matteodo et al., 2016).

Cryospheric change in high mountains directly harms some plant species and ecosystems in some regions, degrading a number of ecosystem services, such as maintaining regional and global biodiversity, and some provisioning services, for example, fodder or wood production, in terms of timing and magnitude (high confidence). In mountains, microrefugia (a local environment different from surrounding areas) and isolation have contributed to high plant endemism that increases with elevation (Steinbauer et al., 2016; Zhang and Zhang, 2017; Muellner-Riehl, 2019). Microrefugia may enable alpine species to persist if global warming remains below $2^{\circ} \mathrm{C}$ relative pre-industrial levels (Scherrer and Körner, 2011; Hannah et al., 2014; Graae et al., 2018) (medium evidence, medium agreement). Yet, where glaciers have been retreating over recent decades, cool microrefugia have shifted location or decreased in extent (Gentili et al., 2015). In regions with insufficient summer precipitation, earlier snowmelt and absence of permafrost lead to insufficient water supply during the growing season, and consequently an earlier end of peak season, altered species composition, and a decline in greenness or productivity (Trujillo et al., 2012; Sloat et al., 2015; Williams et al., 2015; Yang et al., 2018) (medium evidence, high agreement). Across elevations, alpine-restricted species show greater sensitivity to the timing of snowmelt than wide ranging species (Lesica, 2014; Winkler et al., 2018), and though the cause is often not known, some alpine-restricted species have declined in abundance or disappeared in regions with distinctive flora (Evangelista et al., 2016; Giménez-Benavides et al., 2018; Lamprecht et al., 2018; Panetta et al., 2018) (medium evidence, high agreement).

The shrinking cryosphere represents a loss of critical habitat for wildlife that depend on snow and ice cover, affecting well-known and unique high-elevation species (high confidence). Areas with seasonal snow and glaciers are essential habitat for birds and mammals within mountain ecosystems for foraging, relief from climate stress, food caching and nesting grounds (Hall et al., 2016; Rosvold, 2016) (robust evidence). Above 5,000 m a.s.l. in Peru, there was recently a first observation of bird nesting for which its nesting may be glacier obligate (Hardy et al., 2018). The insulated and thermally stable region under the snow at the soil-snow interface, termed the subnivean, has been affected by changing snowpack, limiting winter activity and decreasing population growth for some mountain animals, including frogs, rodents and small carnivores (Penczykowski et al., 2017; Zuckerberg and Pauli, 2018; Kissel et al., 2019) (medium evidence). Many mountain animals have been observed to change their behaviour in a subtle manner, for example., in foraging or hunting behaviour, due to cryospheric changes (e.g., Rosvold, 2016; Büntgen et al., 2017; Mahoney et al., 2018) (medium evidence, high agreement). In the Canadian Rocky Mountains, grizzly bears have moved to new snow free habitat after emerging in spring from hibernation to dig for forage, which may increase the risk of human-bear encounters (Berman et al., 2019). In the US Central Rocky Mountains, migratory herbivores, such as elk, moose and bison, track newly emergent vegetation that greens soon after snowmelt (Merkle et al., 2016). For elk, this was found to increase fat gain (Middleton et al., 2018). Due to loss of snow patches that increase surface water and thus insect abundance, some mammal species, for example, reindeer and ibex, have changed their foraging behaviour to evade the biting insects with negative impacts on reproductive fitness (Vors and Boyce, 2009; Büntgen et al., 2017).

Many endemic plant and animal species including mammals and invertebrates in high mountain regions are vulnerable to further decreasing snow cover duration, such as later onset of snow accumulation and/or earlier snowmelt (high confidence) (Williams et al., 2015; Slatyer et al., 2017). Winter-white animals for which coat or plumage colour is cued by day length will confront more days with brown snowless ground (Zimova et al., 2018), which has already contributed to range contractions for several species, including hares and ptarmigan (Imperio et al., 2013; Sultaire et al., 2016; Pedersen et al., 2017) (robust evidence). Under all climate scenarios, the duration of this camouflage mismatch will increase, enhancing predation rates thereby decreasing populations of coat-colour changing species (e.g., 24\% decrease by late century under RCP8.5 for snowshoe hares; Zimova et al., 2016; see also Atmeh et al., 2018) (medium evidence, high agreement). For roe deer (Plard et al., 2014) and mountain goats (White et al., 2017), climate driven changes in snowmelt duration and summer temperatures will reduce survival considerably under RCP4.5 and RCP8.5 scenarios (medium evidence, high agreement). 


\subsubsection{Freshwater Biota}

Biota in mountain freshwater ecosystems is affected by cryospheric change through alterations in both the quantity and timing of runoff from glaciers and snowmelt. Where melt water from glaciers decreases, river flows have become more variable, with water temperature and overall channel stability increasing and habitats becoming less complex (Giersch et al., 2017; Milner et al., 2017) (medium evidence, medium agreement).

Analysis of three invertebrate datasets from tropical (Ecuador), temperate (Italian Alps) and sub-Arctic (Iceland) alpine regions indicates that a number of cold-adapted species have decreased in abundance below a threshold of watershed glacier cover varying from $19-32 \%$. With complete loss of the glaciers, $11-38 \%$ of the regional species will be lost (Jacobsen et al., 2012; Milner et al., 2017) (medium confidence). As evidenced in Europe (Pyrenees, Italian Alps) and North America (Rocky Mountains) (Brown et al., 2007; Giersch et al., 2015; Giersch et al., 2017; Lencioni, 2018) the loss of these invertebrates - many of them endemic - as glacier runoff decreases and transitions to a regime more dominated by snowmelt leading to a reduction in turnover between and within stream reaches (beta diversity) and regional (gamma) diversity (very high confidence). Regional genetic diversity within individual riverine invertebrate species in mountain headwater areas has decreased with the loss of environmental heterogeneity (Giersch et al., 2017), as decreasing glacier runoff reduces the isolation of individuals permitting a greater degree of genetic intermixing (Finn et al., 2013; Finn et al., 2016; Jordan et al., 2016; Hotaling et al., 2018) (medium evidence, high agreement). However, local (alpha) diversity, dominated by generalist species of invertebrates and algae, has increased (Khamis et al., 2016; Fell et al., 2017; Brown et al., 2018) (very high confidence) in certain regions as species move upstream, although not in the Andes, where downstream migration has been observed (Jacobsen et al., 2014; Cauvy-Fraunié et al., 2016).

Many climate variables influence fisheries, through both direct and indirect pathways. The key variables linked to cryospheric change include: changes in air and water temperature, precipitation, nutrient levels and ice cover (Stenseth et al., 2003). A shrinking cryosphere has significantly affected cold mountain resident salmonids (e.g., brook trout, Salvelinus fontinalis), causing further migration upstream in summer thereby shrinking their range (Hari et al., 2006; Eby et al., 2014; Young et al., 2018). Within the Yanamarey watershed of the Cordillera Blanca in Peru, fish stocks have either declined markedly or have become extinct in many streams, possibly due to seasonal reductions of fish habitat in the upper watershed resulting from glacier recession (Bury et al., 2011; Vuille et al., 2018). In contrast, glacier recession in the mountains of coastal Alaska and to a lesser extent the Pacific northwest have created a large number of new stream systems that have been, and could continue to be with further glacier retreat, colonised from the sea by salmon species that contribute to both commercial and sport fisheries (Milner et al., 2017; Schoen et al., 2017) (medium confidence). Changes in water temperature will vary seasonally, and a potential decreased frequency of rain-on-snow events in winter compared to rain-on-ground would increase water temperature, benefiting overwintering survival (Leach and Moore, 2014). Increased water temperature remaining below thermal tolerance limits for fish and occurring earlier in the year can benefit overall fish growth and increase fitness (Comola et al., 2015) (medium evidence, medium agreement).

In the future, increased primary production dominated by diatoms and golden algae will occur in streams as glacier runoff decreases, although some cold-tolerant diatom species will be lost, resulting in a decrease in regional diversity (Fell et al., 2017; Fell et al., 2018). Reduced glacier runoff is projected to improve water clarity in many mountain lakes, increasing biotic diversity and the abundance of bacterial and algal communities and thus primary production (Peter and Sommaruga, 2016) (limited evidence). Extinction of range-restricted prey species may increase as more favourable conditions facilitate the upstream movement of large bodied invertebrate predators (Khamis et al., 2015) (medium confidence). Modelling studies indicate a reduction in the range of native species, notably trout, in mountain streams, (Papadaki et al., 2016; Vigano et al., 2016; Young et al., 2018) (medium evidence, high agreement), which will potentially impact sport fisheries. In northwest North America, where salmon are important in native subsistence as well as commercial and sport fisheries, all species will potentially be affected by reductions in glacial runoff from mountain glaciers over time (Milner et al., 2017; Schoen et al., 2017), particularly in larger systems where migratory corridors to spawning grounds are reduced (medium confidence).

In summary, cryospheric change will alter freshwater communities with increases in local biodiversity but range shrinkage and extinctions for some species causes regional biodiversity to decrease (robust evidence, medium agreement, i.e., high confidence).

\subsubsection{Ecosystem Services and Adaptation}

The trend to a higher productivity in high mountain ecosystems due to a warmer environment and cryospheric changes, affects provisioning and regulating services (high confidence). Due to earlier snowmelt, the growing season has begun earlier, for example, on the Tibetan Plateau, and in the Swiss Alps (Wang et al., 2017; Xie et al., 2018), and in some regions earlier growth has been linked to greater plant production or greater net ecosystem production, possibly affecting carbon uptake (Scholz et al., 2018; Wang et al., 2018; Wu et al., 2018). In other areas productivity has decreased, despite a longer growing season, for example, in the US Rocky Mountains, US Sierra Nevada Mountains, Swiss Alps, and Tibetan Plateau (Arnold et al., 2014; Sloat et al., 2015; Wang et al., 2017; De Boeck et al., 2018; Knowles et al., 2018) (robust evidence, medium agreement). Changed productivity of the vegetation in turn can affect the timing, quantity and quality of water supply, a critical regulating service ecosystems play in high mountain areas (Goulden and Bales, 2014; Hubbard et al., 2018) (medium confidence). Permafrost degradation has dramatically changed some alpine ecosystems through altered soil temperature and permeability, decreasing the climate regulating service of a vast region and leading to lowered ground water and new and shrinking lakes on the Tibetan Plateau (Jin et al., 2009; Yang et al., 2010b; Shen et al., 2018) (medium evidence, high agreement). 
Ecosystems and their services are vulnerable to changes in the intensity and/or the frequency of an ecological disturbance that exceed the previous range of variation (Johnstone et al., 2016; Camac et al., 2017; Fairman et al., 2017); cf. 3.4.3.2 Ecosystems and their Services) (high confidence). For example, in the Western USA, mountain ecosystems are experiencing an increase in the number and extent of wildfires, which have been attributed to many factors, including climate factors such as earlier snowmelt and vapour-pressure deficit (Settele et al., 2014; Westerling, 2016; Kitzberger et al., 2017; Littell, 2018; Littell et al., 2018). Similarly, landslides and floods in many areas have been attributed to cryospheric changes (Section 2.3.2). Disturbances can feedback and diminish many of the ecosystem services such as provisioning, regulating and cultural services (Millar and Stephenson, 2015; McDowell and Koppes, 2017; Mcdowell et al., 2018; Murphy et al., 2018; Maxwell et al., 2019). Consistent with AR5 findings (Settele et al., 2014) the capacity of many freshwater and terrestrial mountain species to adapt naturally to climate change is projected to be exceeded for high warming levels, leading to species migration across mountain ranges or loss with consequences for many ecosystem services (Elsen and Tingley, 2015; Dobrowski and Parks, 2016; Pecl et al., 2017; Rumpf et al., 2019) (robust evidence, medium agreement, i.e., high confidence). Although the adaptive potential of aquatic biota to projected changes in glacial runoff is not fully understood (Lencioni et al., 2015), dispersion and phenotypic plasticity together with additional microrefugia formation due to cryospheric changes, is expected to help threatened species to better adapt, perhaps even in the long term (Shama and Robinson, 2009). Likewise, traits shaped by climate and with high genetically-based standing variation may be used to spatially identify, map and manage global 'hotspots' for evolutionary rescue from climate change (Jones et al., 2018; Mills et al., 2018). Nature conservation increases the potential for mitigating adverse effects on many of these ecosystem services, including those that are essential for the support of the livelihoods and the culture of mountain peoples, including economical aspects such as recreation and tourism (e.g., Palomo, 2017; Elsen et al., 2018; Wester et al., 2019) (medium confidence).

\subsubsection{Infrastructure and Mining}

There is high confidence that permafrost thaw has had negative impacts on the integrity of infrastructure in high mountain areas. Like in polar regions (Section 3.4.3.3.4), the local effects of infrastructure together with climate change degraded permafrost beneath and around structures (Dall'Amico et al., 2011; Doré et al., 2016) Infrastructure on permafrost in the European Alps, mostly found near mountain summits but not in major valleys, has been destabilised by permafrost thaw, including mountain stations in France and Austria (Ravanel et al., 2013; Keuschnig et al., 2015; Duvillard et al., 2019) as well as avalanche defence structures (Phillips and Margreth, 2008) and a ski lift (Phillips et al., 2007) in Switzerland. On the Tibetan Plateau, deformation or damage has been found on roads (Yu et al., 2013; Chai et al., 2018), power transmission infrastructure (Guo et al., 2016) and around an oil pipeline (Yu et al., 2016). For infrastructure on permafrost, engineering practices suitable for polar and high mountain environments (Doré et al., 2016) as well as specific for steep terrain (Bommer et al., 2010) have been developed to support adaptation.

In some mountain regions, glacier retreat and related processes of change in the cryosphere have afforded greater accessibility for extractive industries and related activities to mine minerals and metals (medium confidence). Accelerated glacier shrinkage and retreat have been reported to facilitate mining activities in Chile, Argentina and Peru (Brenning, 2008; Brenning and Azócar, 2010; Anacona et al., 2018), and Kyrgyzstan (Kronenberg, 2013; Petrakov et al., 2016), which also interact with and have consequences for other social, cultural, economic, political and legal measures, where climate change impacts also play a role (Brenning and Azócar, 2010; Evans et al., 2016; Khadim, 2016; Anacona et al., 2018). However, negative impacts due to cryosphere changes may also occur. One study projects that reductions in glacier melt water and snowmelt in the watershed in the Chilean Andes will lead to a reduction of water supply to a copper mine by $2075-2100$ of $28 \%$ under scenario $A 2$ and of $6 \%$ under B2; construction of infrastructure to draw water from other sources will cost between 16-137 million USD (Correa-lbanez et al., 2018).

Conversely, there is also evidence suggesting that some of these mining activities affect glaciers locally, and the mountain environment around them, further altering glacier dynamics, glacier structure and permafrost degradation. This is due mainly to excavation, extraction, and use of explosives (Brenning, 2008; Brenning and Azócar, 2010; Kronenberg, 2013), and deposition of dust and other mine waste material close to or top of glaciers during extraction and transportation (Brenning, 2008; Torgoev and Omorov, 2014; Arenson et al., 2015b; Jamieson et al., 2015). These activities have reportedly generated slope instabilities (Brenning, 2008; Brenning and Azócar, 2010; Torgoev and Omorov, 2014), glacier mass loss due to enhanced surface melt from dust and debris deposition (Torgoev and Omorov, 2014; Arenson et al., 2015b; Petrakov et al., 2016), and even glacier advance by several kilometres (Jamieson et al., 2015), although their impact is considered less than that reported for changes in glaciers due to climatic change (limited evidence, medium agreement). Glacier Protection Laws and similar measures have been introduced in countries such as Chile and Argentina to address these impacts (Khadim, 2016; Anacona et al., 2018; Navarro et al., 2018). In addition, the United Nations Human Rights Council passed a declaration in 2018 to "protect and restore water-related ecosystems" in mountain areas as elsewhere from contamination by mining (UNHRC, 2018); however, evidence on the effectiveness of these measures remains inconclusive.

\subsubsection{Tourism and Recreation}

The mountain cryosphere provides important aesthetic, cultural, and recreational services to society (Xiao et al., 2015). These services support tourism, providing economic contributions and livelihood options to mountain communities and beyond. The relevant changes in the cryosphere affecting mountain tourism and recreation include shorter seasons of snow cover, more winter precipitation falling as rain instead of snow, and declining glaciers and permafrost 
(Sections 2.2.1, 2.2.2, 2.2.3 and 2.2.4). Downhill skiing, the most popular form of snow recreation, occurs in 67 countries (Vanat, 2018). The Alps in Europe support the largest ski industry (Vanat, 2018). In Europe, the growth of alpine skiing and winter tourism after 1930 brought major economic growth to alpine regions and transformed winter sports into a multi-billion USD industry (Denning, 2014). Sixteen percent of skier visits occur in the USA, where expenditures from all recreational snow sports generated more than 695,000 jobs and 72.7 billion USD in trip-related spending in 2016 (Outdoor Industry Association, 2017). While the number of ski resorts in the USA has been decreasing since the 1980s, China added 57 new ski resorts in 2017 (Vanat, 2018). Although the bulk of economic activity is held within mountain communities, supply chains for production of ski equipment and apparel span the globe. Steiger et al. (2017) point out that Asia, Africa and South America are underrepresented in the ski tourism literature, and Africa and the Middle East are not significant markets from a ski tourism perspective.

Skiing's reliance on favourable atmospheric and snow conditions make it particularly vulnerable to climate change (Arent et al., 2014; Hoegh-Guldberg etal., 2018). Snow reliability, although not universally defined, quantifies whether the snow cover is sufficient for ski resorts operations. Depending on the context, it focuses on specific periods of the winter season, and may account for interannual variability and/or for snow management (Steiger et al., 2017). The effects of less snow, due to strong correlation between snow cover and skier visits, cost the USA economy 1 billion USD and 17,400 jobs per year between 2001-2016 in years of less seasonal snow (Hagenstad et al., 2018). Efforts to reduce climate change impacts and risks to economic losses focus on increased snowmaking, such as artificial production of snow (Steiger et al., 2017), summertime slope preparation (Pintaldi et al., 2017), grooming (Steiger et al., 2017), and snow farming, that is, storage of snow (Grünewald et al., 2018). The effectiveness of snow management methods as adaptation to long-term climate change depends on sufficiently low air temperature conditions needed for snowmaking, water and energy availability, compliance with environmental regulations (de Jong, 2015), and ability to pay for investment and operating costs. When these requirements are met, evidence over the past decades shows that snow management methods have generally proven efficient in reducing the impact of reduced natural snow cover duration for many resorts (Dawson and Scott, 2013; Hopkins and Maclean, 2014; Steiger et al., 2017; Spandre et al., 2019a). The number of skier visits was found to be $39 \%$ less sensitive to natural snow variations in Swiss ski resorts with $30 \%$ areal snowmaking coverage (representing the national average), compared to resorts without snowmaking (Gonseth, 2013). In some regions, many resorts (mostly smaller, low-elevation resorts) have closed due to unfavourable snow conditions brought on by climate change and/or the associated need for large capital investments for snowmaking capacities (e.g., in northeast USA; Beaudin and Huang, 2014). To offset loss in ski tourism revenue, a key adaptation strategy is diversification, offering other non-snow recreation options such as mountain biking, mountain coasters and alpine slides, indoor climbing walls and water parks, festivals and other special events (Figure 2.9; Hagenstad et al., 2018; Da Silva et al., 2019).

In the near term (2031-2050) and regardless of the greenhouse gas emission scenario, risks to snow reliability exist for many resorts, especially at lower elevation, although snow reliability is projected to be maintained at many resorts in North America (Wobus et al., 2017) and in the European Alps, Pyrenees and Scandinavia (Marke et al., 2015; Steiger et al., 2017; Scott et al., 2019; Spandre et al., 2019a; Spandre et al., 2019b). At the end of the century (2081-2100), under RCP8.5, snow reliability is projected to be unviable for most ski resorts under current operating practices in North America, the European Alps and Pyrenees, Scandinavia and Japan, with some exceptions at high elevation or high-latitudes (Steiger et al., 2017; Wobus et al., 2017; Suzuki-Parker et al., 2018; Scott et al., 2019; Spandre et al., 2019a; Spandre et al., 2019b). Only few studies have used RCP2.6 in the context of ski tourism, and results indicate that the risks at the end of the century (2081-2100) are expected to be similar to the near term impacts (2031-2050) for RCP8.5 (Scott et al., 2019; Spandre et al., 2019a).

The projected economic losses reported in the literature include an annual loss in hotel revenues of EUR 560 million (2012 value) in Europe, compared to the period 1971-2000 under a $2^{\circ} \mathrm{C}$ global warming scenario (Damm et al., 2017). This estimate includes population projections but does not account for snow management. In the USA, Wobus et al. (2017) estimate annual revenue losses from tickets (skiing) and day fees (cross country skiing and snowmobiling) due to reduced snow season length, will range from 340-780 million USD in 2050 for RCP4.5 and RCP8.5, respectively, and from 130 million to 2 billion USD in 2090 for RCP4.5 and RCP8.5 respectively, taking into account snow management and population projections. Total economic losses from these studies would be much higher if all costs were included (costs for tickets, transport, lodging, food and equipment). Regardless of the climate scenario, as risk of financial unviability increases, there are reported expectations that companies would need to forecast when their assets may become stranded assets and require devaluation or conversion to liabilities, and report this on their balance sheets (Caldecott et al., 2016). Economic impacts are projected to occur in other snow-based winter activities including events (e.g., ski races) and other recreation activities such as cross-country skiing, snowshoeing, backcountry skiing, ice climbing, sledding, snowmobiling and snow tubing. By 2050, 13 (out of 21) prior Olympic Winter Games locations are projected to exhibit adequate snow reliability under RCP2.6, and 10 under RCP8.5. By 2080, the number decreases to 12 and 8, respectively (Scott et al., 2018). Even for cities remaining cold enough to host ski competitions, costs are projected to rise for making and stockpiling snow, as was the case in Sochi, Russia in 2014 and Vancouver, Canada in 2010 (Scott et al., 2018), and preserving race courses through salting (Hagenstad et al., 2018).

In summer, cryosphere changes are impacting glacier-related activities (hiking, sightseeing, skiing, climbing and mountaineering) (Figure 2.8). In recent years, several ski resorts operating on glaciers have ceased summer operations due to unfavourable snow conditions and excessive operating costs (e.g., Falk, 2016). Snow management and snowmaking are increasingly used on glaciers (Fischer et al., 2016). Glacier retreat has led to increased moraine instability which can compromise hiker and climber safety along established trails and common access routes, for example, in Iceland (Welling et al., 2019), though it has made some areas in the Peruvian Andes more accessible to trekkers (Vuille et al., 2018). In response, some hiking routes have 
been adjusted and ladders and fixed anchors installed (Duvillard et al., 2015; Mourey and Ravanel, 2017). As permafrost thaws, rock falls on and off glaciers are increasingly observed, threatening the safety of hikers and mountaineers, for example, in Switzerland (Temme, 2015) and New Zealand (Purdie et al., 2015). Glacier retreat and permafrost thaw have induced major changes to iconic mountaineering routes in the Mont Blanc area, European Alps with impacts on mountaineering practices, such as shifts in suitable climbing seasons, and reduced route safety (Mourey and Ravanel, 2017; Mourey et al., 2019). Cryosphere decline has also reduced opportunities for ice climbing and reduced attractions for summer trekking in the Cascade Mountains, USA (Orlove et al., 2019). In response to these impacts, tour companies have shifted to new sites, diversified to offer other activities or simply reduced their activities (Furunes and Mykletun, 2012) (Figure 2.9). Steps to improve consultation and participatory approaches to understand risk perception and design joint action between affected communities, authorities and operators, are evident, for example, in Iceland (Welling et al., 2019). In some cases, new opportunities are presented such as marketing 'climate change tourism' where visitors are attracted by 'last chance' opportunities to view a glacier; for example, in New Zealand (Stewart et al., 2016), in China (Wang et al., 2010) or through changing landscapes such as new lakes, for instance in Iceland (Pórhallsdóttir and Ólafsson, 2017), or to view the loss of a glacier, for example, in the Bolivian Andes (Kaenzig et al., 2016). The opening of a trekking route promoting this opportunity created tensions between a National Park and a local indigenous community in the Peruvian Andes over the management and allocation of revenue from the route (Rasmussen, 2019). The consequences of ongoing and future glacier retreat are projected to negatively impact trekking and mountaineering in the Himalaya (Watson and King, 2018). Reduced snow cover has also negatively impacted trekking in the Himalaya, since tourists find the mountains

(a)
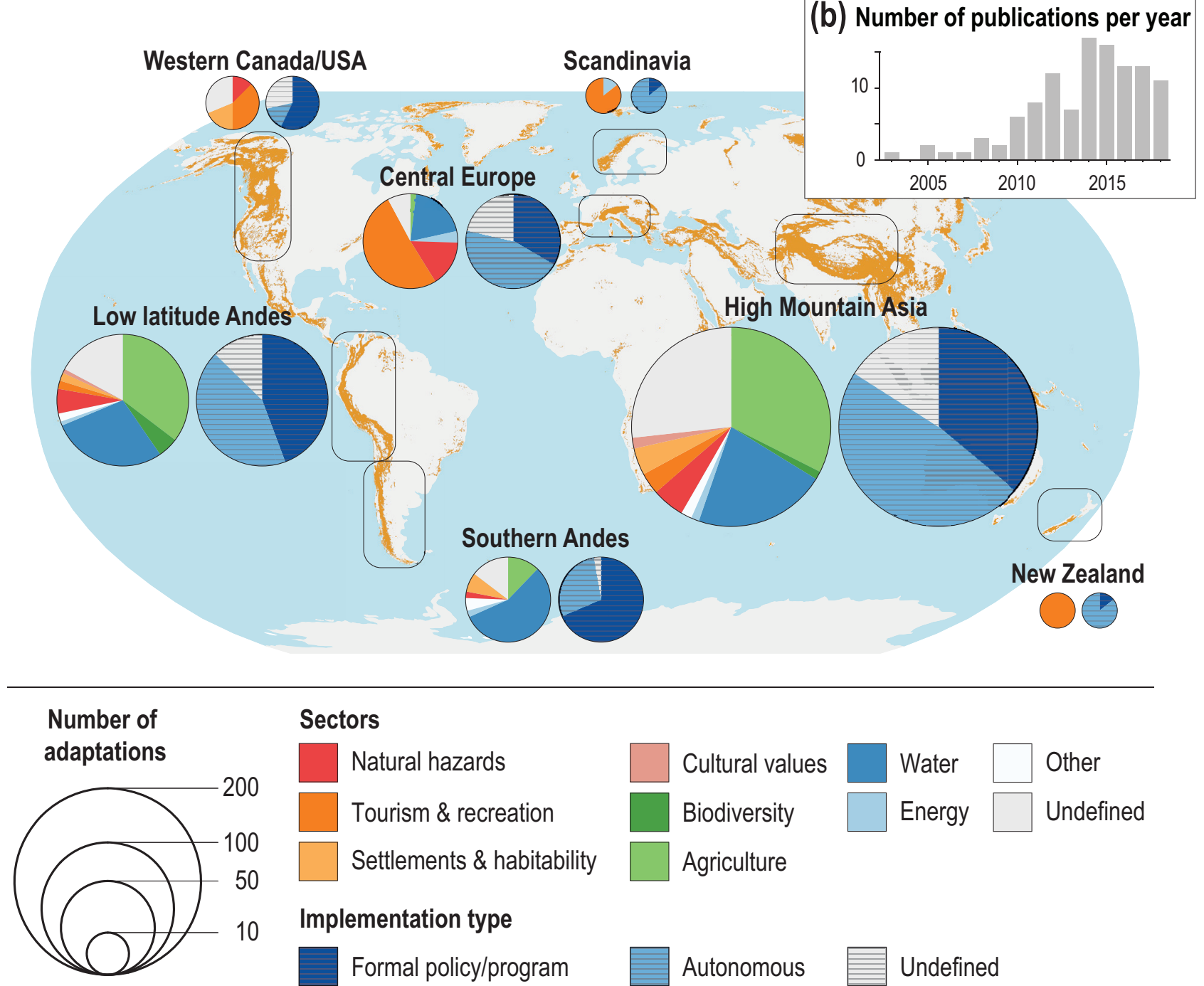

Autonomous

Undefined

Figure 2.9 | (a) Documented number of individual adaptation actions distributed across seven of the high mountain regions addressed in this Chapter, with pie charts indicating the number of adaptation measures for sectors addressed in this chapter (left pie chart), and the relative proportion of these classified as either 'formal', 'autonomous' or 'undefined' (right pie chart). Note that for regions with less than five reported adaptation measures were excluded from the figure (i.e., Caucasus, Iceland and Alaska), however these are detailed in Table SM2.9. (b) Number of publications reported in the assessed literature over time. In some cases, multiple adaptation measures are discussed in a single publication (Table SM2.9). 
less attractive as a destination, and the reduced water availability affects the ability of hotels and campsites to serve visitors (Becken et al., 2013).

In summary, financial risks to mountain communities that depend on tourism for income, are high and include losses to revenues generated from recreation primarily in the winter season. Adaptation to cryosphere change for ski tourism focuses on snowmaking and is expected to be moderately effective for many locations in the near term (2031-2050), but it is unlikely to substantially reduce the risks in most locations in the longer term (end of century) (high confidence). Determining the extent to which glacier retreat and permafrost thaw impact upon overall visitor numbers in summer tourism, and how any losses or increased costs are offset by opportunities, is inconclusive. Furthermore, tourism is also impacted by cryospheric change that impacts on water resources availability, increasing competition for its use (Section 2.3.1.3).

\subsubsection{Cultural Values and Human Well-being}

Cryosphere changes also impact cultural values, which are held by populations in high mountains and other regions around the world; these impacts often harm human well-being (Tschakert et al., 2019) (medium evidence, high agreement). Cultural values were covered extensively in AR5, with particular emphasis on small island states and the Arctic; the research on cultural values in high mountain regions is relatively new. Out of a total of 247 UNESCO World Heritage natural sites recognised for their outstanding universal value, 46 sites include glaciers within their boundaries, where the presence of glaciers is stated among the principal reason (5 sites), or secondary reason (28 sites), for World Heritage inscription; complete glacier extinction is projected by 2100 in 8 to 21 of these sites, under RCP2.6 and RCP8.5 scenarios, respectively, compromising the outstanding universal value placed on these sites, which have been inscribed at least partly for their exceptional glaciers (Bosson et al., 2019). UNESCO defines "outstanding universal value" as "cultural and/or natural significance which is so exceptional as to transcend national boundaries and to be of common importance for present and future generations of all humanity" (UNESCO, 2012). Furthermore, in recognising the importance of the cultural and intangible value placed by communities on aspects of their surrounding environment, such as those afforded by cryosphere elements in the high mountains, cultural values are mentioned under the workplan of the Warsaw International Mechanism as a specific work area under 'Non-economic loss and damage' (UNFCCC Secretariat, 2014; Serdeczny, 2019).

Cultural values include spiritual, intrinsic and existence values, as well as aesthetic dimensions, which are also an element of tourism and recreation (Section 2.3.5), though they focus more directly on ties to sacred beings or to inherent rights of entities to exist. However, these values overlap, since the visual appeal of natural landscapes links with a sense of the immensity of mountain landscapes, glaciers and fresh snow (Paden et al., 2013; Gagné et al., 2014). Moreover, different stakeholders, such as local communities, tourists and policymakers, may place different emphasis on specific cultural values (Schirpke et al., 2016). For the indigenous Manangi community of the
Annapurna Conservation Area of Nepal, the loss of glaciers which they have observed threatens their ethnic identity (Konchar et al., 2015). Villagers in the Italian Alps also report that glacier retreat weakens their identity (Jurt et al., 2015).

Spiritual and intrinsic values in high mountain regions often, but not exclusively, rest on deeply held religious beliefs and other local customs (medium evidence, high agreement). Some communities understand mountains through a religious framework (Bernbaum, 2006). In settings as diverse as the Peruvian Andes, the Nepal Himalaya, the European Alps, the North Cascades (USA), Mount Kilimanjaro and the Hengduan Mountains of southwest China, local populations view glacier retreat as the product of their failure to show respect to sacred beings or to follow proper conduct. Experiencing deep concern that they have disturbed cosmic order, they seek to behave in closer accord with established traditions; they anticipate that the retreat will continue, leading to further environmental degradation and to the decline of natural and social orders - a prospect which causes them distress (Becken et al., 2013; Gagné et al., 2014; Allison, 2015). In the USA, the snow covered peaks of the Cascades have also evoked a deep sense of awe and majesty, and an obligation to protect them (Carroll, 2012; Duntley, 2015). Similar views are found in the Italian Alps, where villagers speak of treating glacier peaks with "respect," and state that glacier retreat is due, at least in part, to humans "disturbing" the glaciers (Brugger et al., 2013), resulting in an emotion which Albrecht et al. (2007) termed solastalgia, a kind of deep environmental distress or ecological grief (Cunsolo and Ellis, 2018).

Glacier retreat threatens the Indigenous knowledge and local knowledge of populations in mountain regions; this knowledge constitutes a cultural service to wider society by contributing to scientific understanding of glaciers (Cross-Chapter Box 4 in Chapter 1). Though this knowledge is dynamic, and records previous states of glaciers, it has been undermined by the complete disappearance of glaciers in a local area (Rhoades et al., 2008). This knowledge of glaciers is often tied to religious beliefs and practices. It is based on direct observation, stories passed down from one generation to another within community, placenames, locations of structures and other sources (Gagné et al., 2014). Residents of mountain areas can provide dates for previous locations of glacier fronts, sometimes documenting these locations through the presence of structures (Brugger et al., 2013). Much like other cases of data from citizen science (Theobald et al., 2015), their observations often overlap with the record of instrumental observations (Deng et al., 2012), and can significantly extend this record (Mark et al., 2010).

An additional cultural value is the contribution of glaciers to the understanding of human history. Glacier retreat has supported the increase of knowledge of past societies by providing access to archaeological materials and other cultural resources that had previously been covered by ice. The discovery of Oetzi, a mummified Bronze Age man whose remains were discovered in 1991 in the Alps near the Italian-Austrian border, marked the beginning of scientific research with such materials (Putzer and Festi, 2014). Subsequent papers described objects that were uncovered in retreating glaciers and shrinking ice patches in the Wrangell-Saint Elias Range (Dixon 
et al., 2005), the Rocky Mountains (Lee, 2012) and Norway (Bjørgo et al., 2016). This field provides new insight into human cultural history and contributes to global awareness of climate change (Dixon et al., 2014). Though climate change permits the discovery of new artefacts and sites, it also threatens these objects and places, since they become newly exposed to harsh weather (Callanan, 2016).

\subsubsection{Migration, Habitability and Livelihoods}

High mountain communities have historically included mobility in their sets of livelihood strategies, as a means to gain access to production zones at different elevations within mountain zones and in lowland areas, and as a response to the strong seasonality of agricultural and pastoral livelihoods. Cryosphere changes in high mountain areas have influenced human mobility and migration during this century by altering water availability and increasing exposure to mass movements and floods and other cryospheric induced disasters (Figure 2.7) (Barnett et al., 2005; Carey et al., 2017; Rasul and Molden, 2019). These changes affect three forms of human mobility: transhumant pastoralism, temporary or permanent wage labour migration and displacement, in which entire communities resettle in new areas.

Transhumant pastoralism, involving movements between summer and winter pastures, is a centuries old practice in high mountain areas (Lozny, 2013). In High Mountain Asia and other regions, it is declining due to both climatic factors, including changes in snow distribution and glaciers, and to non-climatic factors, and is projected to continue declining, at least in the short term (medium evidence, high agreement). The changes in snow and glaciers adversely affect herders at their summer residences and winter camps in the Himalaya (Namgay et al., 2014) and in Scandinavian mountains (Mallory and Boyce, 2018). Reduced winter snowfall has led to poorer pasture quality in Nepal (Gentle and Maraseni, 2012) and India (Ingty, 2017). Other climate change impacts, including erratic snowfall patterns and a decrease in rainfall, are perceived by herders in Afghanistan, Nepal and Pakistan to have resulted in vegetation of lower quality and quantity (Shaoliang et al., 2012; Joshi et al., 2013; Gentle and Thwaites, 2016). Heavy snowfall incidents in winter caused deaths of a large number of livestock in northern Pakistan in 2009 (Shaoliang et al., 2012). Herders in Nepal reported of water scarcity in traditional water sources along migration routes (Gentle and Thwaites, 2016). Increased glacier melt water has caused lakes on the Tibetan Plateau to increase in size, covering pasture areas and leading pastoralists to alter their patterns of seasonal movement (Nyima and Hopping, 2019). However, rising temperatures, with associated effects on snow cover, have some positive impacts. Seasonal migration from winter to summer pastures start earlier in Northern Pakistan, and residence in summer pasture lasts longer (Joshi et al., 2013), as it does in Afghanistan (Shaoliang et al., 2012).

Wage labour migration is also a centuries old practice in the Himalaya, the Andes and the European Alps (Macfarlane, 1976; Cole, 1985; Viazzo, 1989). Studies show that migration is a second-order effect of cryosphere changes, since the first-order effects, a decrease in agricultural production (Section 2.3.1.3.2), have led to increased wage labour migration to provide supplementary income in a number of regions (medium evidence, high agreement). Wage labour migration linked to cryosphere changes occurs on several time scales, including short-term, long-term and permanent migration, and on different spatial scales. Though migration usually takes place within the country of origin, and sometimes within the region, cases of international migration have also been recorded (Merrey et al., 2018). The studies since AR5 on migration driven by cryosphere changes are concentrated in High Mountain Asia and the Andes, supporting the finding, reported in AR5 Working Group II (Section 12.7), that stress on livelihoods is an important driver of climate change induced migration. The research on such migration also supports the finding in SR15 (Section 4.3.5.6) that migration can have mixed outcomes on reducing socioeconomic vulnerability, since cases of increase and of reduction of vulnerability are both found in migration from high mountain regions that is driven by cryosphere changes.

Changing water availability, mass movements and floods are cryosphere processes which drive wage labour migration (medium evidence, high agreement). A debris flow in central Nepal in 2014, in a region where landslides have increased in recent decades, led more than half the households to migrate for months (van der Geest and Schindler, 2016). In the Santa River drainage, Peru, rural populations have declined $10 \%$ between 1970-2000, and the area of several major subsistence crops also declined (Bury et al., 2013). Research in this region suggests that seasonal wage labour migration from small basins within the main Santa basin is largest in the small drainages in which glacier retreat has reduced melt water flow most significantly; where this process is not as acute, and streamflow is less reduced, migration rates are lower (Wrathall et al., 2014). A study from a region in the central Peruvian Andes shows that the residents of the villages that have the highest dependence on glacier melt water travel further and stay away longer than the residents of the villages where glacier melt water forms a smaller portion of stream flow (Milan and $\mathrm{Ho}, 2014)$. However, the inverse relation between reliance on cryosphere-related water sources and migration was noted in a case in the Naryn River drainage in Kyrgyzstan, where the villages that are more dependent on glacier melt water had lower, rather than higher, rates of wage labour migration than the villages which were less dependent on it; the villages with lower rates of such migration also had more efficient water management institutions than the others (Hill et al., 2017). Several studies, which project cryosphere-related emigration to continue in the short term, emphasise decreased water availability, due to glacier retreat as a driver in Kyrgyzstan (Chandonnet et al., 2016) and Peru (Oliver-Smith, 2014), and to reduced snow cover in Nepal (Prasain, 2018). In most cases, climate is only one of several drivers (employment opportunities and better educational and health services in lowland areas are others).

Several studies show that wage labour migration is more frequent among young adults than among other age groups, supporting the observation in AR5 that climate change migrants worldwide are concentrated in this age (limited evidence, high agreement). This age-specific pattern is found in a valley in Northern Pakistan in which agriculture relies on glacier melt water for irrigation; as river flow decreases, the returns to agricultural labour have declined, and emigration has increased, particularly among the youth, who are 
assigned, by local cultural practices, to carry out the heaviest work (Parveen et al., 2015). Emigration has increased in recent decades from two valleys in highland Bolivia which rely on glacier melt water, as water supplies have declined, though other factors also contribute to emigration, including land fragmentation, increasing household needs for income, the lack of local wage-labour opportunities and an interest among the young in educational opportunities located in cities (Brandt et al., 2016). In Nepal, young members of high-elevation pastoral households impacted by cryosphere change have been increasingly engaged in tourism and labour migration since 2000 (Shaoliang et al., 2012); similar responses are reported for Sikkim in the Indian Himalaya (Ingty, 2017). A recent study documents the inter-generational dynamics of emigration from a livestock raising community in the Peruvian Andes, where glacier retreat has led to reduced streamflow that supports crucial dry season pasture (Alata et al., 2018). Though people 50 years old or older in this community are accustomed to living in the high pasture zones, younger people use livestock raising as a means of accumulating capital. They sell off their animals and move to towns at lower elevations. This loss of young adults has reduced the capacity of households to undertake the most demanding tasks, particularly in periods of inclement weather, accelerating the decline of herding. As a result, the human and animal populations of the communities are shrinking.

Recent research on cryosphere driven migration shows some cases of complex livelihood interactions or feedback loops, in which migration is not merely a result of changes in agricultural livelihoods, but also has impacts, either positive or negative, on these livelihoods (medium confidence). In some instances, the different livelihood strategies complement each other to support income and well-being. A review of migration in the Himalaya and Hindu Kush found that households that participated in labour migration and received remittances had improved adaptive capacity, and lowered exposure to natural hazards (Banerjee et al., 2018). In other cases, the households and communities, which undertake wage labour migration, encounter conflicts or incompatibilities between migration and agricultural livelihoods. Sustainable management of land, water and other resources is highly labour intensive, and hence labour mobility constrains and limits the adoption of sustainable practices (Gilles et al., 2013). Moreover, the labour available to a household is differentiated by age. In Northern Pakistan, where cryosphere changes are reducing streamflow the emigration of young people has led to a decline not only in the labour in fields and orchards, but also a decline in the maintenance of irrigation infrastructure, leading to an overall reduction of the agricultural livelihoods in the community (Parveen et al., 2015).

In addition to affecting pastoral transhumance and increasing wage labour migration, cryosphere changes impact human mobility by creating cases of displacement. These cases differ from wage labour migration because they involve entire communities. As a result, they are irreversible, unlike cases in which individuals undertake long-term or permanent migration from their communities but retain the possibility of returning, because, for example, some relatives or former neighbours have remained in place. In this way, these cases of displacement represent cryosphere driven challenges to habitability. Though natural hazards have historically led some communities to relocate, cryosphere changes have contributed to instances of displacement. Unreliable water availability and increased risks of natural hazards are responsible for resettlement of villages in certain high mountain areas (McDonald, 1989; Parveen et al., 2015). A village in Western Nepal moved to lower elevation after decreasing snowfall reduced the flow of water in the river on which their pastoralism and agriculture depended (Barnett et al., 2005). Three villages in Nepal faced severe declines in agricultural and pastoral livelihoods because decreased snow cover led to reduced soil moisture and to the drying up of springs, which were the historical source of irrigation water; in conjunction with an international non-governmental organisation (INGO), the residents planned a move to a lower area (Prasain, 2018).

The issue of habitability arises in the cases, mentioned above, of communities that relocate after floods or debris flows destroy houses and irrigation infrastructure, or damage fields and pastures. It occurs as well in the cases of households with extensive long-term migration, where agricultural and pastoral livelihoods are undermined by reduced water supply caused by cryospheric change (Barnett et al., 2005). In addition, the loss of cultural values, including spiritual and intrinsic values (Section 2.3.6), can contribute to decisions to migrate (Kaenzig, 2015). Combined with the patterns of permanent emigration, this issue of habitability raises the issue of limits to adaptation in mountain areas (Huggel et al., 2019). Projections of decreased streamflow by 2100 in watersheds with strong glacier melt water components in Asia, Europe, and North and South America (Section 2.3.1.1) indicate that threats to habitability may continue through this period and affect the endeavours of achieving the SDGs in developing countries (Rasul et al., 2019).

\subsection{International Policy Frameworks and Pathways to Sustainable Development}

The governance of key resources that are affected by climate-related changes in the cryosphere, such as water, is a relevant aspect for climate resilient sustainable development in mountains at the catchment level (Section 2.3.1.4). In this section, we address broader policy frameworks that are expected to shape a solution space through global action. An important development since AR5, at the global level, is the adoption of key frameworks that include the Paris Agreement (UNFCCC, 2015), UN 2030 Agenda and its SDGs (UN, 2015), and the Sendai Framework for Disaster Risk Reduction (UNISDR, 2015), which call for integrated and coordinated climate adaptation action that is also relevant for and applicable in mountain regions.

In international climate policy, the importance of averting, minimising and addressing loss and damage associated with adverse impacts of climate change is articulated in the Paris Agreement under Article 8, more specifically (UNFCCC, 2015). However, despite evident impacts of climate change on the mountain cryosphere (Section 2.3.2), there is limited evidence or reference in the literature to loss and damage for mountains, globally (Huggel et al., 2019). With already committed and unavoidable climate change, its effects on the high mountain cryosphere (Section 2.2) and related impacts and risks (Section 2.3), substantial adverse effects are expected in the coming 
decades (Huggel et al., 2019), especially at high emission scenarios, which renders this issue a relevant aspect for planning climate resilient development in mountains. At least in one region, a concrete example for responding to and translating the Paris Agreement in a transboundary mountain setting, is reported. In 2015, through policy measures afforded by the Alpine Convention for the European Alps, the ministers for the environment of the Alpine countries established the Alpine Climate Board, who at the XV Alpine Conference in April 2019, presented a climate target system that includes strategic targets for 'climate-resilient Alps' (Hojesky et al., 2019). The implementation and monitoring of these initiatives, however, remains to be assessed on an evidentiary basis. Furthermore, mechanisms afforded through the workplan of the Warsaw International Mechanism, specifically its work area under 'Non-economic loss and damage', are prospects relevant to address impacts to cultural and intrinsic values associated with losses in the high mountain cryosphere (UNFCCC Secretariat, 2014; Serdeczny, 2019).

Monitoring and reporting on progress towards sustainable development through the implementation of the SDGs (UN, 2015) is receiving some research attention in the context of mountain regions (Rasul and Tripura, 2016; Gratzer and Keeton, 2017; Bracher et al., 2018; Wymann von Dach et al., 2018; Kulonen et al., 2019; Mishra et al., 2019), noting key mountain specific considerations to improve the conditions under which the SDGs may serve a purpose in the mountain context. For example, previous research has identified a need for disaggregated data for SDG indicators and targets at subnational scales, with relevant area units that are both within country boundaries and/or across borders in transboundary settings (Rasul and Tripura, 2016; Bracher et al., 2018; Wymann von Dach et al., 2018 ). Furthermore, the use of non-standardised proxy data can further limit the potential for comparisons between countries and within regions (Bracher et al., 2018; Kulonen et al., 2019). On substance, assessments of the economic performance of livelihood options, combined with robust socioeconomic data for mountain systems, are still lacking in many parts of the world, compromising the ability for meaningful comparison and aggregation of data and knowledge for monitoring and reporting on progress of SDGs at regional or global scales (Gratzer and Keeton, 2017).

Disasters associated with natural hazards in high mountains are placing many communities and their potential for sustainable development at risk (Wymann von Dach et al., 2017; Keiler and Fuchs, 2018; Vaidya et al., 2019). The Sendai Framework for Disaster Risk Reduction 2015-2030 (UNISDR, 2015) offers a global policy framework under which risks, including climate change, can be accounted for and addressed at national scales. However, there is limited evidence in monitoring and reporting on progress on targets therein (Wymann von Dach et al., 2017), particularly in systematically reporting on root causes of disasters in high mountains and associated compounded risks and cascading impacts, and even more so when accounting for impacts related to climate change. Technical guidelines available for the high mountain context provide complementary means to monitor and report on the effectiveness of measures to reduce associated risks with changes in the cryosphere (e.g., GAPHAZ, 2017).
Other relevant frameworks include the Convention Concerning the Protection of the World Cultural and Natural Heritage, enacted to protect the planet's most significant and irreplaceable places from loss or damage (UNESCO, 1972). In it, conservation strategies are listed that aim at preserving natural and cultural heritage across regions, including sites that contain glaciers (Section 2.3.6), and are suggested as means to further support efforts towards the promotion of knowledge, collective cultural memory and climate policy (Bosson et al., 2019).

Overall, there are promising prospects through international policy frameworks to support governance and adaptation to climate-related changes in the mountain cryosphere whilst addressing sustainable development, with evidence suggesting that treaties and conventions are relevant enablers to support cooperation and implementation at the mountain region scale (Dinar et al., 2016). However, there is limited evidence to systematically assess for effectiveness in addressing specific challenges posed by changes in the mountain cryosphere, globally.

\subsection{Key Gaps in Knowledge and Prospects}

Impacts associated with climate-related changes in the high mountain cryosphere are evident in the observations reported in this chapter (Section 2.3). However, uncertainties remain with detection and attribution of key atmospheric drivers that influence much of these climate-related changes (Section 2.2.1), due to limited spatial density and/or temporal extent of observation records at high elevations. For example, trends in total or solid precipitation at high elevation remain highly uncertain, due to intrinsic uncertainties with in situ observation methods, and large natural variability. There are clear knowledge gaps in the distribution and characteristics of cryospheric variables, in particular the extent and ice content of permafrost in mountains, but also current glacier ice volumes, trends in lake and river ice, and the spatial and temporal variation of snow cover. These knowledge gaps persist despite a wealth of new data since AR5 especially from Earth observation satellites, which overcome much of the remoteness and inaccessibility of high mountains yet still face challenges for observations in mountains such as dealing with cloud cover and rugged terrain. Along with improved capacities to generate and integrate diverse observation data, initiatives such as citizen science (e.g., Dickerson-Lange et al., 2016; Wikstrom Jones et al., 2018) or Indigenous knowledge and local knowledge (Section 1.8.2, Cross Chapter Box 4 in Chapter 1) can also complement some observations that are based on conventional instruments and models. Radiative forcing effects of light absorbing particles, and understanding their spatiotemporal dynamics, is a key knowledge gap for the attribution of changes in high mountain snow and glaciers, and the understanding of regional feedbacks (Section 2.2.2, Box 2.2).

These observational knowledge gaps currently impede efforts to quantify trends, and to calibrate and evaluate models that simulate the past and future evolution of the cryosphere and its impacts. Specific uncertainties are associated with projections of future climate change trends at high elevations due mostly to current 
limits in regional climate models and downscaling methods to capture the subtle interplays between large-scale climate change and local phenomena influenced by complex topography and high relief (Section 2.2.1). Coarse-scale simulations of future permafrost conditions in high mountains are fraught with difficulties in capturing fine-scale variation of topography, surface cover and near-surface materials (Section 2.2.4). Improved cross-disciplinary studies bringing together current observation and modelling approaches in each specific field hold potential to contribute to addressing these gaps in the future.

Experiences with changes in water availability, and with changes in frequency and/or magnitude of natural hazards, demonstrate the relevance of integrated approaches to understand past impacts and prepare for future risks, where exposure and the underpinning existing vulnerabilities of mountain socioecological systems influence the extent of these impacts (Section 2.3.2.3). However, there is insufficient understanding of the effects of cryospheric change on some natural hazards such as glacier outburst floods and on infrastructure, for example for transportation. Increased wildfire risk with a shrinking cryosphere is an uncertainty both spatially and temporally and with consequent effects on mountain ecosystems, particularly with respect to soil carbon and potential biome shifts. Overall, few studies have taken a comprehensive risk approach to systematically characterise and compare magnitude and extent of past impacts and future risks across high mountain regions, including compound risks and cascading impacts where instances of deep uncertainty in responses and outcomes may arise (Cross Chapter Box 5 in Chapter 1). Furthermore, a key knowledge gap is the capacity to economically quantify cryosphere-specific impacts and potential risks.

With ecosystems, particularly the terrestrial component, uncertainty exists at which community changes can be directly linked to cryospheric change as distinct from those due to atmospheric warming. In some cases, the changes can be linked, for example, where a receding glacier creates new habitat, but rising air temperature allow some species to establish that would not otherwise be able to. A major research gap is in our understanding of the fate of legacy pollutants such as mercury downstream of their release from glaciers and permafrost in terms of quantity and regional differences, freshwater sinks, and potential effects to ecosystems and human health. Similarly, the effect of permafrost thaw on water quality and ecosystems due to the increasing release of natural heavy metals and nutrients represents a gap in knowledge.

While adaptation measures are reported for high mountain cryosphere changes (Figure 2.9 a), it stands as a relatively new and developing area of research since AR5 (Figure $2.9 \mathrm{~b}$ ), with particular gaps in terms of systematically evaluating their cost-benefits and long-term effectiveness as 'fit-for-purpose' solutions in the mountain context. Improved inter-comparability of successful adaptation cases, including the transferability of evidence for how adaptation can address both climate change and sustainable development objectives in different mountain regions, are prospects to support an evidentiary basis for future assessments of adaptation to cryosphere changes in the high mountains (Adler et al., 2019; McDowell et al., 2019).

\section{Acknowledgements}

We acknowledge the kind contributions of Matvey Debolskiy (Unversity of Alaska Fairbanks, USA), Florian Hanzer (University of Innsbruck, Austria), Andreas F. Prein (National Center for Atmospheric Research, Boulder, CO, USA), Silvia Terzago (Institute of Atmospheric

Sciences and Climate, National Research Council, Torino, Italy) and Natalia Zazulie (CONICET/University of Buenos Aires, Argentina) who contributed to drafting figures. 


\section{References}

Aas, K.S. et al., 2016: The climatic mass balance of Svalbard glaciers: a 10-year simulation with a coupled atmosphere-glacier mass balance model. The Cryosphere, 10(3), 1089-1104, doi:10.5194/tc-10-1089-2016.

Abbott, B.W. et al., 2014: Elevated dissolved organic carbon biodegradability from thawing and collapsing permafrost. J. Geophys. Res-Biogeo., 119(10), 2049-2063, doi:10.1002/2014JG002678.

Addor, N. et al., 2014: Robust changes and sources of uncertainty in the projected hydrological regimes of Swiss catchments. Water Resour. Res., 50(10), 7541-7562, doi:10.1002/2014wr015549.

Adler, C., C. Huggel, B. Orlove and A. Nolin, 2019: Climate change in the mountain cryosphere: impacts and responses. Reg. Environ. Change, 19(5), 1225-1228, doi:10.1007/s10113-019-01507-6.

Aguilera, E., M.T. Pareschi, M. Rosi and G. Zanchetta, 2004: Risk from lahars in the northern valleys of Cotopaxi volcano (Ecuador). Nat. Hazards, 33(2), 161-189, doi:10.1023/B:NHAZ.0000037037.03155.23.

Aiken, G.R. et al., 2014: Influences of glacier melt and permafrost thaw on the age of dissolved organic carbon in the Yukon River basin. Global Biogeochem. Cy., 28(5), 525-537, doi:10.1002/2013GB004764.

Åkerman, H.J. and M. Johansson, 2008: Thawing permafrost and thicker active layers in sub-arctic Sweden. Permafrost Periglac., 19(3), 279-292, doi:10.1002/ppp.626.

Alata, E., B. Fuentealba, J. Recharte, B. Fuentealba and J. Recharte, 2018: El despoblamiento de la Puna: efectos del cambio climático y otros factores. Revista Kawsaypacha, 2, 49-69, doi:10.18800/ kawsaypacha.201802.003.

Albrecht, G. et al., 2007: Solastalgia: the distress caused by environmental change. Australas. Psychiatry, 15(1), S95-S98, doi:10.1080/10398560701701288.

Aleksandrova, M., J.P.A. Lamers, C. Martius and B. Tischbein, 2014: Rural vulnerability to environmental change in the irrigated lowlands of Central Asia and options for policy-makers: A review. Environ. Sci. Policy, 41, 7788, doi:10.1016/j.envsci.2014.03.001.

Ali, S.A., S. Aadhar, H.L. Shah and V. Mishra, 2018: Projected increase in hydropower production in India under climate change. Sci. Rep., 8(1), 12450, doi:10.1038/s41598-018-30489-4.

Allen, S. and C. Huggel, 2013: Extremely warm temperatures as a potential cause of recent high mountain rockfall. Glob. Planet. Change, 107, 59-69, doi:10.1016/j.gloplacha.2013.04.007.

Allen, S.K. et al., 2018: Translating the concept of climate risk into an assessment framework to inform adaptation planning: Insights from a pilot study of flood risk in Himachal Pradesh, Northern India. Environ. Sci. Policy, 87, 1-10, doi:10.1016/j.envsci.2018.05.013.

Allen, S.K., S.C. Cox and I.F. Owens, 2011: Rock avalanches and other landslides in the central Southern Alps of New Zealand: a regional study considering possible climate change impacts. Landslides, 8(1), 33-48, doi:10.1007/s10346-010-0222-z.

Allen, S.K. et al., 2016a: Glacial lake outburst flood risk in Himachal Pradesh, India: an integrative and anticipatory approach considering current and future threats. Nat. Hazards, 84(3), 1741-1763. doi:10.1007/s11069016-2511-x.

Allen, S.K. et al., 2016b: Lake outburst and debris flow disaster at Kedarnath, June 2013: hydrometeorological triggering and topographic predisposition. Landslides, 13(6), 1479-1491, doi:10.1007/s10346-015-0584-3.

Allison, E.A., 2015: The spiritual significance of glaciers in an age of climate change. WiRes. Clim. Change, 6(5), 493-508, doi:10.1002/wcc.354.

Amagai, Y., G. Kudo and K. Sato, 2018: Changes in alpine plant communities under climate change: Dynamics of snow-meadow vegetation in northern Japan over the last 40 years. Applied Vegetation Science, 21, 561-571. doi:10.1111/avsc.12387.
Anacona, P.I. et al., 2018: Glacier protection laws: Potential conflicts in managing glacial hazards and adapting to climate change. Ambio, 47(8), 835-845, doi:10.1007/s13280-018-1043-x.

Anacona, P.I., A. Mackintosh, and K.P. Norton, K.P., 2015a: Hazardous processes and events from glacier and permafrost areas: lessons from the Chilean and Argentinean Andes. Earth Surf. Process. Landf., 40(1), 2-21, doi:10.1002/esp.3524.

Anacona, P.I., A. Mackintosh and K. Norton, 2015b: Reconstruction of a glacial lake outburst flood (GLOF) in the Engaño valley, chilean patagonia: Lessons for GLOF risk management. Sci. Total Environ., 527-528, 1-11, doi:10.1016/j.scitotenv.2015.04.096.

Ancey, C. and V. Bain, 2015: Dynamics of glide avalanches and snow gliding. Rev. Geophys., 53(3), 745-784, doi:10.1002/2015RG000491.

Andreassen, L.M. et al., 2005: Glacier mass-balance and length variation in Norway. Ann. Glaciol., 42, 317-325, doi:10.3189/172756405781812826.

Arenson, L.U., W. Colgan and H.P. Marshall, 2015a: Chapter 2 - Physical, Thermal, and Mechanical Properties of Snow, Ice, and Permafrost. In: Snow and Ice-Related Hazards, Risks, and Disasters [Shroder, J.F., W. Haeberli and C. Whiteman (eds.)]. Academic Press, Boston, pp. 35-75. ISBN 9780123948496.

Arenson, L.U., M. Jakob and P. Wainstein, 2015b: Effects of dust deposition on glacier ablation and runoff at the Pascua-Lama Mining Project, Chile and Argentina. In: Engineering Geology for Society and Territory - Volume 1: Climate Change and Engineering Geology [Lollino, G., A. Manconi, J. Clague, W. Shan and M. Chiarle (eds.)], Springer International Publishing, Cham, pp. 27-32. ISBN 9783319093000.

Arent, D.J. et al., 2014: Key economic sectors and services. In: Climate Change 2014 Impacts, Adaptation and Vulnerability: Part A: Global and Sectoral Aspects [Field, C.B., V.R. Barros, D.J. Dokken, K.J. Mach and M.D. Mastrandrea (eds.)]. Cambridge University Press, Cambridge, pp. 659-708. ISBN 9781107415379.

Armstrong, R.L. and E. Brun, 2008: Snow and climate: physical processes, surface energy exchange and modelling. Cambridge University Press, Cambridge, 256 pp. ISBN 9780521854542.

Arnold, C., T.A. Ghezzehei and A.A. Berhe, 2014: Early spring, severe frost events, and drought induce rapid carbon loss in high elevation meadows. PLOS ONE, 9(9), e106058, doi:10.1371/journal.pone.0106058.

Ashraf, A., R. Naz and R. Roohi, 2012: Glacial lake outburst flood hazards in Hindukush, Karakoram and Himalayan ranges of Pakistan: Implications and risk analysis. Geomat. Nat. Haz. Risk, 3(2), 113-132, doi:10.1080/19 475705.2011.615344.

Atmeh, K., A. Andruszkiewicz and K. Zub, 2018: Climate change is affecting mortality of weasels due to camouflage mismatch. Sci Rep, 8 (7648), doi:10.1038/s41598-018-26057-5.

Auer, I. et al., 2007: HISTALP - historical instrumental climatological surface time series of the Greater Alpine Region. Int. J. Climatol., 27 (1), 17-46, doi:10.1002/joc.1377.

Azam, M.F. et al., 2018: Review of the status and mass changes of Himalayan-Karakoram glaciers. J. Glaciol., 64(243), 61-74, doi:10.1017/ jog.2017.86.

Azócar, G.F., A. Brenning and X. Bodin, 2017: Permafrost distribution modelling in the semi-arid Chilean Andes. The Cryosphere, 11 (2), 877890, doi:10.5194/tc-11-877-2017.

Bajracharya, S.R., 2010: Glacial Lake Outburst Flood Disaster Risk Reduction Activities in Nepal. International Journal of Erosion Control Engineering, 3(1), 92-101, doi:10.13101/ijece.3.92.

Ballesteros-Cánovas, J.A. et al., 2018: Climate warming enhances snow avalanche risk in the Western Himalayas. PNAS, 115 (13), 3410-3415, doi:10.1073/pnas.1716913115. 
Bamber, J.L., R.M. Westaway, B. Marzeion and B. Wouters, 2018: The land ice contribution to sea level during the satellite era. Environ. Res. Lett., 13(6), 063008, doi:10.1088/1748-9326/aac2f0.

Banerjee, S., R. Black, A. Mishra and D. Kniveton, 2018: Assessing vulnerability of remittance-recipient and non-recipient households in rural communities affected by extreme weather events: Case studies from south-west China and northeast India. Popul. Space Place, 25(2), e2157, doi:10.1002/ psp.2157.

Baraer, M. etal., 2012: Glacier recession and water resources in Peru's Cordillera Blanca. J. Glaciol., 58(207), 134-150, doi:10.3189/2012JoG11J186.

Bard, A. et al., 2015: Trends in the hydrologic regime of Alpine rivers. J. Hydrol., 529, 1823-1837, doi:10.1016/j.jhydrol.2015.07.052.

Barnett, T.P., J.C. Adam and D.P. Lettenmaier, 2005: Potential impacts of a warming climate on water availability in snow-dominated regions. Nature, 438(7066), 303-309, doi:10.1038/nature04141.

Barr, I.D. et al., 2018: Volcanic impacts on modern glaciers: A global synthesis. Earth-Sci. Rev., 182, 186-203, doi:10.1016/j.earscirev.2018.04.008.

Barrett, K. and K. Bosak, 2018: The role of place in adapting to climate change: A case study from Ladakh, Western Himalayas. Sustainability, 10(4), 898, doi:10.3390/su10040898.

Barriga Delgado, L.M., F. Drenkhan and C. Huggel, 2018: Proyectos multipropósito para la gestión de recursos hídricos en los Andes tropicalesplanteamientos generales basados en proceso participativo [Multi-purpose Projects for Water Resources Management in the Tropical Andes: Patricipatory-based approaches]. Espacio y Desarrollo, 32, 7-28, doi:10.18800/espacioydesarrollo.201802.001.

Bashir, F., X. Zeng, H. Gupta and P. Hazenberg, 2017: A Hydrometeorological Perspective on the Karakoram Anomaly Using Unique Valley-Based Synoptic Weather Observations. Geophys. Res. Lett., 44(20), 10,41010,478, doi:10.1002/2017GL075284.

Beamer, J.P., D.F. Hill, A.A. Arendt and G.E. Liston, 2016: High-resolution modeling of coastal freshwater discharge and glacier mass balance in the Gulf of Alaska watershed. Water Resour. Res., 52(5), 3888-3909, doi:10.1002/2015WR018457.

Beaudin, L. and J.C. Huang, 2014: Weather conditions and outdoor recreation: A study of New England ski areas. Ecol. Econ., 106, 56-68, doi:10.1016/j. ecolecon.2014.07.011.

Becken, S., A.K. Lama and S. Espiner, 2013: The cultural context of climate change impacts: Perceptions among community members in the Annapurna Conservation Area, Nepal. Environ. Dev., 8, 22-37, doi:10.1016/J. ENVDEV.2013.05.007.

Bellaire, S. et al., 2016: Analysis of long-term weather, snow and avalanche data at Glacier National Park, B.C., Canada. Cold Reg. Sci. Technol., 121, 118-125, doi:10.1016/j.coldregions.2015.10.010.

Beniston, M. et al., 2018: The European mountain cryosphere: a review of its current state, trends, and future challenges. The Cryosphere, 12(2), 759794, doi:10.5194/tc-12-759-2018.

Beniston, M. and D.G. Fox, 1996: Impacts of climate change on mountain regions. In: Climate change 1995 - Impacts, adaptations and mitigation of climate change: scientific-technical analysis. Contribution of Working Group II to the Second Assessment Report of the Intergovernmental Panel of Climate Change. [Watson, R., M.C. Zinyowera and R.H. Moss (eds.)]. Cambridge University Press, Cambridge, United Kingdom and New York, NY, WGII, 191-213.

Beniston, M. and M. Stoffel, 2014: Assessing the impacts of climatic change on mountain water resources. Sci. Total Environ., 493, 1129-1137, doi:10.1016/j.scitotenv.2013.11.122.

Beniston, M. and M. Stoffel, 2016: Rain-on-snow events, floods and climate change in the Alps: Events may increase with warming up to $4^{\circ} \mathrm{C}$ and decrease thereafter. Sci. Total Environ., 571, 228-236, doi:10.1016/j. scitotenv.2016.07.146.
Benn, D.I. et al., 2012: Response of debris-covered glaciers in the Mount Everest region to recent warming, and implications for outburst flood hazards. Earth-Sci. Rev., 114(1-2), 156-174, doi:10.1016/j.earscirev.2012.03.008.

Berman, E E., N.C. Coops, S.P. Kearney and G.B. Stenhouse, 2019: Grizzly bear response to fine spatial and temporal scale spring snow cover in Western Alberta. PLOS ONE, 14(4), e0215243. doi:10.1371/journal.pone.0215243.

Bernauer, T. and T. Siegfried, 2012: Climate change and international water conflict in Central Asia. J. Peace Res., 49(1), 227-239, doi:10.1177/0022343311425843.

Bernbaum, E., 2006: Sacred mountains: Themes and teachings. Mt. Res. Dev., 26(4), 304-309, doi:10.1659/0276-4741(2006)26[304:smtat]2.0.co;2.

Berthier, E. and F. Brun, 2019: Karakoram geodetic glacier mass balances between 2008 and 2016: persistence of the anomaly and influence of a large rock avalanche on Siachen Glacier. J. Glaciol., 65(251), 494-507, doi:10.1017/jog.2019.32.

Bevington, A. and L. Copland, 2014: Characteristics of the last five surges of Lowell Glacier, Yukon, Canada, since 1948. J. Glaciol., 60(219), 113-123, doi:10.3189/2014JoG13J134.

Bhambri, R., K. Hewitt, P. Kawishwar and B. Pratap, 2017: Surge-type and surge-modified glaciers in the Karakoram. Sci. Rep., 7, 15391, doi:10.1038/ s41598-017-15473-8.

Biemans, H. et al., 2019: Importance of snow and glacier melt water for agriculture on the Indo-Gangetic Plain. Nat. Sustain. 2(7).

Bieniek, P.A. et al., 2018: Assessment of Alaska rain-on-snow events using dynamical downscaling. J. Appl. Meteorol. Climatol., 57(8), 1847-1863, doi:10.1175/JAMC-D-17-0276.1.

Bindoff, N.L. et al., 2013: Chapter 10 - Detection and attribution of climate change: From global to regional. In: Climate Change 2013: The Physical Science Basis. IPCC Working Group I Contribution to AR5 [Stocker, T.F., G.K. Plattner, M. Tignor, S.K. Allen, J. Boschung, A. Nauels, Y. Xia, V. Bex and P.M. Midgley (eds.)]. Cambridge University Press, Cambridge, United Kingdom and New York, NY, USA, Cambridge. 867-952.

Biskaborn, B.K. et al., 2019: Permafrost is warming at a global scale. Nat. Commun., 10(1), 264, doi:10.1038/s41467-018-08240-4.

Bjørgo, T. et al., 2016: Fragments of a Late Iron Age Sledge Melted Out of the Vossaskavlen Snowdrift Glacier in Western Norway. Journal of Glacial Archaeology, 2(0), 73-81, doi:10.1558/jga.v2i1.27719.

Björnsson, H., 2003: Subglacial lakes and jökulhlaups in Iceland. Glob. Planet. Change, 35(3-4), 255-271, doi:10.1016/S0921-8181(02)00130-3.

Björnsson, H. and F. Pálsson, 2008: Icelandic glaciers. Jökull, 58, 365-386.

Björnsson, H. et al., 2013: Contribution of Icelandic ice caps to sea level rise: Trends and variability since the Little Ice Age. Geophys. Res. Lett., 40(8), 1546-1550, doi:10.1002/grl.50278.

Blair, R.W., 1994: Moraine and valley wall collapse due to rapid deglaciation in Mount Cook National Park, New Zealand. Mt. Res. Dev., 14(4), 347-358, doi:10.2307/3673731.

Bliss, A., R. Hock and V. Radić, 2014: Global response of glacier runoff to twenty-first century climate change. J. Geophys. Res-Earth., 119(4), 717730, doi:10.1002/2013JF002931.

Bocchiola, D., 2014: Long term (1921-2011) hydrological regime of Alpine catchments in Northern Italy. Adv. Water Resour., 70, 51-64, doi:10.1016/j. advwatres.2014.04.017.

Bocchiola, D., M.G. Pelosi and A. Soncini, 2017: Effects of hydrological changes on cooperation in transnational catchments: the case of the Syr Darya. Water Int., 42(7), 852-873, doi:10.1080/02508060.2017.1376568.

Bockheim, J.G. and J.S. Munroe, 2014: Organic Carbon Pools and Genesis of Alpine Soils with Permafrost: A Review. Arct. Antarct. Alp. Res., 46, 9871006, doi:10.1657/1938-4246-46.4.987.

Bodin, X. et al., 2016: The 2006 Collapse of the Bérard Rock Glacier (Southern French Alps). Permafrost Periglac., 28(1), 209-223, doi:10.1002/ppp.1887.

Bodin, X., F. Rojas and A. Brenning, 2010: Status and evolution of the cryosphere in the Andes of Santiago (Chile, 33.5 ${ }^{\circ}$ S.). Geomorphology, 118, 453-464, doi:10.1016/j.geomorph.2010.02.016. 
Bodin, X. et al., 2009: Two Decades of Responses (1986-2006) to Climate by the Laurichard Rock Glacier, French Alps. Permafrost Periglac., 20(4), 331-344, doi:10.1002/ppp.665.

Boeckli, L., A. Brenning, S. Gruber and J. Noetzli, 2012: Permafrost distribution in the European Alps: calculation and evaluation of an index map and summary statistics. The Cryosphere, 6(4), 807-820, doi:10.5194/tc-6-807-2012.

Bogdal, C. et al., 2010: Release of legacy pollutants from melting glaciers: Model evidence and conceptual understanding. Environ. Sci. Technol., 44(11), 4063-4069, doi:10.1021/es903007h.

Bolch, T., T. Pieczonka, K. Mukherjee and J. Shea, 2017: Brief communication: Glaciers in the Hunza catchment (Karakoram) have been nearly in balance since the 1970s. The Cryosphere, 11 (1), 531-539, doi:10.5194/tc-11531-2017.

Bolch, T. et al., 2018: Status and Change of the Cryosphere in the Extended Hindu Kush Himalaya Region. The Hindu Kush Himalaya Assessment Mountains, Climate Change, Sustainability and People, Springer Nature, Switzerland. ISBN 9783319922874.

Bommer, C., M. Phillips and L.U. Arenson, 2010: Practical recommendations for planning, constructing and maintaining infrastructure in mountain permafrost. Permafrost Periglac., 21(1), 97-104, doi:10.1002/ppp.679.

Bonfils, C. et al., 2008: Detection and attribution of temperature changes in the mountainous Western United States. J. Clim., 21(23), 6404-6424, doi:10.1175/2008JCLI2397.1.

Bonnaventure, P.P. and A.G. Lewkowicz, 2011: Modelling climate change effects on the spatial distribution of mountain permafrost at three sites in northwest Canada. Clim. Change, 105(1-2), 293-312, doi:10.1007/ s10584-010-9818-5.

Bonnaventure, P.P., A.G. Lewkowicz, M. Kremer and M.C. Sawada, 2012: A Permafrost Probability Model for the Southern Yukon and Northern British Columbia, Canada. Permafrost Periglac., 23(1), 52-68, doi:10.1002/ ppp.1733.

Bonzanigo, L. et al., 2015: South Asia investment decision making in hydropower: decision tree case study of the Upper Arun Hydropower Project and Koshi Basin Hydropower Development in Nepal. Report No. AUS11077. World Bank, Washington, D.C., 127 pp.

Bormann, K.J., R.D. Brown, C. Derksen and T.H. Painter, 2018: Estimating snow cover trends from space. Nat. Clim. Change, 8(11), 924, doi:10.1038/ s41558-018-0318-3.

Bosshard, T., S. Kotlarski, M. Zappa and C. Schär, 2014: Hydrological climate-impact projections for the Rhine River: GCM-RCM uncertainty and separate temperature and precipitation effects. J. Hydrometeorol., 15(2), 697-713, doi:10.1175/JHM-D-12-098.1.

Bosson, J.-B., M. Huss and E. Osipova, 2019: Disappearing World Heritage Glaciers as a Keystone of Nature Conservation in a Changing Climate. Earth's Future, 7 (4), 469-479, doi:10.1029/2018ef001139.

Box, J.E. et al., 2018: Global sea-level contribution from Arctic land ice: 19712017. Environ. Res. Lett., 13(12), 125012, doi:10.1088/1748-9326/aaf2ed.

Bracher, C.P., S.W. von Dach and C. Adler, 2018: Challenges and Opportunities in Assessing Sustainable Mountain Development Using the UN Sustainable Development Goals. Universitat Bern, Bern, 42 pp.

Brahney, J. et al., 2017: Evidence for a climate-driven hydrologic regime shift in the Canadian Columbia Basin. Can. Water. Resour. J. 42(2), 179-192, do i:10.1080/07011784.2016.1268933.

Brandt, R., R. Kaenzig and S. Lachmuth, 2016: Migration as a risk management strategy in the context of climate change: Evidence from the Bolivian Andes. In: Global Migration Issues, IOMS(6) [Milan, A., B. Schraven, K. Warner and N. Cascone (eds.)]. Springer International Publishing Ag, Cham, 43-61.

Braun, M. and E. Fournier, 2016: Adaptation Case Studies in the Energy Sector - Overcoming Barriers to Adaptation, Report presented to Climate Change Impacts and Adaptation Division. Natural Resources Canada, pp. 114. ISBN 9782923292229.
Braun, M.H. et al., 2019: Constraining glacier elevation and mass changes in South America. Nat. Clim. Change, 9(2), 130-136, doi:10.1038/s41558018-0375-7.

Brenning, A., 2008: The impact of mining on rock glaciers and glaciers. In: Darkening peaks: glacier retreat, science, and society [Orlove, B.S., E. Weigandt and B. Luckman (eds.)]. University of California Press, Berkely, 196-205.

Brenning, A. and G.F. Azócar, 2010: Minería y glaciares rocosos: impactos ambientales, antecedentes políticos y legales, y perspectivas futuras. Revista de geografía Norte Grande, 47, 143-158, doi:10.4067/S071834022010000300008.

Brinkerhoff, D., M. Truffer and A. Aschwanden, 2017: Sediment transport drives tidewater glacier periodicity. Nat. Commun., 8(1), 90, doi:10.1038/ s41467-017-00095-5.

Brown, L.E., D.M. Hannah and A.M. Milner, 2007: Vulnerability of alpine stream biodiversity to shrinking glaciers and snowpacks. Glob. Change Biol, 13(5), 958-966, doi:10.1111/j.1365-2486.2007.01341.x.

Brown, L.E. et al., 2018: Functional diversity and community assembly of river invertebrates show globally consistent responses to decreasing glacier cover. Nat. Ecol. Evol., 2(2), 325-333, doi:10.1038/s41559-017-0426-x.

Brugger, J., K.W. Dunbar, C. Jurt and B. Orlove, 2013: Climates of anxiety: Comparing experience of glacier retreat across three mountain regions. Emote. Space Soc., 6, 4-13, doi:10.1016/j.emospa.2012.05.001.

Brun, F. et al., 2017: A spatially resolved estimate of High Mountain Asia glacier mass balances, 2000-2016. Nat. Geosci., 10(9), 668-673, doi:10.1038/NGE02999.

Brunner, M.I. et al., 2019: Present and future water scarcity in Switzerland: Potential for alleviation through reservoirs and lakes. Sci. Total Environ., 666, 1033-1047, doi:10.1016/j.scitotenv.2019.02.169.

Buchli, T. et al., 2013: Characterization and monitoring of the furggwanghorn rock glacier, Turtmann Valley, Switzerland: Results from 2010 to 2012. Vadose Zone J., 12, doi:10.2136/vzj2012.0067.

Buckel, J., J.C. Otto, G. Prasicek and M. Keuschnig, 2018: Glacial lakes in Austria - Distribution and formation since the Little Ice Age. Glob. Planet. Change, 164, 39-51, doi:10.1016/j.gloplacha.2018.03.003.

Büntgen, U. et al., 2017: Elevational range shifts in four mountain ungulate species from the Swiss Alps. Ecosphere, 8(4), e01761, doi:10.1002/ecs2.1761.

Burns, P. and A. Nolin, 2014: Using atmospherically-corrected Landsat imagery to measure glacier area change in the Cordillera Blanca, Peru from 1987 to 2010. Remote Sens. Environ., 140, 165-178, doi:10.1016/j.rse.2013.08.026.

Bury, J. et al., 2013: New geographies of water and climate change in Peru: Coupled natural and social transformations in the Santa River Watershed. Ann. Am. Assoc. Geogr., 103 (2), 363-374, doi:10.1080/00045608.2013. 754665.

Bury, J.T. et al., 2011: Glacier recession and human vulnerability in the Yanamarey watershed of the Cordillera Blanca, Peru. Clim. Change, 105 (1-2), 179-206, doi:10.1007/s10584-010-9870-1.

Buytaert, W. and B. De Bièvre, 2012: Water for cities: The impact of climate change and demographic growth in the tropical Andes. Water Resour. Res., 48 (8), 897, doi:10.1029/2011WR011755.

Buytaert, W. et al., 2017: Glacial melt content of water use in the tropical Andes. Environ. Res. Lett., 12, 1-8, doi:10.1088/1748-9326/aa926c.

Cai, Y. et al., 2019: Variations of lake ice phenology on the Tibetan Plateau From 2001 to 2017 based on MODIS Data. J. Geophys. Res. Atmos., 124(2), 825-843. doi:10.1029/2018jd028993.

Caldecott, B. et al., 2016: Stranded Assets: A Climate Risk Challenge [Rios, A.R. (ed.)]. Inter-American Development Bank. [Available at: https://publications.iadb.org/handle/11319/7946]. Accessed 05/08/2019.

Callanan, M., 2016: Managing frozen heritage: Some challenges and responses. Quaternary Int., 402, 72-79, doi:10.1016/j.quaint.2015.10.067.

Camac, J.S. et al., 2017: Climatic warming strengthens a positive feedback between alpine shrubs and fire. Global Change Biol., 23(8), 3249-s3258, doi:10.1111/gcb.13614. 
Cao, B. et al., 2018: Thermal characteristics and recent changes of permafrost in the upper reaches of the Heihe River Basin, Western China. J. Geophys. Res-Atmos., 123(15), 7935-7949, doi:10.1029/2018JD028442.

Capell, R., D. Tetzlaff, R. Essery and C. Soulsby, 2014: Projecting climate change impacts on stream flow regimes with tracer-aided runoff modelspreliminary assessment of heterogeneity at the mesoscale. Hydrol. Process. 28(3), 545-558, doi:10.1002/hyp.9612.

Carey, M., 2005: Living and dying with glaciers: people's historical vulnerability to avalanches and outburst floods in Peru. Glob. Planet. Change, 47(2-4), 122-134, doi:10.1016/j.gloplacha.2004.10.007.

Carey, M. et al., 2014: Toward hydro-social modeling: Merging human variables and the social sciences with climate-glacier runoff models (Santa River, Peru). J. Hydrol., 518, 60-70, doi:10.1016/j.jhydrol.2013.11.006.

Carey, M., A. French and E. O'Brien, 2012: Unintended effects of technology on climate change adaptation: An historical analysis of water conflicts below Andean Glaciers. J. Hist. Geogr., 38 (2), 181-191, doi:10.1016/j. jhg.2011.12.002.

Carey, M. et al., 2017: Impacts of glacier recession and declining melt water on mountain societies. Ann Am. Assoc. Geogr., 107(2), 350-359, doi:10.10 80/24694452.2016.1243039.

Carrivick, J.L. et al., 2013: Outburst flood evolution at Russell Glacier, western Greenland: effects of a bedrock channel cascade with intermediary lakes. Quaternary Sci. Rev., 67, 39-58, doi:10.1016/j.quascirev.2013.01.023.

Carrivick, J.L. and F.S. Tweed, 2016: A global assessment of the societal impacts of glacier outburst floods. Glob. Planet. Change, 144, 1-16, doi:10.1016/j. gloplacha.2016.07.001

Carroll, B.E., 2012:Worlds in space:American religious pluralism in geographic perspective. JAAR, 80(2), 304-364, doi:10.1093/jaarel/lfs024.

Castebrunet, H. et al., 2014: Projected changes of snow conditions and avalanche activity in a warming climate: the French Alps over the 2020-2050 and 2070-2100 periods. The Cryosphere, 8(5), 1673-1697, doi:10.5194/tc-8-1673-2014.

Cauvy-Fraunié, S. et al., 2016: Ecological responses to experimental glacier-runoff reduction in alpine rivers. Nat. Commun., 7, 12025, doi:10.1038/ncomms12025.

Ceppi, P., S.C. Scherrer, A.M. Fischer and C. Appenzeller, 2012: Revisiting Swiss temperature trends 1959-2008. Int. J. Climatol., 32 (2), 203-213, doi:10.1002/joc.2260.

Chadburn, S.E. et al., 2017: An observation-based constraint on permafrost loss as a function of global warming. Nat. Clim. Change, 7(5), 340-344, doi:10.1038/nclimate3262.

Chai, M. et al., 2018: Characteristics of asphalt pavement damage in degrading permafrost regions: Case study of the Qinghai-Tibet Highway, China. J. Cold. Reg. Eng., 32(2), 05018003, doi:10.1061/(asce)cr.19435495.0000165 .

Chandonnet, A., Z. Mamadalieva and L. Orolbaeva, 2016: Environment, climate change and migration In the Kyrgyz Republic. IOM, Kyrgyzstan, 112 pp.

Chen, Y. et al., 2016: Changes in Central Asia's water tower: Past, present and future. Sci. Rep., 6, 35458, doi:10.1038/srep35458.

Chen, Y. et al., 2014: Comparison of the sensitivity of surface downward longwave radiation to changes in water vapor at two high elevation sites. Environ. Res. Lett., 9(11), 114015, doi:10.1088/1748-9326/9/11/114015.

Chevallier, P., B. Pouyaud, W. Suarez and T. Condom, 2011: Climate change threats to environment in the tropical Andes: Glaciers and water resources. Reg. Environ. Change, 11 (Suppl.1), 179-187, doi:10.1007/s10113010-0177-6.

Christiansen, H.H. et al., 2010: The thermal state of permafrost in the nordic area during the international polar year 2007-2009. Permafrost Periglac., 21(2), 156-181, doi:10.1002/ppp.687.

Chudley, T.R., E.S. Miles and I.C. Willis, 2017: Glacier characteristics and retreat between 1991 and 2014 in the Ladakh Range, Jammu and Kashmir. Remote Sens. Lett., 8(6), 518-527, doi:10.1080/2150704X.2017.1295480.
Clarke, G.K.C. et al., 2015: Projected deglaciation of western Canada in the twenty-first century. Nat. Geosci, , 8(5), 372-377, doi:10.1038/ngeo2407.

Clouse, C., 2016: Frozen landscapes: climate-adaptive design interventions in Ladakh and Zanskar. Landscape Research, 41(8), 821-837, doi:10.1080/0 1426397.2016.1172559.

Clouse, C., N. Anderson and T. Shippling, 2017: Ladakh's artificial glaciers: climate-adaptive design for water scarcity. Clim. Dev., 9(5), 428-438, doi: 10.1080/17565529.2016.1167664.

Cloutier, C. et al., 2016: Potential impacts of climate change on landslides occurrence in Canada. In: Slope Safety Preparedness for Impact of Climate Change [Ho, K., S. Lacasse and L. Picarelli (eds.)]. Taylor \& Francis Group, Florida, pp. 71-104. ISBN 978113803230.

Coe, J.A., E.K. Bessette-Kirton and M. Geertsema, 2017: Increasing rock-avalanche size and mobility in Glacier Bay National Park and Preserve, Alaska detected from 1984 to 2016 Landsat imagery. Landslides, 15(3), 393-407, doi:10.1007/s10346-017-0879-7.

Cohen, J., H. Ye and J. Jones, 2015: Trends and variability in rain-on-snow events. Geophys. Res. Lett., 42(17), 7115-7122, doi:10.1002/2015GL065320.

Cole, J.A., 1985: The Potosi mita, 1573-1700: Compulsory Indian labor in the Andes. Stanford University Press, Stanford. 206 pp.

Colombo, N. et al., 2018: Review: Impacts of permafrost degradation on inorganic chemistry of surface fresh water. Glob. Planet. Change, 162, 69-83, doi:10.1016/j.gloplacha.2017.11.017.

Colonia, D. et al., 2017: Compiling an inventory of glacier-bed overdeepenings and potential new lakes in de-glaciating areas of the peruvian andes: Approach, first results, and perspectives for adaptation to climate Change. Water, 9(5), 336, doi:10.3390/w9050336.

Comola, F., B. Schaefli, A. Rinaldo and M. Lehning, 2015: Thermodynamics in the hydrologic response: Travel time formulation and application to Alpine catchments. Water Resour. Res., 51(3), 1671-1687, doi:10.1002/2014WR016228.

Cook, N. and D. Butz, 2013: The Atta Abad Landslide and Everyday Mobility in Gojal, Northern Pakistan. Mt. Res. Dev., 33(4), 372-380, doi:10.1659/ mrd-journal-d-13-00013.1.

Coppola, E. et al., 2014: Changing hydrological conditions in the Po basin under global warming. Sci. Total Environ., 493, 1183-1196, doi:10.1016/j. scitotenv.2014.03.003.

Correa-Ibanez, R., G. Keir and N. McIntyre, 2018: Climate-resilient water supply for a mine in the Chilean Andes. In: Proceedings of the Institution of Civil Engineers - Water Management, 171(4), 203-215. doi.org/10.1680/ jwama.16.00129.

Cotto, 0. et al., 2017: A dynamic eco-evolutionary model predicts slow response of alpine plants to climate warming. Nat. Commun., 8, 15399, doi:10.1038/ncomms15399.

Cramer, W. et al., 2014: Detection and attribution of observed impacts. In: Climate Change 2014: Impacts, Adaptation, and Vulnerability. Part A: Global and Sectoral Aspects. Contribution of Working Group II to the FifthAssessment Report of the Intergovernmental Panel on Climate Change [Field, C.B., V.R. Barros, D.J. Dokken, K.J. Mach, M.D. Mastrandrea, T.E. Bilir, M. Chatterjee, K.L. Ebi, Y.O. Estrada, R.C. Genova, B. Girma, E.S. Kissel, A.N. Levy, S. MacCracken, P.R. Mastrandrea and L.L. White (eds.)]. Cambridge University Press, Cambridge, United Kingdom and New York, NY, USA, 79-1037.

Cuellar, A.D. and D.C. McKinney, 2017: Decision-making methodology for risk management applied to Imja Lake in Nepal. Water, 9(8), 591, doi:10.3390/ w9080591.

Cullen, N.J., Sirguey, P., Mölg, T., Kaser, G., Winkler, M., and Fitzsimmons, S.J., 2013: A century of ice retreat on Kilimanjaro: the mapping reloaded. The Cryosphere, 7, 419-431, doi:10.5194/tc-7-419-2013.

Cunsolo, A. and N.R. Ellis, 2018: Ecological grief as a mental health response to climate change-related loss. Nat. Clim. Change, 8(4), 275-281, doi:10.1038/s41558-018-0092-2. 
Curry, A.M., V. Cleasby and P. Zukowskyj, 2006: Paraglacial response of steep, sediment-mantled slopes to post-'Little Ice Age'glacier recession in the central Swiss Alps. J. Quat. Sci. , 21(3), 211-225, doi:10.1002/jqs.954.

Cutter, S. et al., 2012: Managing the risks from climate extremes at the local level. [Field, C.B., V. Barros, T.F. Stocker and Q. Dahe (eds.)]. Cambridge University Press, Cambridge, 291-338.

Cutter, S.L. and D.P. Morath, 2013: The evolution of the social vulnerability index (SoVI). In: Measuring Vulnerability to Natural Hazards. Towards Disaster Resilience Societies [Birkmann, J. (ed.)]. United Nations University Press, New York/Bonn, pp. 304-321.

D'Amico, M.E., M. Freppaz, E. Zanini and E. Bonifacio, 2017: Primary vegetation succession and the serpentine syndrome: the proglacial area of the Verra Grande glacier, North-Western Italian Alps. Plant Soil, 415(1-2), 283--298, doi:10.1007/s11104-016-3165-x.

Da Silva, L. et al., 2019: Analyse économique des mesures d'adaptation aux changements climatiques appliquée au secteur du ski alpin au Québec. Ouranos, Montréal, $119 \mathrm{pp}$.

Dall'Amico, M. et al., 2011: Chapter 4: Local ground movements and effects on infrastructures. In: Hazards related to permafrost and to permafrost degradation, PermaNET Project Report 6.2, pp.107-147.

Dame, J. and J.S. Mankelow, 2010:Stongde revisited: Land-use change in central Zangskar. Erdkunde, 64(4), 355-370, doi:10.3112/erdkunde.2010.04.05.

Dame, J. and M. Nüsser, 2011: Food security in high mountain regions: Agricultural production and the impact of food subsidies in Ladakh, Northern India. Food Security, 3(2), 179-194, doi:10.1007/s12571-0110127-2.

Damm, A., W. Greuell, O. Landgren and F. Prettenthaler, 2017: Impacts of $+2^{\circ} \mathrm{C}$ global warming on winter tourism demand in Europe. Climate Services, 7, 31-46, doi:10.1016/j.cliser.2016.07.003.

Dangi, M.B. et al., 2018: Impacts of environmental change on agroecosystems and livelihoods in Annapurna Conservation Area, Nepal. Environmental Development, 25, 59-72, doi:10.1016/j.envdev.2017.10.001.

Darcy, J.L. et al., 2018: Phosphorus, not nitrogen, limits plants and microbial primary producers following glacial retreat. Sci. Adv., 4(5), doi:10.1126/ sciadv.aaq0942.

Darrow, M.M. et al., 2016: Frozen debris lobe morphology and movement: An overview of eight dynamic features, southern Brooks Range, Alaska. The Cryosphere, 10(3), 977-993, doi:10.5194/tc-10-977-2016.

Dawson, J. and D. Scott, 2013: Managing for climate change in the alpine ski sector. Tourism Management, 35, 244-254, doi:10.1016/j. tourman.2012.07.009.

De Boeck, H.J. et al., 2018: Legacy effects of climate extremes in alpine grassland. Front. Plant Sci, 9(1586), doi:10.3389/fpls.2018.01586.

de Jong, C., 2015: Challenges for mountain hydrology in the third millennium. Front. Environ. Sci., 3, 38, doi:10.3389/fenvs.2015.00038.

de Kok, R.J., O.A. Tuinenburg, P.N.J. Bonekamp and W.W. Immerzeel, 2018: Irrigation as a Potential Driver for Anomalous Glacier Behavior in High Mountain Asia. Geophys. Res. Lett., 5(2), 1071-2054, doi:10.1002/2017GL076158.

De Stefano, L. et al., 2010: Mapping the Resilience of International River Basins to Future Climate Change-Induced Water Variability, Volume 1. Main Report. World Bank Water Sector Board Discussion Paper No. 15, 56051, The World Bank, Washington, DC., 88 pp.

Deems, J.S. et al., 2013: Combined impacts of current and future dust deposition and regional warming on Colourado River Basin snow dynamics and hydrology. Hydrol. Earth Syst. Sc., 17(11), 4401-4413, doi:10.5194/ hess-17-4401-2013.

Dehecq, A. et al., 2019: Twenty-first century glacier slowdown driven by mass loss in High Mountain Asia. Nat. Geosci., 12, 22-27, doi:10.1038/s41561018-0271-9.
Delaloye, R., C. Lambiel and I. Gärtner-Roer, 2010: Overview of rock glacier kinematics research in the Swiss Alps: Seasonal rhythm, interannual variations and trends over several decades. Geogr. Helv., 65, 135-145, doi:10.5194/gh-65-135-2010.

Deline, P., 2009: Interactions between rock avalanches and glaciers in the Mont Blanc massif during the late Holocene. Quaternary Sci. Rev., 28 (1112), 1070-1083, doi:10.1016/j.quascirev.2008.09.025.

Deline, P. et al., 2015: Chapter 15: Ice loss and slope stability in High-Mountain Regions. In: Snow and Ice-Related Hazards, Risks, and Disasters [Shroder, J.F., W. Haeberli and C. Whiteman (eds.)]. Elsevier, Amsterdam, 521-561.

Deng, M.Z., D.H. Qin and H.G. Zhang, 2012: Public perceptions of climate and cryosphere change in typical arid inland river areas of China: Facts, impacts and selections of adaptation measures. Quatern. Int., 282, 48-57, doi:10.1016/j.quaint.2012.04.033.

Denning, A., 2014: From Sublime Landscapes to "White Gold": How Skiing Transformed the Alps after 1930. Environ. Hist.,, 19( 1), 78-108, doi:10.1093/envhis/emt105.

Dharmadhikary, S., 2008: Mountains of Concrete: Dam Building in the Himalayas. eSocialSciences, Working Papers id:1815, [Available at: https:// ideas.repec.org/p/ess/wpaper/id1815.html]. Accessed 05/08/2019.

Di Mauro, B. et al., 2015: Mineral dust impact on snow radiative properties in the European Alps combining ground, UAV, and satellite observations. J. Geophy. Res., 120(12), 6080-6097, doi:10.1002/2015JD023287.

Díaz, S. et al., 2019: Summary for policymakers of the global assessment report on biodiversity and ecosystem services of the Intergovernmental Science-Policy Platform on Biodiversity and Ecosystem Services (advance unedited version). [Available at: www.ipbes.net/sites/default/files/ downloads/spm_unedited_advance_for_posting_htn.pdf].

Dickerson-Lange, S. et al., 2016: Challenges and successes in engaging citizen scientists to observe snow cover: from public engagement to an educational collaboration. JCOM, 15(1), A01, doi:10.22323/2.15010201.

Dileepkumar, R., K. AchutaRao and T. Arulalan, 2018: Human influence on sub-regional surface air temperature change over India. Sci. Rep., 8, 8967, doi:10.1038/s41598-018-27185-8.

Dinar, S., D. Katz, L. De Stefano and B. Blankespoor, 2016: Climate change and water variability: do water treaties contribute to river basin resilience? A review. Policy Research Working Paper 7855, The World Bank, Washington, D.C. Ding, J. et al., 2016: The permafrost carbon inventory on the Tibetan Plateau: a new evaluation using deep sediment cores. Glob. Change Biol, 22, 2688-2701, doi:10.1111/gcb.13257.

Dixon, E.J., M.E. Callanan, A. Hafner and P.G. Hare, 2014: The emergence of glacial archaeology. Journal of Glacial Archaeology, 1(1), 1-9, doi:10.1558/ jga.v1i1.1.

Dixon, E.J., W.F. Manley and C.M. Lee, 2005: The Emerging archaeology of glaciers and ice patches: Examples from Alaska's Wrangell-St. Elias National Park and Preserve. Am. Antiq., 70(1), 129-143, doi:10.2307/40035272.

Dobrowski, S.Z. and S.A. Parks, 2016: Climate change velocity underestimates climate change exposure in mountainous regions. Nat. Commun., 7, 1-8, doi:10.1038/ncomms12349.

Dolezal, J. et al., 2016: Vegetation dynamics at the upper elevational limit of vascular plants in Himalaya. Sci. Rep., 6, 1-13, doi:10.1038/srep24881.

Doré, G., F. Niu and H. Brooks, 2016: Adaptation methods for transportation infrastructure built on degrading permafrost. In: Permafrost Periglac., 27, 352-364, doi:10.1002/ppp.1919.

Drenkhan, F. et al., 2015: The changing water cycle: climatic and socioeconomic drivers of water-related changes in the Andes of Peru. WiRes.Water, 2(6), 715-733, doi:10.1002/wat2.1105.

Drenkhan, F., C. Huggel, L. Guardamino and W. Haeberli, 2019: Managing risks and future options from new lakes in the deglaciating Andes of Peru: The example of the Vilcanota-Urubamba basin. Sci. Total Environ., 665, 465-483, doi:10.1016/j.scitotenv.2019.02.070. 
Du, J. et al., 2017: Satellite microwave assessment of Northern Hemisphere lake ice phenology from 2002 to 2015. The Cryosphere, 11(1), 47-63, doi:10.5194/tc-11-47-2017.

Duethmann, D. et al., 2015: Attribution of streamflow trends in snow and glacier melt-dominated catchments of the Tarim River, Central Asia. Water Resour. Res., 51(6), 4727-4750, doi:10.1002/2014wr016716.

Duguay, C.R., M. Bernier, Y. Gauthier and A. Kouraev, 2014: Remote sensing of lake and river ice. In: Remote Sensing of the Cryosphere [Tedesco, M. (ed.)]. John Wiley \& Sons, Ltd, Chichester, UK, 273-306.

Dunse, T. et al., 2015: Glacier-surge mechanisms promoted by a hydro-thermodynamic feedback to summer melt. The Cryosphere, 9(1), 197-215, doi:10.5194/tc-9-197-2015.

Duntley, M., 2015: Spiritual Tourism and Frontier Esotericism at Mount Shasta, California. International Journal for the Study of New Religions, 5(2), 123-150, doi:10.1558/ijsnr.v5i2.26233.

Duvillard, P.A., L. Ravanel and P. Deline, 2015: Risk assessment of infrastructure destabilisation due to global warming in the high French Alps. Revue de Géographie Alpine, 103 (2), doi:10.4000/rga.2896.

Duvillard, P.A., L. Ravanel, M. Marcer and P. Schoeneich, 2019: Recent evolution of damage to infrastructure on permafrost in the French Alps. Reg. Environ. Change, 19(5), 1281-1293, doi:10.1007/s10113-019-01465-z.

Dymov, A.A., E.V. Zhangurov and F. Hagedorn, 2015: Soil organic matter composition along altitudinal gradients in permafrost affected soils of the Subpolar Ural Mountains. Catena, 131, 140-148, doi:10.1016/j. catena.2015.03.020.

Eby, L.A., O. Helmy, L.M. Holsinger and M.K. Young, 2014: Evidence of climate-induced range contractions in bull trout Salvelinus confluentus in a Rocky Mountain watershed, USA. PLOS ONE, 9(6), doi:10.1371/journal. pone.0098812.

Eckert, N. et al., 2013: Temporal trends in avalanche activity in the French Alps and subregions: from occurrences and runout altitudes to unsteady return periods. J. Glaciol., 59(213), 93-114, doi:10.3189/2013JoG12J091.

Eichel, J., D. Draebing and N. Meyer, 2018: From active to stable: Paraglacial transition of Alpine lateral moraine slopes. Land Degrad. Dev., 29(11), 4158-4172, doi:10.1002/ldr.3140.

Einarsson, B. and S. Jónsson, 2010: The effect of climate change on runoff from two watersheds in Iceland. Icelandic Meteorological Office, Reykjavik. $34 \mathrm{pp}$.

Eisen, O., W.D. Harrison and C.F. Raymond, 2001: The surges of variegated glacier, Alaska, U.S.A., and their connection to climate and mass balance. J. Glaciol., 47(158), 351-358, doi:10.3189/172756501781832179.

Elsen, P.R., W.B. Monahan and A.M.Merenlender, 2018: Global patterns of protection of elevational gradients in mountain ranges. Proc. Natl. Acad. Sci. U.S.A., 115(23), 6004-6009, doi:10.1073/pnas.1720141115.

Elsen, P.R. and M.W. Tingley, 2015: Global mountain topography and the fate of montane species under climate change. Nat. Clim. Change, 5(8), 772-776, doi:10.1038/nclimate2656.

Emmer, A. et al., 2016: 882 lakes of the Cordillera Blanca: An inventory, classification, evolution and assessment of susceptibility to outburst floods. Catena, 147, 269-279, doi:10.1016/j.catena.2016.07.032.

Engelhardt, M. et al., 2017: Melt water runoff in a changing climate (19512099) at Chhota Shigri Glacier, Western Himalaya, Northern India. Ann. Glaciol., 58(75), 47-58, doi:10.1017/aog.2017.13.

Engeset, R.V., T.V. Schuler and M. Jackson, 2005: Analysis of the first jökulhlaup at Blåmannsisen, northern Norway, and implications for future events. Ann. Glaciol. , 42, 35-41, doi:10.3189/172756405781812600.

Eriksen, H. et al., 2018: Recent Acceleration of a Rock Glacier Complex, Ádjet, Norway, Documented by 62 Years of Remote Sensing Observations. Geophys. Res. Lett., 45(16), 8314-8323, doi:10.1029/2018GL077605.

Erokhin, S.A. et al., 2017: Debris flows triggered from non-stationary glacier lake outbursts: the case of the Teztor Lake complex (Northern Tian Shan, Kyrgyzstan). Landslides, 15(1), 83-98, doi:10.1007/s10346-017-0862-3.
Evangelista, A. et al., 2016: Changes in composition, ecology and structure of high-mountain vegetation: A re-visitation study over 42 years. AoB Plants, 8, 1-11, doi:10.1093/aobpla/plw004.

Evans, D.J.A., M. Ewertowski, S.S.R. Jamieson and C. Orton, 2016: Surficial geology and geomorphology of the Kumtor Gold Mine, Kyrgyzstan: human impacts on mountain glacier landsystems. J. Maps, 12(5), 757-769. doi:10. 1080/17445647.2015.1071720.

Evans, S.G. and K.B. Delaney, 2015: Chapter 16: Catastrophic mass flows in the mountain glacial environment. In: Snow, and Ice-Related Hazards, Risks, and Disasters [Haeberli, W. and C. Whitemann (eds.)]. Elsevier, Amsterdam, 563-606.

Evans, S.G. et al., 2009: Catastrophic detachment and high-velocity long-runout flow of Kolka Glacier, Caucasus Mountains, Russia in 2002. Geomorphology, 105, 314-321, doi:10.1016/j.geomorph.2008.10.008.

Evette, A., L. Peyras, H. Francois and S. Gaucherand, 2011: Environmental risks and impacts of mountain reservoirs for artificial snow production in a context of climate change. Journal of Alpine Research | Revue de géographie alpine, (99-4), doi:10.4000/rga.1471.

Fadrique, B. et al., 2018: Widespread but heterogeneous responses of Andean forests to climate change. Nature, 564 (7735), 207-212, doi:10.1038/ s41586-018-0715-9.

Faillettaz, J., M. Funk and C. Vincent, 2015: Avalanching glacier instabilities: Review on processes and early warning perspectives. Rev. Geophys., 53(2), 203-224, doi:10.1002/2014rg000466.

Fairman, T.A., L.T. Bennett, S. Tupper and C.R. Nitschke, 2017: Frequent wildfires erode tree persistence and alter stand structure and initial composition of a fire-tolerant sub-alpine forest. J. Veg. Sci., 28(6), 11511165, doi:10.1111/jvs.12575.

Falk, M., 2016: The stagnation of summer glacier skiing. Tourism Analysis, 21(1), 117-122, doi:10.3727/108354216X14537459509053.

Farbrot, H., K. Isaksen, B. Etzelmüller and K. Gisnås, 2013: Ground thermal regime and permafrost distribution under a changing climate in northern Norway. Permafrost Periglac., 24(1), 20-38, doi:10.1002/ppp.1763.

Farinotti, D. et al., 2019: A consensus estimate for the ice thickness distribution of all glaciers on Earth. Nat. Geosci., 12, 168-173, doi:10.1038/s41561019-0300-3.

Fell, S.C., J.L. Carrivick and L.E. Brown, 2017: The multitrophic effects of climate change and glacier retreat in mountain rivers. Bioscience, 67(10), 897-911, doi:10.1093/biosci/bix107.

Fell, S.C. et al., 2018: Declining glacier cover threatens the biodiversity of alpine river diatom assemblages. Glob. Change Biol., 24(12), 5828-5840, doi:10.1111/gcb.14454.

Fellman, J.B. et al., 2015: Evidence for the assimilation of ancient glacier organic carbon in a proglacial stream food web. Limnol, Oceanogr., 60(4), 1118-1128, doi:10.1002/Ino.10088.

Fellman, J.B. et al., 2014: Watershed Glacier Coverage Influences Dissolved Organic Matter Biogeochemistry in Coastal Watersheds of Southeast Alaska. Ecosystems, 17(6), 1014-1025, doi:10.1007/s10021-014-9777-1.

Fickert, T. and F. Grüninger, 2018: High-speed colonization of bare ground-permanent plot studies on primary succession of plants in recently deglaciated glacier forelands. Land Degrad. Dev., 29(8), 2668-2680, doi:10.1002/ldr.3063.

Fickert, T., F. Grüninger and B. Damm, 2016: Klebelsberg revisited: did primary succession of plants in glacier forelands a century ago differ from today? Alpine Botany, 127(1), 17-29, doi:10.1007/s00035-016-0179-1.

Fiddes, J. and S. Gruber, 2012: TopoSUB: a tool for efficient large area numerical modelling in complex topography at sub-grid scales. Geosci. Model. Dev. 5(5), 1245-1257, doi:10.5194/gmd-5-1245-2012.

Figueroa-Armijos, M. and C.B. Valdivia, 2017: Sustainable innovation to cope with climate change and market variability in the Bolivian Highlands. Innovation and Development, 7(1), 17-35, doi:10.1080/2157 930X.2017.1281210. 
Finn, D.S., A.C. Encalada and H. Hampel, 2016: Genetic isolation among mountains but not between stream types in a tropical high-altitude mayfly. Freshw. Biol., 61(5), 702-714, doi:10.1111/fwb.12740.

Finn, D.S., K. Khamis and A.M. Milner, 2013: Loss of small glaciers will diminish beta diversity in Pyrenean streams at two levels of biological organization. Global Ecol. Biogeogr., 22(1), 40-51, doi:10.1111/j.14668238.2012.00766.x.

Fischer, A., K. Helfricht and M. Stocker-Waldhuber, 2016: Local reduction of decadal glacier thickness loss through mass balance management in ski resorts. The Cryosphere, 10(6), 2941-2952, doi:10.5194/tc-10-2941-2016.

Fischer, L., C. Huggel, A. Kääb and W. Haeberli, 2013: Slope failures and erosion rates on a glacierized high-mountain face under climatic changes. Earth Surf. Process. Landf., 38(8), 836-846, doi:10.1002/Esp.3355.

Fischer, L. et al., 2012: On the influence of topographic, geological and cryospheric factors on rock avalanches and rockfalls in high-mountain areas. Nat. Hazard. Earth Sys., 12(1), 241-254, doi:10.5194/nhess-12-241-2012.

Fischlin, A. et al., 2007: Ecosystems, their properties, goods and services. In: Climate change 2007: Impacts, adaptation and vulnerability. Contribution of Working Group II to the Fourth Assessment Report of the Intergovernmental Panel of Climate Change (IPCC) [Parry, M.L., O.F. Canziani, J.P. Palutikof, P.J. van der Linden and C.E. Hanson (eds.)]. Cambridge University Press, Cambridge, UK, 211-272.

Fleming, S.W. and H.E. Dahlke, 2014: Modulation of linear and nonlinear hydroclimatic dynamics by mountain glaciers in Canada and Norway: Results from information-theoretic polynomial selection. Can. Water. Resour. J., 39(3), 324-341, doi:10.1080/07011784.2014.942164.

Foresta, L. et al., 2016: Surface elevation change and mass balance of Icelandic ice caps derived from swath mode CryoSat-2 altimetry. Geophys. Res. Lett., 43(23), 12138-12145, doi:10.1002/2016GL071485.

Foresta, L. et al., 2018: Heterogeneous and rapid ice loss over the Patagonian Ice Fields revealed by CryoSat-2 swath radar altimetry. Remote Sens. Environ., 211, 441-455, doi:10.1016/j.rse.2018.03.041.

Forsythe, N. et al., 2017: Karakoram temperature and glacial melt driven by regional atmospheric circulation variability. Nat. Clim. Change, 7 (9), 664670, doi:10.1038/nclimate3361.

Frans, C. et al., 2015: Predicting glacio-hydrologic change in the headwaters of the Zongo River, Cordillera Real, Bolivia. Water Resour. Res., 51(11), 9029-9052, doi:10.1002/2014WR016728.

Fraser, B. 2017. Learning from flood-alarm system's fate, EcoAmericas. www.ecoamericas.com/en/story. aspx?id=1776. Accessed on 05/08/2019.

Freeman, B.G., J.A. Lee-Yaw, J.M. Sunday and A.L. Hargreaves, 2018: Expanding, shifting and shrinking: The impact of global warming on species' elevational distributions. Global Ecol. Biogeogr., 27, 1268-1276, doi:10.1111/geb.12774.

Frei, E.R. et al., 2018: Biotic and abiotic drivers of tree seedling recruitment across an alpine treeline ecotone. Sci. Rep., 8(1), doi:10.1038/s41598-01828808-w.

Freudiger, D., I. Kohn, K. Stahl and M. Weiler, 2014: Large-scale analysis of changing frequencies of rain-on-snow events with flood-generation potential. Hydrol. Earth Syst. Sc., 18(7), 2695-2709, doi:10.5194/hess-182695-2014.

Frey, H. et al., 2010: A multi-level strategy for anticipating future glacier lake formation and associated hazard potentials. Nat. Hazard. Earth Sys., 10(2), 339-352, doi:10.5194/nhess-10-339-2010.

Fuchs, M., P. Kuhry and G. Hugelius, 2015: Low below-ground organic carbon storage in a subarctic Alpine permafrost environment. The Cryosphere, 9(2), 427-438, doi:10.5194/tc-9-427-2015.

Fuhrer, J., P. Smith and A. Gobiet, 2014: Implications of climate change scenarios for agriculture in alpine regions--a case study in the Swiss Rhone catchment. Sci. Total Environ., 493, 1232-1241, doi:10.1016/j. scitotenv.2013.06.038.

Fujita, K. et al., 2013: Potential flood volume of Himalayan glacial lakes. Nat. Hazard. Earth Sys., 13(7), 1827-1839, doi:10.5194/nhess-13-1827-2013.
Fürst, J.J. et al., 2018: The ice-free topography of Svalbard. Geophys. Res. Lett., 45 (21), 11,760-11,769, doi:10.1029/2018GL079734.

Furunes, T. and R.J. Mykletun, 2012: Frozen adventure at risk? A 7-year follow-up study of Norwegian glacier tourism. Scandinavian Journal of Hospitality and Tourism, 12(4), 324-348, doi:10.1080/15022250.2012.748507.

Gadek, B. et al., 2017: Snow avalanche activity in Żleb Żandarmerii in a time of climate change (Tatra Mts., Poland). Catena, 158, 201-212, doi:10.1016/j. catena.2017.07.005.

Gagné, K., M.B. Rasmussen and B. Orlove, 2014: Glaciers and society: Attributions, perceptions, and valuations. WiRes. Clim. Change, 5(6), 793808, doi:10.1002/wcc.315.

Gao, J., 2019: Global population projection grids based on Shared Socioeconomic Pathways (SSPs), downscaled 1-km grids, 2010-2100. Palisades, NY. doi.org/10.7927/H44747X4.

Gao, Y. et al., 2018: Does elevation-dependent warming hold true above $5000 \mathrm{~m}$ elevation? Lessons from the Tibetan Plateau. npj Climate and Atmospheric Science, 1(19). doi:10.1038/s41612-018-0030-z.

GAPHAZ, 2017: Assessment of Glacier and Permafrost Hazards in Mountain Regions - Technical Guidance Document. [Allen, S., H. Frey, C. Huggel and e. al. (eds.)]. Standing Group on Glacier and Permafrost Hazards in Mountains (GAPHAZ) of the International Association of Cryospheric Sciences (IACS) and the International Permafrost Association (IPA). Zurich, Switzerland / Lima, Peru, 72 pp.

García-Hernández, C. et al., 2017: Reforestation and land use change as drivers for a decrease of avalanche damage in mid-latitude mountains (NW Spain). Glob. Planet. Change, 153, 35-50, doi:10.1016/j.gloplacha.2017.05.001.

Gardelle, J., Y. Arnaud and E. Berthier, 2011: Contrasted evolution of glacial lakes along the Hindu Kush Himalaya mountain range between 1990 and 2009. Glob. Planet. Change, 75 (1-2), 47-55, doi:10.1016/j. gloplacha.2010.10.003.

Gardelle, J., E. Berthier and Y. Arnaud, 2012: Slight mass gain of Karakoram glaciers in the early twenty-first century. Nat. Geosci., 5(5), 322-325, doi:10.1038/ngeo1450.

Gardelle, J., E. Berthier, Y. Arnaud and A. Kääb, 2013: Region-wide glacier mass balances over the Pamir-Karakoram-Himalaya during 1999-2011. The Cryosphere, 7(4), 1263-1286, doi:10.5194/tc-7-1263-2013.

Gardner, A.S. et al., 2013: A reconciled estimate of glacier contributions to sea level rise: 2003 to 2009. Science, 340(6134), 852-857, doi:10.1126/ science. 1234532.

Gardner, J., J. Sinclair, F. Berkes and R.B. Singh, 2002: Accelerated tourism development and its impacts in Kullu-Manali, H.P., India. Tourism Recreation Research, 27(3), 9-20, doi:10.1080/02508281.2002.11081370.

Gardner, J.S. and J. Dekens, 2006: Mountain hazards and the resilience of social-ecological systems: lessons learned in India and Canada. Nat. Hazards, 41(2), 317-336, doi:10.1007/s11069-006-9038-5.

Gaudard, L., J. Gabbi, A. Bauder and F. Romerio, 2016: Long-term uncertainty of hydropower revenue due to climate change and electricity prices. Water Resour. Manage., 30(4), 1325-1343, doi:10.1007/s11269-015-1216-3.

Gaudard, L., M. Gilli and F. Romerio, 2013: Climate change impacts on hydropower management. Water Resour. Manage., 27(15), 5143-5156, doi:10.1007/s11269-013-0458-1.

Gaudard, L. et al., 2014: Climate change impacts on hydropower in the Swiss and Italian Alps. Sci. Total Environ., 493, 1211-1221, doi:10.1016/j. scitotenv.2013.10.012.

Gebre, S., T. Boissy and K. Alfredsen, 2014: Sensitivity of lake ice regimes to climate change in the Nordic region. The Cryosphere, 8 (4), 1589-1605, doi:10.5194/tc-8-1589-2014.

Geertsema, M. and J.J. Clague, 2005: Jokulhlaups at Tulsequah Glacier, northwestern British columbia, Canada. The Holocene, 15(2), 310-316, doi:10.1191/0959683605hl812rr.

Geertsema, M., J.J. Clague, J.W. Schwab and S.G. Evans, 2006: An overview of recent large catastrophic landslides in northern British Columbia, Canada. Eng. Geol., 83(1-3), 120-143, doi:10.1016/j.enggeo.2005.06.028. 
Gentili, R. et al., 2015: Potential warm-stage microrefugia for alpine plants: Feedback between geomorphological and biological processes. Ecol. Complex., 21, 87-99, doi:10.1016/j.ecocom.2014.11.006.

Gentle, P. and T.N. Maraseni, 2012: Climate change, poverty and livelihoods: adaptation practices by rural mountain communities in Nepal. Environ. Sci. Policy, 21, 24-34, doi:10.1016/j.envsci.2012.03.007.

Gentle, P. and R. Thwaites, 2016: Transhumant pastoralism in the context of socioeconomic and climate change in the mountains of Nepal. Mt. Res. Dev., 36(2), 173-182, doi:10.1659/mrd-journal-d-15-00011.1.

Giacona, F. et al., 2018: Avalanche activity and socio-environmental changes leave strong footprints in forested landscapes: a case study in the Vosges medium-high mountain range. Ann. Glaciol., 10(77), 1-23, doi:10.1017/ aog.2018.26.

Giersch, J.J. et al., 2017: Climate-induced glacier and snow loss imperils alpine stream insects. Glob. Change Biol, 23(7), 2577-2589, doi:10.1111/ gcb.13565.

Giersch, J.J. et al., 2015: Climate-induced range contraction of a rare alpine aquatic invertebrate. Freshw. Sci., 34(1), 53-65, doi:10.1086/679490.

Giesen, R.H. and J. Oerlemans, 2013: Climate-model induced differences in the 21st century global and regional glacier contributions to sea-level rise. Clim. Dyn., 41(11-12), 3283-3300, doi:10.1007/s00382-013-1743-7.

Gilbert, A. et al., 2018: Mechanisms leading to the 2016 giant twin glacier collapses, Aru Range, Tibet. The Cryosphere, 12(9), 2883-2900, doi:10.5194/tc-12-2883-2018.

Gilbert, A. et al., 2015: Assessment of thermal change in cold avalanching glaciers in relation to climate warming. Geophys. Res. Lett., 42(15), 63826390, doi:10.1002/2015GL064838.

Gilbert, A. et al., 2012: The influence of snow cover thickness on the thermal regime of Tête Rousse Glacier (Mont Blanc range, 3200 $\mathrm{m}$ a.s.l.): Consequences for outburst flood hazards and glacier response to climate change. J. Geophys. Res-Earth., 117(F4), F04018, doi:10.1029/2011JF002258.

Gilles, J.L., J.L. Thomas, C. Valdivia and E.S. Yucra, 2013: Laggards or Leaders: Conservers of Traditional Agricultural Knowledge in Bolivia. Rural Sociol., 78(1), 51-74, doi:10.1111/ruso.12001.

Giménez-Benavides, L. et al., 2018: How does climate change affect regeneration of Mediterranean high-mountain plants? An integration and synthesis of current knowledge. Plant Biology, 20, 50-62, doi:10.1111/ plb.12643.

Ginot, P. et al., 2014: A 10 year record of black carbon and dust from a Mera Peak ice core (Nepal): variability and potential impact on melting of Himalayan glaciers. The Cryosphere, 8(4), 1479-1496, doi:10.5194/tc8-1479-2014.

Gitay, H., S. Brown, W. Easterling and B. Jallow, 2001: Ecosystems and their goods and services. In: Climate Change 2001 - Impacts, Adaptation, and Vulnerability. Contribution of Working Group II to the Third Assessment Report of the Intergovernmental Panel of Climate Change (IPCC) [McCarthy, J.J., O.F. Canziani, N.A. Leary, D.J. Dokken and K.S. White (eds.)]. Cambridge University Press, Cambridge, UK, 237-342.

Gonseth, C., 2013: Impact of snow variability on the Swiss winter tourism sector: Implications in an era of climate change. Clim. Change, 119(2), 307-320, doi:10.1007/s10584-013-0718-3.

Gosai, M.A. and L. Sulewski, 2014: Urban attraction: Bhutanese internal rural-urban migration. Asian Geographer, 31(1), 1-16, doi:10.1080/102 25706.2013.790830.

Gou, P. et al., 2017: Lake ice phenology of Nam Co, Central Tibetan Plateau, China, derived from multiple MODIS data products. J. Great. Lakes Res., 43(6), 989-998, doi:10.1016/j.jglr.2017.08.011.

Goulden, M.L. and R.C. Bales, 2014: Mountain runoff vulnerability to increased evapotranspiration with vegetation expansion. PNAS, 111(39), 14071-14075, doi:10.1073/pnas.1319316111.
Graae, B.J. et al., 2018: Stay or go - how topographic complexity influences alpine plant population and community responses to climate change. Perspect. Plant. Ecol., 30, 41-50, doi:10.1016/J.PPEES.2017.09.008.

Gratzer, G. and W.S. Keeton, 2017: Mountain Forests and Sustainable Development: The Potential for Achieving the United Nations' 2030 Agenda. Mt. Res. Dev., 37(3), 246-253, doi:10.1659/MRD-JOURNAL-D-17-00093.1.

Grau, H.R. and T.M. Aide, 2007: Are rural-urban migration and sustainable development compatible in mountain systems? Mt. Res. Dev., 27(2), 119124, doi:10.1659/mrd.0906.

Groll, M. et al., 2015: Water quality, potential conflicts and solutions-an upstream-downstream analysis of the transnational Zarafshan River (Tajikistan, Uzbekistan). Environ. Earth Sci., 73(2), 743-763, doi:10.1007/ s12665-013-2988-5.

Gruber, S., 2012: Derivation and analysis of a high-resolution estimate of global permafrost zonation. The Cryosphere, 6(1), 221-233, doi:10.5194/ tc-6-221-2012.

Gruber, S. et al., 2017: Review article: Inferring permafrost and permafrost thaw in the mountains of the Hindu Kush Himalaya region. The Cryosphere, 11(1), 81-99, doi:10.5194/tc-11-81-2017.

Gruber, S. and W. Haeberli, 2007: Permafrost in steep bedrock slopes and its temperature-related destabilization following climate change. J. Geophys. Res-Oceans, 112(F2), F02S18, doi:10.1029/2006JF000547.

Grünewald, T., F. Wolfsperger and M. Lehning, 2018: Snow farming: conserving snow over the summer season. The Cryosphere, 12(1), 385400, doi:10.5194/tc-12-385-2018.

Grytnes, J.-A. et al., 2014: Identifying the driving factors behind observed elevational range shifts on European mountains. Global Ecol. Biogeogr., 23(8), 876-884, doi:10.1111/geb.12170.

Guo, D. and H. Wang, 2016: CMIP5 permafrost degradation projection: A comparison among different regions. J. Geophys. Res-Atmos., 121(9), 4499-4517, doi:10.1002/2015jd024108.

Guo, D., H. Wang and D. Li, 2012: A projection of permafrost degradation on the Tibetan Plateau during the 21st century. J. Geophys. Res-Atmos., 117, D05106, doi:10.1029/2011JD016545.

Guo, L. et al., 2016: Displacements of tower foundations in permafrost regions along the Qinghai-Tibet Power Transmission Line. Cold Reg. Sci. Technol., 121, 187-195, doi:10.1016/j.coldregions.2015.07.012.

Gupta, V. and M.P. Sah, 2008: Impact of the Trans-Himalayan Landslide Lake Outburst Flood (LLOF) in the Satluj catchment, Himachal Pradesh, India. Nat. Hazards, 45(3), 379-390, doi:10.1007/s11069-007-9174-6.

Gutmann, E. et al., 2016: The Intermediate Complexity Atmospheric Research Model (ICAR). J. Hydrometeorol., 17(3), 957-973, doi:10.1175/ jhm-d-15-0155.1.

Haeberli, W. et al., 2016: New lakes in deglaciating high-mountain regions opportunities and risks. Clim. Change, 139(2), 201-214, doi:10.1007/ s10584-016-1771-5.

Haeberli, W., A. Kääb, D.V. Mühll and P. Teysseire, 2001: Prevention of outburst floods from periglacial lakes at Grubengletscher, Valais, Swiss Alps. J. Glaciol., 47 (156), 111-122-122, doi:10.3189/172756501781832575.

Haeberli, W., Y. Schaub and C. Huggel, 2017: Increasing risks related to landslides from degrading permafrost into new lakes in de-glaciating mountain ranges. Geomorphology, 293, 405-417, doi:10.1016/j. geomorph.2016.02.009.

Haeberli, W. and C. Whiteman, 2015: Snow and Ice-related Hazards, Risks, and Disasters. Elsevier, Amsterdam. 812 pp. ISBN 9780123948496.

Hagenstad, M., E. Burakowski and R. Hill, 2018: The economic contributions of winter sports in a changing climate. Protect our winters. Hagenstad Consulting, Inc., Boulder, USA. 80 pp.

Hall, L.E., A.D. Chalfoun, E.A. Beever and A.E. Loosen, 2016: Microrefuges and the occurrence of thermal specialists: implications for wildlife persistence amidst changing temperatures. Climate Change Responses, 3(1), 8, doi:10.1186/s40665-016-0021-4. 
Hamududu, B. and A. Killingtveit, 2012: Assessing climate change impacts on global hydropower. Energies, 5(2), 305-322, doi:10.3390/en5020305.

Hänggi, P. and R. Weingartner, 2012: Variations in discharge volumes for hydropower generation in Switzerland. Water Resour. Manage., 26(5), 1231-1252, doi:10.1007/s11269-011-9956-1.

Hannah, L. et al., 2014: Fine-grain modeling of species' response to climate change: Holdouts, stepping-stones, and microrefugia. Trends Ecol. Evol., 29, 390-397, doi:10.1016/j.tree.2014.04.006.

Hardy, S.P., D.R. Hardy and K.C. Gil, 2018: Avian nesting and roosting on glaciers at high elevation, Cordillera Vilcanota, Peru. Wilson J. Ornithol., 130(4), 940--957, doi:10.1676/1559-4491.130.4.940.

Hari, R.E. et al., 2006: Consequences of climatic change for water temperature and brown trout populations in Alpine rivers and streams. Glob. Change Biol, 12(1), 10-26, doi:10.1111/j.1365-2486.2005.001051.x.

Harpold, A.A. and P.D. Brooks, 2018: Humidity determines snowpack ablation under a warming climate. PNAS, 115(6), 1215-1220, doi:10.1073/ pnas.1716789115.

Harpold, A.A. and N.P. Molotch, 2015: Sensitivity of soil water availability to changing snowmelt timing in the western U.S. Geophys. Res. Lett., 42(19), 8011-8020, doi:10.1002/2015GL065855.

Harris, C. et al., 2009: Permafrost and climate in Europe: Monitoring and modelling thermal, geomorphological and geotechnical responses. Earth-Sci. Rev., 92(3-4), 117-171, doi:10.1016/j.earscirev.2008.12.002.

Harris, C. et al., 2003: Warming permafrost in European mountains. Glob. Planet. Change, 39(3-4), 215-225, doi:10.1016/j.gloplacha.2003.04.001.

Harrison, S. et al., 2018: Climate change and the global pattern of moraine-dammed glacial lake outburst floods. The Cryosphere, 12(4), 1195-1209, doi:10.5194/tc-12-1195-2018.

Harrison, W.D. et al., 2015: Glacier Surges. In: Snow, and Ice-Related Hazards, Risks, and Disasters [Haeberli, W. and C. Whitemann (eds.)]. Elsevier, Amsterdam, 437-485.

Hartl, L., A. Fischer, M. Stocker-Waldhuber and J. Abermann, 2016: Recent speed-up of an alpine rock glacier: An updated chronology of the kinematics of outer hochebenkar rock glacier based on geodetic measurements. Geografiska Annaler. Series A, Physical Geography, 98(2), 129-141, doi:10.1111/geoa.12127.

Hartmann, D.L. et al., 2013: Observations: Atmosphere and surface. In: Climate Change 2013: The Physical Science Basis. Contribution of Working Group I to the Fifth Assessment Report of the Intergovernmental Panel on Climate Change [Stocker, T.F., D. Qin, G.-K. Plattner, M. Tignor, S.K. Allen, J. Boschung, A. Nauels, Y. Xia, V. Bex and P.M. Midgley (eds.)]. Cambridge University Press, Cambridge, United Kingdom and New York, NY, USA, 159-254.

Hartmann, H. and L. Andresky, 2013: Flooding in the Indus River basin A spatiotemporal analysis of precipitation records. Glob. Planet. Change, 107, 25-35, doi:10.1016/j.gloplacha.2013.04.002.

Hasler, A., S. Gruber, M. Font and A. Dubois, 2011: Advective heat transport in frozen rock clefts: Conceptual model, laboratory experiments and numerical simulation. Permafrost Periglac., 22(4), 378-389, doi:10.1002/ppp.737.

Hauer, C. et al., 2018: State of the art, shortcomings and future challenges for a sustainable sediment management in hydropower: A review. Renew. Sust. Energ. Rev., 98, 40-55, doi:10.1016/j.rser.2018.08.031.

Hawkings, J. et al., 2016: The Greenland Ice Sheet as a hot spot of phosphorus weathering and export in the Arctic. Global Biogeochem. Cy., 30(2), 191210, doi:10.1002/2015GB005237.

He, X., K.S. Burgess, L.M. Gao and D.Z. Li, 2019: Distributional responses to climate change for alpine species of Cyananthus and Primula endemic to the Himalaya-Hengduan Mountains. Plant Diversity, 41, 26-32, doi:10.1016/j.pld.2019.01.004.

Hegglin, E. and C. Huggel, 2008: An integrated assessment of vulnerability to glacial hazards. A case study in the Cordillera Blanca, Peru. Mt. Res. Dev., 28(3-4), 299-309, doi:10.1659/mrd.0976.
Heikkinen, A., 2017: Climate change in the Peruvian Andes: A case study on small-scale farmers' Vulnerability in the Quillcay River Basin. Iberoamericana - Nordic Journal of Latin American and Caribbean Studies, 46(1), 77-88, doi:10.16993/iberoamericana.211.

Held, I.M. and B.J. Soden, 2006: Robust responses of the hydrological cycle to global warming. J. Clim., 19(21), 5686-5699, doi:10.1175/JCLI3990.1.

Hermanns, R.L., T. Oppikofer, N.J. Roberts and G. Sandoy, 2014: Catalogue of historical displacement waves and landslide-triggered tsunamis in Norway. In Engineering Geology for Society and Territory, Vol 4: Marine and Coastal Processes [Lollino, G., A. Manconi, J. Locat, Y. Huang, and M. Canals Artigas (eds.)]. pp 63-66, doi:10.1007/978-3-319-08660-6_13.

Hewitt, K., 2007: Tributary glacier surges: an exceptional concentration at Panmah Glacier, Karakoram Himalaya. J. Glaciol., 53(181), 181-188, doi:10.3189/172756507782202829.

Higman, B. et al., 2018: The 2015 landslide and tsunami in Taan Fiord, Alaska. Sci. Rep., 8, 12993, doi:10.1038/s41598-018-30475-w.

Hilbich, C. et al., 2008: Monitoring mountain permafrost evolution using electrical resistivity tomography: A 7-year study of seasonal, annual, and long-term variations at Schilthorn, Swiss Alps. J. Geophys. Res-Earth., 113(F1), 1-12, doi:10.1029/2007JF000799.

Hill, A., C. Minbaeva, A. Wilson and R. Satylkanov, 2017: Hydrologic controls and water vulnerabilities in the Naryn River Basin, Kyrgyzstan: A socio-hydro case study of water stressors in Central Asia. Water, 9(5), 325, doi:10.3390/w9050325.

Hill, M., 2013: Adaptive capacity of water governance: Cases from the Alps and the Andes. Mt. Res. Dev., 33(3), 248-259, doi:10.1659/ MRD-JOURNAL-D-12-00106.1.

Hipp, T. et al., 2012: Modelling borehole temperatures in Southern Norway-insights into permafrost dynamics during the 20th and 21st century. The Cryosphere, 6(3), 553-571, doi:10.5194/tc-6-553-2012.

Hirabayashi, Y. et al., 2016: Contributions of natural and anthropogenic radiative forcing to mass loss of Northern Hemisphere mountain glacie

Mrs and quantifying their uncertainties. Sci. Rep., 6, 29723, doi:10.1038/ srep29723.

Hirabayashi, Y. et al., 2013: Projection of glacier mass changes under a high-emission climate scenario using the global glacier model HYOGA2. Hydrol. Res. Lett., 7(1), 6-11, doi:10.3178/hrl.7.6.

Hock, R. et al., 2019: GlacierMIP - A model intercomparison of global-scale glacier mass-balance models and projections. J. Glaciol., 65(251), 453-467, doi:10.1017/jog.2019.22.

Hodson, A.J., 2014: Understanding the dynamics of black carbon and associated contaminants in glacial systems. WiRes. Water, 1(2), 141-149, doi:10.1002/wat2.1016.

Hoegh-Guldberg, O. et al., 2018: Impacts of $1.5^{\circ} \mathrm{C}$ global warming on natural and human systems. In: Global Warming of $1.5^{\circ} \mathrm{C}$. An IPCC Special Report on the impacts of global warming of $1.5^{\circ} \mathrm{C}$ above pre-industrial levels and related global greenhouse gas emission pathways, in the context of strengthening the global response to the threat of climate change, sustainable development, and efforts to eradicate poverty [Masson-Delmotte, V., P. Zhai, H.-O. Pörtner, D. Roberts, J. Skea, P.R. Shukla, A. Pirani, W. Moufouma-Okia, C. Péan, R. Pidcock, S. Connors, J.B.R. Matthews, Y. Chen, X. Zhou, M.I. Gomis, E. Lonnoy, T. Maycock, M. Tignor and T. Waterfield (eds.)]. In Press.

Hojesky, H. et al., 2019: Alpine Climate Target System 2050 - approved by the XV Alpine Conference. Alpine Climate Board of the Alpine Convention, Innssbruck. www.alpconv.org/en/organization/groups/AlpineClimateBoard/ Documents/20190404_ACB_AlpineClimateTargetSystem2050_en.pdf. Accessed on 06/08/2019.

Hood, E. et al., 2015: Storage and release of organic carbon from glaciers and ice sheets. Nat. Geosci., 8(2), 91-96, doi:10.1038/ngeo2331.

Hood, E. et al., 2009: Glaciers as a source of ancient and labile organic matter to the marine environment. Nature, 462(7276), 1044-U100, doi:10.1038/ nature 08580 . 
Hopkins, D. and K. Maclean, 2014: Climate change perceptions and responses in Scotland's ski industry. Tourism Geographies, 16, 400-414, doi:10.1080 /14616688.2013.823457.

Hotaling, S. et al., 2018: Demographic modelling reveals a history of divergence with gene flow for a glacially tied stonefly in a changing post-Pleistocene landscape. J. Biogeogr., 45(2), 304-317, doi:10.1111/jbi.13125.

Hubbard, S.S. et al., 2018: The East River, Colorado, Watershed: A mountainous community testbed for improving predictive understanding of multiscale hydrological-biogeochemical dynamics. Vadose Zone Journal, 17(1), 180061, doi:10.2136/vzj2018.03.0061.

Huggel, C., M. Carey, J.J. Clague and A. Kääb (eds.), 2015a: The high-mountain cryosphere: Environmental changes and human risks. Cambridge University Press, Cambridge. 363 pp. ISBN 9781107065840.

Huggel, C. et al., 2019: Loss and Damage in the mountain cryosphere. Reg. Environ. Change, 19(5), 1387-1399, doi:10.1007/s10113-018-1385-8.

Huggel, C. et al., 2015b: A framework for the science contribution in climate adaptation: Experiences from science-policy processes in the Andes. Environ. Sci. Policy, 47, 80-94, doi:10.1016/j.envsci.2014.11.007.

Huggel, C. et al., 2005: The 2002 rock/ice avalanche at Kolka/Karmadon, Russian Caucasus: assessment of extraordinary avalanche formation and mobility, and application of QuickBird satellite imagery. Nat. Hazard. Earth Sys., 5(2), 173-187, doi:10.5194/nhess-5-173-2005.

Hummel, S., 2017: Relative water scarcity and country relations along cross-boundary rivers: Evidence from the Aral Sea basin. International Studies Quarterly, 61(4), 795-808, doi:10.1093/isq/sqx043.

Huntington, H.P. et al., 2017: How small communities respond to environmental change: patterns from tropical to polar ecosystems. Ecol. Soc. 22(3), 9.

Huss, M. and M. Fischer, 2016: Sensitivity of very small glaciers in the Swiss Alps to future climate change. Front. Earth Sci., 4, 34, doi:10.3389/ feart.2016.00034.

Huss, M. and R. Hock, 2015: A new model for global glacier change and sea-level rise. Front. Earth Sci, 3, 54, doi:10.3389/feart.2015.00054.

Huss, M. and R. Hock, 2018: Global-scale hydrological response to future glacier mass loss. Nat. Clim. Change, 8(2), 135-140, doi:10.1038/s41558017-0049-x.

Hussain, A. et al., 2018: Climate change-induced hazards and local adaptations in agriculture: a study from Koshi River Basin, Nepal. Nat. Hazards, 91(3), 1365-1383, doi:10.1007/s11069-018-3187-1.

ICIMOD, 2011: Glacial Lakes and Glacial Lake Outburst Floods in Nepal. ICIMOD, Kathmandu. [Available at: http://lib.icimod.org/record/27755]. Accessed 06/08/2019.

IHA, 2018: Hydropower status report 2018. International Hydropower Assocation, Sutton, United Kingdom. www.hydropower.org/publications/ 2018-hydropower-status-report. Accessed 06/08/2019.

IHCAP, 2017: Mountain and Lowland Linkages: A Climate Change Perspective in the Himalayas. Indian Himalayas Climate Adaptation Programme (IHCAP). [Available at: http://ihcap.in/?media_dl=872]. Accessed 06/08/2019.

Ikeda, A. and N. Matsuoka, 2002: Degradation of talus-derived rock glaciers in the upper engadin, Swiss alps. Permafrost Periglac., 13(2), 145-161, doi:10.1002/ppp.413.

II Jeong, D. and L. Sushama, 2018: Rain-on-snow events over North America based on two Canadian regional climate models. Clim. Dyn., 50(1-2), 303-316, doi:10.1007/s00382-017-3609-x.

Ilyashuk, B.P. et al., 2018: Rock glaciers in crystalline catchments: Hidden permafrost-related threats to alpine headwater lakes. Glob. Change Biol, 24(4), 1548-1562, doi:10.1111/gcb.13985.

Imperio, S., R. Bionda, R. Viterbi and A. Provenzale, 2013: Climate Change and Human Disturbance Can Lead to Local Extinction of Alpine Rock Ptarmigan: New Insight from the Western Italian Alps. PLOS ONE, 8(11), doi:10.1371/journal.pone.0081598.
Ingty, T., 2017: High mountain communities and climate change: adaptation, traditional ecological knowledge, and institutions. Clim. Change, 145(1-2), 41-55, doi:10.1007/s10584-017-2080-3.

IPCC, 2018: Summary for Policymakers. In: Global Warming of $1.5^{\circ} \mathrm{C}$. An IPCC Special Report on the impacts of global warming of $1.5^{\circ} \mathrm{C}$ above pre-industrial levels and related global greenhouse gas emission pathways, in the context of strengthening the global response to the threat of climate change, sustainable development, and efforts to eradicate poverty [Masson-Delmotte, V., P. Zhai, H. Pörtner, D. Roberts, J. Skea, P. Shukla, A. Pirani, W. Moufouma-Okia, C. Péan, R. Pidcock, S. Connors, J.B.R. Matthews, Y. Chen, X. Zhou, M.I. Gomis, E. Lonnoy, Maycock, M. Tignor and T. Waterfield (eds.)]. World Meteorological Organization, Geneva, Switzerland, 32 pp.

Isaak, D.J. et al., 2016: Slow climate velocities of mountain streams portend their role as refugia for cold-water biodiversity. PNAS, 113(16), 43744379, doi:10.1073/pnas.1522429113.

Isaksen, K., P. Holmlund, J.L. Sollid and C. Harris, 2001: Three deep alpine-permafrost boreholes in Svalbard and Scandinavia. Permafrost Periglac., 12(1), 13-25, doi:10.1002/ppp.380.

Isaksen, K. et al., 2011: Degrading Mountain Permafrost in Southern Norway: Spatial and Temporal Variability of Mean Ground Temperatures, 19992009. Permafrost Periglac., 22, 361-377, doi:10.1002/ppp.728.

Isaksen, K., J.L. Sollid, P. Holmlund and C. Harris, 2007: Recent warming of mountain permafrost in Svalbard and Scandinavia. J. Geophys. Res-Earth., 112(2), 235, doi:10.1029/2006JF000522.

Islam, S.U., S.J. Déry and A.T. Werner, 2017: Future climate change impacts on snow and water resources of the Fraser River Basin, British Columbia. J. Hydrometeorol., 18(2), 473-496, doi:10.1175/JHM-D-16-0012.1.

Ives, J.D., R.B. Shrestha and P.K. Mool, 2010: Formation of glacial lakes in the Hindu Kush-Himalayas and GLOF risk assessment. ICIMOD, Kathmandu. www.unisdr.org/files/14048_ICIMODGLOF.pdf. Accessed 06/08/2019.

Jackson, M. and G. Ragulina, 2014: Inventory of glacier-related hazardous events in Norway. Report no. 83 - 2014. Norwegian Water Resources and Energy Directorate. NVE, Oslo. [Available at: http://asp.bibliotekservice.no/ nve/title.aspx?tkey=22514]. Accessed 06/08/2019.

Jacob, D. et al., 2014: EURO-CORDEX: New high-resolution climate change projections for European impact research. Reg. Environ. Change, doi:10.1007/s10113-013-0499-2.

Jacobsen, D. et al., 2014: Runoff and the longitudinal distribution of macroinvertebrates in a glacier-fed stream: implications for the effects of global warming. Freshw. Biol., 59(10), 2038--2050, doi:10.1111/ fwb. 12405 .

Jacobsen, D., A.M. Milner, L.E. Brown and O. Dangles, 2012: Biodiversity under threat in glacier-fed river systems. Nat. Clim. Change, 2(5), 361-364, doi:10.1038/nclimate1435.

Jalilov, S.-M., S.A. Amer and F.A. Ward, 2018: Managing the water-energyfood nexus: Opportunities in Central Asia. J. Hydrol., 557, 407-425, doi:10.1016/j.jhydrol.2017.12.040.

Jamieson, S.S.R., M.W. Ewertowski and D.J.A. Evans, 2015: Rapid advance of two mountain glaciers in response to mine-related debris loading. J. Geophys. Res-Earth., 120(7), 1418-1435, doi:10.1002/2015JF003504.

Jamir, 0., 2016: Understanding India-Pakistan water politics since the signing of the Indus Water Treaty. Water Policy, 18(5), 1070-1087, doi:10.2166/ wp.2016.185.

Jenicek, M., J. Seibert and M. Staudinger, 2018: Modeling of future changes in seasonal snowpack and impacts on summer low flows in Alpine catchments. Water Resour. Res., 54, 538-556, doi:10.1002/2017WR021648.

Ji, Z.-M., 2016: Modeling black carbon and its potential radiative effects over the Tibetan Plateau. Adv. Clim. Change Res., 7(3), 139-144, doi:10.1016/J. ACCRE.2016.10.002.

Jin, H. et al., 2009: Changes in frozen ground in the source area of the Yellow River on the Qinghai-Tibet Plateau, China, and their eco-environmental impacts. Environ. Res. Lett., 4(4), doi:10.1088/1748-9326/4/4/045206. 
Johnston, A.N. et al., 2019: Ecological consequences of anomalies in atmospheric moisture and snowpack. Ecology, 100(4), doi:10.1002/ ecy.2638.

Johnstone, J.F. et al., 2016: Changing disturbance regimes, ecological memory, and forest resilience. Front. Ecol. Environ., 14(7), 369-378, doi:10.1002/ fee.1311.

Jomelli, V. et al., 2009: Impacts of future climatic change (2070-2099) on the potential occurrence of debris flows: A case study in the Massif des Ecrins (French Alps). Clim. Change, 97(1-2), 171-191, doi:10.1007/s10584-0099616-0.

Jones, B. and B.C. O'Neill, 2016: Spatially explicit global population scenarios consistent with the Shared Socioeconomic Pathways. Environ. Res. Lett., 11(2016), 084003, doi:10.1088/1748-9326/11/8/084003.

Jones, D.B., S. Harrison, K. Anderson and R.A. Betts, 2018: Mountain rock glaciers contain globally significant water stores. Sci. Rep., 8, 2834, doi:10.1038/s41598-018-21244-w.

Jordan, S. et al., 2016: Loss of genetic diversity and increased subdivision in an endemic Alpine stonefly threatened by climate change. PLOS ONE, 11(6), e0157386, doi:10.1371/journal.pone.0157386.

Joshi, S. et al., 2013: Herders' perceptions of and responses to climate change in Northern Pakistan. Environ. Manage., 52(3), 639-648, doi:10.1007/ s00267-013-0062-4.

Jost, G., R. Moore, B. Menounos and R. Wheate, 2012: Quantifying the contribution of glacier runoff to streamflow in the upper Columbia River Basin, Canada. Hydrol. Earth Syst. Sc., 16(3), 849-860, doi:10.5194/hess16-849-2012.

Jost, G. and F. Weber, 2013: Potential Impacts of Climate Change on BC Hydro's Water Resources. BC Hydro, Canada. www.bchydro.com/content/dam/ hydro/medialib/internet/documents/about/climate_change_report_2012. pdf. Accessed on 06/08/2019.

Jurt, C. et al., 2015: Local perceptions in climate change debates: insights from case studies in the Alps and the Andes. Clim. Change, 133(3), 511523, doi:10.1007/s10584-015-1529-5.

Kääb, A., R. Frauenfelder and I. Roer, 2007: On the response of rockglacier creep to surface temperature increase. Glob. Planet. Change, 56(1), 172187, doi:10.1016/j.gloplacha.2006.07.005.

Kääb, A. et al., 2018: Massive collapse of two glaciers in western Tibet in 2016 after surge-like instability. Nat. Geosci, 11(2), 114-120, doi:10.1038/ s41561-017-0039-7.

Kaenzig, R., 2015: Can glacial retreat lead to migration? A critical discussion of the impact of glacier shrinkage upon population mobility in the Bolivian Andes. Popul. Environ., 36(4), 480-496, doi:10.1007/s11111-014-0226-z.

Kaenzig, R., M. Rebetez and G. Serquet, 2016: Climate change adaptation of the tourism sector in the Bolivian Andes. Tourism Geographies, 18(2), 111-128, doi:10.1080/14616688.2016.1144642.

Kainz, M.J., R. Ptacnik, S. Rasconi and H.H. Hager, 2017: Irregular changes in lake surface water temperature and ice cover in subalpine Lake Lunz, Austria. Inland Waters, 7(1), 27-33, doi:10.1080/20442041.2017.1294332.

Kala, C.P., 2014: Deluge, disaster and development in Uttarakhand Himalayan region of India: Challenges and lessons for disaster management. Int. J. Dis. Risk. Re., 8, 143-152, doi:10.1016/j.ijdrr.2014.03.002.

Kapnick, S. and A. Hall, 2012: Causes of recent changes in western North American snowpack. Clim. Dyn., 38(9-10), 1885-1899, doi:10.1007/ s00382-011-1089-y.

Kapnick, S.B. and T.L. Delworth, 2013: Controls of global snow under a changed climate. J. Clim., 26(15), 5537-5562, doi:10.1175/JCLI-D-12-00528.1.

Kapnick, S.B. et al., 2014: Snowfall less sensitive to warming in Karakoram than in Himalayas due to a unique seasonal cycle. Nat. Geosci., 7(11), 834-840, doi:10.1038/ngeo2269.

Kargel, J.S. et al., 2016: Geomorphic and geologic controls of geohazards induced by Nepal's 2015 Gorkha earthquake. Science, 351(6269), aac8353, doi:10.1126/science.aac8353.
Kaspari, S. et al., 2014: Seasonal and elevational variations of black carbon and dust in snow and ice in the Solu-Khumbu, Nepal and estimated radiative forcings. Atmos. Chem. Phys., 14(15), 8089-8103, doi:10.5194/ acp-14-8089-2014.

Katsuyama, Y., M. Inatsu, K. Nakamura and S. Matoba, 2017: Global warming response of snowpack at mountain range in northern Japan estimated using multiple dynamically downscaled data. Cold Reg. Sci. Technol., 136, 62-71. doi:10.1016/j.coldregions.2017.01.006.

Keiler, M. and S. Fuchs, 2018: Challenges for natural hazard and risk management in mountain regions of Europe. In: Oxford Research Encyclopedia of Natural Hazard Science. Oxford University Press, Oxford. doi:10.1093/acrefore/9780199389407.013.322.

Kenner, R. et al., 2017: Factors controlling velocity variations at short-term, seasonal and multiyear time scales, Ritigraben Rock Glacier, western Swiss Alps. Permafrost Periglac., 28(4), 675-684, doi:10.1002/ppp.1953.

Keuschnig, M. et al., 2015: Permafrost-Related Mass Movements: Implications from a Rock Slide at the Kitzsteinhorn, Austria. In: Engineering Geology for Society and Territory, pp. 255-259. doi.org/10.1007/978-3-31909300-0_48.

Khadim, A.N., 2016: Defending glaciers in Argentina. Peace Review, 28(1), 65-75, doi:10.1080/10402659.2016.1130383.

Khamis, K., L.E. Brown, D.M. Hannah and A.M. Milner, 2015: Experimental evidence that predator range expansion modifies alpine stream community structure. Freshw. Sci. 34(1), 66-80, doi:10.1086/679484.

Khamis, K., L.E. Brown, D.M. Hannah and A.M. Milner, 2016: Glacier-groundwater stress gradients control alpine river biodiversity. Ecohydrology, 9(7), 1263-1275, doi:10.1002/eco.1724.

Khanal, N.R., J.-M. Hu and P. Mool, 2015: Glacial lake outburst flood risk in the Poiqu/Bhote Koshi/Sun Koshi river basin in the Central Himalayas. Mt. Res. Dev., 35(4), 351-364, doi:10.1659/MRD-JOURNAL-D-15-00009.

Kienholz, C. et al., 2017: Mass balance evolution of black rapids glacier, Alaska, 1980-2100, and its implications for surge recurrence. Front. Earth Sci., 5, 56, doi:10.3389/feart.2017.00056.

Kirkpatrick, J.B. et al., 2017: Causes and consequences of variation in snow incidence on the high mountains of Tasmania, 1983-2013. Aust. J. Bot., 65(3), 214-224, doi:10.1071/BT16179.

Kissel, A.M., W.J. Palen, M.E. Ryan and M.J. Adams, 2019: Compounding effects of climate change reduce population viability of a montane amphibian. Ecol. Appl., 29(2), e01832, doi:10.1002/eap.1832.

Kitzberger, T., D.A. Falk, A.L. Westerling and T.W. Swetnam, 2017: Direct and indirect climate controls predict heterogeneous early-mid 21st century wildfire burned area across western and boreal North America. PLOS ONE, 12(12), e0188486, doi:10.1371/journal.pone.0188486.

Knowles, J.F., N.P. Molotch, E. Trujillo and M.E. Litvak, 2018: Snowmelt-driven trade-offs between early and late season productivity negatively impact forest carbon uptake during drought. Geophys. Res. Lett., 45(7), 30873096, doi:10.1002/2017GL076504.

Konchar, K.M. et al., 2015: Adapting in the shadow of Annapurna: a climate tipping point. J. Ethnobiol., 35(3), 449-471, doi:10.2993/0278-077135.3.449.

Kormann, C., T. Francke, M. Renner and A. Bronstert, 2015: Attribution of high resolution streamflow trends in Western Austria - An approach based on climate and discharge station data. Hydrol. Earth Syst. Sc., 19(3), 12251245, doi:10.5194/hess-19-1225-2015.

Korup, O., T. Gorum and Y. Hayakawa, 2012: Without power? Landslide inventories in the face of climate change. Earth Surf. Process. Landf., 37(1), 92-99, doi:10.1002/esp.2248.

Kos, A. et al., 2016: Contemporary glacier retreat triggers a rapid landslide response, Great Aletsch Glacier, Switzerland. Geophys. Res. Lett., 43(24), 12466-12474, doi:10.1002/2016GL071708.

Kraaijenbrink, P.D.A., M.F.P. Bierkens, A.F. Lutz and W.W. Immerzeel, 2017: Impact of a global temperature rise of 1.5 degrees Celsius on Asia's glaciers. Nature, 549(7671), 257-260, doi:10.1038/nature23878. 
Krautblatter, M., D. Funk and F.K. Guenzel, 2013: Why permafrost rocks become unstable: a rock-ice-mechanical model in time and space. Earth Surf. Process. Landf., 38(8), 876-887, doi:10.1002/esp.3374.

Kriegel, D. et al., 2013: Changes in glacierisation, climate and runoff in the second half of the 20th century in the Naryn basin, Central Asia. Glob. Planet. Change, 110, 51-61, doi:10.1016/j.gloplacha.2013.05.014.

Kronenberg, J., 2013: Linking ecological economics and political ecology to study mining, glaciers and global warming. Environmental Policy and Governance, 23(2), 75-90, doi:10.1002/eet.1605.

Kropácek, J. et al., 2013: Analysis of ice phenology of lakes on the Tibetan Plateau from MODIS data. The Cryosphere, 7(1), 287-301, doi:10.5194/ tc-7-287-2013.

Kuang, X. and J.J. Jiao, 2016: Review on climate change on the Tibetan Plateau during the last half century. J. Geophys. Res-Atmos., 121(8), 3979-4007, doi:10.1002/2015JD024728.

Kulonen, K., C. Adler, C. Bracher and S. Wymann von Dach, 2019: Spatial context matters for monitoring and reporting on SDGs: Reflections based on research in mountain regions. GAIA, 28(2), 90-94. doi:10.14512/ gaia.28.2.5.

Kummert, M., R. Delaloye and L. Braillard, 2017: Erosion and sediment transfer processes at the front of rapidly moving rock glaciers: Systematic observations with automatic cameras in the western Swiss Alps. Permafrost Periglac., 29(1), 21-33, doi:10.1002/ppp.1960.

Lacelle, D., A. Brooker, R.H. Fraser and S.V. Kokelj, 2015: Distribution and growth of thaw slumps in the Richardson Mountains-Peel Plateau region, northwestern Canada. Geomorphology, 235, 40-51, doi:10.1016/j. geomorph.2015.01.024.

Lafaysse, M. et al., 2014: Internal variability and model uncertainty components in future hydrometeorological projections: The Alpine Durance basin. Water Resour. Res., 50(4), 3317-3341, doi:10.1002/2013WR014897.

Lamontagne-Hallé, P., J.M. McKenzie, B.L. Kurylyk and S.C. Zipper, 2018: Changing groundwater discharge dynamics in permafrost regions. Environ. Res. Lett, 13(8), 084017, doi:10.1088/1748-9326/aad404.

Lamprecht, A. et al., 2018: Climate change leads to accelerated transformation of high-elevation vegetation in the central Alps. New Phytologist, 220(2), 447-459, doi:10.1111/nph.15290.

Lane, S.N. et al., 2017: Sediment export, transient landscape response and catchment-scale connectivity following rapid climate warming and Alpine glacier recession. Geomorphology, 277, 210-227, doi:10.1016/j. geomorph.2016.02.015.

Langford, H., A.J. Hodson, S. Banwart and C.E. Bøggild, 2010: The microstructure and biogeochemistry of Arctic cryoconite granules. Ann. Glaciol, 51(56), 87-94, doi:10.3189/172756411795932083.

Larsen, C. et al., 2015: Surface melt dominates Alaska glacier mass balance. Geophys. Res. Lett., 42(14), 5902-5908, doi:10.1002/2015GL064349.

Lavigne, A., N. Eckert, L. Bel and E. Parent, 2015: Adding expert contributions to the spatiotemporal modelling of avalanche activity under different climatic influences. J.R. Stat. Soc. C-Appl., 64(4), 651-671, doi:10.1111/ rssc. 12095.

Lavoie, R.A. et al., 2013: Biomagnification of Mercury in Aquatic Food Webs: A Worldwide Meta-Analysis. Environ. Sci. Technol., 47(23), 13385-13394. doi:10.1021/es403103t.

Lawrimore, J.H. et al., 2011: An overview of the Global Historical Climatology Network monthly mean temperature data set, version 3. J. Geopyhs. Res., 116(D19), 1785, doi:10.1029/2011JD016187.

Lazar, B. and M. Williams, 2008: Climate change in western ski areas: Potential changes in the timing of wet avalanches and snow quality for the Aspen ski area in the years 2030 and 2100. Cold Reg. Sci. Technol., 51(2-3), 219228. doi:10.1016/j.coldregions.2007.03.015.

Leach, J.A. and R.D. Moore, 2014: Winter stream temperature in the rain-onsnow zone of the Pacific Northwest: influences of hillslope runoff and transient snow cover. Hydrol. Earth Syst. Sci., 18(2), 819-838, doi:10.5194/ hess-18-819-2014.
Lee, C.M., 2012: Withering snow and ice in the mid-latitudes: A new archaeological and paleobiological record for the Rocky Mountain region. Arctic, 65(5), 165-177, doi:10.14430/arctic4191.

Lee, S.-Y., A.F. Hamlet and E.E. Grossman, 2016: Impacts of climate change on regulated streamflow, hydrologic extremes, hydropower production, and sediment discharge in the Skagit river basin. Northwest Sci., 90(1), 23-43, doi:10.3955/046.090.0104.

Lencioni, V., 2018: Glacial influence and stream macroinvertebrate biodiversity under climate change: Lessons from the Southern Alps. Sci. Total Environ., 622, 563-575, doi:10.1016/j.scitotenv.2017.11.266.

Lencioni, V., O. Jousson, G. Guella and P. Bernabo, 2015: Cold adaptive potential of chironomids overwintering in a glacial stream. Physiol. Entomol., 40(1), 43-53, doi:10.1111/phen.12084.

Lesica, P., 2014: Arctic-Alpine plants decline over two decades in Glacier National Park, Montana, U.S.A. Arct. Antarct. Alp. Res., 46(2), 327-332, doi:10.1657/1938-4246-46.2.327.

Lesica, P. and E.E. Crone, 2016: Arctic and boreal plant species decline at their southern range limits in the Rocky Mountains. Ecol. Letters, 20(2), 166-174, doi:10.1111/ele.12718.

Li, C. et al., 2016: Sources of black carbon to the Himalayan-Tibetan Plateau glaciers. Nat. Commun., 7(1), 12574, doi:10.1038/ncomms12574.

Li, J. et al., 2017: Evidence for persistent organic pollutants released from melting glacier in the central Tibetan Plateau, China. Environ. Pollut., 220, 178-185, doi:10.1016/j.envpol.2016.09.037.

$\mathrm{Li}$, X. et al., 2018: Importance of mountain glaciers as a source of dissolved organic carbon. J. Geopyhs. Res. F- Earth Surface, 24(10), GB4033, doi:10.1029/2017JF004333.

Liang, Q. et al., 2018: Shifts in plant distributions in response to climate warming in a biodiversity hotspot, the Hengduan Mountains. J. Biogeogr., 45, 1334-1344, doi:10.1111/jbi.13229.

Lin, H. et al., 2017: A decreasing glacier mass balance gradient from the edge of the Upper Tarim Basin to the Karakoram during 2000-2014. Sci. Rep., 7(1), 6712, doi:10.1038/s41598-017-07133-8.

Lin, Z., J. Luo and F. Niu, 2016: Development of a thermokarst lake and its thermal effects on permafrost over nearly $10 \mathrm{yr}$ in the Beiluhe Basin, Qinghai-Tibet Plateau. Geosphere, 12(2), 632-643, doi:10.1130/ GES01194.1.

Linsbauer, A. et al., 2016: Modelling glacier-bed overdeepenings and possible future lakes for the glaciers in the Himalaya-Karakoram region. Ann. Glaciol., 57(71), 119-130, doi:10.3189/2016AoG71A627.

Littell, J.S., 2018: Drought and fire in the western USA: is climate attribution enough? Curr. Clim. Chang. Rep., 4(4), 396-406, doi:10.1007/s40641-0180109-y.

Littell, J.S., D. Mckenzie, H.Y. Wan and S.A. Cushman, 2018: Climate change and future wildfire in the western United States: an ecological approach to nonstationarity. Earth's Future, 6(8), 1097-1111, doi:10.1029/2018EF000878.

Liu, G. et al., 2017: Permafrost warming in the context of step-wise climate change in the Tien Shan Mountains, China. Permafrost Periglac., 28(1), 130-139, doi:10.1002/ppp.1885.

Liu, X., Z. Cheng, L. Yan and Z.-Y. Yin, 2009: Elevation dependency of recent and future minimum surface air temperature trends in the Tibetan Plateau and its surroundings. Glob. Planet. Change, 68(3), 164-174, doi:10.1016/j. gloplacha.2009.03.017.

Liu, Y. et al., 2016: Storage of dissolved organic carbon in Chinese glaciers. J. Glaciol., 62(232), 402-406, doi:10.1017/jog.2016.47.

Loriaux, T. and G. Casassa, 2013: Evolution of glacial lakes from the Northern Patagonia Icefield and terrestrial water storage in a sea-level rise context. Glob. Planet. Change, 102, 33-40, doi:10.1016/j.gloplacha.2012.12.012.

Lozny, L.R., 2013: Continuity and Change in Cultural Adaptation to Mountain Environments. Springer New York Heidelberg Dordrecht London, New York, $410 \mathrm{pp}$. 
Lu, Q., D. Zhao and S. Wu, 2017: Simulated responses of permafrost distribution to climate change on the Qinghai-Tibet Plateau. Sci. Rep., 7(1), 3845, doi:10.1038/s41598-017-04140-7.

Luethi, R., S. Gruber and L. Ravanel, 2015: Modelling transient ground surface temperatures of past rockfall events: Towards a better understanding of failure mechanisms in changing periglacial environments. Geografiska Annaler. Series A, Physical Geography, 97(4), 753-767, doi:10.1111/ geoa.12114.

Luethi, R., M. Phillips and M. Lehning, 2017: Estimating non-conductive heat flow leading to intra-permafrost talik formation at the Ritigraben Rock Glacier (Western Swiss Alps). Permafrost Periglac., 28(1), 183-194, doi:10.1002/ppp.1911.

Lugon, R. and M. Stoffel, 2010: Rock-glacier dynamics and magnitudefrequency relations of debris flows in a high-elevation watershed: Ritigraben, Swiss Alps. Glob. Planet. Change, 73(3), 202-210, doi:10.1016/j. gloplacha.2010.06.004.

Lutz, A. et al., 2016: Climate change impacts on the upper Indus hydrology: Sources, shifts and extremes. PLOS ONE, 11(11), e0165630, doi:10.1371/ journal.pone.0165630.

Lynch, B.D., 2012: Vulnerabilities, competition and rights in a context of climate change toward equitable water governance in Peru's Rio Santa Valley. Glob. Environ. Change., 22(2), 364-373, doi:10.1016/j. gloenvcha.2012.02.002.

Macfarlane, A., 1976: Resources and population: A study of the Gurungs of Nepal. Cambridge University Press, Cambridge, 384 pp. ISBN 101107406862.

Mackintosh, A.N. et al., 2017: Regional cooling caused recent New Zealand glacier advances in a period of global warming. Nat. Commun., 8, 14202, doi:10.1038/ncomms14202.

Madani, K. and J.R. Lund, 2010: Estimated impacts of climate warming on California's high-elevation hydropower. Clim. Change, 102(3-4), 521-538, doi:10.1007/s10584-009-9750-8.

Magnin, F. et al., 2015: Thermal characteristics of permafrost in the steep alpine rock walls of the Aiguille du Midi (Mont Blanc Massif, $3842 \mathrm{~m}$ a.s.l). The Cryosphere, 9(1), 109-121, doi:10.5194/tc-9-109-2015.

Magnin, F. et al., 2017: Modelling rock wall permafrost degradation in the Mont Blanc massif from the LIA to the end of the 21st century. The Cryosphere, 11(4), 1813-1834, doi:10.5194/tc-11-1813-2017.

Mahoney, P.J. et al., 2018: Navigating snowscapes: scale-dependent responses of mountain sheep to snowpack properties. Ecol. Appl., 28(7), 1715-1729, doi:10.1002/eap.1773.

Mallory, C.D. and M.S. Boyce, 2018: Observed and predicted effects of climate change on Arctic caribou and reindeer. Environ. Rev., 26, 13-25, doi:10.1139/er-2017-0032.

Mamet, S.D. et al., 2017: Recent increases in permafrost thaw rates and areal loss of palsas in the western Northwest Territories, Canada. Permafrost Periglac., 28(4), 619-633, doi:10.1002/ppp.1951.

Mankin, J.S. and N.S. Diffenbaugh, 2015: Influence of temperature and precipitation variability on near-term snow trends. Clim. Dyn., 45(3-4), 1099-1116, doi:10.1007/s00382-014-2357-4.

Mark, B.G. et al., 2010: Climate change and tropical Andean glacier recession: Evaluating hydrologic changes and livelihood vulnerability in the Cordillera Blanca, Peru. Ann. Am. Assoc. Geogr., 100(4), 794-805, doi:10.1080/0004 5608.2010.497369.

Mark, B.G. et al., 2017: Glacier loss and hydro-social risks in the Peruvian Andes. Glob. Planet. Change, 159, 61-76, doi:10.1016/j.gloplacha.2017.10.003.

Marke, T. et al., 2015: Scenarios of future snow conditions in Styria (Austrian Alps). J. Hydrometeorol., 16(1), 261-277, doi:10.1175/JHM-D-14-0035.1.

Marmy, A. et al., 2016: Semi-automated calibration method for modelling of mountain permafrost evolution in Switzerland. The Cryosphere, 10(6), 2693-2719, doi:10.5194/tc-10-2693-2016.
Marmy, A., N. Salzmann, M. Scherler and C. Hauck, 2013: Permafrost model sensitivity to seasonal climatic changes and extreme events in mountainous regions. Environ. Res. Lett., 8(3), 035048, doi:10.1088/17489326/8/3/035048.

Marston, R.A., 2008: Land, life, and environmental change in mountains. Ann. Am. Assoc. Geogr., 98(3), 507-520, doi:10.1080/00045600802118491.

Marty, C., A.-M. Tilg and T. Jonas, 2017: Recent evidence of large-scale receding snow water equivalents in the European Alps. J. Hydrometeorol., 18(4), 1021-1031, doi:10.1175/JHM-D-16-0188.1.

Marzeion, B., A.H. Jarosch and J.M. Gregory, 2014: Feedbacks and mechanisms affecting the global sensitivity of glaciers to climate change. The Cryosphere, 8(1), 59-71, doi:10.5194/tc-8-59-2014.

Marzeion, B., A.H. Jarosch and M. Hofer, 2012: Past and future sea-level change from the surface mass balance of glaciers. The Cryosphere, 6(6), 1295-1322, doi:10.5194/tc-6-1295-2012.

Marzeion, B., G. Kaser, F. Maussion and N. Champollion, 2018: Limited influence of climate change mitigation on short-term glacier mass loss. Nat. Clim. Change, 8(4), 305-308, doi:10.1038/s41558-018-0093-1.

Matteodo, M., K. Ammann, E.P. Verrecchia and P. Vittoz, 2016: Snowbeds are more affected than other subalpine-alpine plant communities by climate change in the Swiss Alps. Ecol. Evol., 6(19), 6969-6982, doi:10.1002/ ece3.2354.

Matthews, J.A. and A.E. Vater, 2015: Pioneer zone geo-ecological change: Observations from a chronosequence on the Storbreen glacier foreland, Jotunheimen, southern Norway. Catena, 135, 219-230, doi:10.1016/j. catena.2015.07.016.

Maxwell, J.D., A. Call and S.B. St Clair, 2019: Wildfire and topography impacts on snow accumulation and retention in montane forests. For. Ecol. Manage., 432, 256-263, doi:10.1016/j.foreco.2018.09.021.

McCabe, G.J. et al., 2007: Rain-on-snow events in the Western United States. Bull. Am. Meterol. Soc., 88(3), 319-328, doi:10.1175/BAMS-88-3-319.

McClung, D.M., 2013: The effects of El Niño and La Niña on snow and avalanche patterns in British Columbia, Canada, and central Chile. J. Glaciol., 59(216), 783-792, doi:10.3189/2013JoG12J192.

McColl, S.T., 2012: Paraglacial rock-slope stability. Geomorphology, 153154, 1-16, doi:10.1016/j.geomorph.2012.02.015.

McDonald, K.I., 1989: Impacts of glacier-related landslides on the settlement at Hopar, Karakoram Himalaya. Ann. Glaciol., 13, 185-188, doi:10.3189/ S0260305500007862.

McDowell, G. et al., 2013: Climate-related hydrological change and human vulnerability in remote mountain regions: a case study from Khumbu, Nepal. Reg. Environ. Change, 13(2), 299-310, doi:10.1007/s10113-012-0333-2.

McDowell, G. etal., 2019:Adaptation action and research in glaciated mountain systems: Are they enough to meet the challenge of climate change? Glob. Environ. Change, 54, 19-30, doi:10.1016/j.gloenvcha.2018.10.012.

McDowell, G. and M.N. Koppes, 2017: Robust adaptation research in high mountains: Integrating the scientific, social, and ecological dimensions of glacio-hydrological change. Water, 9(10), doi:10.3390/w9100739.

McDowell, G., E. Stephenson and J. Ford, 2014: Adaptation to climate change in glaciated mountain regions. Clim. Change, 126(1-2), 77-91, doi:10.1007/s10584-014-1215-z.

Mcdowell, N.G. et al., 2018: Predicting chronic climate-driven disturbances and their mitigation. Trends Ecol. Evol., 33(1), 15-27, doi:10.1016/j. tree.2017.10.002.

McNabb, R.W. and R. Hock, 2014: Alaska tidewater glacier terminus positions, 1948-2012. J. Geopyhs. Res.-Earth Surface, 119(2), 153-167, doi:10.1002/2013JF002915.

McNabb, R.W., R. Hock and M. Huss, 2015: Variations in Alaska tidewater glacier frontal ablation, 1985-2013. J. Geophys. Res-Earth., 120(1), 120136. doi:10.1002/2014JF003276.

McNeeley, S.M., 2017: Sustainable climate change adaptation in Indian Country. Weather Climate and Society, 9(3), 392-403, doi:10.1175/ wcas-d-16-0121.1. 
Mearns, L. et al., 2017: The NA-CORDEX dataset, version 1.0. NCAR Climate Data Gateway. Boulder, Colourado: doi:10.5065/D6SJ1JCH. Accessed 06/08/2019.

Medwedeff, W.G. and G.H. Roe, 2017: Trends and variability in the global dataset of glacier mass balance. Clim. Dyn., 48(9-10), 3085-3097, doi:10.1007/s00382-016-3253-x.

Ménégoz, M. et al., 2014: Snow cover sensitivity to black carbon deposition in the Himalayas: from atmospheric and ice core measurements to regional climate simulations. Atmos. Chem. Phys., 14(8), 4237-4249, doi:10.5194/ acp-14-4237-2014.

Menounos, B. et al., 2013: Did rock avalanche deposits modulate the late Holocene advance of Tiedemann Glacier, southern Coast Mountains, British Columbia, Canada? Earth Planet. Sci. Lett., 384, 154-164, doi:10.1016/j. epsl.2013.10.008.

Menounos, B. et al., 2019: Heterogeneous changes in western north american glaciers linked to decadal variability in zonal wind strength. Geophys. Res. Lett., 46(1), 200-209, doi:10.1029/2018GL080942.

Merkle, J.A. et al., 2016: Large herbivores surf waves of green-up during spring. Proc. R. Soc. B-Biol. Sci, 283(1833), doi:10.1098/rspb.2016.0456.

Mernild, S.H. et al., 2013: Global glacier changes: a revised assessment of committed mass losses and sampling uncertainties. The Cryosphere, 7(5), 1565-1577, doi:10.5194/tc-7-1565-2013.

Merrey, D.J. et al., 2018: Evolving high altitude livelihoods and climate change: a study from Rasuwa District, Nepal. Food Security, 10(4), 10551071, doi:10.1007/s12571-018-0827-y.

Middleton, A.D. et al., 2018: Green-wave surfing increases fat gain in a migratory ungulate. Oikos, 127(7), 1060-1068, doi:10.1111/oik.05227.

Milan, A. and R. Ho, 2014: Livelihood and migration patterns at different altitudes in the Central Highlands of Peru. Clim. Dev., 6(1), 69-76, doi:10. 1080/17565529.2013.826127.

Millan, R., J. Mouginot and E. Rignot, 2017: Mass budget of the glaciers and ice caps of the Queen Elizabeth Islands, Canada, from 1991 to 2015. Environ. Res. Lett., 12(2), 024016, doi:10.1088/1748-9326/aa5b04.

Millar, C.I. and N.L. Stephenson, 2015: Temperate forest health in an era of emerging megadisturbance. Science, 349 (6250), 823-826, doi:10.1126/ science.aaa9933.

Mills, L.S. et al., 2018: Winter colour polymorphisms identify global hot spots for evolutionary rescue from climate change. Science, 359(6379), 10331036, doi:10.1126/science.aan8097.

Milner, A.M. et al., 2017: Glacier shrinkage driving global changes in downstream systems. PNAS, 114(37), 9770-9778, doi:10.1073/ pnas. 1619807114.

Minder, J.R., T.W. Letcher and C. Liu, 2018: The character and causes of elevation-dependent warming in high-resolution simulations of Rocky Mountain climate change. J. Clim., 31(6), 2093-2113, doi:10.1175/ JCLI-D-17-0321.1.

Minville, M., S. Krau, F. Brissette and R. Leconte, 2010: Behaviour and performance of a water resource system in Québec (Canada) under adapted operating policies in a climate change context. Water Resour. Manage., 24(7), 1333-1352, doi:10.1007/s11269-009-9500-8.

Mishra, A. et al., 2019: Adaptation to climate change in the Hindu Kush Himalaya: Stronger action urgently needed. In: The Hindu Kush Himalaya Assessment: Mountains, Climate Change, Sustainability and People [Wester, P., A. Mishra, A. Mukherji and A.B. Shrestha (eds.)]. Springer International Publishing, Cham, 457-490.

Mock, C.J., K.C. Carter and K.W. Birkeland, 2017: Some Perspectives on Avalanche Climatology. A. Assoc. Am. Geog., 107(2), 299-308, doi:10.108 0/24694452.2016.1203285.

Molden, D.J. et al., 2014: Water infrastructure for the Hindu Kush Himalayas. Int. J. Water Resour. D., 30(1), 60-77, doi:10.1080/07900627.2013.859044.

Molina, L.T. et al., 2015: Pollution and its Impacts on the South American Cryosphere. Earth's Future, 3, 345-369, doi:10.1002/2015EF000311.
Montana, E., H.P. Diaz and M. Hurlbert, 2016: Development, local livelihoods, and vulnerabilities to global environmental change in the South American Dry Andes. Reg. Environ. Change, 16(8), 2215-2228, doi:10.1007/s10113015-0888-9.

Moran-Tejéda, E., J.I. López-Moreno, M. Stoffel and M. Beniston, 2016: Rain-on-snow events in Switzerland: recent observations and projections for the 21st century. Clim. Res., 71(2), 111-125, doi:10.3354/cr01435.

Morueta-Holme, N. et al., 2015: Strong upslope shifts in Chimborazo's vegetation over two centuries since Humboldt. PNAS, 112(41), 1274112745, doi:10.1073/pnas.1509938112.

Mouginot, J. and E. Rignot, 2015: Ice motion of the Patagonian Icefields of South America: 1984-2014. Geophys. Res. Lett., 42, 1441-1449, doi:10.1002/2014GL062661.

Mourey, J., M. Marcuzzi., L. Ravanel. and F. Pallandre., 2019: Effects of climate change on high Alpine environments: the evolution of mountaineering routes in the Mont Blanc massif (Western Alps) over half a century. Arct. Antarct. Alp. Res. 51(1), 176-189, doi:10.1080/15230430.2019.1612216.

Mourey, J. and L. Ravanel, 2017: Evolution of access routes to high mountain refuges of the Mer de Glace Basin (Mont Blanc Massif, France). Revue de Géographie Alpine, 105(4), doi:10.4000/rga.3790.

Moyer, A.N., R.D. Moore and M.N. Koppes, 2016: Streamflow response to the rapid retreat of a lake-calving glacier. Hydrol. Process. , 30(20), 3650-3665, doi:10.1002/hyp.10890.

Mu, C. et al., 2017: Relict Mountain Permafrost Area (Loess Plateau, China) Exhibits High Ecosystem Respiration Rates and Accelerating Rates in Response to Warming. J. Geophys. Res-Biogeo, 122(10), 2580-2592, doi:10.1002/2017JG004060.

$\mathrm{Mu}, \mathrm{C}$. et al., 2016: Carbon loss and chemical changes from permafrost collapse in the northern Tibetan Plateau. J. Geophys. Res-Biogeo., 121(7), 1781-1791, doi:10.1002/2015JG003235.

Muellner-Riehl, A.N., 2019: Mountains as evolutionary arenas: Patterns, emerging approaches, paradigm shifts, and their implications for plant phylogeographic research in the Tibeto-Himalayan Region. Front. Plant. Sci., 10, 1-18, doi:10.3389/fpls.2019.00195.

Mukherji, A. et al., 2019: Contributions of the cryosphere to mountain communities in the Hindu Kush Himalaya: a review. Reg. Environ. Change, 42(2), 228, doi:10.1007/s10113-019-01484-w.

Mukhopadhyay, B. and A. Khan, 2014: Rising river flows and glacial mass balance in central Karakoram. J. Hydrol., 513, 192-203, doi:10.1016/j. jhydrol.2014.03.042.

Muñoz, R. et al., 2016: Managing glacier related risks disaster in the Chucchún Catchment, Cordillera Blanca, Peru. In: Climate Change Adaption Strategies - An upstream-downstream perspective [Salzmann, N., C. Huggel, S.U. Nussbaumer and G. Ziervogel (eds.)]. Springer International Publishing, Switzerland, 59-78.

Murphy, S.F. et al., 2018: Fire, flood, and drought: extreme climate events alter flow paths and stream chemistry. J. Geophys. Res.-Biogeosci., 123(8), 2513-2526, doi:10.1029/2017JG004349.

Musselman, K.N. et al., 2018: Projected increases and shifts in rain-on-snow flood risk over western North America. Nat. Clim. Change, 8(9), 808-812, doi:10.1038/s41558-018-0236-4.

Naaim, M., Y. Durand, N. Eckert and G. Chambon, 2013: Dense avalanche friction coefficients: influence of physical properties of snow. J. Glaciol., 59(216), 771-782, doi:10.3189/2013JoG12J205.

Naaim, M. et al., 2016: Impact of climate warming on avalanche activity in French Alps and increase of proportion of wet snow avalanches. Houille Blanche, 59(6), 12-20, doi:10.1051//hb/2016055.

Nagorski, S.A. et al., 2014: Spatial distribution of mercury in southeastern Alaskan streams influenced by glaciers, wetlands, and salmon. Environ. Pollut., 184, 62-72, doi:10.1016/j.envpol.2013.07.040.

Naiman, Z. et al., 2017: Impact of Mountains on Tropical Circulation in Two Earth System Models. J. Clim., 30(11), 4149-4163, doi:10.1175/ JCLI-D-16-0512.1. 
Najafi, M.R., F. Zwiers and N. Gillett, 2017: Attribution of the observed spring snowpack decline in British Columbia to anthropogenic climate change. J. Clim. 30, 4113-4130, doi:10.1175/JCLI-D-16-0189.1.

Namgay, K., J.E. Millar, R.S. Black and T. Samdup, 2014: Changes in Transhumant Agro-pastoralism in Bhutan: A Disappearing Livelihood? Hum. Ecol., 42(5), 779-792, doi:10.1007/s10745-014-9684-2.

Narama, C. et al., 2017: Seasonal drainage of supraglacial lakes on debris-covered glaciers in the Tien Shan Mountains, Central Asia. Geomorphology, 286, 133-142, doi:10.1016/j.geomorph.2017.03.002.

Navarro, F., H. Andrés, F. Acuña and F. José, 2018: Glaciares rocosos en la zona semiárida de Chile: relevancia de un recurso hídrico sin protección normativa. Cuadernos de Geografía: Revista Colombiana de Geografía, 27(2), 338-355, doi:10.15446/rcdg.v27n2.63370.

Nepal, S., 2016: Impacts of climate change on the hydrological regime of the Koshi river basin in the Himalayan region. Journal of Hydro-Environment Research, 10, 76-89, doi:10.1016/j.jher.2015.12.001.

Niedrist, G.H. et al., 2018: Climate warming increases vertical and seasonal water temperature differences and inter-annual variability in a mountain lake. Clim. Change, 151(3-4), 473-490, doi:10.1007/s10584-018-2328-6.

Nilsson, J., L.S. Sørensen, V.R. Barletta and R. Forsberg, 2015: Mass changes in Arctic ice caps and glaciers: implications of regionalizing elevation changes. The Cryosphere, 9, 139-150, doi:10.5194/tc-9-139-2015.

Nitu, R. et al., 2018: WMO Solid Precipitation Intercomparison Experiment (SPICE) (2012 - 2015). Instruments and Observing Methods Report, 131, World Meteorological Organization, Geneva. www.wmo.int/pages/prog/ www/IMOP/publications-IOM-series.html

Niu, F. et al., 2012: Development and thermal regime of a thaw slump in the Qinghai-Tibet plateau. Cold Reg. Sci. Technol., 83-84, 131-138. doi:10.1016/j.coldregions.2012.07.007.

Noël, B. et al., 2017: A tipping point in refreezing accelerates mass loss of Greenland's glaciers and ice caps. Nat. Commun., 8, 14730, doi:10.1038/ ncomms14730.

Noël, B. et al., 2018: Six decades of glacial mass loss in the Canadian Arctic Archipelago. J. Geophys. Res-Earth, 123(6), 1430-1449, doi:10.1029/2017JF004304.

Noetzli, J. et al., 2018: Permafrost thermal state [in "State of the Climate in 2017"]. Bull. Am. Meterol. Soc.

Noetzli, J. and S. Gruber, 2009: Transient thermal effects in Alpine permafrost. The Cryosphere, 3(1), 85-99, doi:10.5194/tc-3-85-2009.

Nothiger, C. and H. Elsasser, 2004: Natural hazards and tourism: New findings on the European Alps. Mt. Res. Dev., 24(1), 24-27. doi:10.1659/02764741(2004)024[0024:NHATNF]2.0.C0;2.

Nüsser, M. and R. Baghel, 2016: Local knowledge and global concerns: Artificial glaciers as a focus of environmental knowledge and development interventions. [Meusburger, P., T. Freytag, T., and L. Suarsana (eds.)]. Ethnic and Cultural Dimensions of Knowledge, 8. Springer, Cham, Switzerland, 191-209. ISBN: 978-3-319-21899-1. doi:10.1007/978-3-319-21900-4.

Nüsser, M. et al., 2018: Socio-hydrology of "artificial glaciers" in Ladakh, India: assessing adaptive strategies in a changing cryosphere. Reg. Environ. Change, 48(2), 1-11, doi:10.1007/s10113-018-1372-0.

Nüsser, M. and S. Schmidt, 2017: Nanga Parbat Revisited: Evolution and Dynamics of Sociohydrological Interactions in the Northwestern Himalaya. A. Assoc. Am. Geog., 107(2), 403-415, doi:10.1080/24694452.2016.1235495.

Nüsser, M., S. Schmidt and J. Dame, 2012: Irrigation and development in the upper indus Basin: Characteristics and recent changes of a socio-hydrological system in central Ladakh, India. Mt. Res. Dev., 32(1), 51-61, doi:10.1659/MRD-JOURNAL-D-11-00091.1.

Nuth, C. et al., 2019: Dynamic vulnerability revealed in the collapse of an Arctic tidewater glacier. Sci. Rep., 9(1), 5541, doi:10.1038/s41598-01941117-0.
Nyima, Y. and K.A. Hopping, 2019: Tibetan lake expansion from a pastoral perspective: Local observations and coping strategies for a changing environment. Society \& Natural Resources, 32(9), 965-982,

doi:10.1080/08941920.2019.1590667

O'Gorman, P.A., 2014: Contrasting responses of mean and extreme snowfall to climate change. Nature, 512(7515), 416-418, doi:10.1038/nature13625.

Obu, J. et al., 2019: Northern Hemisphere permafrost map based on TTOP modelling for 2000-2016 at $1 \mathrm{~km}^{2}$ scale. Earth-Sci. Rev., 193, 299-316, doi:j.earscirev.2019.04.023.

Ohmura, A., 2012: Enhanced temperature variability in high-altitude climate change. Theor. Appl. Climatol., 110(4), 499-508, doi:10.1007/s00704-0120687-x.

Oliva, M. and J. Ruiz-Fernández, 2015: Coupling patterns between para-glacial and permafrost degradation responses in Antarctica. Earth Surf. Process. Landf., 40(9), 1227-1238, doi:10.1002/esp.3716.

Oliver-Smith, A., 1979: Yungay avalanche of 1970 - Anthropological perspectives on disaster and social-change. Disasters, 3(1), 95-101, doi:10.1111/j.1467-7717.1979.tb00205.x.

Oliver-Smith, A., 1996: Anthropological research on hazards and disasters. Annual Review of Anthropology, 25, 303-328, doi:10.1146/annurev. anthro.25.1.303.

Oliver-Smith, A., 2014: Climate Change Adaptation and Disaster Risk Reduction in Highland Peru. [Glavovic, B.C. and G.P. Smith (eds.)]. Adapting to Climate Change: Lessons from Natural Hazards Planning.

Springer Netherlands, Dordrecht, 77-100.

Orlove, B. et al., 2019: Framing climate change in frontline communities: anthropological insights on how mountain dwellers in the USA, Peru, and Italy adapt to glacier retreat. Reg. Environ. Change, 19(5), 1295-1309, doi:10.1007/s10113-019-01482-y.

Østby, T.I. et al., 2017: Diagnosing the decline in climatic mass balance of glaciers in Svalbard over 1957-2014. The Cryosphere, 11, 191-215, doi:10.5194/tc-11-191-2017.

Outdoor Industry Association, 2017: The outdoor recreation economy. 20 p. [Available at: https://outdoorindustry.org/resource/2017-outdoor-recreatio n-economy-report/].

Oyler, J.W. et al., 2015: Artificial amplification of warming trends across the mountains of the western United States. Geophys. Res. Lett., 42(1), 153161, doi:10.1002/2014GL062803.

Paden, R., L.K. Harmon, C.R. Milling and T.U.o.N.T. Center for Environmental Philosophy, 2013: Philosophical Histories of the Aesthetics of Nature. Environmental Ethics, 35(1), 57-77, doi:10.5840/enviroethics20133516.

Pagán, B.R. et al., 2016: Extreme hydrological changes in the southwestern US drive reductions in water supply to Southern California by mid century. Environ. Res. Lett., 11, 1-11, doi:10.1088/1748-9326/11/9/094026.

Painter, T.H. et al., 2013: End of the Little Ice Age in the Alps forced by industrial black carbon. PNAS, 110(38), 15216-15221, doi:10.1073/ pnas. 1302570110.

Painter, T.H. et al., 2018: Variation in rising limb of Colorado River snowmelt runoff hydrograph controlled by dust radiative forcing in snow. Geophys. Res. Lett., 45(2), 797-808, doi:10.1002/2017GL075826.

Palazzi, E., L. Mortarini, S. Terzago and J. von Hardenberg, 2019: Elevation-dependent warming in global climate model simulations at high spatial resolution. Clim. Dyn., 52(5-6), 2685-2702, doi:10.1007/s00382018-4287-z.

Palazzi, E., J. von Hardenberg and A. Provenzale, 2013: Precipitation in the Hindu-Kush Karakoram Himalaya: Observations and future scenarios. J. Geophys. Res.-Atmos., 118(1), 85-100, doi:10.1029/2012JD018697.

Palazzi, E.L., L. Filippi and J.v. Hardenberg, 2017: Insights into elevation-dependent warming in the Tibetan Plateau-Himalayas from CMIP5 model simulations. Clim. Dyn., 48(11-12), 3991-4008, doi:10.1007/ s00382-016-3316-z. 
Palomo, I., 2017: Climate change impacts on ecosystem services in high mountain areas: A literature review. Mt. Res. Dev., 37(2), 179-187, doi:10.1659/mrd-journal-d-16-00110.1.

Panday, P.K., J. Thibeault and K.E. Frey, 2015: Changing temperature and precipitation extremes in the Hindu Kush-Himalayan region: an analysis of CMIP3 and CMIP5 simulations and projections. Int. J. Climatol., 35(10), 3058-3077, doi:10.1002/joc.4192.

Panetta, A.M., M.L. Stanton and J. Harte, 2018: Climate warming drives local extinction: Evidence from observation and experimentation. Science Advances, 4(2), eaaq1819, doi:10.1126/sciadv.aaq1819.

Papadaki, C. et al., 2016: Potential impacts of climate change on flow regime and fish habitat in mountain rivers of the south-western Balkans. Sci. Total Environ., 540, 418-428, doi:10.1016/j.scitotenv.2015.06.134.

Parveen, S., M. Winiger, S. Schmidt and M. Nüsser, 2015: Irrigation in Upper Hunza: Evolution of socio-hydrological interactions in the Karakoram, northern Pakistan. Erdkunde, 69(1), 69-85, doi:10.3112/ erdkunde.2015.01.05.

Pathak, T. et al., 2018: Climate change trends and impacts on California agriculture: a detailed review. Agronomy, 8(3), 25, doi:10.3390/ agronomy 8030025 .

Paul, F. and N. Mölg, 2014: Hasty retreat of glaciers in northern Patagonia from 1985 to 2011.J. Glaciol., 60(224), 1033-1043, doi:10.3189/2014JoG14J104.

Pecl, G.T. et al., 2017: Biodiversity redistribution under climate change: Impacts on ecosystems and human well-being. Science, 355(6332), eaai9214, doi:10.1126/science.aai9214.

Pedersen, S., M. Odden and H.C. Pedersen, 2017: Climate change induced molting mismatch? Mountain hare abundance reduced by duration of snow cover and predator abundance. Ecosphere, 8(3), e01722, doi:10.1002/ecs2.1722.

Pellicciotti, F. et al., 2015: Mass-balance changes of the debris-covered glaciers in the Langtang Himal, Nepal, from 1974 to 1999. J. Glaciol., 61(226), 373-386, doi:10.3189/2015jog13j237.

Penczykowski, R.M., B.M. Connolly and B.T. Barton, 2017: Winter is changing: Trophic interactions under altered snow regimes. Food Webs, 13, 80-91, doi:10.1016/j.fooweb.2017.02.006.

Peng, H. et al., 2015: Degradation characteristics of permafrost under the effect of climate warming and engineering disturbance along the Qinghai-Tibet Highway. Nat. Hazards, 75(3), 2589-2605, doi:10.1007/ s11069-014-1444-5.

Pepin, N. et al., 2015: Elevation-dependent warming in mountain regions of the world. Nat. Clim. Change, 5, 424, doi:10.1038/nclimate2563.

Pepin, N.C. and J.D. Lundquist, 2008: Temperature trends at high elevations: Patterns across the globe. Geophys. Res. Lett., 35(14), L14701, doi:10.1029/2008GL034026.

PERMOS, 2016: Permafrost in Switzerland 2010/2011 to 2013/2014 [Nötzli, J., R. Luethi and B. Staub (eds.)]. Glaciological Report Permafrost No. 12-15 of the Cryospheric Commission of the Swiss Academy of Sciences, [Available at: https://naturalsciences.ch/service/publications/82035-permafrost-inswitzerland-2010-2011-to-2013-2014]. Accessed on 08/08/2019.

Peter, H. and R. Sommaruga, 2016: Shifts in diversity and function of lake bacterial communities upon glacier retreat. ISME J., 10(7), 1545-1554, doi:10.1038/ismej.2015.245.

Petrakov, D. et al., 2016: Accelerated glacier shrinkage in the Ak-Shyirak massif, Inner Tien Shan, during 2003-2013. Sci. Total Environ., 562, 364378, doi:10.1016/j.scitotenv.2016.03.162.

Philipona, R., 2013: Greenhouse warming and solar brightening in and around the Alps. Int. J. Climatol., 33(6), 1530-1537, doi:10.1002/joc.3531.

Phillips, M. and S. Margreth, 2008: Effects of ground temperature and slope deformation on the service life of snow-supporting structures in mountain permafrost: Wisse Schijen, Randa, Swiss Alps. In: Proceedings of the 9th International Conference on Permafrost, Fairbanks, Alaska, 1990, pp. 1417-1422.
Phillips, M., E.Z. Mutter, M. Kern-Luetschg and M. Lehning, 2009: Rapid degradation of ground ice in a ventilated Talus slope: Flüela Pass, Swiss Alps. Permafrost Periglac., 20(1), 1-14, doi:10.1002/ppp.638.

Phillips, M., F. Ladner, M. Müller, U. Sambeth, J. Sorg, and P. Teysseire, 2007: Cold Regions Science and Technology, 47 (1-2 Special Issue), 32-42, doi: 10.1016/j.coldregions.2006.08.014.

Phillips, M. et al., 2017: Rock slope failure in a recently deglaciated permafrost rock wall at Piz Kesch (Eastern Swiss Alps), February 2014. Earth Surf. Process. Landf., 42(3), 426-438, doi:10.1002/esp.3992.

Pickering, C., K. Green, A.A. Barros and S. Venn, 2014: A resurvey of late-lying snowpatches reveals changes in both species and functional composition across snowmelt zones. Alpine Botany, 124(2), 93-103, doi:10.1007/ s00035-014-0140-0.

Pielmeier, C., F. Techel, C. Marty and T. Stucki, 2013: Wet snow avalanche activity in the Swiss Alps - Trend analysis for mid-winter season. In: International Snow Science Workshop Grenoble - Chamonix Mont-BlancOctober 07-11, 2013, pp. 1240-1246.

Pierce, D.W. et al., 2008: Attribution of declining Western U.S. snowpack to human effects. J. Clim., 21(23), 6425-6444, doi:10.1175/2008JCLI2405.1.

Pierson, T.C., R.J. Janda, J.C. Thouret and C.A. Borrero, 1990: Perturbation and melting of snow and ice by the 13 November 1985 eruption of Nevado-DelRuiz, Colombia, and consequent mobilization, flow and deposition of ILahars. J. Volcanol. Geoth. Res., 41(1-4), 17-66, doi:10.1016/03770273(90)90082-Q. Pintaldi, E. et al., 2017: Sustainable soil management in ski areas: Threats and challenges. Sustainability, 9, 250, doi:10.3390/ su9112150.

Plard, F. et al., 2014: Mismatch between birth date and vegetation phenology slows the demography of roe deer. PLOS Biology, 12(4), e1001828, doi:10.1371/journal.pbio.1001828.

Podolskiy, E.A., K. Nishimura, O. Abe and P.A. Chernous, 2010: Earthquake-induced snow avalanches: I. Historical case studies. J. Glaciol., 56(197), 431-446, doi:10.3189/002214310792447815.

Pogliotti, P. et al., 2015: Warming permafrost and active layer variability at Cime Bianche, Western European Alps. The Cryosphere, 9(2), 647-661, doi:10.5194/tc-9-647-2015.

Polk, M.H. et al., 2017: Exploring hydrologic connections between tropical mountain wetlands and glacier recession in Peru's Cordillera Blanca. Applied Geography, 78, 94-103, doi:10.1016/j.apgeog.2016.11.004.

Pomeroy, J.W., X. Fang and D.G. Marks, 2016: The cold rain-on-snow event of June 2013 in the Canadian Rockies - characteristics and diagnosis. Hydrol. Process., 30(17), 2899-2914, doi:10.1002/hyp.10905.

Postigo, J.C., 2014: Perception and resilience of Andean populations facing climate change. J. Ethnobiol., 34(3), 383-400, doi:10.2993/0278-077134.3.383.

Prasain, S., 2018: Climate change adaptation measure on agricultural communities of Dhye in Upper Mustang, Nepal. Clim. Change, 148(1-2), 279-291, doi:10.1007/s10584-018-2187-1.

Prasch, M., W. Mauser and M. Weber, 2013: Quantifying present and future glacier melt-water contribution to runoff in a central Himalayan river basin. The Cryosphere, 7(3), 889-904, doi:10.5194/tc-7-889-2013.

Prinz, R. et al., 2016: Climatic controls and climate proxy potential of Lewis Glacier, Mt. Kenya. The Cryosphere, 10(1), 133-148, doi:10.5194/tc-10133-2016.

Purdie, H., C. Gomez and S. Espiner, 2015: Glacier recession and the changing rockfall hazard: Implications for glacier tourism. New Zealand Geographer, 71(3), 189-202, doi:10.1111/nzg.12091.

Putkonen, J. and G. Roe, 2003: Rain-on-snow events impact soil temperatures and affect ungulate survival. Geophys. Res. Lett., 30(4), 1188, doi:10.1029/2002GL016326.

Putzer, A. and D. Festi, 2014: Nicht nur Ötzi? - Neufunde aus dem Tisental (Gem. Schnals/Prov. Bozen). Praehistorische Zeitschrift, 89(1), doi:10.1515/ pz-2014-0005. 
Qian, Y. et al., 2015: Light-absorbing particles in snow and ice: Measurement and modeling of climatic and hydrological impact. Adv. Atmos. Sci., 32(1), 64-91, doi:10.1007/s00376-014-0010-0.

Qin, J., K. Yang, S. Liang and X. Guo, 2009: The altitudinal dependence of recent rapid warming over the Tibetan Plateau. Climatic Change. 97(1), 321. doi:10.1007/s10584-009-9733-9.

Qixiang, W., M. Wang and X. Fan, 2018: Seasonal patterns of warming amplification of high-elevation stations across the globe. Int. J. Climatol., 38(8), 3466-3473, doi:10.1002/joc.5509.

Rabatel, A. et al., 2017: Toward an imminent extinction of Colombian glaciers? Geografiska Annaler. Series A, Physical Geography, 13(5), 1-21, doi:10.10 80/04353676.2017.1383015.

Rabatel, A. et al., 2013: Current state of glaciers in the tropical Andes: a multi-century perspective on glacier evolution and climate change. The Cryosphere, 7(1), 81-102, doi:10.5194/tc-7-81-2013.

Radić, V., et al., 2014: Regional and global projections of 21st century glacier mass changes in response to climate scenarios from global climate models. Clim. Dyn., 42(1-2), 37-58, doi:10.1007/s00382-013-1719-7.

Rajczak, J. and C. Schär, 2017: Projections of future precipitation extremes over europe: a multimodel assessment of climate simulations. J. Geophys. Res-Atmos., 122(20), 10-773-10-800, doi:10.1002/2017JD027176.

Raman, D., 2018: Damming and Infrastructural Development of the Indus River Basin: Strengthening the Provisions of the Indus Waters Treaty. Asian Journal of International Law, 8(2), 372-402, doi:10.1017/ S2044251317000029.

Rangwala, I., E. Sinsky and J.R. Miller, 2013: Amplified warming projections for high altitude regions of the northern hemisphere mid-latitudes from CMIP5 models. Environ. Res. Lett., 8(2), 024040, doi:10.1088/17489326/8/2/024040.

Rasmussen, M.B., 2016: Unsettling Times: Living with the Changing Horizons of the Peruvian Andes. Latin American Perspectives, 43(4), 73-86, doi:10.1177/0094582x16637867.

Rasmussen, M.B., 2019: Rewriting conservation landscapes: protected areas and glacial retreat in the high Andes. Reg. Environ. Change, 1-15, doi:10.1007/s10113-018-1376-9.

Rasul, G. and D. Molden, 2019: The global social and economic consequences of mountain cryopsheric change. Front. Environ. Sci., 7(91), doi:10.3389/ fenvs.2019.00091.

Rasul, G., B. Pasakhala, A. Mishra and S. Pant, 2019: Adaptation to mountain cryosphere change: issues and challenges. Clim. Dev. doi:10.1080/175655 29.2019.1617099.

Ravanel, L. et al., 2010: Rock falls in the Mont Blanc Massif in 2007 and 2008. Landslides, 7(4), 493-501, doi:10.1007/s10346-010-0206-z.

Ravanel, L. and P. Deline, 2011: Climate influence on rockfalls in high-Alpine steep rockwalls: The north side of the Aiguilles de Chamonix (Mont Blanc massif) since the end of the 'Little Ice Age'. The Holocene, 21(2), 357-365, doi:10.1177/0959683610374887.

Ravanel, L., P. Deline, C. Lambiel and C. Vincent, 2013: Instability of a high alpine rock ridge: the lower Arête Des Cosmiques, Mont Blanc massif, France. Geogr. Ann. A., 95(1), 51-66, doi:10.1111/geoa.12000.

Ravanel, L., F. Magnin and P. Deline, 2017: Impacts of the 2003 and 2015 summer heatwaves on permafrost-affected rock-walls in the Mont Blanc massif. Sci. Total Environ., 609, 132-143, doi:10.1016/j. scitotenv.2017.07.055.

Reggiani, P. and T.H.M. Rientjes, 2015: A reflection on the long-term water balance of the Upper Indus Basin. Hydrol. Res., 46, 446-462, doi:10.2166/ nh.2014.060.

Reyer, C.P.O. et al., 2017: Climate change impacts in Central Asia and their implications for development. Reg. Environ. Change, 17(6), 1639-1650, doi:10.1007/s10113-015-0893-z.

Reznichenko, N.V., T.R.H. Davies and D.J. Alexander, 2011: Effects of rock avalanches on glacier behaviour and moraine formation. Geomorphology, 132, 327-338, doi:10.1016/j.geomorph.2011.05.019.
RGI Consortium, 2017: Randolph Glacier Inventory-A dataset of global glacier outlines: Version 6.0: Technical Report, Global Land Ice Measurements from Space, Colorado, USA, Digital Media. doi:10.7265/N5-RGI-60.

[Available at: www.glims.org/RGl/randolph60.html].

Rhoades, R.E., X. Zapata Rios and J.A. Ochoa, 2008: Mama Cotacachi: History, local perceptions, and social impacts of climate change and glacier retreat in the Ecuadorian Andes. In: Darkening Peaks: Glacier Retreat, Science, and Society [Orlove, B., E. Wiegant and B.H. Luckman (eds.)]. University of California Press, Berkeley, pp. 216-228.

Roberts, N.J., R. McKillop, R.L. Hermanns, J.J. Clague, and T. Oppikofer, 2014: Preliminary global catalogue of displacement waves from subaerial landslides. [Sassa, K., P., Canuti, Y. Yin (eds.)]: Landslide Science for a Safer Geoenvironment. Springer International Publishing. 687-692. ISBN 9783-319-04996-0.

Rohrer, M., N. Salzmann, M. Stoffel and A.V. Kulkarni, 2013: Missing (in-situ) snow cover data hampers climate change and runoff studies in the Greater Himalayas. Sci. Total Environ., 468-469, S60-70, doi:10.1016/j. scitotenv.2013.09.056.

Rokaya, P., S. Budhathoki and K.E. Lindenschmidt, 2018: Trends in the Timing and Magnitude of Ice-Jam Floods in Canada. Sci. Rep., 8, 5834, doi:10.1038/s41598-018-24057-z.

Rosvold, J., 2016: Perennial ice and snow covered land as important ecosystems for birds and mammals. J. Biogeogr., 43, 3-12, doi:10.1111/ jbi.12609.

Round, V. et al., 2017: Surge dynamics and lake outbursts of Kyagar Glacier, Karakoram. The Cryosphere, 11(2), 723-739, doi:10.5194/tc-11-723-2017.

Rumpf, S.B., K. Huelber, N.E. Zimmermann and S. Dullinger, 2019: Elevational rear edges shifted at least as much as leading edges over the last century. Glob. Ecol. Biogeogr., 28(4), 533--543, doi:10.1111/geb.12865.

Rumpf, S.B. et al., 2018: Range dynamics of mountain plants decrease with elevation. PNAS, 115(8), 1848-1853, doi:10.1073/pnas.1713936115.

Russell, A.J. et al., 2011: A new cycle of jokulhlaups at Russell Glacier, Kangerlussuaq, West Greenland. J. Glaciol., 57(202), 238-246, doi:10.3189/002214311796405997.

Sæmundsson, p. et al., 2018: The triggering factors of the Móafellshyrna debris slide in northern Iceland: Intense precipitation, earthquake activity and thawing of mountain permafrost. Sci. Total Environ., 621, 1163-1175, doi:10.1016/j.scitotenv.2017.10.111.

Sakai, A. and K. Fujita, 2017: Contrasting glacier responses to recent climate change in high-mountain Asia. Sci. Rep., 7, 13717, doi:10.1038/s41598017-14256-5.

Sakakibara, D. and S. Sugiyama, 2014: Ice-front variations and speed changes of calving glaciers in the Southern Patagonia Icefield from 1984 to 2011. J. Geophys. Res-Earth., 119(11), 2541-2554. doi:10.1002/2014JF003148.

Sanjay, J. et al., 2017: Downscaled climate change projections for the Hindu Kush Himalayan region using CORDEX South Asia regional climate models. Adv. Clim. Change Res., 8(3), 185-198. doi:10.1016/j.accre.2017.08.003.

Sati, S.P. and V.K. Gahalaut, 2013: The fury of the floods in the north-west Himalayan region: the Kedarnath tragedy. Geomat. Nat. Haz. Risk, 4(3), 193-201, doi:10.1080/19475705.2013.827135.

Schaefli, B. et al., 2019: The role of glacier retreat for Swiss hydropower production. Renew. Energ., 132, 615-627, doi:10.1016/j. renene.2018.07.104.

Scherrer, D. and C. Körner, 2011: Topographically controlled thermal-habitat differentiation buffers alpine plant diversity against climate warming. J. Biogeogr., 38(2), 406-416, doi:10.1111/j.1365-2699.2010.02407.x.

Scherrer, S.C., P. Ceppi, M. Croci-Maspoli and C. Appenzeller, 2012: Snow-albedo feedback and Swiss spring temperature trends. Theor. Appl. Climatol., 110(4), 509-516, doi:10.1007/s00704-012-0712-0.

Schirpke, U., F. Timmermann, U. Tappeiner and E. Tasser, 2016: Cultural ecosystem services of mountain regions: Modelling the aesthetic value. Ecol. Indic., 69, 78-90, doi:10.1016/j.ecolind.2016.04.001. 
Schmidt, K.-H. and D. Morche, 2006: Sediment output and effective discharge in two small high mountain catchments in the Bavarian Alps, Germany. Geomorphology, 80(1-2), 131-145, doi:10.1016/j.geomorph.2005.09.013.

Schmidt, S. and M. Nüsser, 2017: Changes of high altitude glaciers in the Trans-Himalaya of Ladakh over the past five decades (1969-2016). Geosciences, 7(2), 27, doi:10.3390/geosciences7020027.

Schneider, D. et al., 2014: Mapping hazards from glacier lake outburst floods based on modelling of process cascades at Lake 513, Carhuaz, Peru. Advances in Geosciences, 35, 145-155, doi:10.5194/adgeo-35-145-2014.

Schneider, D., C. Huggel, W. Haeberli and R. Kaitna, 2011: Unraveling driving factors for large rock-ice avalanche mobility. Earth Surf. Process. Landf., 36(14), 1948-1966, doi:10.1002/esp.2218.

Schnorbus, M., A. Werner and K. Bennett, 2014: Impacts of climate change in three hydrologic regimes in British Columbia, Canada. Hydrol. Process., 28, 1170-1189, doi:10.1002/hyp.9661.

Schoen, E.R. et al., 2017: Future of Pacific salmon in the face of environmental change: Lessons from one of the world's remaining productive salmon regions. Fisheries, 42(10), 538-553, doi:10.1080/03632415.2017.1374251.

Scholz, K., A. Hammerle, E. Hiltbrunner and G. Wohlfahrt, 2018: Analyzing the effects of growing season length on the net ecosystem production of an alpine grassland using model-data fusion. Ecosystems, 21(5), 982-999, doi:10.1007/s10021-017-0201-5.

Schuur, E.A.G. et al., 2015: Climate change and the permafrost carbon feedback. Nature, 520, 171-179, doi:10.1038/nature14338.

Schwanghart, W. et al., 2016: Uncertainty in the Himalayan energy-water nexus: estimating regional exposure to glacial lake outburst floods. Environ. Res. Lett., 11(7), 074005, doi:10.1088/1748-9326/11/7/074005.

Schweizer, J., J.B. Jamieson and M. Schneebeli, 2003: Snow avalanche formation. Reviews of Geophysics, 41(4), 1016, doi:10.1029/2002RG000123.

Scott, D., R. Steiger, H. Dannevig and C. Aall, 2019: Climate change and the future of the Norwegian alpine ski industry. Current Issues in Tourism, doi:10.1080/13683500.2019.1608919.

Scott, D., R. Steiger, M. Rutty and Y. Fang, 2018: The changing geography of the Winter Olympic and Paralympic Games in a warmer world. Current Issues inTourism, 22(11), 1301-1311, doi:10.1080/13683500.2018.1436161.

SENASA, 2017: Áncash: Vigilancia fitosanitaria en cultivo de rosas. Servicio Nacional de Sanidad Agraria, Ministerio de Agricultura y Riego, Lima [Available at: www.senasa.gob.pe/senasacontigo/ancash-vigilanciafitosanitaria-en-cultivo-de-rosas/\#].

Seneviratne, S.I. et al., 2012: Changes in climate extremes and their impacts on the natural physical environment. [Field, C.B., V. Barros, T.F. Stocker and Q. Dahe (eds.)]. A Special Report of Working Groups I and II of the Intergovernmental Panel on Climate Change (IPCC). Cambridge University Press, Cambridge, UK, and New York, NY, USA, pp. 109-230. Cambridge University Press, Cambridge, 109-230.

Serdeczny, 0., 2019: Non-economic loss and damage and the Warsaw International Mechanism. In: Loss and Damage from Climate Change: Concepts, Methods and Policy Options [Mechler, R., L.M. Bouwer, T. Schinko, S. Surminski and J. Linnerooth-Bayer (eds.)]. Springer International Publishing, Cham, pp. 205-220.

Serrano, E. et al., 2018: Post-little ice age paraglacial processes and landforms in the high Iberian mountains: A review. Land Degrad. Dev., 29(11), 41864208, doi:10.1002/ldr.3171.

Settele, J. et al., 2014: Terrestrial and inland water systems. In: Climate change 2014: Impacts, Adaptation, and Vulnerability. Part A: Global and Sectoral Aspects. Contribution of Working Group II to the Fifth Assessment Report of the Intergovernmental Panel on Climate Change (IPCC) [Field, C.B., V.R. Barros, D.J. Dokken, K.J. Mach, M.D. Mastrandrea, T.E. Bilir, M. Chatterjee, K.L. Ebi, Y.O. Estrada, R.C. Genova, B. Girma, E.S. Kissel, A.N. Levy, S. MacCracken, P.R. Mastrandrea and L.L. White (eds.)]. Cambridge University Press, Cambridge, UK and New York, NY, USA, 271-359.
Sevestre, H. and D I. Benn, 2015: Climatic and geometric controls on the global distribution of surge-type glaciers: Implications for a unifying model of surging. J. Glaciol., 61(228), 646-662, doi:10.3189/2015JoG14J136.

Sevestre, H. et al., 2018: Tidewater Glacier Surges Initiated at the Terminus. J. Geophys. Res-Earth, 123(5), 1035-1051, doi:10.1029/2017JF004358.

Seynova, I.B. et al., 2017: Formation of water flow in lahars from active glacier-clad volcanoes. Earth's Cryosphere, 21(6), 103-111, doi:10.21782/ EC1560-7496-2017-6(103-111).

Shaheen, F.A., 2016: The art of glacier grafting: innovative water harvesting techniques in Ladakh. IWMI-Tata Water Policy Research Highlight, 8. [Available at: https://cgspace.cgiar.org/handle/10568/89600].

Shama, L.N.S. and C.T. Robinson, 2009: Microgeographic life history variation in an alpine caddisfly: plasticity in response to seasonal time constraints. Freshwater Biol., 54(1), 150-164, doi:10.1111/j.1365-2427.2008.02102.x.

Shangguan, D. et al., 2016: Characterizing the May 2015 Karayaylak Glacier surge in the eastern Pamir Plateau using remote sensing. J. Glaciol., 62(235), 944-953, doi:10.1017/jog.2016.81.

Shaoliang, Y., M. Ismail and Y. Zhaoli, 2012: Pastoral communities' perspectives on climate change and their adaptation strategies in the Hindukush-Karakoram-Himalaya. [Kreutzmann, H., (ed.)]. Springer Netherlands, Dordrecht, 307-322. doi:10.1007/978-94-007-3846-1, ISBN 978-94-007-3845-4.

Sharma, B.M. et al., 2015: Melting Himalayan glaciers contaminated by legacy atmospheric depositions are important sources of PCBs and high-molecular-weight PAHs for the Ganges floodplain during dry periods. Environ. Pollut., 206, 588-596, doi:10.1016/j.envpol.2015.08.012.

Sharma, S. et al., 2019: Widespread loss of lake ice around the Northern Hemisphere in a warming world. Nat. Clim. Change, 9(3), 227-231, doi:10.1038/s41558-018-0393-5.

Shen, Y.J. et al., 2018: Trends and variability in streamflow and snowmelt runoff timing in the southern Tianshan Mountains. J. Hydrol., 557, 173181, doi:10.1016/j.jhydrol.2017.12.035.

Shrestha, A.B. et al., 2010: Glacial lake outburst flood risk assessment of Sun Koshi basin, Nepal. Geomat. Nat. Haz. Risk, 1(2), 157-169, doi:10.1080/19475701003668968.

Shrestha, N.K., X. Du and J. Wang, 2017: Assessing climate change impacts on fresh water resources of the Athabasca River Basin, Canada. Sci. Total Environ., 601-602, 425-440, doi:10.1016/j.scitotenv.2017.05.013.

Sietz, D. and G. Feola, 2016: Resilience in the rural Andes: critical dynamics, constraints and emerging opportunities. Reg. Environ. Change, 16(8), 2163-2169, doi:10.1007/s10113-016-1053-9.

Sigl, M. et al., 2018: 19th century glacier retreat in the Alps preceded the emergence of industrial black carbon deposition on high-alpine glaciers. The Cryosphere, 12(10), 3311-3331, doi:10.5194/tc-12-3311-2018.

Sinickas, A., B. Jamieson and M.A. Maes, 2015: Snow avalanches in western Canada: investigating change in occurrence rates and implications for risk assessment and mitigation. Struct. Infrastruct. E., 12(4), 490-498, doi:10.1 080/15732479.2015.1020495.

Skarbø, K. and K. VanderMolen, 2014: Irrigation access and vulnerability to climate-induced hydrological change in the Ecuadorian Andes. Culture, Agriculture, Food and Environment, 36(1), 28-44, doi:10.1111/cuag.12027.

Skiles, S.M. et al., 2018: Radiative forcing by light-absorbing particles in snow. Nat. Clim. Change, 8(11), 965-+, doi:10.1038/s41558-018-0296-5.

Slangen, A.B.A. et al., 2017: A Review of recent updates of sea-level projections at global and regional scales. Surveys in Geophysics, 38(1), 385-406, doi:10.1007/978-3-319-56490-6_17.

Slangen, A.B.A. and R.S.W. Van De Wal, 2011: An assessment of uncertainties in using volume-area modelling for computing the twenty-first century glacier contribution to sea-level change. The Cryosphere, 5(3), doi:10.5194/ tc-5-673-2011.

Slater, A.G. and D.M. Lawrence, 2013: Diagnosing present and future permafrost from climate models. J. Clim., 26(15), 5608-5623, doi:10.1175/ jcli-d-12-00341.1. 
Slatyer, R.A., M.A. Nash and A.A. Hoffmann, 2017: Measuring the effects of reduced snow cover on Australia's alpine arthropods. Austral Ecology, 42(7), 844-857, doi:10.1111/aec.12507.

Sloat, L.L., A.N. Henderson, C. Lamanna and B.J. Enquist, 2015: The effect of the foresummer drought on carbon exchange in subalpine meadows. Ecosystems, 18(3), 533-545, doi:10.1007/s10021-015-9845-1.

Smadja, J. et al., 2015: Climate change and water resources in the Himalayas: Field study in four geographic units of the Koshi basin, Nepal. Revue de Géographie Alpine, 103(2), doi:10.4000/rga.2910.

Smith, M.W. and D.W. Riseborough, 1996: Permafrost monitoring and detection of climate change. Permafrost Periglac., 7(4), 301-309, doi:10.1002/(SICI)1099-1530(199610)7:4\&lt;301::AID-PPP231\&gt;3.0.C0;2R. Solomina, O.N. et al., 2016: Glacier fluctuations during the past 2000 years. Quaternary Sci. Rev., 149, 61-90, doi:10.1016/j.quascirev.2016.04.008.

Somers, L.D. et al., 2018: Does hillslope trenching enhance groundwater recharge and baseflow in the Peruvian Andes? Hydrol. Process., 32(3), 318-331, doi:10.1002/hyp.11423.

Somos-Valenzuela, M.A. et al., 2016: Modeling a glacial lake outburst flood process chain: the case of Lake Palcacocha and Huaraz, Peru. Hydrol. Earth Syst. Sc., 20(6), 2519-2543, doi:10.5194/hess-20-2519-2016.

Song, C., B. Huang, L. Ke and K.S. Richards, 2014: Remote sensing of alpine lake water environment changes on the Tibetan Plateau and surroundings: A review. ISPRS J. Photogram., 92, 26-37, doi:10.1016/j. isprsjprs.2014.03.001.

Sørensen, L.S. et al., 2017: The effect of signal leakage and glacial isostatic rebound on GRACE-derived ice mass changes in Iceland. Geophys. J. Int., 209, 226-233, doi:10.1093/gji/ggx008.

Sorg, A. et al., 2015: Contrasting responses of Central Asian rock glaciers to global warming. Sci. Rep., 5, 8228, doi:10.1038/srep08228.

Soruco, A. et al., 2015: Contribution of glacier runoff to water resources of La Paz city, Bolivia (16º S). Ann. Glaciol., 56(70), 147-154, doi:10.3189/2015AoG70A001.

Spandre, P. et al., 2019a: Climate controls on snow reliability in French Alps ski resorts. Sci. Rep., 9, 8043, doi:10.1038/s41598-019-44068-8.

Spandre, P. et al., 2019b: Winter tourism under climate change in the Pyrenees and the French Alps: relevance of snowmaking as a technical adaptation. The Cryosphere, 13(4), 1325-1347, doi:10.5194/tc-13-1325-2019.

Stäubli, A. et al., 2018: Analysis of Weather - and Climate-Related Disasters in Mountain Regions Using Different Disaster Databases. In: Climate Change, Extreme Events and Disaster Risk Reduction. Sustainable Development Goals Series [Mal S., Singh R. and C. Huggel (eds.)]. Springer International Publishing, Cham,17-41.

Stearns, L.A. et al., 2015: Glaciological and marine geological controls on terminus dynamics of Hubbard Glacier, southeast Alaska. J. Geophys. Res-Earth, 120(6), 1065-1081, doi:10.1002/2014jf003341.

Steiger, R. et al., 2017: A critical review of climate change risk for ski tourism. Current Issues in Tourism, 22(11), 1343-1379, doi:10.1080/13683500.20 17.1410110.

Steinbauer, M.J. et al., 2016: Topography-driven isolation, speciation and a global increase of endemism with elevation. Global Ecol. Biogeogr., 25, 1097-1107, doi:10.1111/geb.12469.

Steinbauer, M.J. et al., 2018: Accelerated increase in plant species richness on mountain summits is linked to warming. Nature, 556(7700), 231-234, doi:10.1038/s41586-018-0005-6.

Steiner, J.F., P.D.A. Kraaijenbrink, S.G. Jiduc and W.W. Immerzeel, 2018: Brief communication: The Khurdopin glacier surge revisited - Extreme flow velocities and formation of a dammed lake in 2017. The Cryosphere, 12(1), 95-101, doi:10.5194/tc-12-95-2018.

Steinkogler, W., B. Sovilla and M. Lehning, 2014: Influence of snow cover properties on avalanche dynamics. Cold Reg. Sci. Technol., 97, 121-131, doi:10.1016/j.coldregions.2013.10.002.

Stenseth, N.C. et al., 2003: Review article. Studying climate effects on ecology through the use of climate indices: the North Atlantic Oscillation, El Niño
Southern Oscillation and beyond. Proc. Royal Soc. B., 270 (1529), 20872096, doi:10.1098/rspb.2003.2415.

Stewart, E.J. et al., 2016: Implications of climate change for glacier tourism. Tourism Geographies, 18(4), 377-398, doi:10.1080/14616688.2016.1198 416.

Stocker, T.F. et al., 2013: IPCC Technical Summary AR5. In: Climate Change 2013: The Physical Science Basis. Contribution of Working Group I to the Fifth Assessment Report of the Intergovernmental Panel on Climate Change [Stocker, T.F., D. Qin, G.-K. Plattner, M. Tignor, S.K. Allen, J. Boschung, A. Nauels, Y. Xia, V. Bex and P.M. Midgley (eds.)]. Cambridge University Press, Cambridge, United Kingdom and New York, NY, USA, 1535 pp.

Stoffel, M. and C. Graf, 2015: Debris-flow activity from high-elevation, periglacial environments. [Huggel, C., M. Carey, J.J. Clague and A. Kääb (eds.)]. Cambridge University Press, Cambridge, 295-314, ISBN 978-1-10706584-0.

Stucker, D., J. Kazbekov, M. Yakubov and K. Wegerich, 2012: Climate change in a small transboundary tributary of the Syr Darya Calls for effective cooperation and adaptation. Mt. Res. Dev., 32(3), 275-285, doi:10.1659/ MRD-JOURNAL-D-11-00127.1.

Sturm, M., M.A. Goldstein and C. Parr, 2017: Water and life from snow: A trillion dollar science question. Water Resour. Res., 53(5), 3534-3544, doi:10.1002/2017WR020840.

Suding, K.N. et al., 2015: Vegetation change at high elevation: scale dependence and interactive effects on Niwot Ridge. Plant Ecol. Divers., 8(5-6), 713-725, doi:10.1080/17550874.2015.1010189.

Sujakhu, N.M. et al., 2016: Farmers' perceptions of and adaptations to changing climate in the Melamchi Valley of Nepal. Mt. Res. Dev., 36(1), 15-30, doi:10.1659/MRD-JOURNAL-D-15-00032.1.

Sultaire, S.M. et al., 2016: Climate change surpasses land-use change in the contracting range boundary of a winter-adapted mammal. Proc. R. Soc. B., 283(1831), doi:10.1098/rspb.2016.0899.

Sultana, R. and M. Choi, 2018: Sensitivity of streamflow response in the snow-dominated Sierra Nevada Watershed using projected CMIP5 data. J. Hydrol. Eng., 23(8), 05018015, doi:10.1061/(ASCE)HE.19435584.0001640.

Sun, J. et al., 2018a: Linkages of the dynamics of glaciers and lakes with the climate elements over the Tibetan Plateau. Earth-Sci. Rev., 185, 308-324, doi:10.1016/j.earscirev.2018.06.012.

Sun, X. et al., 2017: The role of melting alpine glaciers in mercury export and transport: An intensive sampling campaign in the Qugaqie Basin, inland Tibetan Plateau. Environ. Pollut., 220, 936-945, doi:10.1016/j. envpol.2016.10.079.

Sun, X. et al., 2018b: Mercury speciation and distribution in a glacierized mountain environment and their relevance to environmental risks in the inland Tibetan Plateau. Sci. Total Environ., 631-632, 270-278, doi:10.1016/j.scitotenv.2018.03.012.

Surfleet, C.G. and D. Tullos, 2013: Variability in effect of climate change on rain-on-snow peak flow events in a temperate climate. J. Hydrol., 479, 24-34, doi:10.1016/J.JHYDROL.2012.11.021.

Suzuki-Parker, A., Y. Miura, H. Kusaka and M. Kureha, 2018: Assessing the Sustainability of Ski Fields in Southern Japan under Global Warming. Advances in Meteorology, 2018(8529748), 1-10, doi:10.1155/2018/8529748.

Swindles, G.T. et al., 2018: Climatic control on Icelandic volcanic activity during the mid-Holocene. Geology, 46(1), 47-50, doi:10.1130/G39633.1.

Taylor, K.E., R.J. Stouffer and G.A. Meehl, 2012: An overview of CMIP5 and the experiment design. Bull. Am. Meterol. Soc., 93, 485-498, doi:10.1175/ BAMS-D-11-00094.1.

Teich, M. et al., 2012: Snow and weather conditions associated with avalanche releases in forests: Rare situations with decreasing trends during the last 41 years. Cold Reg. Sci. Technol., 83-84, 77-88, doi:10.1016/j. coldregions.2012.06.007. 
Temme, A.J.A.M., 2015: Using climber's guidebooks to assess rock fall patterns over large spatial and decadal temporal scales: An example from the Swiss Alps. Geogr. Ann. A., 97(4), 793-807, doi:10.1111/geoa.12116.

Terzago, S., J.v. Hardenberg, E. Palazzi and P. Antonello, 2014: Snowpack changes in the Hindu Kush-Karakoram-Himalaya from CMIP5 Global Climate Models. J. Hydrometeorol., 15(6), 2293-2313, doi:10.1175/ JHM-D-13-0196.1.

Theobald, E.J. et al., 2015: Global change and local solutions: Tapping the unrealized potential of citizen science for biodiversity research. Biol. Conserv., 181, 236-244, doi:10.1016/j.biocon.2014.10.021.

Thibert, E. et al., 2018: Causes of glacier melt extremes in the Alps since 1949. Geophys. Res. Lett., 45(2), 817-825, doi:10.1002/2017GL076333.

Thies, H. et al., 2013: Evidence of rock glacier melt impacts on water chemistry and diatoms in high mountain streams. Cold Reg. Sci. Technol., 96, 77-85, doi:10.1016/j.coldregions.2013.06.006.

Thompson, L.G. et al., 2017: Impacts of Recent Warming and the 2015/2016 El Niño on Tropical Peruvian Ice Fields. J. Geophys. Res-Earth, 122(23), 12,688-12,701, doi:10.1002/2017JD026592.

Tiwari, P.C. and B. Joshi, 2015: Climate Change and Rural Out-migration in Himalaya. Change and Adaptation in Socio-Ecological Systems, 2, 8-25, doi:10.1515/cass-2015-0002.

Torgoev, I. and B. Omorov, 2014: Mass movement in the waste dump of high-altitude Kumtor Goldmine (Kyrgyzstan). [Sassa, K., Canuti, P., Yin, Y. (Eds.)]: Landslide Science for a Safer Geoenvironment. Springer International Publishing. 517-521. ISBN 978-3-319-04996-0.

Toropov, P.A., M.A. Aleshina and A.M. Grachev, 2019: Large-scale climatic factors driving glacier recession in the Greater Caucasus, 20th-21st century. Int. J. Climatol., 39 (12), 4703-4720, doi:10.1002/joc.6101.

Trujillo, E. et al., 2012: Elevation-dependent influence of snow accumulation on forest greening. Nat. Geosci., 5, 705-709, doi:10.1038/ngeo1571.

Tschakert, P. et al., 2019: One thousand ways to experience loss: A systematic analysis of climate-related intangible harm from around the world. Glob. Environ. Change, 55, 58-72, doi:10.1016/j.gloenvcha.2018.11.006.

Tudoroiu, M. et al., 2016: Negative elevation-dependent warming trend in the Eastern Alps. Environ. Res. Lett., 11(4), doi:10.1088/17489326/11/4/044021.

UN, 2015: Transforming governance for the 2030 agenda for sustainable development. UN, New York, NY, [Available at: https:// sustainabledevelopment.un.org/content/documents/21252030\%20 Agenda\%20for\%20Sustainable\%20Development\%20web.pdf].

UNESCO, 1972: Convention Concerning the Protection of the World Cultural and Naturral Heritage. Adopted by the General Conference at its seventeenth session Paris, 16 november 1972. United Nations Educational, Scientific, and Cultural Organisation (UNESCO), Paris, [Available at: https://whc.unesco.org/archive/convention-en.pdf]. Accessed 08/08/2019.

UNESCO, 2012: Operational Guidelines for the Implementation of the World Heritage Convention. United Nations Educational, Scientific And Cultural Organisation (UNESCO), Paris. [Available at: http://whc.unesco.org/en/ guidelines]. Accessed 08/08/2019.

UNFCCC, 2015: Paris Agreement. United Nations. Climate Change Secretariat, UNEP's Information Unit for Conventions (IUC), Bonn, Germany, 30pp. [Available at: http://unfccc.int/files/essential_background/convention/ application/pdf/english_paris_agreement.pdf].

UNFCCC Secretariat, 2014: Subsidiary body for scientific and technological advice. Forty-first session, Lima 1-6 December 2014. Report of the executive committee of the Warsaw international mechanism for loss and damage associated with climate change impacts. UNFCCC, Lima, [Available at: https://unfccc.int/resource/docs/2014/sb/eng/04.pdf]. Accessed 08/08/2019.

UNHRC, 2018: Resolution adopted by the General Assembly on 26 September 2018: United Nations Declaration on the Rights of Peasants and Other People Working in Rural Areas. UNHRC 39th Assembly General [Available at: https://undocs.org/A/HRC/39/L.16].
UNISDR, 2015: Sendai Framework for Disaster Risk Reduction 2015-2030, Geneva, The United Nations Office for Disaster Risk Reduction. [Available at: www.unisdr.org/files/43291_sendaiframeworkfordrren.pdf].

Uniyal, A., 2013: Lessons from Kedarnath tragedy of Uttarakhand Himalaya, India. Current Science, 105(11), 1472-1474.

Vaidya, R.A. et al., 2019: Disaster Risk Reduction and Building Resilience in the Hindu Kush Himalaya. In: The Hindu Kush Himalaya Assessment: Mountains, Climate Change, Sustainability and People [Wester, P., A. Mishra, A. Mukherji and A.B. Shrestha (eds.)]. Springer International Publishing, Cham, pp. 389-419. ISBN 9783319922874.

Valdés-Pineda, R. et al., 2014: Water governance in Chile: Availability, management and climate change. J. Hydrol., 519, 2538-2567, doi:10.1016/j.jhydrol.2014.04.016.

Vallance, J.W., 2005: Volcanic debris flows. [M. Jakob and O. Hungr (eds.)], Debris-flow Hazards and Related Phenomena. Springer Berlin Heidelberg, Berlin, Heidelberg. 247-274. ISBN 978-3-540-27129-1, doi:10.1007/3540-27129-5 10.

van der Geest, K. and M. Schindler, 2016: Brief communication: Loss and damage from a catastrophic landslide in Nepal. Nat. Hazard. Earth Sys., 16(11), 2347-2350, doi:10.5194/nhess-16-2347-2016.

van der Woerd, J. et al., 2004: Giant, M8 earthquake-triggered ice avalanches in the eastern Kunlun Shan, northern Tibet: Characteristics, nature and dynamics. Bull. Geol. Soc. Am., 116(3-4), 394-406, doi:10.1130/B25317.1.

Vanat, L., 2018: 2018 International Report on Snow \& Mountain Tourism. Overview of the key industry figures for ski resorts. [Available at: https://vanat.ch/RM-world-report-2018.pdf].

Veh, G., O. Korup, S. Roessner and A. Walz, 2018: Detecting Himalayan glacial lake outburst floods from Landsat time series. Remote Sens. Environ., 207, 84-97. doi:10.1016/j.rse.2017.12.025.

Veh, G. et al., 2019: Unchanged frequency of moraine-dammed glacial lake outburst floods in the Himalaya. Nat. Clim. Change, 9(5), 379-383, doi:10.1038/s41558-019-0437-5.

Vergara, W. et al., 2007: Economic impacts of rapid glacier retreat in the Andes. Eos, Trans. AGU, 88(25), 261-264, doi:10.1029/2007E0250001.

Vermilyea, A.W. et al., 2017: Continuous proxy measurements reveal large mercury fluxes from glacial and forested watersheds in Alaska. Sci. Total Environ., 599-600, 145-155, doi:10.1016/j.scitotenv.2017.03.297.

Viazzo, P.P., 1989: Upland communities: Environment, populations and social structure in the Alps since the sixteenth century. Cambridge University Press, Cambridge. ISBN: 9780521034166.

Vigano, G. et al., 2016: Effects of Future Climate Change on a River Habitat in an Italian Alpine Catchment. J. Hydrol. Eng., 21(2), doi:10.1061/(ASCE) HE.1943-5584.0001293.

Vince, G., 2009: Profile: Chewang Norphel. Glacier man. American Association for the Advancement of Science, 326, 659-661, doi:10.1126/ science.326_659.

Viviroli, D. et al., 2011: Climate change and mountain water resources: overview and recommendations for research, management and policy. Hydrol. Earth Syst. Sc., 15(2), 471-504, doi:10.5194/hess-15-471-2011.

Vors, L.S. and M.S. Boyce, 2009: Global declines of caribou and reindeer. Glob. Change Biol, 15(11), 2626-2633, doi:10.1111/j.1365-2486.2009.01974.x.

Vuille, M., 2013: Climate change and water resources in the tropical Andes. Inter-American Development Bank Technical Note 515. [Available at: https://publications.iadb.org/handle/11319/5827].

Vuille, M. et al., 2018: Rapid decline of snow and ice in the tropical Andes Impacts, uncertainties and challenges ahead. Earth-Sci. Rev., 176, 195213, doi:10.1016/j.earscirev.2017.09.019.

Waechter, A., L. Copland and E. Herdes, 2015: Modern glacier velocities across the Icefield Ranges, St Elias Mountains, and variability at selected glaciers from 1959 to 2012. J. Glaciol., 61(228), 624-634, doi:10.3189/2015JoG14J147. 
Walter, D., 2017: Percepciones tradicionales del cambio climático en comunidades altoandinas en la Cordillera Blanca, Ancash. Revista de Glaciares y Ecosistemas de Montaña, 3, 9-24.

Wang, L. et al., 2015: Glacier changes in the Sikeshu River basin, Tienshan Mountains. Quaternary International, 358, 153-159, doi:10.1016/j. quaint.2014.12.028.

Wang, Q., X. Fan and M. Wang, 2016a: Evidence of high-elevation amplification versus Arctic amplification. Sci. Rep., 6 (19219), doi:10.1038/srep19219.

Wang, S., Y. He and X. Song, 2010: Impacts of climate warming on Alpine glacier tourism and adaptive measures: A case study of Baishui Glacier No. 1 in Yulong Snow Mountain, Southwestern China. J. Earth Sci., 21(2), 166-178, doi:10.1007/s12583-010-0015-2.

Wang, S. et al., 2017: Complex responses of spring alpine vegetation phenology to snow cover dynamics over the Tibetan Plateau, China. Sci. Total Environ., 593-594, 449-461, doi:10.1016/j.scitotenv.2017.03.187.

Wang, $X$. et al., 2018: Snow cover phenology affects alpine vegetation growth dynamics on the Tibetan Plateau: Satellite observed evidence, impacts of different biomes, and climate drivers. Agr. Forest Meterol., 256-257, 6174, doi:10.1016/j.agrformet.2018.03.004.

Wang, X. et al., 2016b: The role of permafrost and soil water in distribution of alpine grassland and its NDVI dynamics on the Qinghai-Tibetan Plateau. Glob. Planet. Change, 147, 40-53, doi:10.1016/J.GLOPLACHA.2016.10.014.

Wangchuk, K. and J. Wangdi, 2018: Signs of climate warming through the eyes of yak herders in northern Bhutan. Mt. Res. Dev., 38(1), 45-52, doi:10.1659/MRD-JOURNAL-D-17-00094.1.

Warren, F J. and D.S. Lemmen, 2014: Canada in a Changing Climate: Sector Perspectives on Impacts and Adaptation. Government of Canada, Ottawa, ON, 286pp. ISBN: 978-1-100-24142-5. [Available at: www.weadapt.org/ sites/weadapt.org/files/2017/february/canadasectorperspectivesfullreport_eng_0.pdf\#page=70].

Watson, C.S. and O. King, 2018: Everest's thinning glaciers: implications for tourism and mountaineering. Geology Today, doi:10.1111/gto.12215.

Wegner, S.A., 2014: Lo que el agua se llevó: Consecuencias y lecciones del aluvión de Huaraz de 1941. Notas Técnicas sobre Cambio Climático 7, Ministerio de Ambiente, Lima, [Available at: https://archive.org/details/ NotaTecnica7/page/n1].

Wei, M., N. Fujun, A. Satoshi and A. Dewu, 2006: Slope instability phenomena in permafrost regions of Qinghai-Tibet Plateau, China. Landslides, 3(3), 260-264, doi:10.1007/s10346-006-0045-0.

Welling, J., R. Ólafsdóttir, P. Árnason and S. Guð̌mundsson, 2019: Participatory Planning Under Scenarios of Glacier Retreat and Tourism Growth in Southeast Iceland. Mt. Res. Dev., 39 (2), D1-D13, doi:10.1659/ MRD-JOURNAL-D-18-00090.1

Wester, P., A. Mishra, A. Mukherji and A.B. Shrestha (eds.), 2019: The Hindu Kush Himalaya Assessment - Mountains, Climate Change, Sustainability and People. Springer, 627 pp. ISBN 9783319922881.

Westerling, A.L., 2016: Increasing western US forest wildfire activity: sensitivity to changes in the timing of spring. Philos. Trans. R. Soc. London (Biol)., 371(1696), 20150178, doi:10.1098/rstb.2015.0178.

Westermann, S. et al., 2015: A ground temperature map of the North Atlantic permafrost region based on remote sensing and reanalysis data. The Cryosphere, 9(3), 1303-1319, doi:10.5194/tc-9-1303-2015.

White, K.S., D.P. Gregovich and T. Levi, 2017: Projecting the future of an alpine ungulate under climate change scenarios. Glob. Change Biol, 24(3), 113601149, doi:10.1111/gcb.13919.

Wikstrom Jones, K. et al., 2018: Community Snow Observations (CSO): A citizen science campaign to validate snow remote sensing products and hydrological models. In: International Snow Science Workshop, Innsbruck, Austria, pp. 420-424.

Williams, C.M., H.A.L. Henry and B.J. Sinclair, 2015: Cold truths: how winter drives responses of terrestrial organisms to climate change. Biol. Rev., 90(1), 214-235, doi:10.1111/brv.12105.
Williamson, C.J. et al., 2019: Glacier Algae: A Dark Past and a Darker Future. Front. Microbiol. 10, 519, doi:10.3389/fmicb.2019.00524.

Willis, M.J. et al., 2018: Massive destabilization of an Arctic ice cap. Earth Planet Sc. Lett., 502, 146-155, doi:10.1016/j.epsl.2018.08.049.

Winkler, D.E. et al., 2018: Snowmelt timing regulates community composition, phenology, and physiological performance of alpine plants. Front. Plant. Sci. 9, 1140, doi:10.3389/fpls.2018.01140.

Winkler, D.E., K.J. Chapin and L.M. Kueppers, 2016: Soil moisture mediates alpine life form and community productivity responses to warming. Ecology, 97(6), 1553-1563, doi:10.1890/15-1197.1.

Winski, D. et al., 2017: Industrial-age doubling of snow accumulation in the Alaska Range linked to tropical ocean warming. Sci. Rep., 7, 17869, doi:10.1038/s41598-017-18022-5.

Winter, K.J.P.M., S. Kotlarski, S.C. Scherrer and C. Schär, 2017: The Alpine snow-albedo feedback in regional climate models. Clim. Dyn., 48(3-4), 1109-1124, doi:10.1007/s00382-016-3130-7.

Wirz, V., M. Geertsema, S. Gruber and R.S. Purves, 2016: Temporal variability of diverse mountain permafrost slope movements derived from multi-year daily GPS data, Mattertal, Switzerland. Landslides, 13 (1), 67-83, doi:10.1007/s10346-014-0544-3.

Wobus, C. et al., 2017: Projected climate change impacts on skiing and snowmobiling: A case study of the United States. Glob. Environ. Change, 45, 1-14 doi:10.1016/j.gloenvcha.2017.04.006.

Wouters, B., A.S. Gardner and G. Moholdt, 2019: Global glacier mass loss during the GRACE satellite mission (2002-2016). Front. Earth Sci., 7(96), doi:10.3389/feart.2019.00096.

Wrathall, D.J. et al., 2014: Migration Amidst Climate Rigidity Traps: Resource Politics and Social-Ecological Possibilism in Honduras and Peru. Ann. Am. Assoc. Geogr., 104(2), 292-304, doi:10.1080/00045608.2013.873326.

Wu, Q., Y. Hou, H. Yun and Y. Liu, 2015: Changes in active-layer thickness and near-surface permafrost between 2002 and 2012 in alpine ecosystems, Qinghai-Xizang (Tibet) Plateau, China. Glob. Planet. Change, 124, 149155, doi:10.1016/j.gloplacha.2014.09.002.

$\mathrm{Wu}, \mathrm{X}$. et al., 2018: Uneven winter snow influence on tree growth across temperate China. Glob. Change Biol, 25(1), 144-154, doi:10.1111/ gcb.14464.

Würzer, S., T. Jonas, N. Wever and M. Lehning, 2016: Influence of Initial Snowpack Properties on Runoff Formation during Rain-on-Snow Events. J. Hydrometeorol., 17(6), 1801-1815, doi:10.1175/JHM-D-15-0181.1.

Wymann von Dach, S. et al., 2017: Safer lives and livelihoods in mountains: Making the Sendai framework for disaster risk reduction work for sustainable mountain development. Centre for Development and Environment (CDE), University of Bern, with Bern Open Publishing (BOP), Bern, Switzerland, 82 pp.

Wymann von Dach, S. et al., 2018 Leaving no one in mountains behind: Localizing the SDGs for resilience of mountain people and ecosystems. Mountain Research Initiative and Centre for Development and Environment, Bern, Switzerland, [Available at: https://boris.unibe.ch/id/ eprint/120130]. Accessed 08/08/2019.

Xenarios, S. et al., 2018: Climate change and adaptation of mountain societies in Central Asia: uncertainties, knowledge gaps, and data constraints. Reg. Environ. Change, 31(3-4), 1113, doi:10.1007/s10113-018-1384-9.

Xiao, C.-D., S.-J. Wang and D.H. Qin, 2015: A preliminary study of cryosphere service function and value evaluation. Adv. Clim. Change Res., 6(3-4), 181-187, doi:10.1016/j.accre.2015.11.004.

Xie, J. et al., 2018: Relative influence of timing and accumulation of snow on alpine land surface phenology. J. Geophys. Res.-Biogeosci., 123(2), 561576, doi:10.1002/2017JG004099.

Xu, Y., V. Ramanathan and W.M. Washington, 2016: Observed high-altitude warming and snow cover retreat over Tibet and the Himalayas enhanced by black carbon aerosols. Atmos. Chem. Phys., 16(3), 1303-1315, doi:10.5194/acp-16-1303-2016. 
Yager, K., 2015: Saiellite Imagery and community perceptions of climate change impacts and landscape change. [Barnes, J. and M. Dove (eds.)], Climate Cultures: Anthropological Perspectives on Climate Change. New Haven, Yale University Press, 146-168.

Yang, K. et al., 2014a: Recent climate changes over the Tibetan Plateau and their impacts on energy and water cycle: A review. Glob. Planet. Change, 112, 79-91, doi:10.1016/J.GLOPLACHA.2013.12.001.

Yang, M. et al., 2010a: Permafrost degradation and its environmental effects on the Tibetan Plateau: A review of recent research. Earth-Sci. Rev., 103 (1-2), 31-44, doi:10.1016/j.earscirev.2010.07.002.

Yang, Y. et al., 2018: Permafrost and drought regulate vulnerability of Tibetan Plateau grasslands to warming. Ecosphere, 9(5), e02233, doi:10.1002/ ecs2.2233.

Yang, Y.-C.E. et al., 2014b: Water governance and adaptation to climate change in the Indus River Basin. J. Hydrol., 519, 2527-2537, doi:10.1016/j. jhydrol.2014.08.055.

Yang, Z.-p. et al., 2010b: Effects of permafrost degradation on ecosystems. Acta Ecologica Sinica, 30(1), 33-39, doi:10.1016/j.chnaes.2009.12.006.

Yao, X. et al., 2016: Spatial-temporal variations of lake ice phenology in the Hoh Xil region from 2000 to 2011. J. Geogr. Sci. , 26(1), 70-82, doi:10.1007/ s11442-016-1255-6.

Yasuda, T. and M. Furuya, 2015: Dynamics of surge-type glaciers in West Kunlun Shan, Northwestern Tibet. J. Geophys. Res-Earth, 120(11), 23932405, doi:10.1002/2015JF003511.

Yasunari, T.J., R.D. Koster, W.K.M. Lau and K.-M. Kim, 2015: Impact of snow darkening via dust, black carbon, and organic carbon on boreal spring climate in the Earth system. J. Geophys. Res-Earth, 120(11), 5485-5503, doi:10.1002/2014JD022977.

Ye, H., D. Yang and D. Robinson, 2008: Winter rain on snow and its association with air temperature in northern Eurasia. Hydrol. Process., 22(15), 27282736, doi:10.1002/hyp.7094.

You, J. et al., 2018: Response to climate change of montane herbaceous plants in the genus Rhodiola predicted by ecological niche modelling. Sci. Rep., 8, 1-12, doi:10.1038/s41598-018-24360-9.

You, Q. et al., 2010: Climate warming and associated changes in atmospheric circulation in the eastern and central Tibetan Plateau from a homogenized dataset. Glob. Planet. Change, 72, 11-24, doi:10.1016/j. gloplacha.2010.04.003.

You, Q. et al., 2013: Decadal variation of surface solar radiation in the Tibetan Plateau from observations, reanalysis and model simulations. Clim. Dyn., 40(7-8), 2073-2086, doi:10.1007/s00382-012-1383-3.

Young, E.F. et al., 2018: Stepping stones to isolation: Impacts of a changing climate on the connectivity of fragmented fish populations. Evol. Appl., 11(6), 978--994, doi:10.1111/eva.12613.

Young, G. et al., 2010: Vulnerability and adaptation in a dryland community of the Elqui Valley, Chile. Clim. Change, 98(1-2), 245-276, doi:10.1007/ s10584-009-9665-4.

Yu, F., J. Qi, X. Yao and Y. Liu, 2013: In-situ monitoring of settlement at different layers under embankments in permafrost regions on the Qinghai-Tibet Plateau. Eng. Geol., 160, 44-53, doi:10.1016/j.enggeo.2013.04.002.

Yu, W., F. Han, W. Liu and S.A. Harris, 2016: Geohazards and thermal regime analysis of oil pipeline along the Qinghai-Tibet Plateau Engineering Corridor. Nat. Hazards, 83(1), 193-209, doi:10.1007/s11069-016-2308-y.

Yu, Y. et al., 2019: Climate change, water resources and sustainable development in the arid and semi-arid lands of Central Asia in the past 30 years. J. Arid Land, 11(1), 1-14, doi:10.1007/s40333-018-0073-3.

Yucel, I., A. Güventürk and O.L. Sen, 2015: Climate change impacts on snowmelt runoff for mountainous transboundary basins in eastern Turkey. Int. J. Climatol., 35(2), 215-228, doi:10.1002/joc.3974.

Zaharescu, D.G. et al., 2016: Climate change enhances the mobilisation of naturally occurring metals in high altitude environments. Sci. Total Environ., 560-561, 73-81, doi:10.1016/j.scitotenv.2016.04.002.
Zazulie, N., 2016: Estudio de los distintos factores que afectaron la evolución de los glaciares en los Andes Centrales del Sur y sus proyecciones ante posibles escenarios de cambio climático. Tesis Doctoral, Facultad de Ciencias Exactas y Naturales. Universidad de Buenos Aires. [Available at: http://hdl.handle.net/20.500.12110/tesis_n5842_Zazulie].

Zazulie, N., M. Rusticucci and G.B. Raga, 2018: Regional climate of the Subtropical Central Andes using high-resolution CMIP5 models. Part II: future projections for the twenty-first century. Clim. Dyn., 51(7-8), 29132925, doi:10.1007/s00382-017-4056-4.

Zdanowicz, C. et al., 2013: Accumulation, storage and release of atmospheric mercury in a glaciated Arctic catchment, Baffin Island, Canada. Geochimica et Cosmochimica Acta, 107, 316-335, doi:10.1016/j.gca.2012.11.028.

Zekollari, H., M. Huss and D. Farinotti, 2019: Modelling the future evolution of glaciers in the European Alps under the EURO-CORDEX RCM ensemble. The Cryosphere, 13(4), 1125-1146, doi:10.5194/tc-13-1125-2019.

Zemp, M. et al., 2015: Historically unprecedented global glacier decline in the early 21st century. J. Glaciol., 61(228), 745-762, doi:10.3189/2015JoG15J017.

Zemp, M. et al., 2019: Global glacier mass changes and their contributions to sea-level rise from 1961 to 2016 Nature, 568(7752), 382-386 doi:10.1038/ s41586-019-1071-0.

Zeng, Z. et al., 2015: Regional air pollution brightening reverses the greenhouse gases induced warming-elevation relationship. Geophys. Res. Lett., 42(11), 4563-4572, doi:10.1002/2015GL064410.

Zhang, G. et al., 2014: Estimating surface temperature changes of lakes in the Tibetan Plateau using MODIS LST data. J. Geophys. Res., 119(14), 85528567, doi:10.1002/2014JD021615.

Zhang, G. et al., 2015: An inventory of glacial lakes in the Third Pole region and their changes in response to global warming. Glob. Planet. Change, 131, 148-157, doi:10.1016/j.gloplacha.2015.05.013.

Zhang, H.-X. and M.-L. Zhang, 2017: Spatial patterns of species diversity and phylogenetic structure of plant communities in the Tianshan Mountains, arid Central Asia. Front. Plant Sci., 8, 2134, doi:10.3389/fpls.2017.02134.

Zhang, Q. et al., 2012: Mercury distribution and deposition in glacier snow over western China. Environ. Sci. Technol., 46(10), 5404-5413. doi:10.1021/es300166x.

Zhang, Y. et al., 2017: Light-absorbing impurities enhance glacier albedo reduction in the southeastern Tibetan plateau. J. Geophys. Res-Atmos, 122(13), 6915-6933, doi:10.1002/2016JD026397.

Zhang, Y. et al., 2018: Black carbon and mineral dust in snow cover on the Tibetan Plateau. The Cryosphere, 12(2), 413-431, doi:10.5194/tc-12-4132018.

Zhao, L., Q. Wu, S. Marchenko and N. Sharkhuu, 2010: Thermal state of permafrost and active layer in central Asia during the international polar year. Permafrost Periglac., 21(2), 198-207, doi:10.1002/ppp.688.

Zhou, Y., Z. Li and J. Li, 2017: Slight glacier mass loss in the Karakoram region during the 1970 s to 2000 revealed by KH-9 images and SRTM DEM.J. Glaciol., 63(238), 331-342, doi:10.1017/jog.2016.142.

Ziegler, A.D. et al., 2014: Pilgrims, progress, and the political economy of disaster preparedness - the example of the 2013 Uttarakhand flood and Kedarnath disaster. Hydrol. Process., 28(24), 5985-5990, doi:10.1002/ hyp.10349.

Zimmer, A. et al., 2018: Time lag between glacial retreat and upward migration alters tropical alpine communities. Perspect. Plant Ecol. Evol. Syst., 30, 89102, doi:10.1016/..ppees.2017.05.003.

Zimmermann, M. and W. Haeberli, 1992: Climatic change and debris flow activity in high-mountain areas - a case study in the Swiss Alps. Catena Supplement, 22, 59-72.

Zimov, S.A., E.A.G. Schuur and F.S. Chapin, 2006: Permafrost and the global carbon budget. Science, 312(5780), 1612-1613, doi:10.1126/ science. 1128908 . 
Zimova, M., L.S. Mills and J.J. Nowak, 2016: High fitness costs of climate change-induced camouflage mismatch. Ecol. Letters, 19(3), 299-307, doi:10.1111/ele.12568.

Zimova, M. and Hackländer, K. and Good, J.M. and Melo-Ferreira, J. and Alves, P.C. and Mills, L.S., 2018. Function and underlying mechanisms of seasonal colour moulting in mammals and birds: what keeps them changing in a warming world?. Biol. Rev., 93(3): 1478-1498. doi:10.1111/brv.12405.

Zou, D. et al., 2017: A new map of permafrost distribution on the Tibetan Plateau. The Cryosphere, 11(6), 2527-2542, doi:10.5194/tc-11-2527-2017.

Zuckerberg, B. and J.N. Pauli, 2018: Conserving and managing the subnivium. Conserv. Biol., 32(4), 774-781, doi:10.1111/cobi.13091.

Pórhallsdóttir, G. and R. Ólafsson, 2017: A method to analyse seasonality in the distribution of tourists in Iceland. J. Outdoor Recreat., 19, 17-24, doi:10.1016/j.jort.2017.05.001. 


\section{Appendix 2.A: Additional Information on Global and Regional Glacier Mass Change Estimates for 2006-2015}

Two global-scale estimates of recent glacier mass changes have been published since AR5 (Wouters et al., 2019; Zemp et al., 2019) that include area-averaged estimates for large-scale glacier regions as defined by the Randolph Glacier Inventory (RGI Consortium, 2017). Zemp et al. (2019) is based on extrapolation of geodetic and glaciological observations, while Wouters et al. (2019) use gravimetric measurements from the Gravity Recovery and Climate Experiment (GRACE). For some regions, additional estimates are available mostly based on remote sensing data (Table 2.A.1).

These estimates were used to derive an average mass change rate for the period 2006-2015 for each glacier region covered in both Chapter 2 and 3. Where several estimates were available for this period or similar periods, these were averaged and uncertainties obtained from standard error propagation assuming the estimates to be independent. The GRACE estimates were only considered in regions with extensive ice cover due to generally large uncertainties in regions with little ice cover (Wouters et al., 2019). The estimates for the polar regions by Box et al. (2018) were not used since they are based on an earlier version of the data by Wouters et al. (2019).

Individual regional estimates for overlapping periods between 2000 and 2017 were recalculated to represent the period 20062015, prior to averaging with other existing estimates. For Western Canada and USA the mass change rate by Menounos et al. (2019) for 2000-2009 was assumed to hold for 2006-2009, and the rate of $-12 \pm 5 \mathrm{Gt} \mathrm{yr}^{-1}$ for 2009-2018 was assumed to be valid for 20102015. For Iceland the mass change rate by Björnsson et al. (2013) for 2003-2010 was assumed to hold for 2006-2010, and the rate by Foresta et al. (2016) for 2011-2015 was used for the remaining years. The estimate for Iceland by Nilsson et al. (2015) for the period 2003-2009 is similar to the estimate by Björnsson et al. (2013), but was not used since it is based on spatially relatively scarce remote sensing data compared to Björnsson et al. (2013), which is based on detailed glaciological and geodetic balances. The GRACE estimate for Iceland was not used since it deviates strongly from the estimate by Zemp et al. (2019) which is well-constrained by direct observations in this region, while the GRACE estimate may have been affected by the mass change signal from ice masses in southeast Greenland and processes in the Earth mantle cause by isostatic adjustments since the end of the 19th century (Sørensen et al., 2017). For the Low Latitudes ( $>99 \%$ of glacier area in the Andes) available mass loss estimates differ considerably. Zemp et al. (2019)'s high estimate relies on extrapolation of observations from less than $1 \%$ of the glacier area, while the low estimate by Braun et al. (2019) for the Andes may underestimate mass loss due to incomplete coverage and systematic errors in their derived digital elevation models due to radar penetration. In the absence of other estimates for this period the average of both estimates is used. For Arctic Canada and the Southern Andes, the estimates by Zemp et al. (2019) were not considered since they rely on observations from less than $5 \%$ of the glacier area. The regional estimates by Gardner et al. (2013) for the period 2003-2009 informed AR5 and are given for comparison but not included in the composite estimate for 2006-2015.

Table 2A.1 | Regional estimates of glacier mass budget in three different units. Only estimates from the studies marked in bold were used to derive the average SROCC estimates. Regional glacier area $A$ and volume $V$ are taken from the Randolph Glacier Inventory (RGI Consortium, 2017) and Farinotti et al. (2019), respectively. Method geod. refers to the geodetic method (using elevation changes) and gl. refers to the glaciological method (based on in situ mass-balance observations). Results are given for various aggregated areas including among others all regions combined (global), and global excluding the Antarctic (A) and Greenland (G) periphery. All regional estimates (in $\mathrm{kg} \mathrm{m}^{-2} \mathrm{yr}^{-1}$ ) are shown in Figures 2.4 and 3.8). SLE is sea level equivalent.

\begin{tabular}{|c|c|c|c|c|c|c|}
\hline Mass budget & & $\mathrm{kg} \mathrm{m}^{-2} \mathrm{yr}^{-1}$ & Gt $\mathrm{yr}^{-1}$ & mm SLE $y r^{-1}$ & Reference & Method \\
\hline \multirow{7}{*}{$\begin{array}{l}\text { Alaska, } \\
A=86,725 \mathrm{~km}^{2} \\
V=43.3 \pm 11.2 \mathrm{~mm} \text { SLE }\end{array}$} & 2003-2009 & $-570 \pm 200$ & $-50 \pm 17$ & $0.14 \pm 0.05$ & Gardner et al. (2013) & GRACE \\
\hline & $1986-2005$ & $-610 \pm 280$ & $-53 \pm 24$ & $0.15 \pm 0.07$ & Box et al. (2018) & GRACE, gl. \\
\hline & 1994-2013 & $-865 \pm 130$ & $-75 \pm 11$ & $0.21 \pm 0.03$ & Larsen et al. (2015) & geod. \\
\hline & $2006-2015$ & $-710 \pm 340$ & $-61 \pm 30$ & $0.17 \pm 0.08$ & Box et al. (2018) & GRACE, gl. \\
\hline & $2006-2015$ & $-570 \pm 180$ & $-49 \pm 16$ & $0.14 \pm 0.04$ & Wouters et al. (2019) & GRACE \\
\hline & 2006-2015 & $-830 \pm 190$ & $-71 \pm 17$ & $0.20 \pm 0.05$ & Zemp et al. (2019) & gl., geod. \\
\hline & 2006-2015 & $-700 \pm 180$ & $-60 \pm 16$ & $0.17 \pm 0.04$ & SROCC & \\
\hline \multirow{6}{*}{$\begin{array}{l}\text { Western Canada and USA, } \\
A=14,524 \mathrm{~km}^{2} \text {, } \\
h V=2.6 \pm 0.7 \mathrm{~mm} \text { SLE }\end{array}$} & 2003-2009 & $-930 \pm 230$ & $-14 \pm 3$ & $0.04 \pm 0.01$ & Gardner et al. (2013) & gl. \\
\hline & $2000-2009$ & $-200 \pm 250$ & $-3 \pm 3$ & $0.01 \pm 0.01$ & Menounos et al. (2019) & geod. \\
\hline & 2009-2018 & $-860 \pm 320$ & $-12 \pm 5$ & $0.03 \pm 0.01$ & Menounos et al. (2019) & geod. \\
\hline & 2006-2015 & $-410 \pm 1,500$ & $-6 \pm 22$ & $0.02 \pm 0.06$ & Wouters et al. (2019) & GRACE \\
\hline & $2006-2015$ & $-800 \pm 400$ & $-11 \pm 6$ & $0.03 \pm 0.02$ & Zemp et al. (2019) & gl., geod. \\
\hline & 2006-2015 & $-500 \pm 910$ & $-8 \pm 13$ & $0.02 \pm 0.04$ & SROCC & \\
\hline
\end{tabular}


Mass budget

$\mathrm{kg} \mathrm{m}^{-2} \mathrm{yr}^{-1}$

Gt $y r^{-1}$

$\mathrm{mm} \mathrm{SLE} \mathrm{yr}^{-1}$

Reference

Method

Iceland,

$A=11,060 \mathrm{~km}^{2}$,

$\mathrm{V}=9.1 \pm 2.4 \mathrm{~mm} \mathrm{SLE}{ }^{*}$

Scandinavia,

$\mathrm{A}=2,949 \mathrm{~km}^{2}$

$\mathrm{V}=0.7 \pm 0.2 \mathrm{~mm} \mathrm{SLE}$

\begin{tabular}{|c|c|c|c|c|c|}
\hline 2003-2009 & $-910 \pm 150$ & $-10 \pm 2$ & $0.03 \pm 0.01$ & Gardner et al. (2013) & GRACE, gl. \\
\hline 1986-2005 & $-360 \pm 630$ & $-4 \pm 7$ & $0.01 \pm 0.02$ & Box et al. (2018) & GRACE, gl. \\
\hline 1995-2010 & $-860 \pm 140$ & $-10 \pm 2$ & $0.03 \pm 0.00$ & Björnsson et al. (2013) & gl. geod. \\
\hline 2003-2010 & $-995 \pm 140$ & $-11 \pm 2$ & $0.03 \pm 0.00$ & Björnsson et al. (2013) & gl. geod. \\
\hline 2003-2009 & $-890 \pm 250$ & $-10 \pm 3$ & $0.03 \pm 0.01$ & Nilsson et al. (2015) & geod. \\
\hline 2011-2015 & $-590 \pm 70$ & $-6 \pm 1$ & $0.02 \pm 0.00$ & Foresta et al. (2016) & geod. \\
\hline $2006-2015$ & $-910 \pm 190$ & $-10 \pm 2$ & $0.03 \pm 0.01$ & Wouters et al. (2019) & GRACE \\
\hline $2006-2015$ & $-620 \pm 410$ & $-7 \pm 4$ & $0.02 \pm 0.01$ & Zemp et al. (2019) & gl., geod. \\
\hline 2006-2015 & $-690 \pm 260$ & $-7 \pm 3$ & $0.02 \pm 0.01$ & SROCC & \\
\hline 2003-2009 & $-610 \pm 140$ & $-2 \pm 0$ & $0.01 \pm 0.00$ & Gardner et al. (2013) & gl. \\
\hline $1986-2005$ & $-120 \pm 1,170$ & $-0 \pm 3$ & $0.00 \pm 0.01$ & Box et al. (2018) & GRACE, gl. \\
\hline 2006-2015 & $230 \pm 3,820$ & $1 \pm 11$ & $-0.00 \pm 0.03$ & Wouters et al. (2019) & GRACE \\
\hline $2006-2015$ & $-660 \pm 270$ & $-2 \pm 1$ & $0.01 \pm 0.00$ & Zemp et al. (2019) & gl., geod \\
\hline 2006-2015 & $-370 \pm 1,220$ & $-1 \pm 4$ & $0.00 \pm 0.01$ & Box et al. (2018) & GRACE, gl. \\
\hline 2006-2015 & $-660 \pm 270$ & $-2 \pm 1$ & $0.01 \pm 0.00$ & SROCC & \\
\hline 2003-2009 & $-630 \pm 310$ & $-2 \pm 0$ & $0.01 \pm 0.00$ & Gardner et al. (2013) & gl. \\
\hline $2006-2015$ & $890 \pm 1,850$ & $2 \pm 5$ & $-0.01 \pm 0.01$ & Wouters et al. (2019) & GRACE \\
\hline 2006-2015 & $-400 \pm 310$ & $-1 \pm 1$ & $0.00 \pm 0.00$ & Zemp et al. (2019) & gl., geod. \\
\hline 2006-2015 & $-400 \pm 310$ & $-1 \pm 1$ & $0.00 \pm 0.00$ & SROCC & \\
\hline 2003-2009 & $-1,060 \pm 170$ & $-2 \pm 0$ & $0.01 \pm 0.00$ & Gardner et al. (2013) & gl. \\
\hline $2006-2015$ & $100 \pm 510$ & $0 \pm 1$ & $-0.00 \pm 0.00$ & Wouters et al. (2019) & GRACE \\
\hline 2006-2015 & $-910 \pm 70$ & $-2 \pm 0$ & $0.01 \pm 0.00$ & Zemp et al. (2019) & gl., geod. \\
\hline 2006-2015 & $-910 \pm 70$ & $-2 \pm 0$ & $0.01 \pm 0.00$ & SROCC & \\
\hline 2003-2009 & $-900 \pm 160$ & $-1 \pm 0$ & $0.00 \pm 0.00$ & Gardner et al. (2013) & gl. \\
\hline 2006-2015 & $-650 \pm 3000$ & $-1 \pm 4$ & $0.00 \pm 0.01$ & Wouters et al. (2019) & GRACE \\
\hline 2006-2015 & $-880 \pm 570$ & $-1 \pm 1$ & $0.00 \pm 0.00$ & Zemp et al. (2019) & gl., geod. \\
\hline 2006-2015 & $-880 \pm 570$ & $-1 \pm 1$ & $0.00 \pm 0.00$ & SROCC & \\
\hline 2003-2009 & $-220 \pm 100$ & $-26 \pm 12$ & $-0.07 \pm 0.03$ & Gardner et al. (2013) & GRACE, geod. \\
\hline 2006-2015 & $-110 \pm 140$ & $-11 \pm 14$ & $0.03 \pm 0.04$ & Wouters et al. (2019) & GRACE \\
\hline 2006-2015 & $-190 \pm 70$ & $-18 \pm 7$ & $0.05 \pm 0.02$ & Zemp et al. (2019) & gl., geod. \\
\hline 2000-2016 & $-180 \pm 40$ & $-16 \pm 4$ & $0.04 \pm 0.01$ & Brun et al. (2017) & geod. \\
\hline 2006-2015 & $-150 \pm 110$ & $-14 \pm 11$ & $0.04 \pm 0.03$ & SROCC & \\
\hline 2003-2009 & $-1,080 \pm 360$ & $-4 \pm 1$ & $0.01 \pm 0.00$ & Gardner et al. (2013) & gl. \\
\hline 2000-2013 & $-230 \pm 40$ & $-1 \pm 0$ & $0.00 \pm 0.00$ & Braun et al. (2019) & geod. \\
\hline 2006-2015 & $1,560 \pm 510$ & $4 \pm 1$ & $-0.01 \pm 0.00$ & Wouters et al. (2019) & GRACE \\
\hline 2006-2015 & $-940 \pm 820$ & $-2 \pm 2$ & $0.01 \pm 0.00$ & Zemp et al. (2019) & gl., geod \\
\hline 2006-2015 & $-590 \pm 580$ & $-1 \pm 1$ & $0.00 \pm 0.00$ & SROCC & \\
\hline 2003-2009 & $-990 \pm 360$ & $-29 \pm 10$ & $0.08 \pm 0.03$ & Gardner et al. (2013) & GRACE \\
\hline 2006-2015 & $-1,070 \pm 240$ & $-31 \pm 7$ & $0.09 \pm 0.02$ & Wouters et al. (2019) & GRACE \\
\hline 2006-2015 & $-1300 \pm 380$ & $-35 \pm 11$ & $0.10 \pm 0.03$ & Zemp et al. (2019) & gl., geod \\
\hline 2000-2015 & $-640 \pm 20$ & $-19 \pm 1$ & $0.05 \pm 0.00$ & Braun et al. (2019) & geod. \\
\hline 2011-2017 & $-1,280 \pm 120$ & $-21 \pm 2$ & $0.06 \pm 0.01$ & Foresta et al. $(2018)^{* *}$ & geod. \\
\hline 2006-2015 & $-860 \pm 170$ & $-25 \pm 4$ & $0.07 \pm 0.01$ & SROCC & \\
\hline
\end{tabular}

Low Latitudes,

$\mathrm{A}=23,41 \mathrm{~km}^{2}$,

$\mathrm{V}=0.2 \pm 0.1 \mathrm{~mm} \mathrm{SLE}$

Southern Andes,

$\mathrm{A}=29,429 \mathrm{~km}^{2}$

$\mathrm{V}=12.8 \pm 3.3 \mathrm{~mm}$ SLE

$A=2,410 \mathrm{~km}$

$\mathrm{V}=0.3+0.1 \mathrm{~mm} \mathrm{SLE}$

Central Europe

$=2,092 \mathrm{~km}^{2}$

$A=1,307 \mathrm{~km}^{2}$

$\mathrm{V}=0.2 \pm 0.0 \mathrm{~mm} \mathrm{SLE}$

$A=97,605 \mathrm{~km}^{2}$.

$\mathrm{V}=16.9 \pm 2.7 \mathrm{~mm} \mathrm{SLE}$ 


\begin{tabular}{|c|c|c|c|c|c|c|}
\hline Mass budget & & $\mathrm{kg} \mathrm{m}^{-2} \mathrm{yr}^{-1}$ & Gt yr $\mathbf{r}^{-1}$ & mm SLE yr ${ }^{-1}$ & Reference & Method \\
\hline \multirow{4}{*}{$\begin{array}{l}\text { New Zealand, } \\
A=1,162 \mathrm{~km}^{2}, \\
V=0.2 \pm 0.0 \mathrm{~mm} \text { SLE }\end{array}$} & 2003-2009 & $-320 \pm 780$ & $0 \pm 1$ & $0.00 \pm 0.00$ & Gardner et al. (2013) & gl. \\
\hline & $2006-2015$ & $110 \pm 780$ & $0 \pm 1$ & $0.00 \pm 0.00$ & Wouters et al. (2019) & GRACE \\
\hline & $2006-2015$ & $-590 \pm 1140$ & $-1 \pm 1$ & $0.00 \pm 0.00$ & Zemp et al. (2019) & gl., geod \\
\hline & 2006-2015 & $-590 \pm 1140$ & $-1 \pm 1$ & $0.00 \pm 0.00$ & SROCC & \\
\hline \multirow{11}{*}{$\begin{array}{l}\text { Arctic Canada North, } \\
A=105,111 \mathrm{~km}^{2} \\
\mathrm{~V}=64.8 \pm 16.8 \mathrm{~mm} \text { SLE }\end{array}$} & 2003-2009 & $-310 \pm 40$ & $-33 \pm 4$ & $0.09 \pm 0.01$ & Gardner et al. (2013) & GRACE, geod. \\
\hline & 1958-1995 & $-114 \pm 110$ & $-12 \pm 12$ & $0.03 \pm 0.03$ & Noël et al. (2018) & Model \\
\hline & 1996-2015 & $-270 \pm 110$ & $-28 \pm 12$ & $0.08 \pm 0.03$ & Noël et al. (2018) & Model \\
\hline & $1991-2014$ & $-170 \pm 50$ & $-16 \pm 2$ & $0.04 \pm 0.00$ & Millan et al. (2017) & \multirow{3}{*}{ Model } \\
\hline & $1991-2005$ & $-60 \pm 20$ & $-6 \pm 1$ & $0.02 \pm 0.00$ & Millan et al. (2017) & \\
\hline & 2005-2014 & $-340 \pm 30$ & $-33 \pm 3$ & $0.09 \pm 0.01$ & Millan et al. (2017) & \\
\hline & 2003-2009 & $-260 \pm 60$ & $-50 \pm 9$ & $0.17 \pm 0.02$ & Nilsson et al. (2015) & geod. \\
\hline & 2006-2015 & $-400 \pm 110$ & $-41 \pm 12$ & $0.11 \pm 0.03$ & Noël et al. (2018) & Model \\
\hline & 2006-2015 & $-390 \pm 30$ & $-41 \pm 4$ & $0.12 \pm 0.01$ & Wouters et al. (2019) & GRACE \\
\hline & 2006-2015 & $-540 \pm 800$ & $-56 \pm 84$ & $0.15 \pm 0.23$ & Zemp et al. (2019) & gl., geod. \\
\hline & 2006-2015 & $-380 \pm 80$ & $-39 \pm 8$ & $0.11 \pm 0.02$ & SROCC & \\
\hline \multirow{8}{*}{$\begin{array}{l}\text { Arctic Canada South, } \\
A=40,888 \mathrm{~km}^{2}, \\
V=20.5 \pm 5.3 \mathrm{~mm} \text { SLE }\end{array}$} & 2003-2009 & $-660 \pm 110$ & $-27 \pm 4$ & $0.07 \pm 0.01$ & Gardner et al. (2013) & GRACE, geod. \\
\hline & 1958-1995 & $-280 \pm 100$ & $-12 \pm 5$ & $0.03 \pm 0.01$ & Noël et al. (2018) & Model \\
\hline & 1996-2015 & $-510 \pm 100$ & $-22 \pm 5$ & $0.06 \pm 0.01$ & Noël et al. (2018) & Model \\
\hline & 2003-2009 & $-550 \pm 130$ & $-23 \pm 5$ & $0.06 \pm 0.01$ & Nilsson et al. (2015) & geod. \\
\hline & 2006-2015 & $-650 \pm 100$ & $-28 \pm 5$ & $0.08 \pm 0.01$ & Noël et al. (2018) & Model \\
\hline & 2006-2015 & $-940 \pm 210$ & $-39 \pm 9$ & $0.11 \pm 0.02$ & Wouters et al. (2019) & GRACE \\
\hline & 2006-2015 & $-540 \pm 700$ & $-22 \pm 28$ & $0.06 \pm 0.08$ & Zemp et al. (2019) & gl., geod. \\
\hline & 2006-2015 & $-800 \pm 220$ & $-33 \pm 9$ & $0.09 \pm 0.03$ & SROCC & \\
\hline \multirow{6}{*}{$\begin{array}{l}\text { Greenland periphery, } \\
A=89,717 \mathrm{~km}^{2} \\
V=33.6 \pm 8.7 \mathrm{~mm} \text { SLE }\end{array}$} & 2003-2009 & $-420 \pm 70$ & $-38 \pm 7$ & $0.10 \pm 0.02$ & Gardner et al. (2013) & geod. \\
\hline & 1958-1996 & $-140 \pm 190$ & $-11 \pm 16$ & $0.03 \pm 0.04$ & Noël et al. (2017) & Model \\
\hline & 1997-2015 & $-400 \pm 180$ & $-36 \pm 16$ & $0.10 \pm 0.04$ & Noël et al. (2017) & Model \\
\hline & 2006-2015 & $-510 \pm 190$ & $-42 \pm 16$ & $0.11 \pm 0.04$ & Noël et al. (2017) & Model \\
\hline & $2006-2015$ & $-635 \pm 200$ & $-53 \pm 17$ & $0.15 \pm 0.05$ & Zemp et al. (2019) & gl., geod. \\
\hline & 2006-2015 & $-570 \pm 200$ & $-47 \pm 16$ & $0.13 \pm 0.04$ & SROCC & \\
\hline \multirow{9}{*}{$\begin{array}{l}\text { Svalbard, } \\
A=33,959 \mathrm{~km}^{2} \\
V=17.3 \pm 4.5 \mathrm{~mm} \mathrm{SLE***}\end{array}$} & 2003-2009 & $-130 \pm 60$ & $-5 \pm 2$ & $0.01 \pm 0.01$ & Gardner et al. (2013) & GRACE, geod. \\
\hline & $1986-2005$ & $-240 \pm 120$ & $-8 \pm 4$ & $0.02 \pm 0.01$ & Box et al. (2018) & GRACE, gl. \\
\hline & $2003-2009$ & $-120 \pm 80$ & $-4 \pm 3$ & $0.01 \pm 0.01$ & Nilsson et al. (2015) & geod. \\
\hline & 2003-2013 & -260 & -9 & 0.02 & Aas et al. (2016) & Model \\
\hline & 2004-2013 & -210 & -7 & 0.02 & Østby et al. (2017) & Model \\
\hline & 2006-2015 & $-250 \pm 160$ & $-8 \pm 5$ & $0.02 \pm 0.02$ & Box et al. (2018) & GRACE, gl. \\
\hline & $2006-2015$ & $-200 \pm 40$ & $-7 \pm 2$ & $0.02 \pm 0.00$ & Wouters et al. (2019) & GRACE \\
\hline & 2006-2015 & $-400 \pm 230$ & $-13 \pm 7$ & $0.04 \pm 0.02$ & Zemp et al. (2019) & gl., geod \\
\hline & 2006-2015 & $-270 \pm 170$ & $-9 \pm 5$ & $0.02 \pm 0.01$ & SROCC & \\
\hline
\end{tabular}




\begin{tabular}{|c|c|c|c|c|c|c|}
\hline Mass budget & & $\mathrm{kg} \mathrm{m}^{-2} \mathrm{yr}^{-1}$ & Gt $\mathrm{yr}^{-1}$ & mm SLE yr ${ }^{-1}$ & Reference & Method \\
\hline \multirow{7}{*}{$\begin{array}{l}\text { Russian Arctic, } \\
A=51,592 \mathrm{~km}^{2}, \\
V=32.0 \pm 8.3 \mathrm{~mm} \text { SLE }\end{array}$} & 2003-2009 & $-210 \pm 80$ & $-11 \pm 4$ & $0.03 \pm 0.01$ & Gardner et al. (2013) & GRACE, geod. \\
\hline & 1986-2005 & $-210 \pm 190$ & $-11 \pm 10$ & $0.03 \pm 0.03$ & Box et al. (2018) & GRACE, gl. \\
\hline & 2003-2009 & $-140 \pm 50$ & $-7 \pm 3$ & $0.02 \pm 0.01$ & Nilsson et al. (2015) & geod. \\
\hline & 2006-2015 & $-200 \pm 250$ & $-11 \pm 13$ & $0.03 \pm 0.04$ & Box et al. (2018) & G., gl. \\
\hline & $2006-2015$ & $-220 \pm 40$ & $-11 \pm 2$ & $0.03 \pm 0.01$ & Wouters et al. (2019) & GRACE \\
\hline & $2006-2015$ & $-400 \pm 370$ & $-20 \pm 16$ & $0.06 \pm 0.04$ & Zemp et al. (2019) & gl., geod \\
\hline & 2006-2015 & $-300 \pm 270$ & $-15 \pm 12$ & $0.04 \pm 0.03$ & SROCC & \\
\hline \multirow{3}{*}{$\begin{array}{l}\text { Antarctic periphery, } \\
A=132,867 \mathrm{~km}^{2} \\
V=69.4 \pm 18 \mathrm{~mm} \text { SLE }\end{array}$} & 2003-2009 & $-50 \pm 70$ & $-6 \pm 10$ & $0.02 \pm 0.03$ & Gardner et al. (2013) & geod. \\
\hline & 2006-2015 & $-90 \pm 860$ & $-11 \pm 108$ & $0.03 \pm 0.3$ & Zemp et al. (2019) & gl., geod \\
\hline & 2006-2015 & $-90 \pm 860$ & $-11 \pm 108$ & $0.03 \pm 0.3$ & SROCC & \\
\hline $\begin{array}{l}11 \text { Mountain regions covered in } \\
\text { Chapter } 2, A=251,604 \mathrm{~km}^{2} \text {, } \\
V=87 \pm 15 \mathrm{~mm} \mathrm{SLE}\end{array}$ & 2006-2015 & $-490 \pm 100$ & $-123 \pm 24$ & $0.34 \pm 0.07$ & SROCC & \\
\hline $\begin{array}{l}\text { Arctic regions**** } \\
\mathrm{A}=422,000 \mathrm{~km}^{2} \\
\mathrm{~V}=221 \pm 25 \mathrm{~mm} \text { SLE }\end{array}$ & 2006-2015 & $-500 \pm 70$ & $-213 \pm 29$ & $-0.59 \pm 0.08$ & SROCC & \\
\hline $\begin{array}{l}\text { Global excl. A+G periphery, } \\
A=483,155 \mathrm{~km}^{2} \\
V=221 \pm 23 \mathrm{~mm} \text { SLE }\end{array}$ & 2006-2015 & $-460 \pm 60$ & $-220 \pm 30$ & $0.61 \pm 0.08$ & SROCC & \\
\hline $\begin{array}{l}\text { Global, } \\
A=705,739 \mathrm{~km}^{2} \\
V=324 \pm 84 \mathrm{~mm} \text { SLE }\end{array}$ & 2006-2015 & $-390 \pm 160$ & $-278 \pm 113$ & $0.77 \pm 0.31$ & SROCC & \\
\hline
\end{tabular}

Notes:

*Björnsson and Pálsson (2008) report a volume of 9 mm SLE based on radio-echo sounding data.

**only Northern and Southern Patagonian Ice Fields (38\% of regional area).

***Fürst et al. (2018) report a volume of $15.3 \pm 2.6 \mathrm{~mm}$ SLE.

****including Alaska, Iceland, and Scandinavia (covered in Chapter 2), and Arctic Canada, Greenland periphery, Russian Arctic and Svalbard (covered in Chapter 3). 